\title{
PRODUCTIVITY AND INJECTIVITY OF HORIZONTAL WELLS
}

Annual Report for the Period

March 10, 1993 to March 9, 1994

\author{
by \\ F. John Fayers \\ Khalid Aziz \\ . Thomas A. Hewett \\ Sepehr Arbabi
}

October 1994

Work Performed Under Contract No. DE-FG22-93BC14862

Prepared for

U.S. Department of Energy

Assistant Secretary for Fossil Energy

Thomas B. Reid, Project Manager

Bartlesville Project Office

P.O. Box 1398

Bartlesville, OK 74005

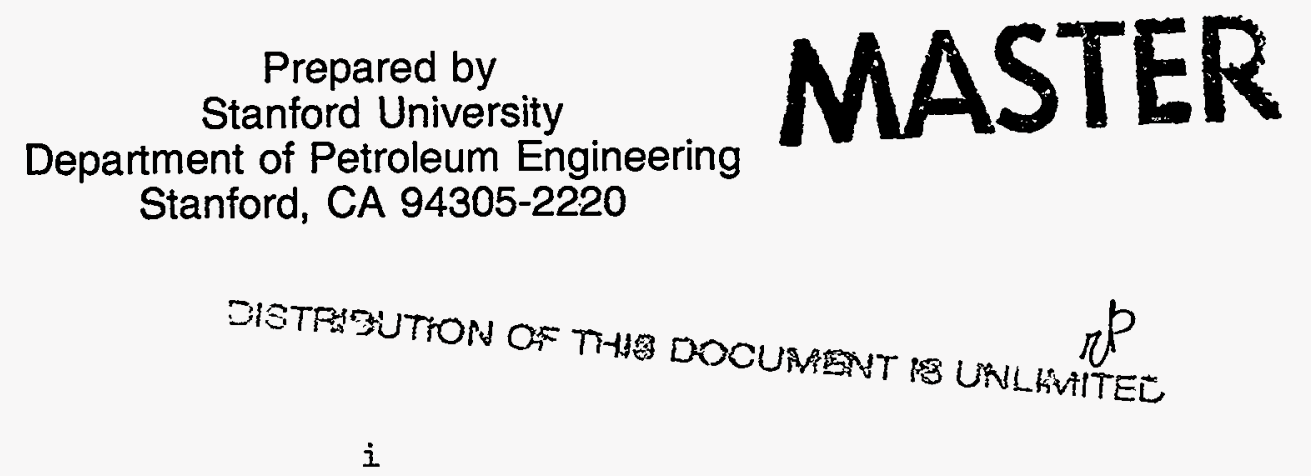


ii 


\section{DISCLAIMER}

This report was prepared as an account of work sponsored by an agency of the United States. Government. Neither the United States Government nor any agency thereof, nor any of their employees, make any warranty, express or implied, or assumes any legal liability or responsibility for the accuracy, completeness, or usefulness of any information, apparatus, product, or process disclosed, or represents that its use would not infringe privately owned rights. Reference herein to any specific commercial product, process, or service by trade name, trademark, manufacturer, or otherwise does not necessarily constitute or imply its endorsement, recommendation, or favoring by the United States Government or any agency thereof. The views and opinions of authors expressed herein do not necessarily state or reflect those of the United States Government or any agency thereof. 


\section{DISCLAIMER}

Portions of this document may be illegible in electronic image products. Images are produced from the best available original document. 


\title{
Management Summary
}

\section{DOE Approval}

The DOE approval for the research grant to the Stanford Project on the Productivity and Injectivity of Horizontal Wells was received in early March 1993. The Project formally commenced on March 10, 1993.

\section{Industrial Affiliates Program}

The DOE Project operates in association with an Industrial Affiliates program on horizontal wells, for which oil company membership has also been initiated at Stanford during 1993. The membership during this year comprised the following organizations:

\author{
AGIP (Italy) \\ ARTEP/IFP (France) \\ $\mathrm{BP}$ \\ Chevron \\ Gas Research Institute \\ Marathon \\ Norsk Hydro (Norway) \\ Petrobras America (Brazil) \\ Texaco
}

\section{Project Goals}

The Project has eight principal goals to be studied and developed over a five year period. These goals are as follows:

TASK 1: Advanced Modeling of Horizontal Wells - Develop special gridding techniques and associated averaging algorithms for accurate simulation of HW-performance.

TASK 2: Investigate and Incorporate the Effects of Reservoir Heterogeneties - Study impacts of various types of heterogeneity and develop methods for incorporating their effects in both fine grid and coarse grid models.

TASK 3: Develop Improved Methods for Calculating Multi-Phase Pressure Drops within the Wellbore - Plan, execute, and interpret two-phase flow experiments at an oil company research facility, and use results to analyze/validate a new two-phase model.

TASK 4: Pseudo-Functions - Define improved methods for computing two-phase pseudofunctions for effective relative permeabilities for coarse grid blocks near an $\mathrm{HW}$ - determine sensitivities to heterogeneities, flow conditions, skin factors, etc.

TASK 5: Develop Multi-Well Models - Develop numerical techniques and software in a parallel computing architecture capable of interactively coupling multiple detailed HWmodels to a large scale reservoir simulator. 
TASK 6: Test HW-Models with Field Examples - Work with affiliate's member companies to establish HW-modeling capabilities from field measurements, particularly for pathological problem cases.

TASK 7: EOR Applications - Provide and implement practical HW aspects into modeling of EOR processes - miscible gas, steam displacement, in-situ combustion.

TASK 8: Application Studies and Their Optimization - Seek field opportunities for HW's and study their best implementation in various reservoir scenarios e.g., multiple laterals, hydraulic fracture variants, etc.

Tasks 1 to 3 were the primary focus of activities scheduled for 1993, and the annual report is therefore written around these three tasks, which are also ongoing in 1994.

\section{Large-Scale Flow Loop Experiments}

The funding from the Industrial Affiliate's Project is mainly expended on a series of large scale experiments in an outdoor flow loop being conducted at the Marathon Research Center in Littleton, Colorado. Contractual arrangements were set with Marathon to undertake these experiments, for which a substantial part of the costs $(\sim 50 \%)$ is borne by Marathon. Considerable modification of the $100 \mathrm{ft}$ well model has been required, where new instrumentation has also been added to the transparent acrylic pipe sections (6.2 inch diameter) and the feeds to the perforations. Arrangements to allow injection of air or nitrogen have been added to the manifolds of the perforation feeder lines. Single phase baseline experiments with both water and oil have been completed with measurement of very small pressure drops using liquid manometer gauges. New Rosemount DP-instruments have been purchased for the larger two-phase measurements and some have been installed. Two-phase flow experiments for air/water and nitrogen/oil fluids have also been run. Due to limitations on separator capacity high flow rate gas/liquid experiments have not yet been possible, and this requirement is under review for 1994. A special two-phase liquid holdup meter based on a capacitance measurement technique has been designed and shown to be capable of measuring the pulsating variations of liquid fraction during slug flow. Initial interpretation of the two-phase measurements suggests pressure drops are larger than those predicted by standard smooth pipe correlations in the literature.

\section{Coning and Cresting Predictions}

Methods of prediction for coning and cresting in horizontal and vertical wells have been examined. Many of the analytic equations for critical rates for both.HWs and VWs are found to be in error by comparison with accurate simulation results for homogeneous reservoirs. The error for HWs can be particularly serious. An important property for HWs with wide spacing is not so much their critical cresting rate (which is a rather diffuse limit), but the very long times for breakthrough of a gas or water crest. Simple analytic expressions for gas or water breakthrough times have been investigated for HWs and shown to be reliable at wider spacings. An analytic theory due to Wheatley has been demonstrated to be accurate for critical rates in partially completed VWs, but no equivalent analytical treatment is available for the poorly resolved critical cresting rate of an HW. 


\section{Effects of Heterogeneities on Horizontal Well Performance}

Work on the important impacts of heterogeneities on performance of HWs was started. A Masters Thesis on one aspect of this project has been completed. In this work by Tomomi Yamada (a Ph.D. student with Prof. Thomas Hewett) flow simulations have been used to investigate the variability of production behavior for a $\mathrm{HW}$ in a reservoir containing stochastic distributions of impermeable shales. To construct a probability distribution of oil recovery, a large number of stochastic realizations were run in the flow simulations. Setting up the necessary simulation runs requires extended and careful effort. It was found that the ranking of oil recovery can be realized by a single parameter, thus in future for this type of problem the distribution of oil recovery can be constructed without performing massive flow calculations. The flow parameter is the production-based effective permeability which is introduced in the thesis. Its application is demonstrated for a water flooding problem with horizontal injectors and producers.

\section{Variation of Productivity Index Along an HW of Finite Length}

Work was undertaken by M.S. student Obaid Al-Harthy in association with the SUPRI-D Project under Prof. Roland Horne (Well Testing Methods) to evaluate the local variations in productivity index associated with HWs of finite length. The flow becomes spherical near the edges of the well, rather than cylindrical in the central sections, giving rise to substantial increases in productivity $(\sim 23 \%)$ in the regions close to the ends. A new analytical model for computing productivities was developed based on Green's functions, and then interpreted for use in evaluating the completion factor required to join the well locally to its Cartesian grid blocks for a finite difference model. In the central region, the effective radius for the well block average pressure was found to be close to the standard Peaceman formula for an infinite well as used in conventional black oil simulators. Position varying values can be evaluated from the new model for well blocks near the ends, including handling the case where the completed well interval at the end only partially penetrates its well block. This work will be reported in detail in a M.S. Thesis nearing completion.

\section{Generalized Gridding Methods}

Development has started on 3D-generalized Voronoi methods for horizontal wells. This work is underway by Santosh Verma, a Ph.D. student under Prof. Khalid Aziz. In the first stage of this development a 2D-Voronoi grid is used in a plane perpendicular to the horizontal well with a non-uniform Cartesian mesh in the horizontal plane. The Voronoi grid can for example be a hexagonal grid close to the well to describe better the nearly radial flow in this region. In other places in the reservoir, 2D Voronoi grids can be chosen in the horizontal plane to represent vertical wells. A large computer code is being developed using the $\mathrm{C}++$ language.

\section{Improved Predictive Modeling of Two-Phase Flow within the Wellbore}

Work has started on improved modeling of two-phase flow pressure drops in HWs. This work is based on a mechanistic approach which involves as the first step the identification of the flow regimes, which vary considerably with the sign of the angle of inclination. Subsequently, 
the pressure drops or liquid holdups can be calculated. An improved methodology for this treatment is being developed by Nick Petalas, a professional programmer in the P.E. Department in association with Prof. Khalid Aziz. This work is also supported by the Stanford Reservoir Simulation Industrial Affliates Program (SUPRI-B).

\section{Affiliates Progress Review Meeting}

An annual progress review meețing for member companies in the Stanford Horizontal Well Project was held at Stanford on Qctober 7th and 8th. This meeting was well attended and member companies made presentations on their horizontal well interests and technical activities during the second day. Useful input to future project goals was received from the member organizations. The next meeting is scheduled for September 22nd and 23rd, to be adjacent to the SPE Fall Meeting for the benefit of travel arrangements for overseas members.

\section{Completion of Review Report}

A review report entitled "Opportunities for Horizontal Wells and Problems in Predicting Their Performance" was written with Dr. Arbabi and Profs. Fayers and Aziz as authors. It covers major aspects of current status of prediction methods for the performance of HWs. The report points out uncertainties in the current prediction methods and introduces some possible future topics. This report was released to the affliate members in December 1993, and significant parts are included in this DOE annual review report.

\section{Presentations at Conferences etc.}

A number of presentations were made related to the Project. These include presentations at the Reservoir Recovery Forum, Denver, in April, DOE Peer Review Meeting, Fountain head, Oklahoma, in July, at the Drilling and Completions Forum (Maurer Engineering), Houston, in September, JNOC Symposium on Horizontal Wells, in Japan in November, and at a Petronas/SPE Sponsored Meeting in Malaysia in December. 


\section{Preface: Objectives for 1993}

Stanford Project on Productivity and Injectivity of Horizontal Wells has been set up for the purpose of developing advanced performance prediction methods for horizontal wells in heterogeneous reservoirs. The stimulus for this project arises from the fact that $\mathrm{HWs}$ are now being drilled in large numbers (e.g., over $4200 \mathrm{HWs}$ drilled in the U.S. to the end of 1993) and their benefits have provided the one example of an advanced oil recovery technique which is proving to be a general economic attraction.

There are eight major objectives, of which in year one the following three tasks were concentrated on:

Task 1: Modeling Horizontal Wells-Establish detailed 3D methods of calculation which will successfully predict horizontal well performance under a range of reservoir and flow conditions. Review both commercial simulators and simple inflow performance relationships used by the industry. Investigate the sensitivity of various parameters on the performance of horizontal wells. Develop modeling techniques and computer codes based on generalized 3D-flexible gridding techniques which can be incorporated into reservoir simulators.

Task 2: Reservoir Characterization-Investigate reservoir heterogeneity descriptions of interest to applications of horizontal wells. Develop averaging techniques in $3 \mathrm{D}$ which will adequately compute effective single phase and two-phase directional permeabilities within the variable gridding characteristics of the model developed in Task 1 . Perform sensitivity studies of the averaging technique to uncertainities in the heterogeneity distribution.

Task 3: Experimental Planning and Interpretation-Critically review technical literature on two-phase flow in pipes and the correlation of these results in terms of their relevance to horizontal wells. Perform sensitivity studies to choose parameter spaces of interest for some typical field conditions, using oil companies' advice. Plan key experiments to investigate sensitivity to parameter variation including inflow distribution, completion variations, void fractions, etc. Perform data analysis including flow pattern distribution, scaling, dependence on perforation intervals, confidence levels, etc. Revise two-phase flow correlations where necessary to produce new methods of calculation for two-phase pressure drop in horizontal wells. Incorporate this capability in Task 1 above.

We report our progress on the above tasks in this Annual Report. The report is organized into chapters, each containing introduction, results and their discussions. 
viii 


\section{Contents}

1 Introduction $\quad 1$

2 Advantageous Reservoir Opportunities for Horizontal Wells 3

2.1 Low Permeability Reservoirs . . . . . . . . . . . . . . . . . . 3

2.2 Reservoirs Sensitive to Gas or Water Coning . . . . . . . . . . . . . . 3

2.3 Heterogeneous Reservoirs ..................... 4

2.4 Faulted and Fractured Reservoirs .................. 5

2.5 Potential Miscible Gas Applications . . . . . . . . . . . . . . . . . . 6

2.6 Thermal Recovery of Heavy Oil .................. 8

3 Factors Influencing the Computation of Well Indices (Review: Tasks 1, 2 and 3)

3.1 Regular Cartesian Grids ... . . . . . . . . . . . . . . . . . 11

3.2 More Advanced Gridding Concepts . . . . . . . . . . . . . . . . . . 13

3.3 Factors Influencing Well Productivity . . . . . . . . . . . . . . . 20

3.4 General Permeability Anisotropy . . . . . . . . . . . . . . . . . 26

3.5 Averaging Procedures for Heterogeneities . . . . . . . . . . . . . 27

4 End Effects On Productivity Index Of Horizontal Wells (Task 1) 32

4.1 Introduction . . . . . . . . . . . . . . . . 32

4.2 Mathematical Expression for PI . . . . . . . . . . . . . . . . 32

4.2.1 Variation of PI Along the Well . . . . . . . . . . . . . . 33

4.2.2 Well PI for Use in Reservoir Simulators . . . . . . . . . . . . . 33

4.3 Comparison with Simulator Results . . . . . . . . . . . . . 34

5 Flexible Voronoi Grids for Reservoir Simulation (Task 1) 42

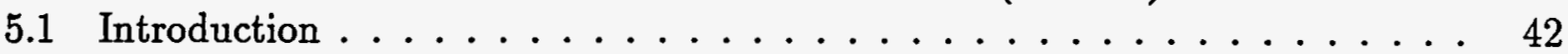

5.2 Mathematical Model ..................... 45

5.3 Handling Anisotropy . . . . . . . . . . . . . . . . . . . . 46

5.4 Generation of Voronoi Grids . . . . . . . . . . . . . . . . . . . . . . . . . . . . . .

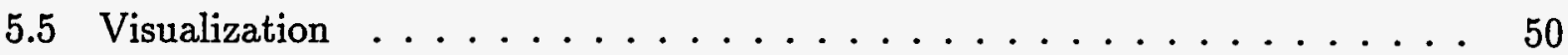

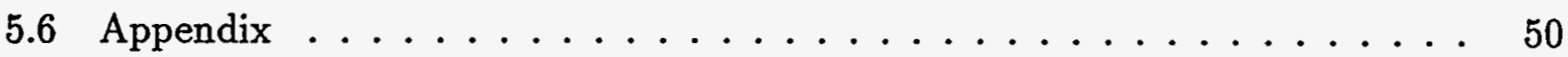

6 Production-Based Effective Vertical Permeability for a Horizontal Well in the Presence of a Stochastic Distribution of Shales (Task 2) 53

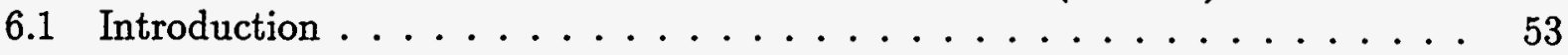


6.2 Model Description . . . . . . . . . . . . . . . . . 54

6.3 Method to Generate Shale Distributions . . . . . . . . . . . . 55

6.4 Variability of Production Performance . . . . . . . . . . . . 55

6.5 Production-Based Effective Vertical Permeability (Kp_eff) . . . . . . . . 60

6.6 Renormalization of the System . . . . . . . . . . . . . . . 65

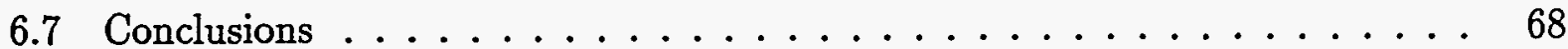

7 Aṇalytic Solutions fọ Coning and Cresting Behavior (Review: Tasks 1 and 3) 70

7.1 Critical Rates . . . . . . . . . . . . . . . . . 70

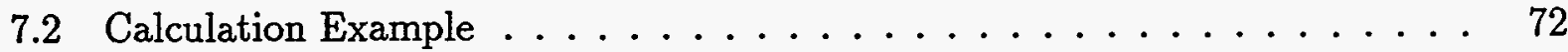

8 Critical Rates and Breakthrough Times in Vertical and Horizontal Wells - Simulation Results (Tasks 1 and 3) 74

8.1 Introduction . . . . . . . . . . . . . . . . . 74

8.2 Summary of Wheatley's Method . . . . . . . . . . . . . . 74

8.3 Critical Rates . . . . . . . . . . . . . . . . . . . . 77

8.4 Breakthrough Times . . . . . . . . . . . . . . . 79

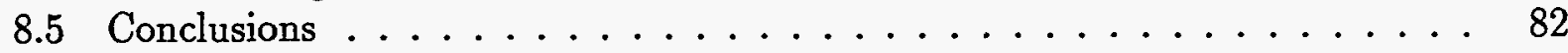

9 Uncertainties Caused by Pressure Drop in the Well (Review: Task 3) 83

9.1 Single-Phase Flow . . . . . . . . . . . . . . . 83

9.2 Two-Phase Flow Behavior . . . . . . . . . . . . . . . . . 84

10 Example Calculations for 3D-Gas Cresting Problem (Tasks 1 and 3) 89

11 Development of a Mechanistic Model for Multiphase Flow in Horizontal Wells (Tasks 1 and 3) 107

11.1 Introduction . . . . . . . . . . . . . . . . . 107

11.2 Model Overview . . . . . . . . . . . . . . . . . . . . 107

11.3 Results . . . . . . . . . . . . . . . . . . . . . . . 109

11.4 Future Development . . . . . . . . . . . . . . . . . . . 109

12 Experiments at Marathon Oil Company (Task 3) 116

12.1 Completed Experiments and Data Analyses . . . . . . . . . . . 116

12.2 Single-Phase Flow Experiments . . . . . . . . . . . . . . . 117

12.3 Two-Phase Flow Experiments . . . . . . . . . . . . . . . 118

12.4 Remarks and Future Plans . . . . . . . . . . . . . . . 122

$\begin{array}{ll}\text { Nomenclature } & 128\end{array}$

$\begin{array}{ll}\text { References } & 130\end{array}$ 


\section{List of Figures}

2.1 Stepped Horizontal Well Used to Produce Three Non-Communicating Reservoir Layers . . . . . . . . . . . . . . . . . . . . . 4 4

2.2 Advantageous Orientation of Horizontal Well to Ensure Contact with Productive Zones in Tight Formations . . . . . . . . . . . . . . 5

2.3 Optimum Placement of Horizontal/Slant Well to Avoid Breakthrough from Gas/Oil and Water/Oil Contacts . . . . . . . . . . . . . . . . 6

2.4 Improved Areal Sweep for Braided Stream Enviornment with Horizontal Wells 7

2.5 Considerations of Effects of Faults or Fractures on Performance of Horizontal Wells . . . . . . . . . . . . . . . . . . . . 8

2.6 Horizontal Well with Transverse and Parallel Multiple Hydraulic Fractures . 9

2.7 Advantages of Horizontal Wells as Injectors in Misicible Gas Applications . . 10

3.1 Special Gridding Schemes . . . . . . . . . . . . . . . . 13

3.2 Generalized Voronoi Grid for Represenatation of Horizontal and Vertical Wells 14

3.3 Correction Factor $\left(f_{c}\right)$ for "Cylindrical" Modules . . . . . . . . . . . 17

3.4 Hybrid-Cartesian Voronoi Grids for Example Problem . . . . . . . . . . . . . 18

3.5 Numerical and Analytical Results for Example Problem . . . . . . . . . . . 19

3.6 3D-Hexagonal Grid and its Equivallencing for Calculating $r_{o} \ldots \ldots \ldots 21$

3.7 Variations of PI for the Example Problem . . . . . . . . . . . . . 23

3.8 Variations of PI as a Function of Open Well Fraction . . . . . . . . . 25

3.9 Averaging Problems Associated with Introduction of Heterogeneities into a Generalized Grid . . . . . . . . . . . . . . . . 28

3.10 Permeability Averaging Area for Connection Between Well Block $i$ and its

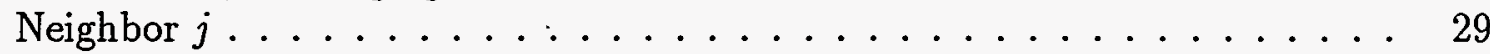

3.11 Parallel and Diagonal Voronoi Grids A and B . . . . . . . . . . 30

3.12 Water Cut History for Voronoi Grids A and B . . . . . . . . . . 31

4.1 Variation of $P I_{D}$ with $L_{D}$ and $t_{D} \ldots \ldots \ldots \ldots \ldots \ldots \ldots \ldots \ldots$

4.2 Variation of $P I_{D}$ with $L_{D}$ and $t_{D} \ldots \ldots \ldots \ldots \ldots \ldots \ldots \ldots$

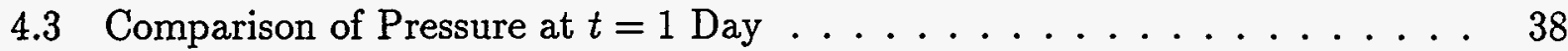

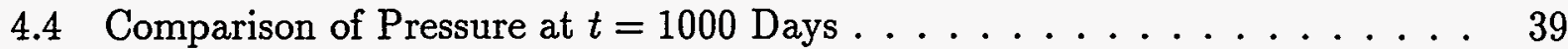

4.5 Comparison of Productivity Index . . . . . . . . . . . . . . . 40

4.6 Comparison of Normalized Productivity Index . . . . . . . . . . . . . 41

5.1 Cartesian Local Grid Refinement . . . . . . . . . . . . . . . . . 43

5.2 Hybrid Local Grid Refinement . . . . . . . . . . . . . . . . . . . . 43

5.3 A Horizontal Well Through a Few Tilted Layers . . . . . . . . . . . . . . . 44 
5.4 Example of Use of 2-D Voronoi Grids Representing Horizontal and Vertical Wells . . . . . . . . . . . . . . . . . . . . 45

5.5 Gradient and Flux in an Anisotropic System . . . . . . . . . . . . . 47

5.6 Intersecting Faces of Voronoi Blocks on Three Sides of a Basic Tetrahedron . 49

6.1 Reservoir Model . . . . . . . . . . . . . . . . . . . . 56

6.2 Stochastic Shale Distributions . . . . . . . . . . . . . . . 57

6.3 Oil Rate, Water Cut Versus Time . . . . . . . . . . . . . . . 58

6.4 Effects of Shale Density and Correlation Range . . . . . . . . . . 59

6.5 Variations of Kv_eff with Shale Density . . . . . . . . . . . . . . . 61

6.6 Oil Recovery Versus Kv_eff . . . . . . . . . . . . . . . . . . 62

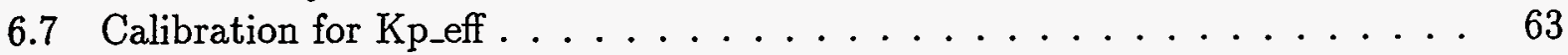

6.8 Oil Recovery Versus Kp_eff . . . . . . . . . . . . . . . . . . . . . . 64

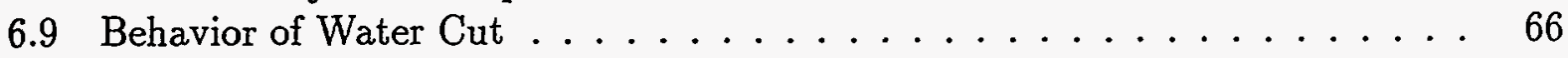

6.10 Distribution of Coarse Grid Kv . . . . . . . . . . . . . 67

6.11 Effect of Boundary Conditions on Flow Resistivity . . . . . . . . . . . . 69

8.1 Schematic of the Water Coning Problem . . . . . . . . . . . . . 75

8.2 Comparison of Critical Rates between Simulation and Analytical Solutions for the Vertical well at Various Completion Penetration Fractions . . . . . .

8.3 Comparison of Critical Rates of Horizontal and Vertical Wells at Various Dimensionless Depths from Simulations . . . . . . . . . . . . . . .

8.4 Variation of the Critical Rate with the Horizontal Dimension of the Reservoir for the Horizontal Well . . . . . . . . . . . . . . . . 80

8.5 Comparison of Breakthrough Times between Simulation and Semianalytical Solutions for Horizontal Wells . . . . . . . . . . . . . . . . .

8.6 Comparison of Breakthrough times for Horizontal and Vertical Wells at Various Supercritical Times for Horizontal and Vertical Wells . . . . . . . . . .

9.1 Effect of Relative Surface Roughness on the Productivity of the Well (Field Example) . . . . . . . . . . . . . . . . . . 84

9.2 Effect of Wellbore Pressure Drop on Breakthrough of a Gas/Oil Contact . . 87

9.3 Prediction of Flow Patterns from a General Mechanistic Model for a Horizon-

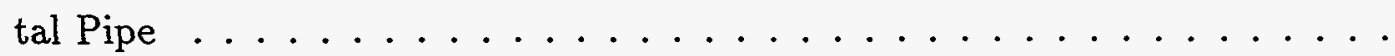

9.4 Prediction of Flow Patterns for a Horizontal Pipe from a Modification of the

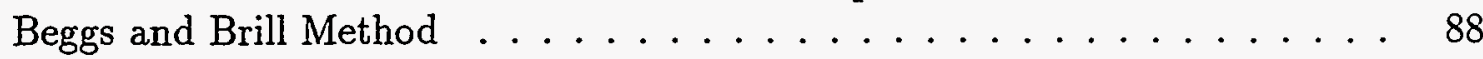

10.1 Reservoir Characteristics and Non-Uniform Gridding in the y-z Plane for the Homogeneous Case . . . . . . . . . . . . . . . . . 89

10.2 Gas Saturation Profiles for the 1D-Problem . . . . . . . . . . . . . 92

10.3 Production Data of Well (Dia=6.18in) with Reservoir Permeability of $500 \mathrm{mD} 93$

10.4 Parameters Along Horizontal Well (Dia $=6.18$ in) for Reservoir Permeability of $500 \mathrm{mD} \ldots \ldots \ldots \ldots \ldots \ldots$. . . . . . . . . . . . 94

10.5 Production Data of Well (Dia=4.28in) with Reservoir Permeability of $100 \mathrm{mD} 95$

10.6 Parameters Along Horizontal Well (Dia $=4.28$ in) for Reservoir Permeability

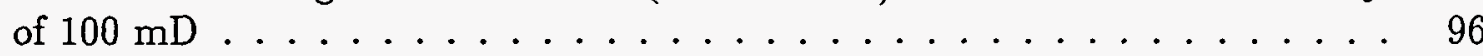


10.7 Production Data of Well (Dia $=6.18 \mathrm{in}$ ) with Reservoir Permeability of $100 \mathrm{mD}$

10.8 Parameters Along Horizontal Well (Dia $=6.18$ in) for Reservoir Permeability

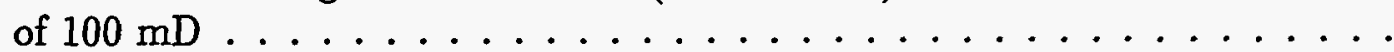

10.9 Reservoir Characteristics and Non-Uniform Gridding in the y-z Plane for the Non-Homogeneous Case . . . . . . . . . . . . . . 100

10.10Production Data of Well (Dia=4.28in) with Reservoir Permeability of 100-500 $\mathrm{mD} \ldots \ldots \ldots \ldots \ldots 1 . \ldots \ldots \ldots$

10.11Parameters Along Horizontal Well (Dia $=4.28$ in) for Reservoir Permeability of $100-500 \mathrm{mD}$. . . . . . . . . . . . . . . . . . . .

10.12Production Data of Well (Dia $=4.28$ in) for Fixed BHP with Reservoir Permeability of $100 \mathrm{mD} \ldots \ldots \ldots \ldots \ldots \ldots \ldots \ldots$

10.13Parameters Along Horizontal Well ( $\mathrm{Dia}=4.28$ in) for Fixed BHP with Reservoir Permeability of $100 \mathrm{mD} \ldots \ldots \ldots$. . . . . . . . . . . . . . 104

10.14Production Data of Well (Dia $=4.28$ in) for Fixed BHP with Reservoir Permeability of $100-500 \mathrm{mD} \ldots \ldots \ldots \ldots \ldots \ldots \ldots$

11.1 Flow Pattern Map for Oil/Gas System at Flowing Conditions for a Horizontal

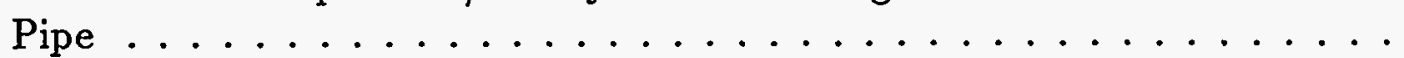

11.2 Pressure Drop due to Friction for Oil/Gas System at Flowing Conditions for a Horizontal Pipe . . . . . . . . . . . . . . .

11.3 In-Situ Liquid Fraction for Oil/Gas System at Flowing Conditions for a Horizontal Pipe . . . . . . . . . . . . . . .

11.4 Flow Pattern Map for Oil/Gas System at Flowing Conditions for a Pipe at $5^{\circ}$ Downward Inclination . . . . . . . . . . . . . . .

11.5 Flow Pattern Map for Oil/Gas System at Flowing Conditions for a Pipe at $5^{\circ}$ Upward Inclination . . . . . . . . . . . . . . . . .

11.6 Flow Pattern Map for Oil/Gas System at Flowing Conditions for a Pipe at $80^{\circ}$ Upward Inclination . . . . . . . . . . . . . .

12.1 Layout of the Marathon Wellbore Model . . . . . . . . . . . . . 117

12.2 Data Analysis of Single-Phase Water Core Flow Experiments . . . . . . . . 119

12.3 Data Analysis of Single-Phase Oil Core Flow Experiments . . . . . . . . 120

12.4 Data Analysis of Single-Phase Oil Flow Experiments with Oil in the Core and through Perforations . . . . . . . . . . . . . . . . . . . . 121

12.5 Pressure Drop Along the Wellbore for Two-Phase Water/Air Flow . . . . . 122

12.6 Pressure Drop Along the Wellbore for Two-Phase Oil/Nitrogen Flow . . . . 123

12.7 Photograph of Liquid Holdup Meter in Position in the 5-15 ft Section of Acrylic Pipe . . . . . . . . . . . . . . . . . . . 125

12.8 DP Measurements During Slug Flow for Nitrogen Inflow Experiment . . . 126

12.9 Liquid Holdup Measurements During Slug Flow for Nitrogen Infiow Experiment127 


\section{List of Tables}

1.1 Comparison of Expected and Actual Horizontal Well Performance for the Ness

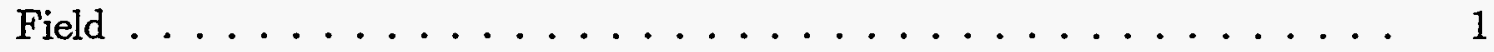

4.1 Eclipse Input Data for Productivity Calculations . . . . . . . . . . . . . 34

6.1 Model Description for Shale Studies . . . . . . . . . . . . 55

7.1 Estimates of Critical Oil Rates for a Single Horizontal Well from Different

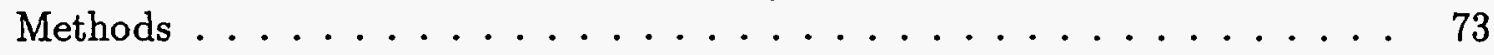

9.1 Estimates of Pressure Drops for the Example Calculation Using Different Cor-

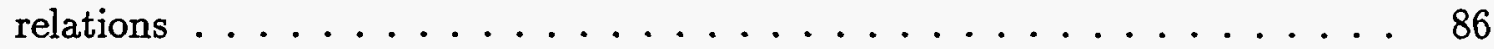

10.1 Summary of the Reservoir Characteristics and Parameters Used in the Cresting Simulation Example . . . . . . . . . . . . . . 90

11.1 Fluid Properties Used in the Mechanistic Model . . . . . . . . . . . . 109 


\section{Introduction}

Horizontal wells (HWs) have provided a major breakthrough in new techniques for developing oil fields; the drilling technology was first pioneered by Elf Aquitaine and the Institut Francais du Petrole (IFP) with application in the offshore Raspo Mare field in the Adriatic (circa 1977). Since then the technology has expanded enormously with one assessment indicating over 800 horizontal wells being drilled in 1991 [1]. Some 4,200 HWs are known to have been drilled to the end of 1993 in the USA. Drilling costs have decreased to be only about 1.3 times those for vertical wells, even with horizontal sections of 2,000 feet or more. Although the drilling techniques in association with Measurement While Drilling have progressed to remarkable accuracy, and in more recent years clearer insights have been established on completion practices, the reliable prediction of the reservoir engineering and performance attributes of horizontal wells remains a severe challenge. There is a tendency for publications to dwell on successful applications; however, the fact that some $50 \%$ of the horizontal wells in the Bakken Field [2] have been economic failures illustrates the fact that performance prediction has significant uncertainties. A revealing article was published on the Ness Field [3] in the North Sea where the findings summarized in Table 1.1 indicate performance badly short of expectation, in spite of careful and detailed reservoir evaluation work. In some situations the performance of HWs has been only moderate, so that the extra drilling and completion costs are only marginally compensated in relation to the more conventional vertical or deviated wells. For example, in the Prudhoe Bay field where reduction of gas coning under an expanding gas cap provides an incentive [4], the use of horizontal wells has not generally been as beneficial as first anticipated. Part of this has been attributed to a rapid build-up in gas/oil ratio after gas breakthrough into the well. In vertical wells, however, techniques for an optimal recompletion of the well to offset gas coning behavior can be used with reasonable confidence, thus the economic benefits for horizontal wells are not always clearly assured. This raises the important question of optimal recompletion strategies for long HWs.

Table 1.1: Comparison of Expected and Actual Horizontal Well Performance for the Ness Horizontal Well Case Study [3]

\begin{tabular}{|lcc||}
\hline & Expected & Actual \\
\cline { 2 - 3 } Sustained Oil Rate BOPD & 10,000 & 2,200 \\
Drawdown, psi & 10 & 200 \\
Time to First Water & 2 Years & $<1$ week \\
\hline
\end{tabular}


A significant factor in assessing the performance of a horizontal well can be associated with pressure drops along the well bore, particularly for the two-phase and three-phase regimes (gas/oil, water/oil, or water/gas/oil). The horizontal disposition plus the transverse energetic entry of fluids from many points in the long section will cause a change in the more conventional flow patterns, as determined from steady state flow far from the entrance section in simple horizontal or vertical pipes. This implies that the standard correlations, like the Beggs and Brill correlation [5] which are usually employed for modeling the wellbore flow performance are likely to be seriously in error for horizontal wells. We will present illustrative calculations for this aspect of horizontal well performance which indicate the possible magnitudes of errors which can arise.

In this report, we review a range of reservoir scenarios in which HWs can be advantageous and discuss some of the modeling problems associated with calculating well connection factors, productivity indices, coning behavior and well two-phase pressure drops. We show illustrative coning calculations and the implications of the well model on distribution of postbreakthrough gas saturations. Such calculations then open up the possibility of determining optimal recompletion strategies and/or additional hydraulic fracturing. 


\section{Advantageous Reservoir Opportunities for Horizontal Wells}

Horizontal wells give the opportunity for substantial increases in productivity and sweep factor per well for any oil displacement process, because the length of their producing contact with the oil zone can be almost as long as we wish. By comparison, for vertical wells, the contact length is restricted by the thickness of permeable reservoir within the oil pay zone. We also have the opportunity to place the horizontal well at a particular level in the reservoir, so that it will be adjacent to the most abundant oil zone; e.g., either at a higher or lower level if there have been gravity segregation effects associated with a previous water or gas drive. However, if a reservoir is highly layered, with essentially no communication between the layers, then vertical wells have an advantage because they will intersect and contact all the layers. Most reservoirs exhibit some general layering properties, but often with some communication. Therefore, heterogeneities make an important impact on the relative advantages of horizontal and vertical wells, and the details of the heterogeneity distributions will also dictate the optimum economic length for a horizontal well. If the number of non-communicating layers is not too large, then a horizontal well may be stepped through these layers as shown in Fig. 2.1. Part of the reservoir engineering optimization problem is then to arrange the length and location of each horizontal section in the most productive manner.

\subsection{Low Permeability Reservoirs}

The increased length of horizontal wells gives clear productivity advantages for low permeability reservoirs. Vertical wells suffer from low productivity, which often is improved to some degree by hydraulic fracturing. The level of improvement will depend on the fracture characteristics. Fracturing will sometimes penetrate adjacent zones or layers containing mobile gas or water, which then prejudices the improvement in oil productivity. Often a tight reservoir will be heterogeneous with areal distributions of very low permeability. Thus, a vertical well may often strike the very tight region; whereas, the horizontal well can intersect both very tight and more productive sands, as shown in Fig. 2.2. The advantage is realized provided the horizontal well is drilled with an orientation orthogonal to the paleoslope of the reservoir geological variations.

\subsection{Reservoirs Sensitive to Gas or Water Coning}

New fields will sometimes have relatively thin oil columns, positioned above an aquifer, or with a gas cap overlying the oil, or sometimes both can occur. Even with quite moderate effective vertical permeabilities, these fields will then incur coning of water or gas into vertical production wells. The only remedy is to close off the part of the vertical well adjacent to the cone and accept a consequent reduction in oil production rate. This type of application with both gas and water contacts is being addressed in the Troll field [6] in the Norwegian 


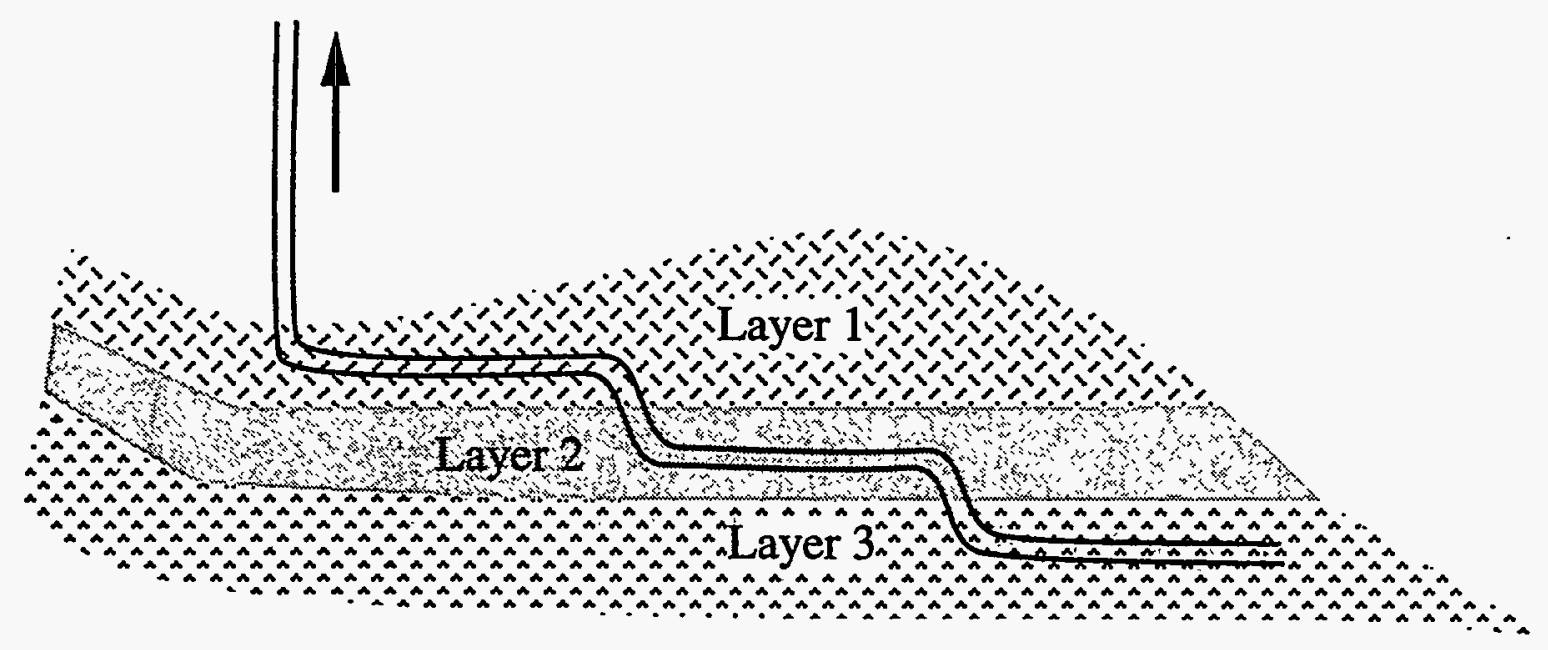

Figure 2.1: Stepped Horizontal Well Used to Produce Three Non-Communicating Reservoir Layers

Sector of the North Sea. Here, there may be advantages in placing the horizontal well just below the water/oil contact to induce inverse coning of oil.

For fields which have been in production for many years, an originally thick oil column have diminished due to the downward movement of the gas/oil contact, to a degree that causes unacceptable GOR's in the production facilities (e.g., this is a central problem in the Prudhoe Bay Field). Horizontal wells placed low in the reservoir at this stage can give reduced coning effects, due to their lower flow rates per unit length, and also give better areal sweep for the remaining oil. However, the cost of further drilling may not merit the delayed benefits of additional oil recovery.

Similar considerations apply to water coning at the later stages of field life. Sometimes there will be the possibility of displacements in both gas/oil and water/oil contacts impacting the decisions on placement of a horizontal well. Depending on the geometrical disposition of the contacts, there may be advantages in using a slant well placed slightly above a curved water/oil contact, as indicated in Fig. 2.3. This alternative has been considered for the lower reservoir in the large Statfjord field in the Norwegian Sector of the North Sea.

\subsection{Heterogeneous Reservoirs}

We have already drawn attention to heterogeneity effects in connection with tight reservoirs in Section 2.1. Similar considerations apply to water flooding or gas flooding reservoirs with particular patterns of heterogeneity, for most depositional environments. The recognition of preferred directions for heterogeneity is now giving planning opportunities in field management, where the flood direction is chosen to maximize the sweep efficiency in a non-uniform, non-isotropic reservoir. Similarly in choosing in-fill drilling patterns in older fields, the details of anisotropic characteristics of the heterogeneity may dictate a preferred placement and sweep orientation for new vertical wells. Horizontal wells give further opportunity to increase the horizontal sweep factor by accessing less permeable regions which would tend to be bypassed in a vertical well configuration. Horizontal wells could also be used as injectors, in addition to their application as producers. The areal sweep potential has to be assessed relative to potential losses or gains associated with vertical sweep efficiency in a layered system. Fig. 2.4 illustrates a scenario for placement of horizontal wells for sweeping 


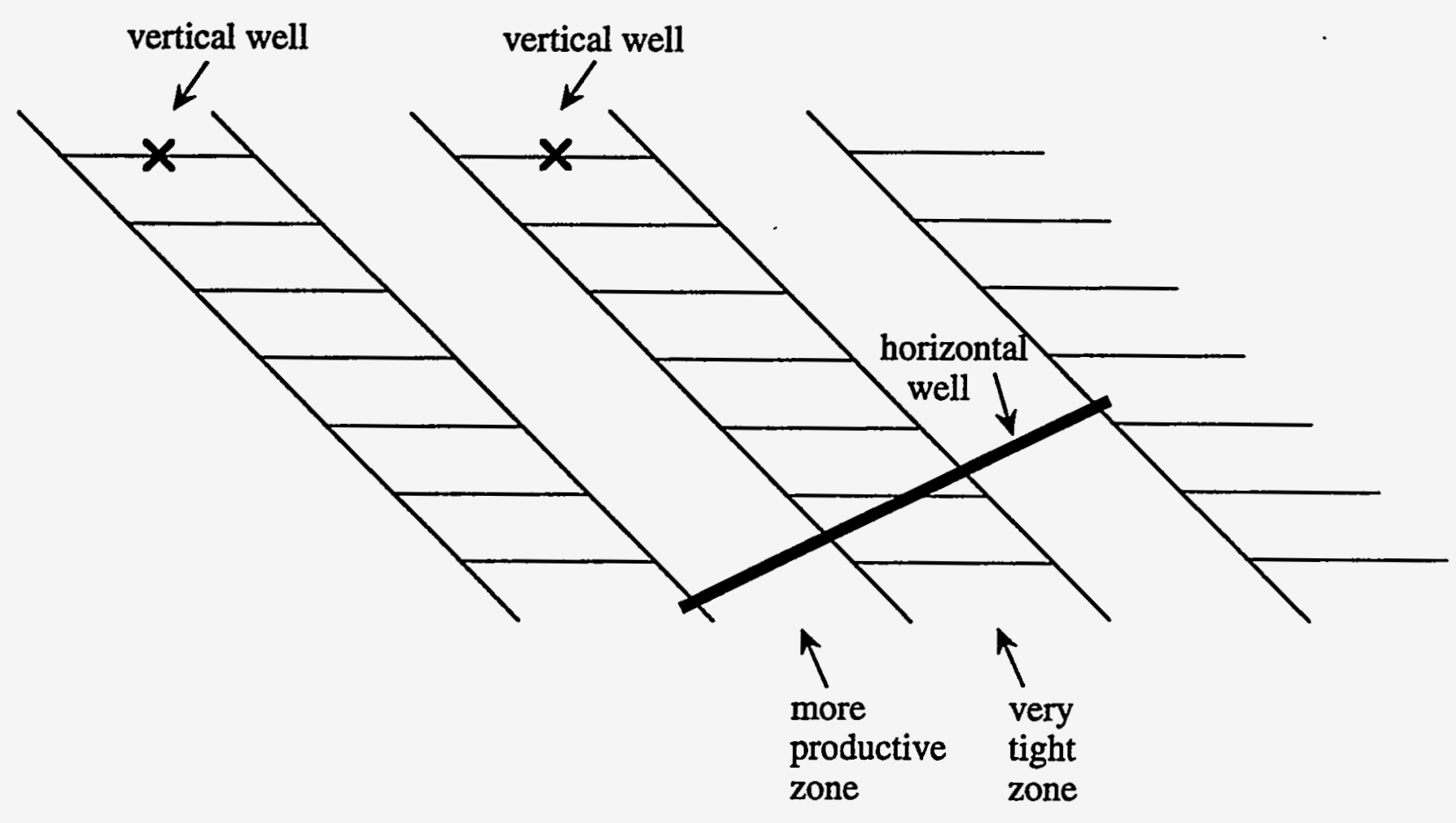

Figure 2.2: Advantageous Orientation of Horizontal Well to Ensure Contact with Productive Zones in Tight Formations

a conceptual braided stream environment in a submarine fan, relative to a corresponding line drive with vertical wells. The use of dual horizontal producers and injectors has been examined for the New Hope reservoir in East Texas [7].

\subsection{Faulted and Fractured Reservoirs}

Faulted and fractured reservoirs are reservoir types with extreme forms of heterogeneity. In Fig. 2.5a we illustrate a faulted formation in which the horizontal well has been positioned to intersect a sequence of fault blocks, thereby ensuring a distribution of drainage from all these blocks. (Similarly at a smaller scale we can drain simultaneously from matrix blocks in a fractured reservoir.) The corresponding sweep capability of a vertical well is limited to a single fault block, although this depends on the degree to which the faults can be conducting. However, if there is also a nearby water/oil or gas/oil contact (Fig. 2.5b), conducting faults can magnify the influence of cusping drawdown of the contact into the horizontal well. Horizontal wells are being successfully applied to the production of the fractured Austin Chalk [8] in Texas. The relative productivities of the fault or fracture blocks will depend on their average permeability characteristics, and also on the pressure drop behavior along the bore of the horizontal well. A preferred sequence would be to orientate the well so its entrance end is near a less permeable block, and its closed end adjacent to a more permeable block. There will be strong interactions between the reservoir permeability distribution, reservoir to well flows in terms of drawdown and local fluid saturations, and 


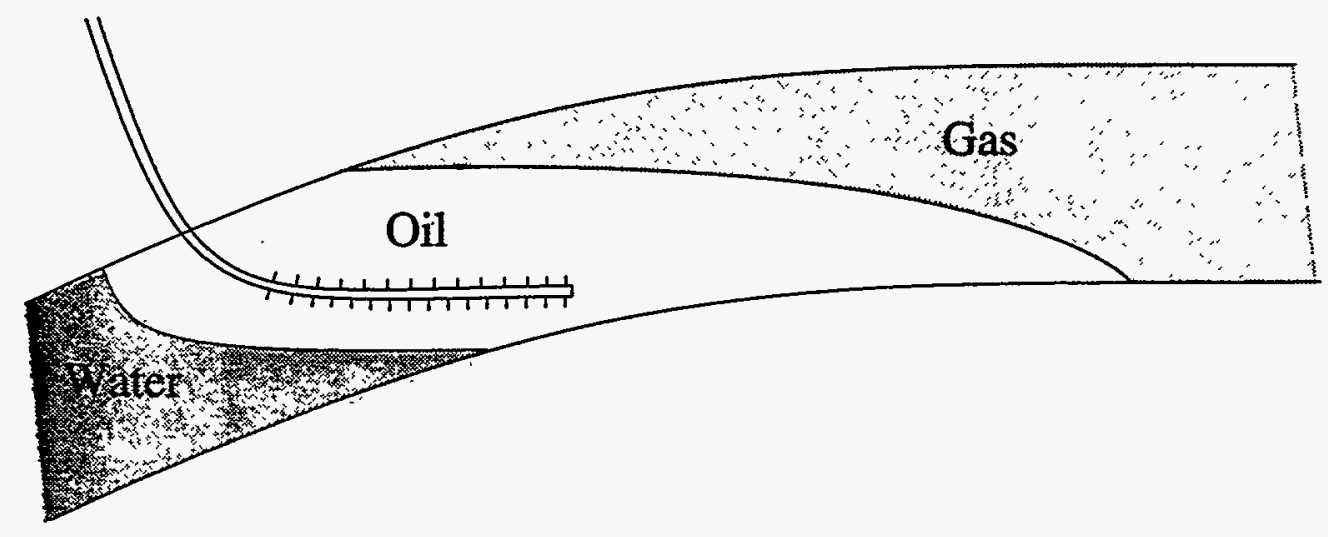

Figure 2.3: Optimum Placement of Horizontal/Slant Well to Avoid Breakthrough from Gas/Oil and Water/Oil Contacts

two-phase pressure drops along the well. We will illustrate this behavior in Section 6 .

Fractures can be introduced artificially into horizontal wells by hydraulic stimulation, as with vertical wells. If the well is drilled perpendicular to the principal stress direction, these fractures can be propagated orthogonal to the well at selected intervals. The geometry is then similar to the faulted problem described above, except the induced fractures will have a distribution of finite lengths (Fig. 2.6a). Their geometry will approximate long thin ellipsoids. Alternatively, if the well is drilled parallel to the principal stress direction, the induced fractures can be propagated in a vertical plane along the well (Fig. 2.6b). Instead of drilling the stepped well shown in Fig. 2.1, a long well drilled in the layer 2 could be fractured into layers 1 and 3. The technology for selectively packing off intervals and performing hydraulic fracture stimulations in horizontal wells has been developed by Maersk Oil and Gas in connection with the Dan Field [9], which is a chalk field in the Danish sector of the North Sea. The completion intervals can also be controlled during production using a sliding side-door technique to isolate sections of the well which are producing too much gas or water. Performance prediction for such systems is extremely complicated.

\subsection{Potential Miscible Gas Applications}

Miscible gas displacement using either $\mathrm{CO}_{2}$ or enriched hydrocarbon gas provides the only economically and technically demonstrated process at this time for enhanced oil recovery for a number of large nearly depleted fields in the USA (e.g., the Wasson and Slaughter fields for $\mathrm{CO}_{2}$ in West Texas, and the Prudhoe Bay field for enriched natural gas). Miscible gas provides many other opportunities, for the present and the future (the OGJ EOR review [10] of April 1992 lists over 50 field projects in the USA). Because of density differences relative to oil and water, miscible gas displacement can be subject to severe gravity override, giving a gas-sweep pattern resembling an inverted cone near a vertical injection well (see Fig. 2.7a). This behavior depends on the effective vertical permeability, and the possible predominance of layering effects in the reservoir. Thus, a thin layer with large permeability may act as a thief zone to miscible gas and again limit the achievable vertical sweep. Horizontal wells give the opportunity to position both injection and production wells at an advantageous level in the reservoir, so as to maximize the overall vertical sweep (as shown in Fig. 2.7b for 
(a) VERTICAL WELLS

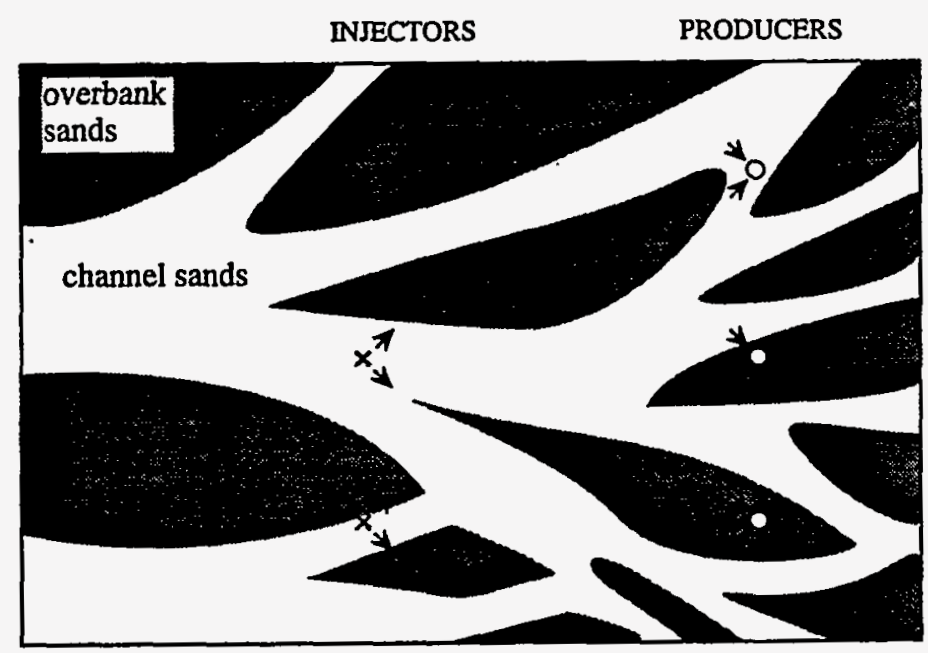

(b) HORIZONTAL WELLS

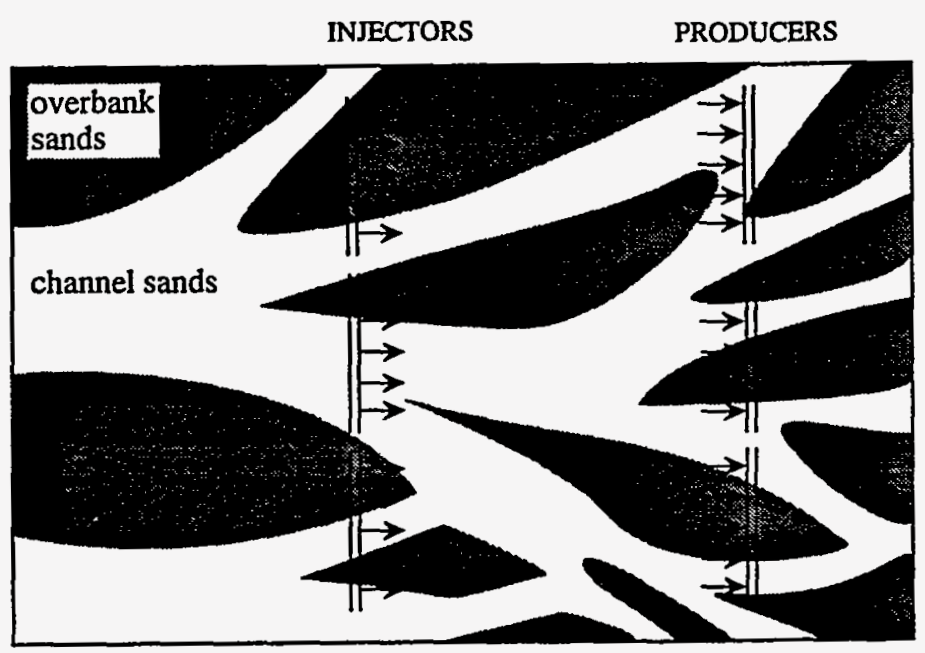

Figure 2.4: Improved Areal Sweep for Braided Stream Environment with Horizontal Wells

the gravity segregation case). The optimum placement will depend on whether the miscible gas is to be injected as a single large slug or in a water-alternating gas (WAG) application. Associated benefits for miscible EOR are also derived from horizontal injectors in terms of areal sweep, as shown in Fig. 2.7d, where the sweep factor is better than that obtained from a corresponding arrangement of vertical wells, illustrated in Fig. 2.7c. Reservoir heterogeneities will again strongly influence the relative merits of vertical and horizontal wells, and how the horizontal wells should be positioned for maximum advantage. The modeling problems are even more difficult for this application, because compositional effects and phase changes must be considered in the coupling behavior with the well. 
Drains multiple fault blocks

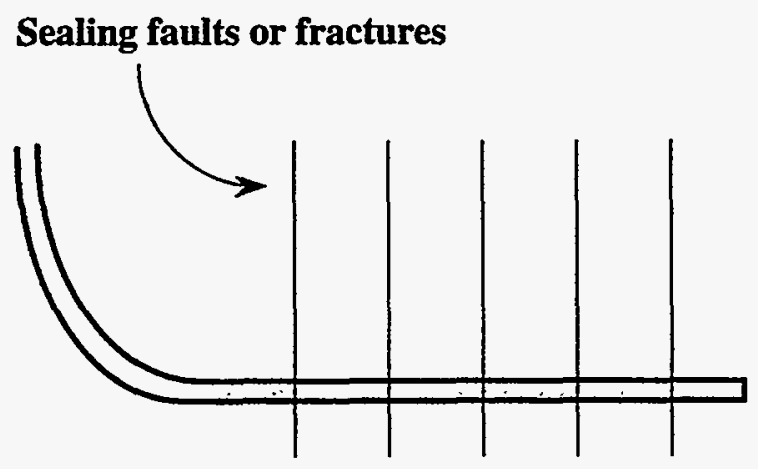

Accelerated gas breakthrough

\section{Conducting faults or fractures}

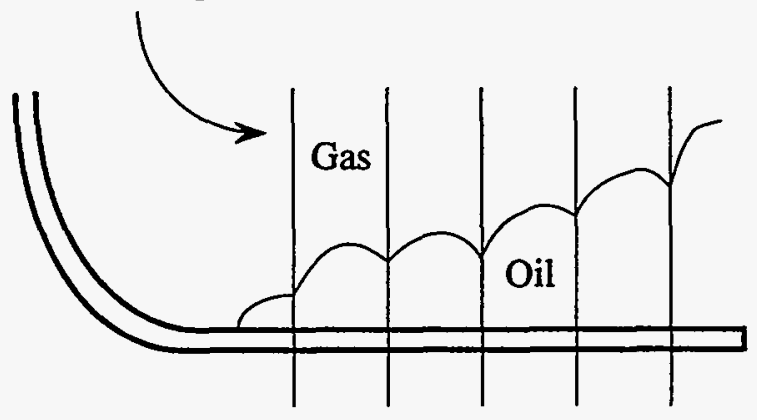

Figure 2.5: Considerations of Effects of Faults or Fractures on Performance of Horizontal Wells

\subsection{Thermal Recovery of Heavy Oil}

All the previous discussion concerning reservoir geological characteristics, and factors relating the relative desirability of vertical and horizontal wells, come into play in thermal applications for heavy oil production. The increased injectivity and productivity characteristics should give an added incentive for horizontal wells in heavy oil fields. There are a number of possible arrangements to be considered in both steam injection or in-situ combustion applications. One of the significant concerns with both of these thermal processes has been with questions of the effective vertical and horizontal sweep factors that will be achieved in a system which has a severely unfavorable mobility ratio. Horizontal wells should offer significant improvements.

An interesting alternative on positioning dual horizontal injection and production 
A)

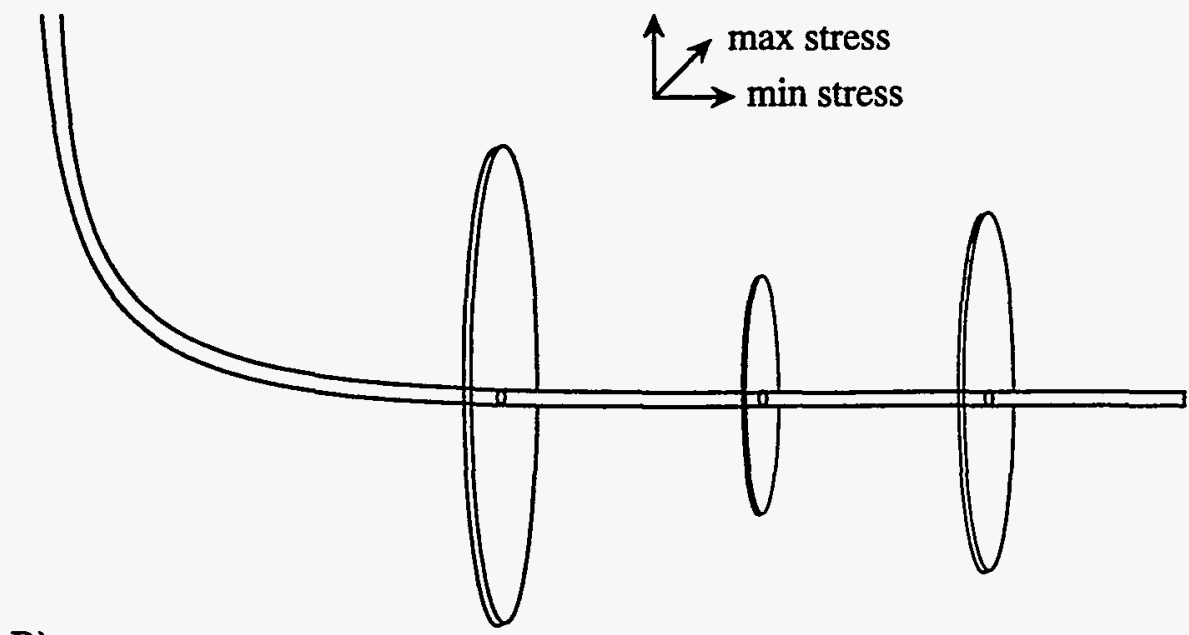

B)

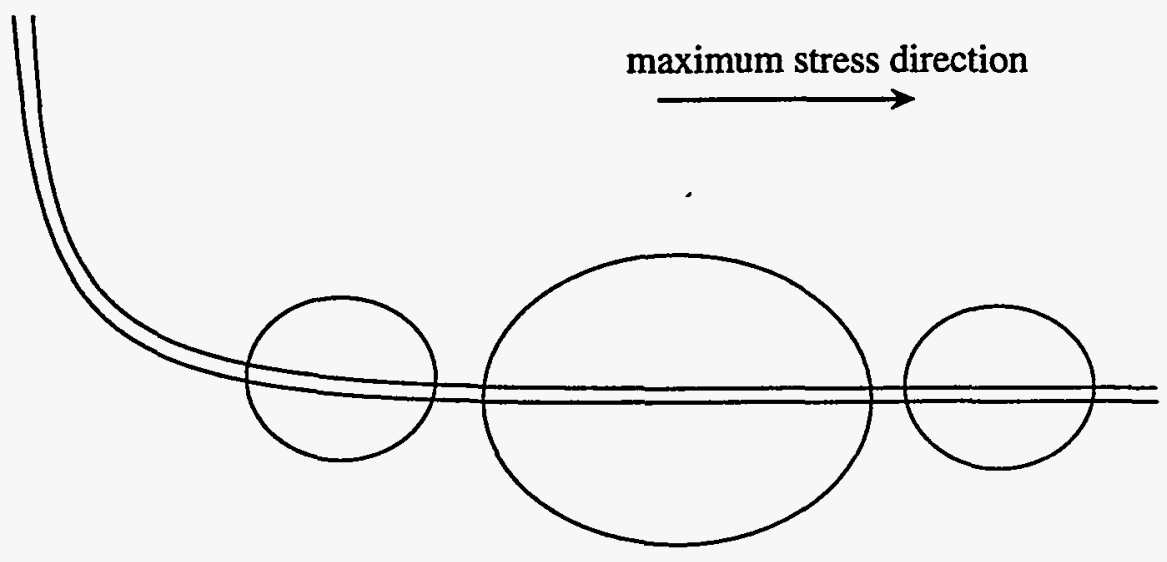

Figure 2.6: Horizontal Well with (A) Transverse and (B) Parallel Multiple Hydraulic Fractures

wells for thermal recovery has been to place the injector at a high level in the reservoir to create a steam attic, which then allows the hot oil to drain downwards towards the horizontal producer at a low level [11]. The possibility of a heat-up and pressure cycle followed by a production cycle may be considered with this concept. Horizontal wells may also have application in steam injection into fractured carbonate reservoirs containing heavy oil (e.g., the Carbonate Triangle in Canada). Such an application has been reported for the Lacq Superieur Field [12] in France. 


\section{VERTICAL SWEEP}

(a) Cone about Vertical Well

(b) Crest along Horịzontal Well
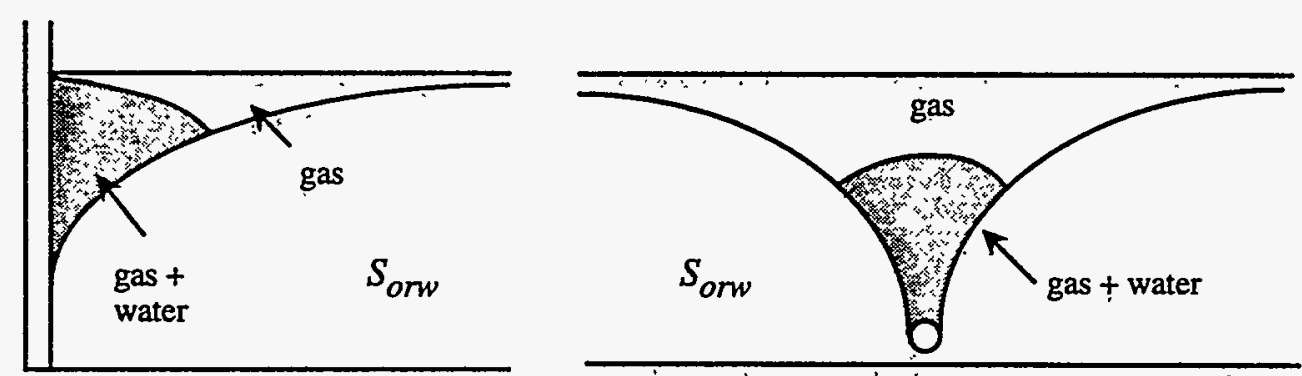

\section{AREAL SWEEP}

(c) Verticạl Wells - strong cusping

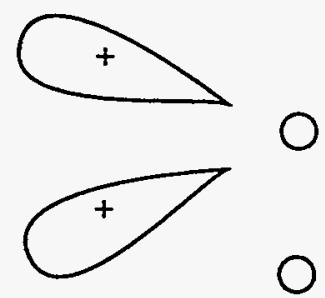

producer

$+$

injector (d) Horizontal Well - reduced cusping

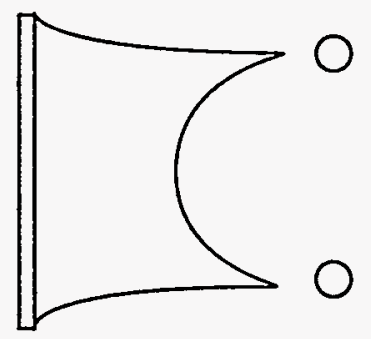

Figure 2.7: Advantages of Horizontal Wells as Injectors in Miscible Gas Applications 


\section{Factors Influencing the Computation of Well Indices (Review: Tasks 1, 2 and 3)}

We will first review some of the modeling issues associated with predicting the performance of horizontal wells under single-phase and two-phase flow conditions, recognizing that some of the applications referred to above will present even more difficult three-phase flow issues. Most black oil simulators used today can represent horizontal wells in three dimensions within the geometrical limitations imposed by a fairly coarse Cartesian mesh. The need for acceptance of a coarse mesh becomes more severe when the simulation model is set up to represent either the whole field, or a large section of that field.

\subsection{Regular Cartesian Grids}

In order to join the well to the Cartesian grid cells within the grid blocks in which the well is located (well blocks), it is usual to use a single-phase productivity index based on the concept of an effective radius $r_{o}$ at which the cell pressure $P_{o}$ applies. Most of the equations given in this section apply to a two-dimensional grid orthogonal to an infinite well in the $y$-direction. Under single phase flow, the flow rate into the well $q$ for this block is given by

$$
P_{o}-P_{w}=\frac{q \mu}{2 \pi\left(k_{z} k_{x}\right)^{\frac{1}{2}} \Delta y}\left(\ln \frac{r_{0}}{r_{w}}+S\right)
$$

where $r_{w}$ and $P_{w}$ are the well radius and pressure, $k_{x}$ and $k_{z}$ are horizontal and vertical permeabilities, and $\Delta y$ the block interval size along the well. $\mu$ is the fluid viscosity and $S$ the skin factor. An important contribution by Peaceman [13] was to derive the following expression for $r_{o}$

$$
r_{\circ}=\frac{0.28\left[\left(k_{z} / k_{x}\right)^{\frac{1}{2}} \Delta x^{2}+\left(k_{x} / k_{z}\right)^{\frac{1}{2}} \Delta z^{2}\right]^{\frac{1}{2}}}{\left(k_{z} / k_{x}\right)^{\frac{1}{4}}+\left(k_{x} / k_{z}\right)^{\frac{1}{4}}}
$$

This equation is normally used in most black oil simulators. Its validity depends on the assumptions of single-phase flow, infinite well length with $k_{x}=k_{y}$, uniform permeability, uniform grid, and this isolated well must not be too close to any boundaries or other blocks

that contain wells. Peaceman suggests that the top and bottom boundaries will not be limiting provided

$$
\frac{\Delta x}{\Delta z}\left[\frac{k_{z}}{k_{x}}\right]^{\frac{1}{2}} \leq 0.9 \frac{\min \left(z_{w}, h-z_{w}\right)}{\Delta z}
$$

where $z_{w}$ is the height of well above the bottom of the pay zone. Eq. (3.2) can be generalized to a number of wells within a rectangular reservoir region if the pressures on the boundary are assumed to conform to a summation of $l n$-functions.

Further analysis of the problem of an accurate choice for $r_{o}$ has been undertaken by Babu et al. [14]. Their approach was to derive an analytical solution for steady-state 
pressure behavior as a function of flow rate, and then to compare this with an exact solution of the corresponding finite difference problem. Their solution for $r_{0}^{*}$ can be presented in the form

$$
\ln \frac{r_{o}^{*}}{h}=\frac{\pi w}{6 n_{x}^{2} h}\left[\frac{k_{z}}{k_{x}}\right]^{\frac{1}{2}}+\frac{1}{4} \ln \frac{k_{x}}{k_{z}}-\ln \left[2 \pi \sin \frac{\pi z_{w}}{h}\right]-S_{x z}-B_{E}
$$

where $h, w$ are the height and width of the reservoir and $n_{x}$ the number of $x$-grid blocks. $S_{x z}$ and $B_{E}$ are functions which depend on the geometry and the number of grid blocks in the $x$ and $z$-directions as defined below.

$$
\begin{gathered}
S_{x z}=\left(\frac{\pi}{n_{z}}\right) \sum_{n=1}^{n_{z}-1} \cos ^{2}\left(\frac{\pi n \lambda}{2 n_{z}}\right)\left(1+x_{n}^{-\nu}\right)\left(1+x_{n}^{\nu-2 n_{x}}\right) / \\
{\left[\sin \left(\frac{\pi n}{2 n_{z}}\right)\left(1+\alpha_{n}^{2}\right)^{\frac{1}{2}}\left(1-x_{n}^{-2 n_{x}}\right)\right]} \\
\alpha_{n}=\alpha \sin \frac{\pi n}{2 n_{z}} \\
\alpha=\frac{w}{h}\left(\frac{n_{z}}{n_{x}}\right)\left(\frac{k_{z}}{k_{x}}\right)^{\frac{1}{2}} \\
x_{n}=\left[\alpha_{n}+\left(1+\alpha_{n}^{2}\right)^{\frac{1}{2}}\right]^{2} \\
\nu=2 i_{w}+1 \\
\lambda=2 j_{w}+1
\end{gathered}
$$

where $i_{w}, j_{w}$ are the integer coordinates of the well location.

$$
B_{E}=\ln \left(1-E_{1}\right)+\frac{1}{2} \ln \left[1-2 \cos \left(\frac{2 \pi z_{w}}{h}\right) E_{1}+E_{1}^{2}\right]
$$

where

$$
E_{1}=\exp \left[-\frac{2 \pi \min \left(x_{w}, w-x_{w}\right)}{h}\left(\frac{k_{z}}{k_{x}}\right)^{\frac{1}{2}}\right]
$$

$B_{E}$ is usually small, unless the well location $x_{w}$ is placed close to the $x=0$ or $x=w$ boundaries. Equation (3.1) is valid when $w / \sqrt{k_{x}} \geq 0.75 h / \sqrt{k_{z}}$

In a further modification, Brigham [15] points out that Eq. (3.4) neglects a necessary transformation on the wellbore radius for the effects of anisotropy. This implies a correction to $r_{o}$ which takes the form

$$
r_{o}=\frac{r_{0} *}{\frac{1}{2}\left[\left(k_{z} / k_{x}\right)^{\frac{1}{4}}+\left(k_{x} / k_{z}\right)^{\frac{1}{4}}\right]}
$$

These equations could be programmed into black-oil simulators. Although less dependent on the position chosen for the boundaries of the reservoir, the results are still subject to all the other limitations expressed for the Peaceman equations. In particular these relations do not tell us how to deal with wells of finite length in heterogeneous reservoirs, with general anisotropy $\left(k_{x} \neq k_{y}, k_{x y} \neq 0\right.$, etc.), variable grid spacing, multiple interacting wells, and two-phase flow. 


\subsection{More Advanced Gridding Concepts}

The more accurate approach is to study the well performance using variably spaced grids [16], specially chosen to give closer spacings near the well, so that the formula for $r_{0}$ becomes less critical. One scheme is the hybrid method [17], which uses a cylindrical mesh near the well combined with the Cartesian grid at a suitable distance. This leads to non-orthogonality of the gridding scheme near the joining region, with uncertain errors in the finite difference formulation. Another alternative is for the mesh lines of a local fine grid to be terminated abruptly at positions some distance away from the well. These two schemes are illustrated in Fig. 3.1. Mesh line deletion techniques have not always justified their apparent attractions in terms of accuracy versus speed. A third alternative which has been studied by Palagi [18] at Stanford University rests on the use of Voronoi meshes. An example of this type of gridding is shown in Fig. 3.2. It is sometimes referred to as the PEBI method [19] (perpendicular bisections).

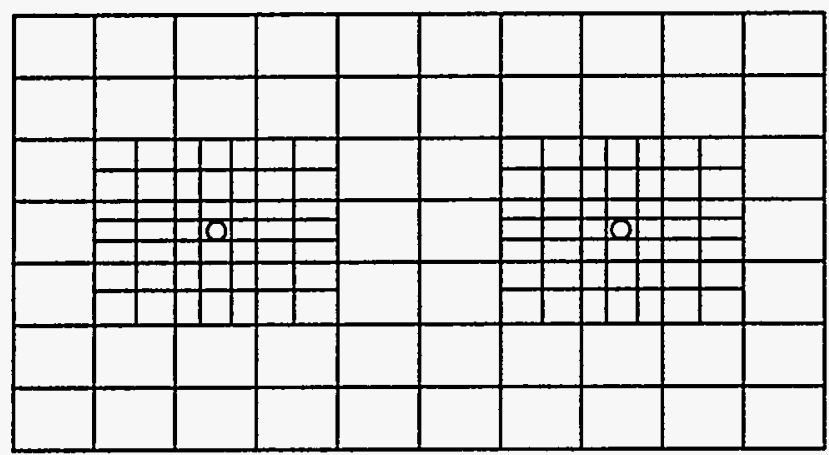

a) Local Grid Refinement
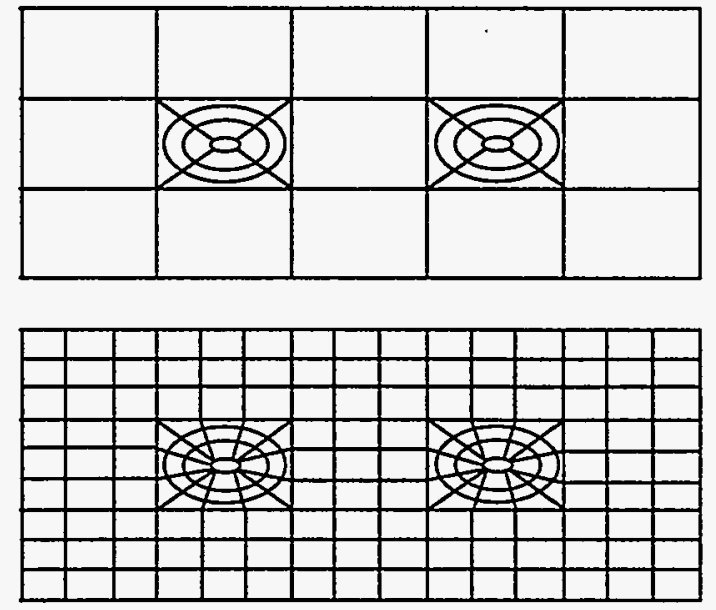

b) Hybrid Grids

Figure 3.1: Special Gridding Schemes

Palagi and Aziz [20] have developed an extension of the Peaceman method for evaluating $r_{o}$ in nonuniform gridded problems. Their approach is an exact analytic solution of the transient equation for the homogeneous problem, using a superposition algorithm with an arbitrary number of wells with representative fixed flow rates, and a numerical solution of the diffusion equation based on the actual grid to be used in simulation. The solution for vertical wells is achieved by the following steps evaluated at pseudo steady-state conditions. For any well $m, r_{o m}$ is obtained from

$$
\ln \left[\frac{r_{o m}}{r_{w m}}\right]=\frac{2 \pi k h}{q_{m} B_{o} \mu_{o}}\left[p_{o m}-p_{w m}\right]
$$




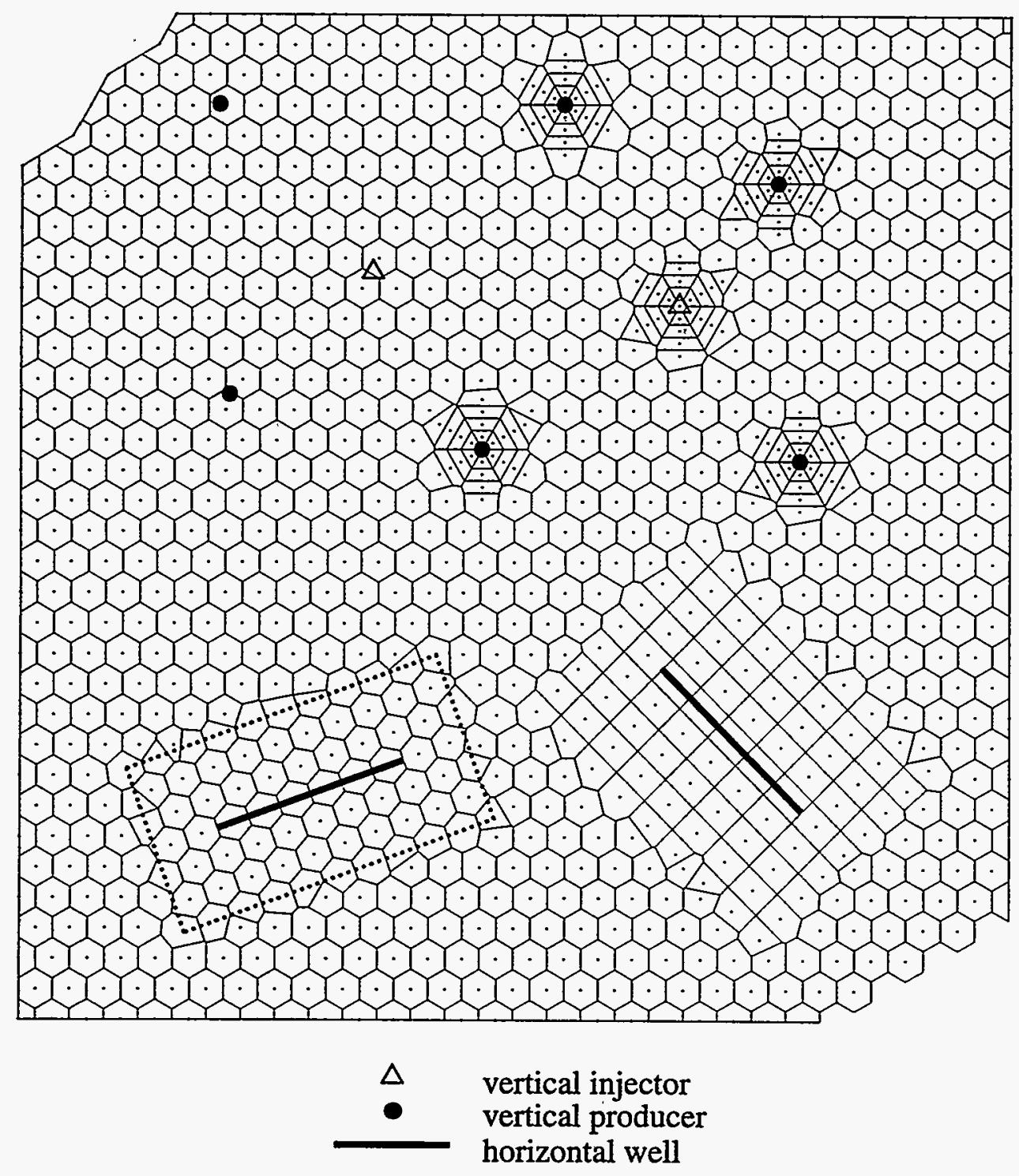

Figure 3.2: Generalized Voronoi Grid for Representation of Horizontal and Vertical Wells [18] 


$$
=\frac{2 \pi k h}{q_{m} B_{o} \mu_{o}}\left\{\left[\bar{p}-p_{w m}\right]-\left[\bar{p}-p_{o m}\right]\right\}
$$

where $\bar{p}(t)$ is the average reservoir pressure. The first term is given by the sum of two analytical results:

$$
p_{\text {in }}-\bar{p}=\frac{B_{o} \mu_{o} q_{t o t} t_{D}}{k h}
$$

where $p_{i n}$ is the initial pressure, $q_{t o t}=\sum q_{j}$ and $t_{D}=k t / \phi \mu c_{t} x_{e} y_{e}$, and the second result by

$$
p_{i n}-p_{w m}=\frac{B_{o} \mu_{o}}{2 \pi k h} \sum_{j} q_{j} p_{D j m}^{(t)}
$$

$p_{D j m}$ is the dimensionless pressure at well $m$ due to the production rate at well $j$ (including their images). The dimensionless pressure functions have the form

$$
p_{D j m}\left(r_{D j m}, t_{D}\right)=-\frac{1}{2} E_{i}\left(-\frac{r_{D j m}^{2}}{4 t_{D}}\right)
$$

where $r_{D j m}=r_{j m} / r_{w j}$ is the dimensionless distance between wells $j$ and $m$. The summation over $j$ includes as many images as needed for reasonable convergence. $\vec{p}(t)-p_{o m}(t)$ required as the second term in Eq. (3.10) is evaluated numerically from a solution of the transient pressure equation using the consistent grid to be deployed in the reservoir simulation. This procedure can also include Voronoi grids, or any irregular grid pattern we may choose, and could be programmed as a pre-processor in reservoir simulators.

Palagi and Aziz investigated simple limiting forms for $r_{o}$ which can be used when the analytical solution is close to the form $\ln \left(r / r_{w}\right)$, i.e. non-interacting wells. The definition of transmissibility through a cell face connecting Voronoi points, $i, j$ is

$$
T_{i j}=\left(\frac{k b f_{c}}{d}\right)_{i j}
$$

where $b_{i j}$ is the length of the cell face, $f_{c i j}$ is an optional transmissivity correction factor for radial flow, and $d_{i j}$ the distance between $i$ and $j$. Thus for the finite difference solution with well production rate $q_{i}$ at position $i$

$$
q_{i}=\frac{1}{\mu} \sum_{j} T_{i j}\left(p_{j}-p_{o}\right)
$$

Equating the analytical and numerical steady-state solutions yields

$$
\ln r_{o}=\left[\sum_{j}\left(f \frac{b}{d}\right)_{i j} \ln d_{i j}-2 \pi\right] / \sum_{j}\left(f_{i} \frac{b}{d}\right)_{i j}
$$

When the Voronoi grid is a regular polygon, this becomes 


$$
r_{0}=d_{i j} \exp \left(\frac{-2 \pi}{N f_{c i j} \tan \left(\frac{\pi}{N}\right)}\right)
$$

where $N$ is the number of cell faces. This gives $r_{o}=0.208 \Delta x$ for a square grid with $f_{c}=1.0$, which is the simplest form of Peaceman model.

The derivation of Eq. (3.16) depends on the assumption that the finite difference values for the points $p_{j}$ will be equal to the values given by the analytic solution. This breaks down for highly asymmetric grids, where in the $x$-direction for $\Delta x \ll \Delta y$, the numerical solution in this direction can have significant error. For general asymmetric Voronoi cases, better results are obtained by basing the approximation for $r_{0}$ on the cell face $j *$ furthest from point $i$. The recommended equation is

$$
r_{0}=d_{i j *} \exp \left(\frac{-\theta_{i j *} d_{i j *}}{f_{c i j *} b_{i j *}}\right)
$$

where $\theta_{i j *}$ is the angle subtended by the face $i j *$ at point $i$. The optional transmissivity correction factor $f_{c}$ can be used to modify transmissivities of grid blocks in finite difference models which are experiencing essentially radial flow, such as a well block. The effective radius of a well block is given by $r_{e}=\sqrt{\frac{A}{\pi}}$, where $A$ is the block area, and the equivalent radius $r_{c}$ of a radial block at which the pressure $P_{i}$ acts is given by

$$
r_{c}=r_{w} \exp \left[\frac{r_{e}^{2}}{r_{e}^{2}-r_{w}^{2}} \ln \frac{r_{e}}{r_{w}}-\frac{1}{2}\right]
$$

To obtain the correct interblock radial flow, the transmissivity factor should be

$$
T_{i j}=\frac{\theta_{i j} k}{\ln \left(d_{i j} / r_{c i}\right)}
$$

which comparing with Eq. (3.14) gives

$$
f_{c i j}=\frac{\theta_{i j}(d / b)_{i j}}{\ln \left(d_{i j} / r_{c i}\right)}
$$

If the radial correction is applied, Eq. (3.18) becomes $r_{0}=r_{c i}$, which is appropriate for radial flow. However, in most finite difference codes the correction $f_{c}$ is not applied (i.e., $f_{c}=1.0$ ), and then Eq. (3.18) should be used omitting $f_{c}$. Fig. 3.3 shows computed correction factors for radial flow, which indicate weak dependence on the ratio of radii, but strong dependence on $\theta$. These correction factors can also be used for grid cells experiencing radial flow adjacent to well blocks. Fig. 3.4 indicates coarse and fine Cartesian grids combined with Voronoi grids adjacent to an injection well and a production well, with equal rates in a confined reservoir. The use of the $f_{c}$-corrections on the Voronoi grids dramatically improves the coarse grid result, as shown in Fig. 3.5, and also has a useful influence on the fine grid result.

The most accurate results with Voronoi grids for vertical wells are obtained when Eq. (3.10) is used (i.e. interacting wells taken into account). The approximations from Eqs. (3.16) and (3.18) give the same results for isolated wells with regular polyhedra, but as the grid aspect ratio becomes more asymmetric, Eq. (3.18) gives better results than Eq. (3.16). The computation of well indices discussed above all relate to wells perpendicular to 


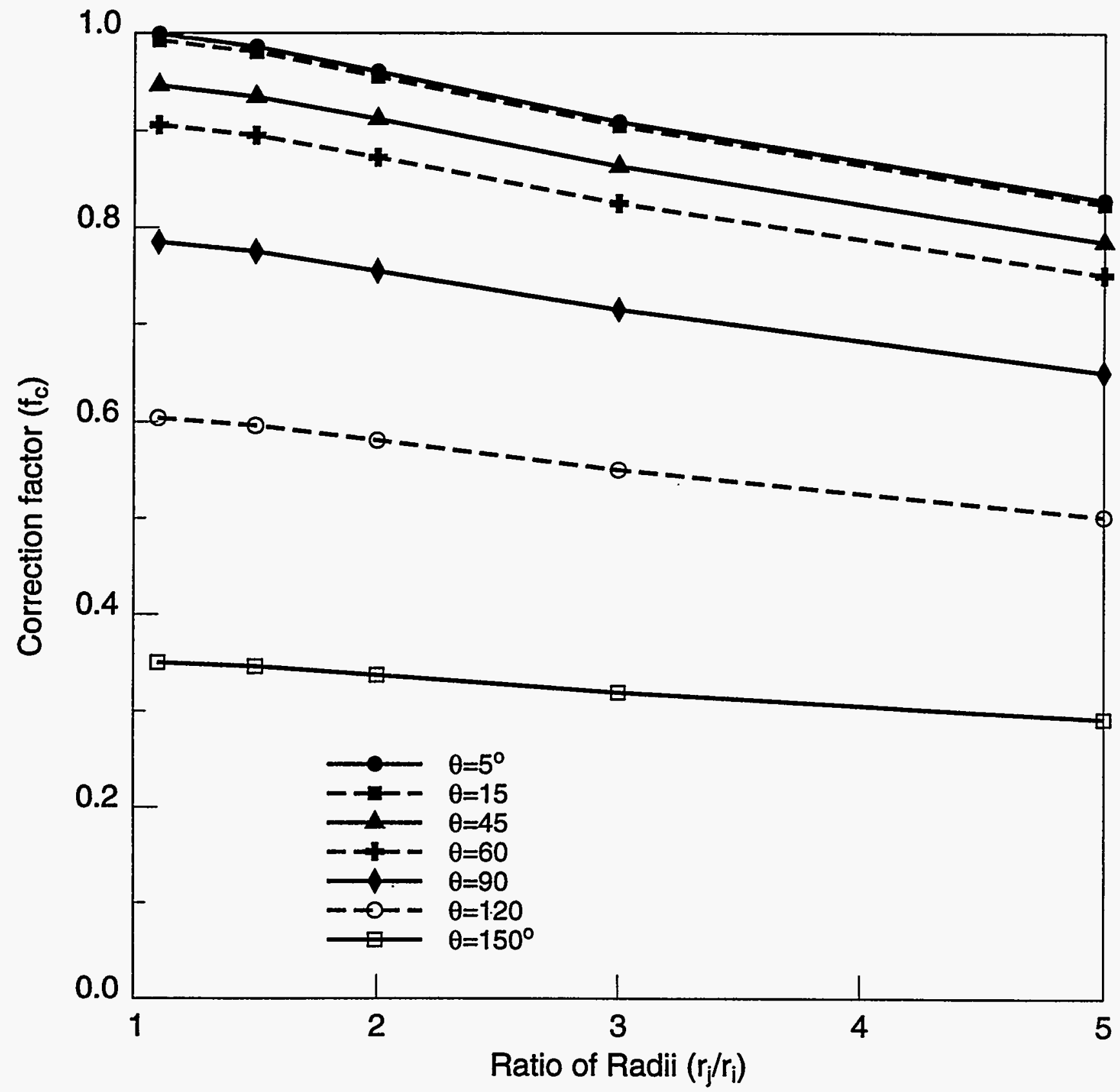

Figure 3.3: Correction Factor $\left(f_{c}\right)$ for "Cylindrical" Modules [18] 


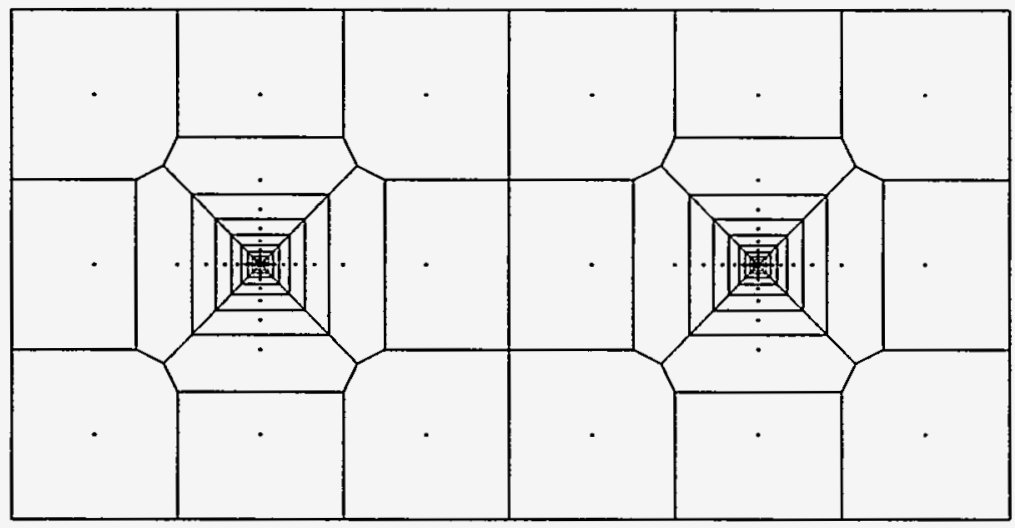

(a) Coarse Cartesian grid (6x3) plus 2 Voronoi (8x4).

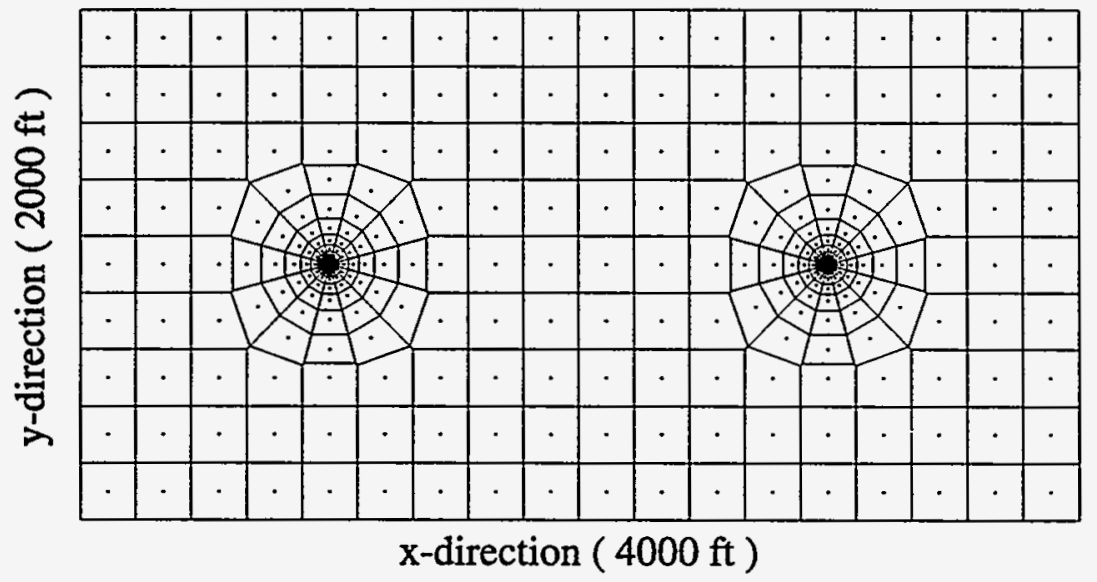

(b) Fine Cartesian grid (18x9) plus 2 Voronoi $(8 \times 12)$.

Figure 3.4: Hybrid-Cartesian Voronoi Grids for Example Problem [18] 


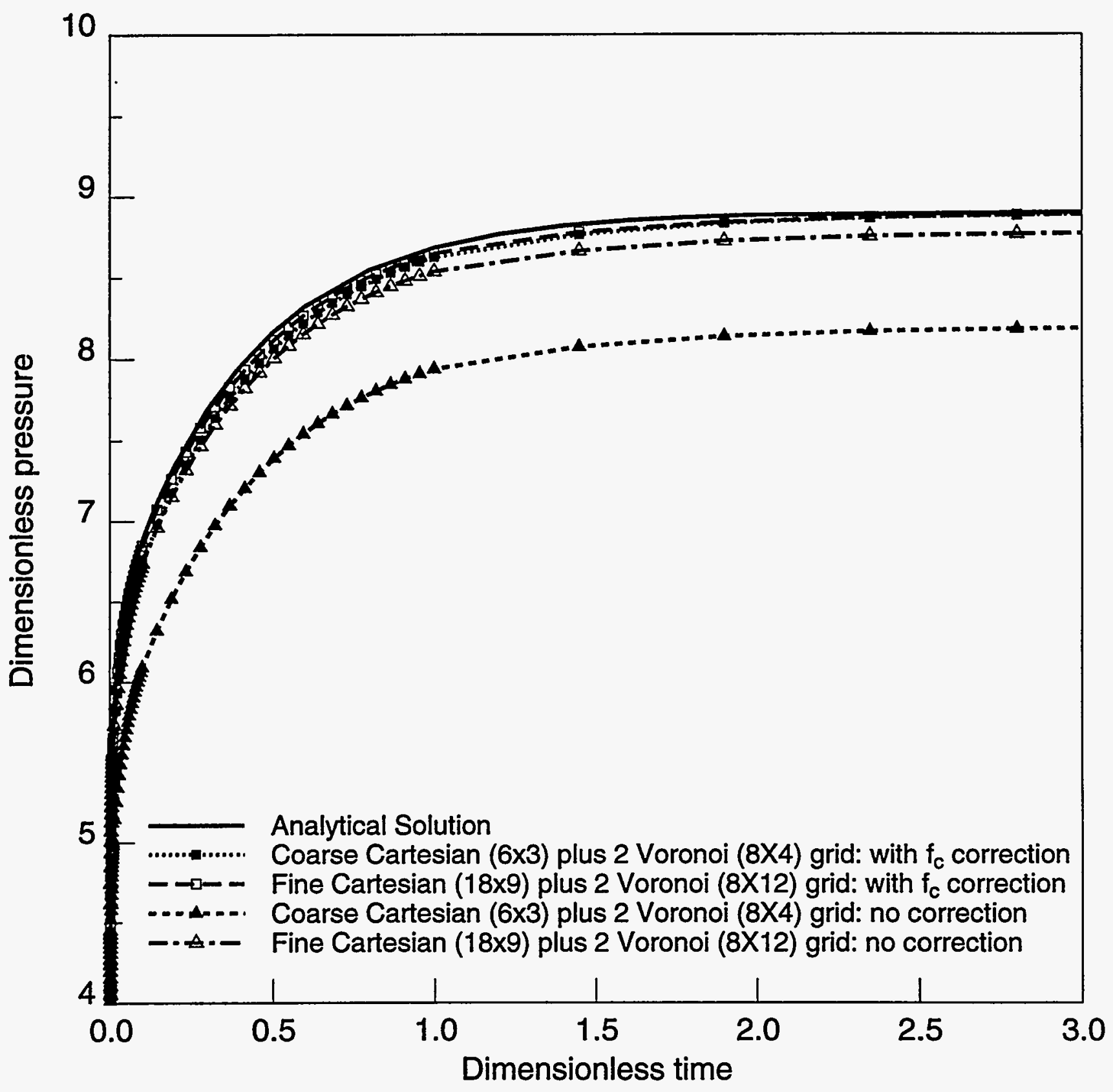

Figure 3.5: Numerical and Analytical Results for Example Problem [18] 
the plane of the 2D-gridding arrangement. For horizontal wells in 3D, the conventional use of a Voronoi grid with constant planar intervals in $\Delta z$ (see the hexagonal example shown in Fig. 3.6) implies the need for special formulae for $r_{o}$ which take account of $k_{z}<k_{x} \neq k_{y}$. A basis for developing an approximate result using the Peaceman equation (3.2) is indicated in Fig. 3.6. This type of application does not appear to have been studied, and its extension to heterogeneous two-phase flow problems with general 3D-Voronoi grids raises research issues for the future.

\subsection{Factors Influencing Well Productivity}

The previous discussion of computation of well indices has been restricted to cases where the well length is infinite in the y-direction. There exits a number of analytic expressions for calculating the steady-state productivity index of a horizontal well with finite length of which one of the earlier results is that due to Giger [21]. These solutions require a constant pressure condition on the outer surfaces of a drainage volume encompassing the well. Since a horizontal well, because of its usually long length, occupies a significant portion of the drainage volume, the constant pressure boundary condition is rarely satisfied. Therefore, the steady-state productivity should only be looked upon as a rough estimate of the actual productivity for a horizontal well. For an anisotropic reservoir, Joshi $[22,23]$ gives an expression for the well productivity index by modifying his isotropic expression by the scaling factor $\beta=\sqrt{k_{h} / k_{v}}$. In another more recent analysis, Economides et al. [19] give a somewhat different analytic solution for the productivity of a horizontal well centrally located in a finite anisotropic 3D-reservoir, with no-flow at top and bottom boundaries. They show for 3D that constant in-flux along the well is a better approximation than constant pressure at the well surface.

Usually the more appropriate boundary condition on all the outer surfaces of the drainage volume containing a horizontal well will be the no-flow condition. The pseudosteady state solutions for the productivity of a horizontal well incorporating the no-flow outer boundary condition are more complex than their steady-state counterparts, but generally will be more realistic and practical for a horizontal well. Babu and Odeh [24] have given a derivation of complicated analytic expressions for the pseudo-steady state productivity of a horizontal well in a finite box-shaped drainage volume of a 3D-reservoir. The well is assumed to have finite length at an arbitrary location, with alignment along the y-axis of the box. Some slightly simplified approximations for these solutions are then illustrated by these authors in a summary paper [25]. A simpler analytic solution for the pseudo-steady state productivity of a horizontal well, has been derived by Goode and Kuchuck [26], which includes anisotropy in $k_{x}, k_{y}, k_{z}$. They have considered a horizontal well parallel to the $\mathrm{x}$ direction producing from a rectangular region of dimensions $x_{e} \times y_{e}$ with uniform thickness. The well of length $L$ is located at $x_{w}, y_{w}$ and $z_{w}$. Under the reasonable assumption that the thickness of the reservoir is small compared with the distance from the well to any of the boundaries in the $\mathrm{x}$ or $\mathrm{y}$ direction, the well is treated as an infinitely conductive fracture which fully penetrates the formation. The partial penetration in the $\mathrm{z}$ direction is accounted 

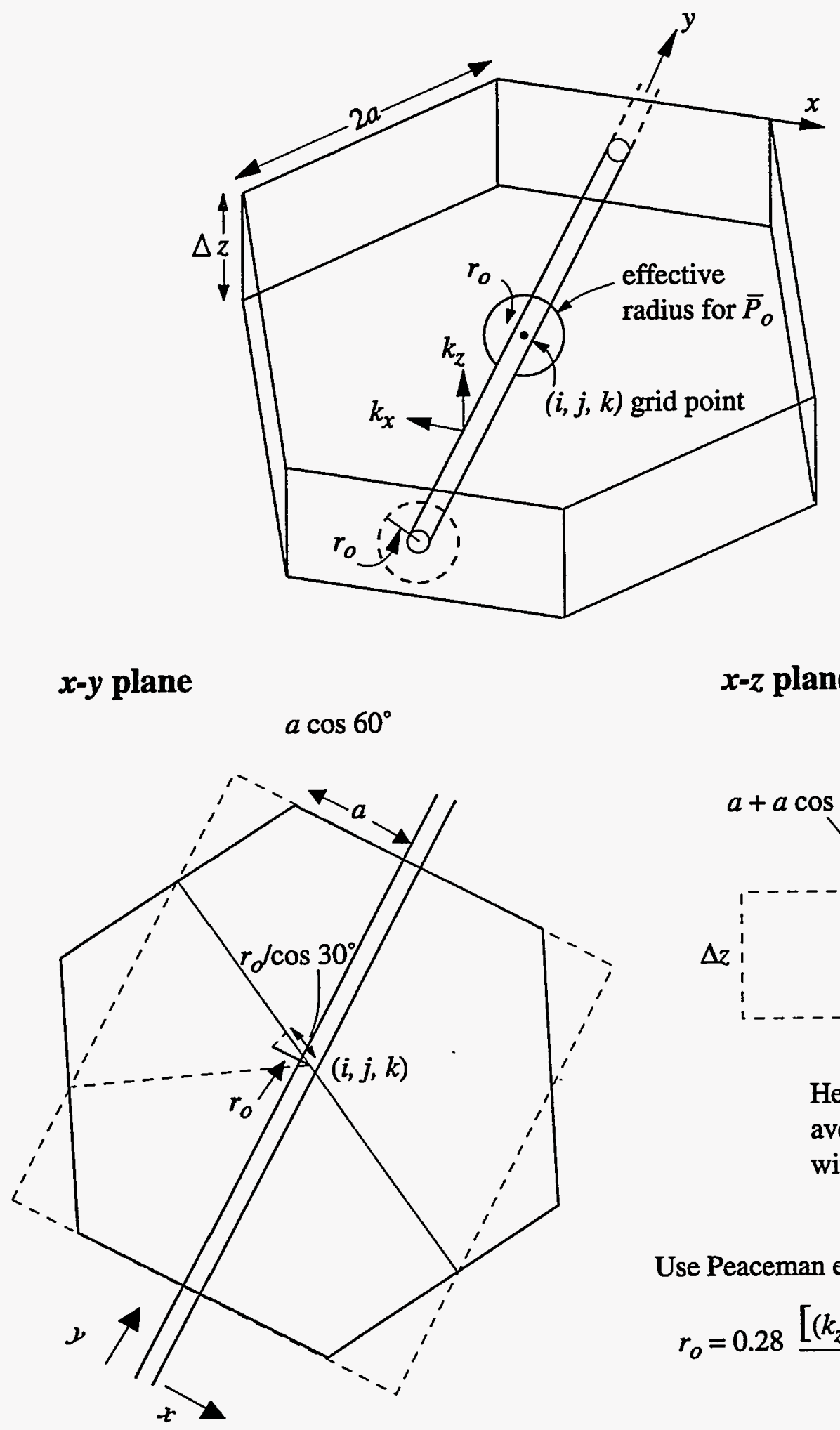

$x-z$ plane

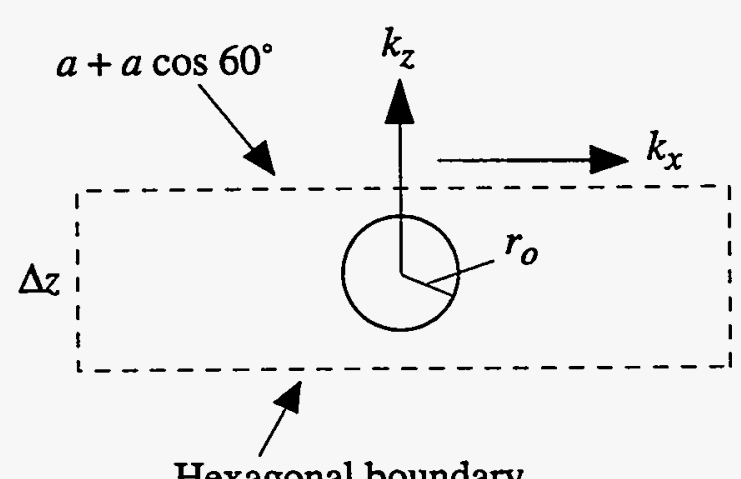

Hexagonal boundary averaged into rectangle with $\Delta x=2 a\left(1+\cos 60^{\circ}\right)$

Use Peaceman equation

$$
r_{o}=0.28 \frac{\left[\left(k_{z} / k_{x}\right)^{1 / 2} \Delta x^{2}+\left(k_{x} / k_{z}\right)^{1 / 2} \Delta y^{2}\right]}{\left(k_{z} / k_{x}\right)^{1 / 4}+\left(k_{x} / k_{z}\right)^{1 / 4}}
$$

Figure 3.6: 3-D Hexagonal Grid and its Equivalencing for Calculating $r_{o}$. 
for by a geometric skin factor. The dimensionless pressure is derived as [26]

$$
\begin{aligned}
P_{w D}= & \frac{2 \pi y_{e}}{y_{w}} \sqrt{\frac{k_{x}}{k_{y}}}\left(\frac{1}{3}-\frac{y_{w}}{y_{e}}+\frac{y_{w}^{2}}{y_{e}^{2}}\right) \\
& +\frac{8 x_{e}^{2}}{\pi^{2} L^{2}} \sum_{n=1}^{\infty} \frac{1}{n} E_{x}^{2}(1+\xi)+S_{z D}
\end{aligned}
$$

where $S_{z D}$ is the dimensionless skin factor given by

$$
\begin{aligned}
S_{z D}= & -\frac{h}{L} \sqrt{\frac{k_{x}}{k_{z}}}\left\{\ln \left[\frac{2 \pi \bar{r}_{w}}{h} \sin \left(\frac{\pi z_{w}}{h}\right)\right]\right. \\
& \left.+\sqrt{\frac{k_{x}}{k_{z}}} \frac{2 h}{L}\left(\frac{1}{3}-\frac{z_{w}}{h}+\frac{z_{w}^{2}}{h^{2}}\right)\right\}
\end{aligned}
$$

where

$$
\begin{aligned}
\bar{r}_{w} & =\left(r_{w} / 2\right)\left(1+\sqrt{k_{z} / k_{y}}\right) \\
\xi & =\frac{2 e^{\alpha y_{e}}+e^{\alpha\left(y_{e}-y_{w}\right)}+e^{\alpha y_{w}}}{1-e^{\alpha y_{e}}} \\
\alpha & =-\frac{2 n \pi}{x_{e}} \sqrt{\frac{k_{x}}{k_{y}}}
\end{aligned}
$$

and

$$
E_{x}=\frac{1}{n} \sin \left(n \pi \frac{L}{2 x_{e}}\right) \cos \left(n \pi \frac{x_{w}}{x_{e}}\right)
$$

The expression for the geometric skin, $S_{z D}$, given in Eq. (3.23) is approximate and is valid for $\sqrt{k_{x} / k_{z}}(h / L)<2.5$. Although this condition is almost always satisfied, the full expression for calculating $S_{z D}$ is given by Eq. (A-21) in Ref. [26]. The productivity index (PI) can then be calculated from

$$
P I=\frac{2 \pi \sqrt{k_{x} k_{y}} h}{\mu_{o} B_{o}\left(P_{w D}+S_{m}^{\star}\right)}
$$

in which

$$
S_{m}^{\star}=(h / L) \sqrt{k_{x} / k_{z}} S_{m}
$$

and $S_{m}$ is the mechanical skin.

We have used the above method to compute PIs for a numerical example. A reservoir with dimensions of $x_{e}=5000, y_{e}=5500, h=120 \mathrm{ft}$ with $\mu_{0}=2 c P$ and $B_{0}=$ $1.22 \mathrm{RB} / \mathrm{STB}$ is being produced by a single horizontal well of length $L=4000 \mathrm{ft}$ with $r_{w}=0.26 \mathrm{ft}$ located at $x_{w}=2500, y_{w}=2750$, and $z_{w}=30 \mathrm{ft}$. Permeabilities in the $\mathrm{x}$ and y directions are taken as $k_{h}=100 \mathrm{mD}$ whereas the vertical permeability $k_{v}$ in the $z$ direction is varied. Variation of the PI with the length of the well is calculated for three values of $k_{v} / k_{h}=1.0,0.1$, and 0.01 with $S_{m}=0$. Results are shown in Fig. 3.7. It can be seen that the productivity increases almost linearly with the length of the well for a fixed ratio of $k_{v} / k_{h}$. The PI also increases, as expected, with an increase in the vertical permeability. 
The effect of a change in the wellbore radius on the PI is also studied. Results with a smaller wellbore radius of $0.18 \mathrm{ft}$ and $k_{v} / k_{h}=0.1$ are shown in Fig. 3.7 where a weak dependence of PI on the wellbore radius is obtained for this example.

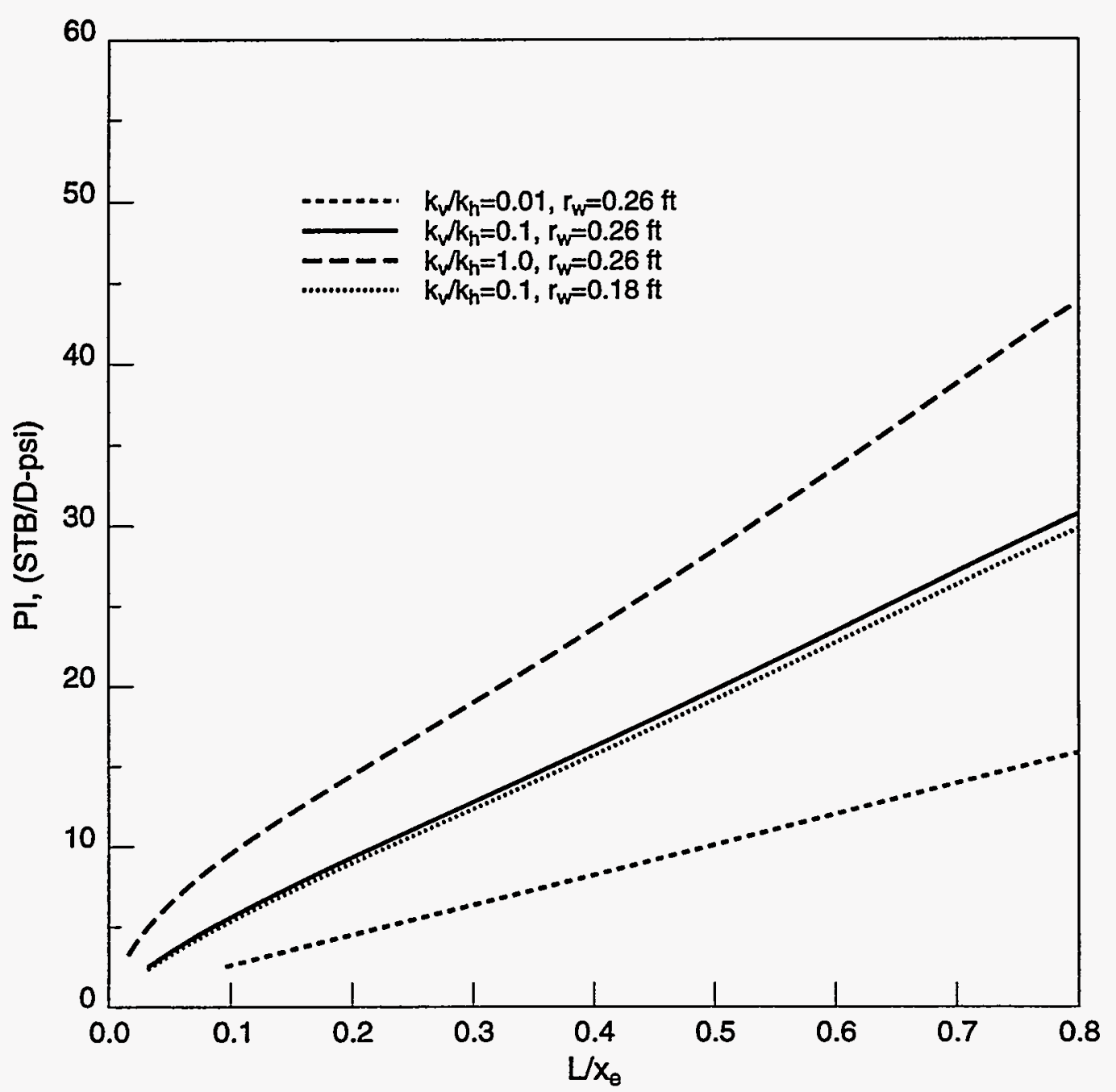

Figure 3.7: Variations of PI for the Example Problem

All the above results apply to a horizontal well which is fully open. In this case, the productive length of the well is the entire horizontal drilled section within the reservoir. However, in many practical cases depending on the completion method, the well might have several open intervals of arbitrary spatial distribution. Goode and Wilkinson [27] have developed expressions for calculating the productivity index for partially open horizontal wells. Their result is based on the method of Goode and Kuchuck [26] summarized above. In a revealing example problem, the authors have considered three ways of distributing the open intervals for a horizontal well. In the first case, the producing length of the well consists of five open segments each contributing $4 \%$ to the total length of the well. The five open 
intervals are positioned uniformly along the length of the well. The second case has two open intervals, each being $10 \%$ of the well and placed at each end of the well. In the third case there is a single open interval at the center of the well, which makes this case equivalent to a fully open well having a length one fifth of the original well. All these cases have the same fraction of total producing length of a fully open well. The results in [27] show that the first completion scenario has the highest productivity among these three cases for both thin $\left(h_{D}=\beta h / x_{e}=0.01\right)$ and thick $\left(h_{D}=0.1\right)$ reservoirs. Variation of PI with the open well fraction is of practical importance. In many situations either by choice or by accident, only a portion of the drilled well is contributing to the production. This situation is considered for the first completion case discussed above. The results indicate that in a thin reservoir with $h_{D}=0.01$, a $50 \%$ decrease in the open length of the well reduces the PI by only about $8 \%$, whereass for a thick resservoir with $h_{D}=0.1$ the same reduction in the open well fraction decreases the PI by nearly 35\% (a modest decrease). Figure 3.8 reproduced from Ref. [27] highlights these results in which the $\mathrm{Pi}$ is nomalized by that of the fully open well.

As with vertical wells, one of the difficulties with horizontal wells is to determine the appropriate skin factor. The interpretations of well tests in terms of a quantitative skin can be non-unique due to uncertainity in $k_{v} / k_{h}$. Since horizontal wells are much longer than their vertical counterparts, the skin factor is unlikely to be constant along the length. There is a tendency for the initial well damage to be larger near the heel of the well, rather than at the toe, and this can sometimes overcompensate the effects of the larger drawdown near the heel of the well. There are opportunities to vary the perforation density or completion procedure to minimize the tendencies for a gas or water crest to break through along a given section of a horizontal well.

The productivity of horizontal wells can be stimulated by hydraulic fracturing or by acidizing. It is only fair to compare horizontal and vertical well performance on the basis of their having received comparable levels of stimulation*. For horizontal wells, the opportunity to fracture parallel to their length, as opposed to transversely (see Figs. 2.6a and 2.6b), has attractions. The total length of the parallel fracture is now in contact with the well giving a linear pressure drop equation, instead of contact just around the well circumference with a logarithmic pressure drop for the transverse fracture. The latter can cause significant choking if the fracture flow is large. However, the parallel fracture orientation advantage can be offset in low permeability reservoirs by the geometry favoring stimulation of a number of transverse fractures. Multiple fractures may be needed to give useful enhancement of productivity, but this then increases the workover costs. There may be factors in the depositional environment, other than the natural fractures or principal stress direction, which on balance influence the best orientation for a horizontal well. It has also been found [28] that despite choosing the well axis along the minimum stress direction, that the perturbation induced in the local stress field can cause a fracture to strike off parallel to the well, before twisting into the orthogonal direction.

*This may depend on the costs of workover of horizontal wells being brought into line with such costs for vertical wells. 


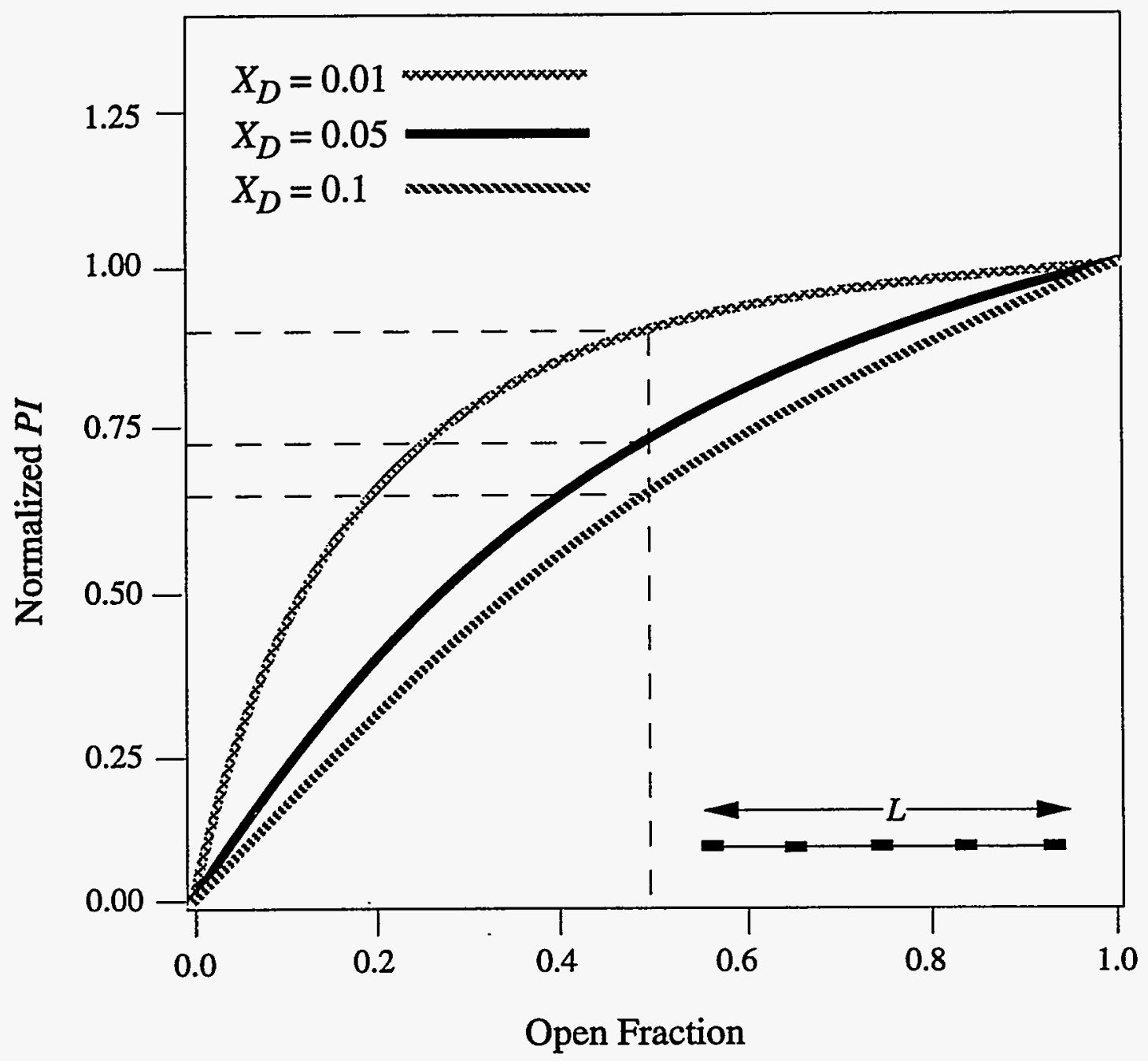

Figure 3.8: Variations of PI as a Function of Open Well Fraction [27] 


\subsection{General Permeability Anisotropy}

The existence of anisotropy in permeability can cause special problems when the well is not aligned along one of the principal axes. In the general case the well direction can be defined by two polar angles $(\omega, \theta)$ relative to the $\mathrm{x}$ and $\mathrm{z}$-axes respectively, which we assume coincide with the principal permeability directions. The anisotropic Laplace equation

$$
k_{x} \frac{\partial^{2} P}{\partial x^{2}}+k_{y} \frac{\partial^{2} P}{\partial y^{2}}+k_{z} \frac{\partial^{2} P}{\partial z^{2}}=0
$$

scales to

$$
\bar{k}\left(\frac{\partial^{2} P}{\partial \bar{x}^{2}}+\frac{\partial^{2} P}{\partial \bar{y}^{2}}+\frac{\partial^{2} P}{\partial \bar{z}^{2}}\right)=0
$$

if the coordinates are scaled by the transformations $\bar{x}=\left(\bar{k} / k_{x}\right)^{1 / 2} x, \bar{y}=\left(\bar{k} / k_{y}\right)^{1 / 2} y$, and $\bar{z}=\left(\bar{k} / k_{z}\right)^{1 / 2} z$. In three dimensions

$$
\bar{k}=\left(k_{x} k_{y} k_{z}\right)^{1 / 3}
$$

while in two dimensions with an infinite well in the $y$-direction

$$
\bar{k}=\left(k_{x} k_{z}\right)^{1 / 2}
$$

The Peaceman results for Cartesian $(x z)$ grid systems can be expressed in terms of $\bar{k}$ as: the transformed grid radius

$$
\begin{aligned}
\bar{r}_{o y} & =0.14\left(\triangle \bar{x}^{2}+\triangle \bar{z}^{2}\right)^{1 / 2} \\
& =0.14 \bar{k}^{1 / 2}\left(\frac{\triangle x^{2}}{k_{x}}+\frac{\triangle z^{2}}{k_{z}}\right)^{1 / 2}
\end{aligned}
$$

and the transformed well radius

$$
\bar{r}_{w y}=\frac{1}{2} r_{w} \bar{k}^{1 / 2}\left(\frac{1}{\sqrt{k_{x}}}+\frac{1}{\sqrt{k_{z}}}\right)
$$

Then

$$
\begin{aligned}
r_{o y} & =\frac{r_{w}}{\bar{r}_{w y}} \bar{r}_{o y} \\
& =0.28 \frac{\left(\frac{\Delta x^{2}}{k_{x}}+\frac{\Delta z^{2}}{k_{z}}\right)^{1 / 2}}{\left(\frac{1}{\sqrt{k_{x}}}+\frac{1}{\sqrt{k_{z}}}\right)}
\end{aligned}
$$

Equation (3.34) is the Peaceman equation (3.2) and is independent of the definition of $\bar{k}$. Similar results also define $r_{o x}$ and $r_{o z}$ if the well were aligned with the $\mathrm{x}$ or $\mathrm{z}$-coordinate directions. A reasonable equation for the general case of a well orientation defined by $(\omega, \theta)$ is given by

$$
r_{o}=\left[r_{o x}^{2}(\sin \theta \cos \omega)^{2}+r_{o y}^{2}(\sin \theta \sin \omega)^{2}+r_{o z}^{2} \cos ^{2} \theta\right]^{1 / 2}
$$

Equation (3.35) defines an ellipsoid such that each of the limiting cases of the well aligned in $\mathrm{x}, \mathrm{y}$, or z-directions are satisfied, and otherwise the effective $r_{o}$ is weighted by the direction cosines of the well with respect to these directions. Generalization of this treatment to Voronoi grids does not appear to have been attempted. 


\subsection{Averaging Procedures for Heterogeneities}

The Voronoi grid, or any other general gridding scheme, encounters difficulties once reservoir heterogeneities are considered. The Voronoi grid shown in Fig. 3.9 has a pattern of heterogeneities.imposed upon it. The pattern assumed is relatively coarse compared with what it is now possible to generate using modern geostatistical techniques. Nevertheless, it is still necessary to pose the question of the averaging algorithms which will correctly determine the effective permeability for each direction of flow across the cell faces. In general the problem will have a tensor characteristic, so that in a Cartesian system the flux in the $x$-direction takes the form

$$
q_{x}=\frac{1}{\mu}\left(k_{x x} \frac{\partial P}{\partial x}+k_{x y} \frac{\partial P}{\partial y}+k_{x z} \frac{\partial P}{\partial z}\right)
$$

where $k_{x y}, k_{x z}$ are off-diagonal elements for the $x$-direction of a $3 \times 3$ tensor (i.e. the $y$ and $z$-components of pressure gradient influence the $x$-direction flux).

Simple permeability averaging theorems to handle this aspect of fluid flow in a heterogeneous system have been proposed by Palagi [18]. The procedure given below is an adaptation of Palagi's approach, extended for horizontal wells in an anisotropic reservoir. The analysis described in Section 3.2 for calculating $r_{o}$, which depended on taking differences between an analytic solution and a coarse grid solution, can be extended to heterogeneous problems. In this case the analytic solution is replaced by a fine grid numerical solution for a model problem which contains the details of the heterogeneity. The fine grid needs to be chosen such that individual blocks near the wells each have "homogeneous" properties. Some compromises are needed here, since it is known that small Cartesian blocks can give significant errors [18] if $\Delta z \ll \Delta x$ which is the natural choice for a horizontal well along the y-direction. In principle, the coarse grid model can be solved using an average $\bar{k}_{\text {con }}$ for the connection between the well and $r_{o}$, and also $\bar{k}_{i j}$ for the average permeability between a grid point $i$ and grid point $j$. An approximate method for averaging heterogeneities based on a grid of permeability values within the area shown in Fig. 3.10 can be used, in which the anisotropy is constrained to $\mathrm{x}$ and $\mathrm{z}$-directions in the form:

$$
\bar{k}_{x i j}=\left[\sum_{l} \sum_{m}\left(k_{x l m}^{\omega_{x}}\right)\right]^{1 / \omega_{x}}
$$

Similarly when the (ij)-connection is in the z-direction,

$$
\bar{k}_{z i j}=\left[\sum_{p} \sum_{q}\left(k_{z p q}^{\omega_{z}}\right)\right]^{1 / \omega_{z}}
$$

The parameters $\omega_{x}, \omega_{z}, \bar{k}_{\text {con }}, r_{o}$ can be considered as history matching quantities for minimization of the differences in solution between the fine grid and coarse grid model problem. Palagi found the value $\omega_{x} \approx-0.5$ fitted his results. Provided the heterogeneities are not too severe, then this procedure can be satisfactory, and could be extended to three dimensions. However, it will break down when strongly correlated permeability distributions 
(mD)

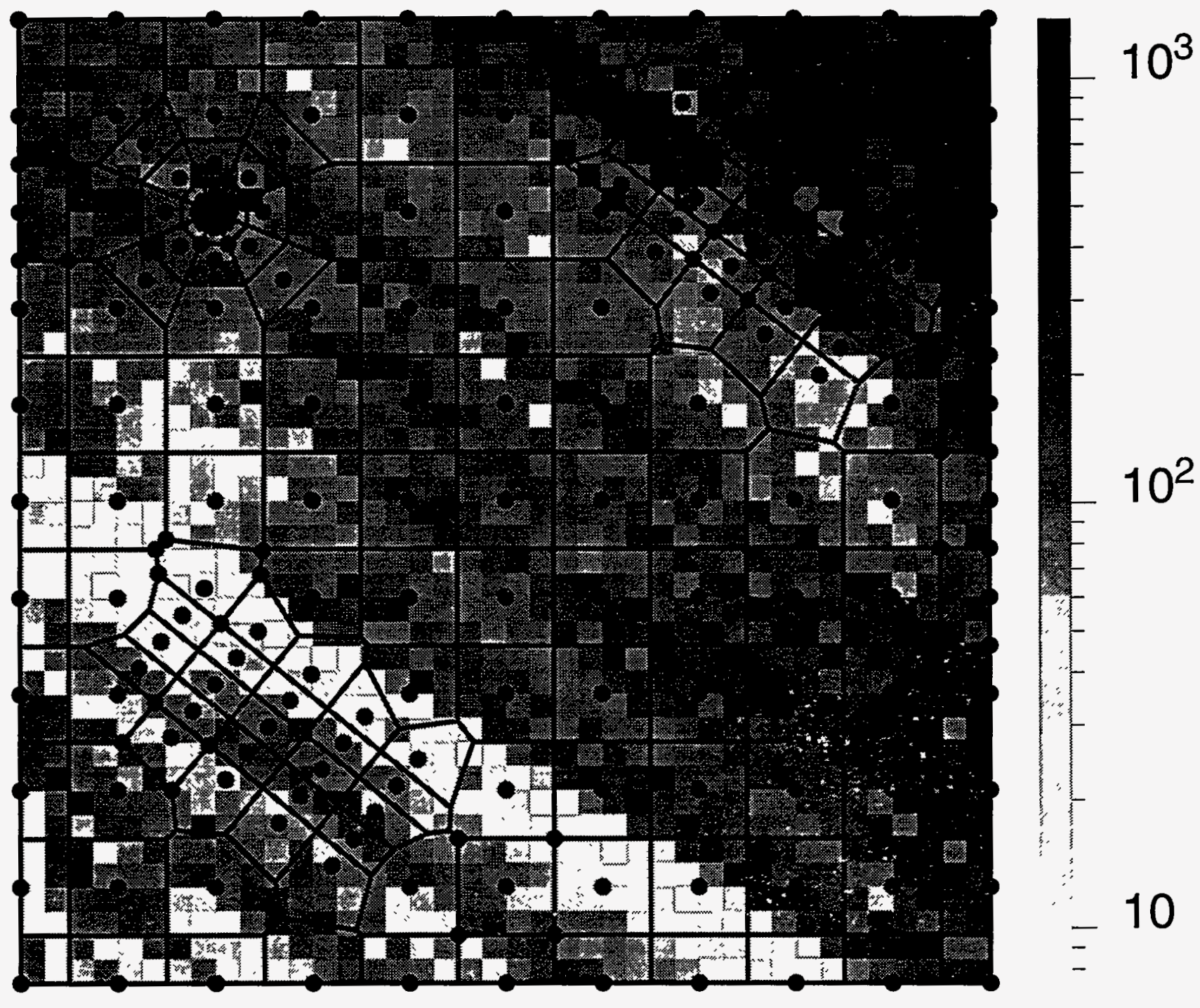

Figure 3.9: Averaging Problems Associated with Introduction of Heterogeneities into a Generalized Grid 


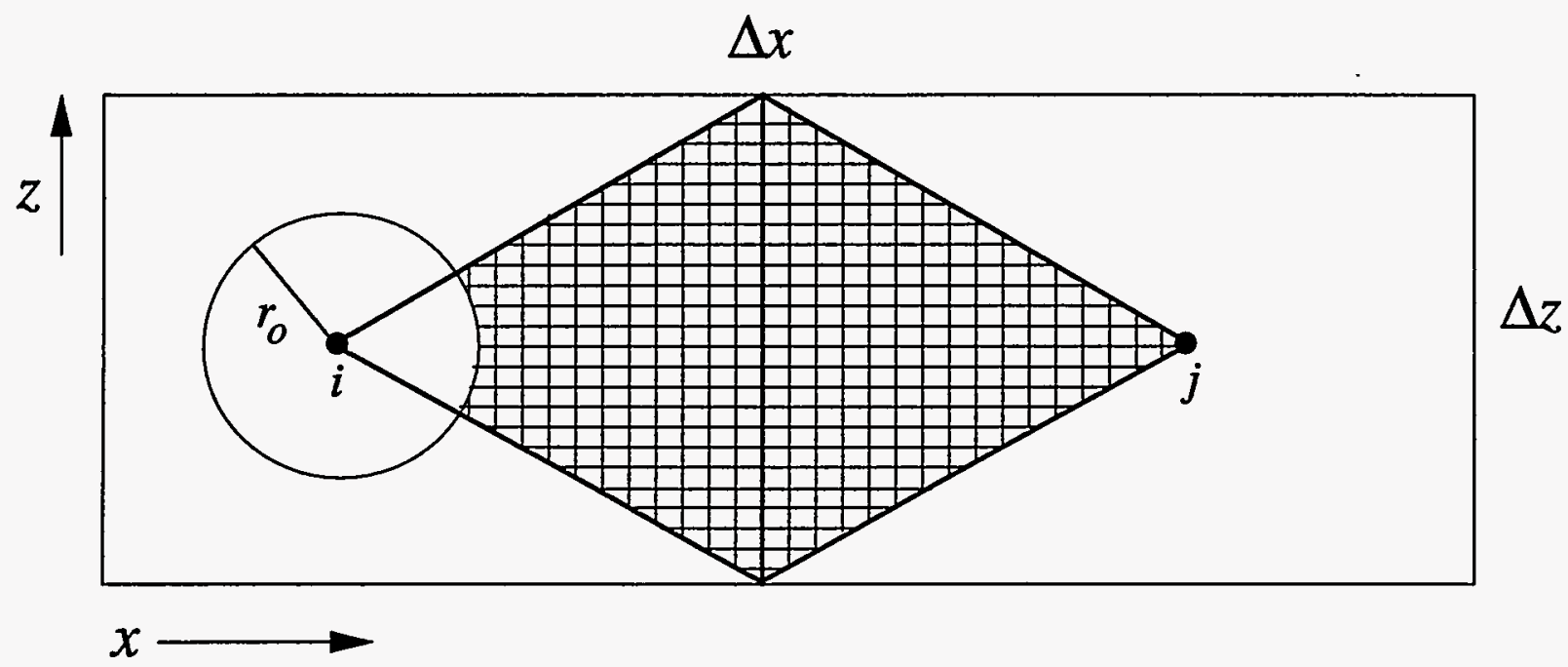

Figure 3.10: Permeability Averaging Area for Connection Between Well Block $i$ and its Neighbor $j$

are involved. The problem also becomes more severe when triangular or hexagonal cells are to be considered where boundaries do not conform to principal permeability directions. The horizontal well imposes its own special difficulties since the flow is essentially radial in a plane where $k_{v} \ll k_{h}$ may apply; i.e., the flow is inevitably not aligned with any principal directions for permeability anisotropy. When two-phase flow is to be computed, the effective relative permeabilities will also have directional characteristics. Gravity now influences the fractional flow in a non-radial sense. The averaging procedures for cells adjacent to a horizontal well need to have a special formulation which should take cognizance of the nearly normal flow at the well circumference.

Examples of sensitivity of two-phase behavior to gridding arrangements in a heterogeneous reservoir have been presented by Consonni et al. [29]. The horizontal well part of their studies was concerned with a 3D aquifer coning problem for a production well placed near to the top of the oil column. Eclipse calculations using some 35,000 Cartesian grid blocks were regarded as accurate solutions for this problem (although even this may not be completely satisfactory). Fig. 3.11 shows parallel and diagonal coarse grid alternatives for representing the reservoir, with a Voronoi fine grid introduced close to the well. Fig. 3.12 gives the water-cut histories associated with these two gridding arrangements for a reservoir description with correlated heterogeneities. The divergence of the results is significant, for reasons that are not clearly understood. For the equivalent parallel and diagonal grids in the homogeneous case, there is quite close agreement between the predicted water cut behaviors. 


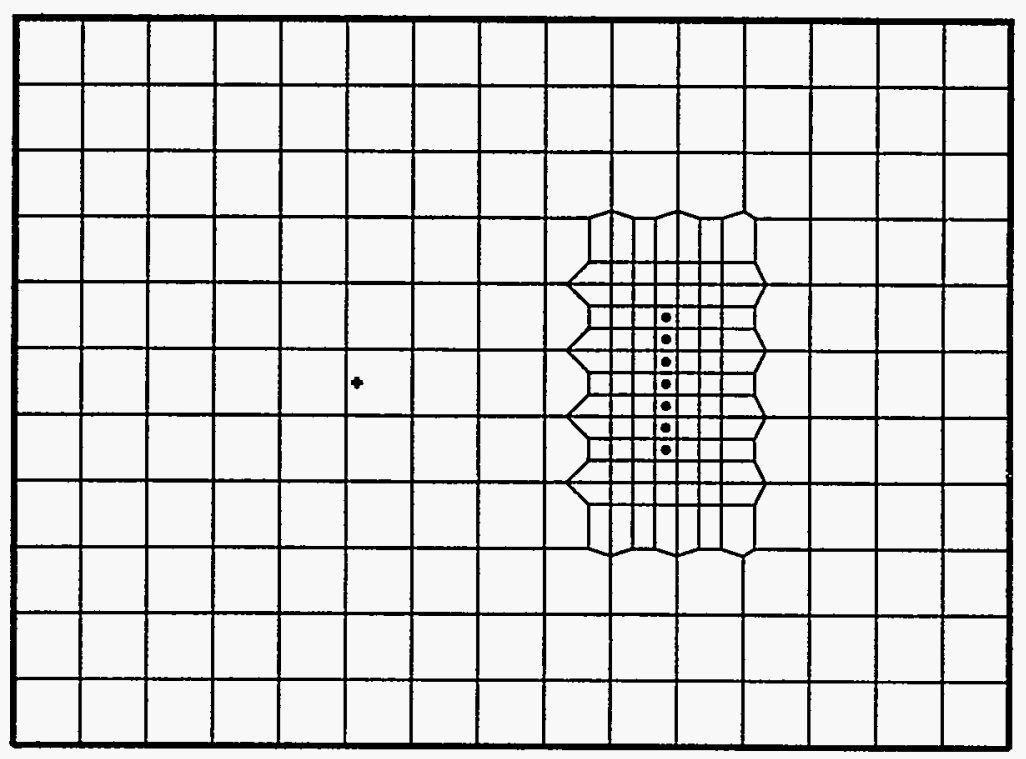

Grid A

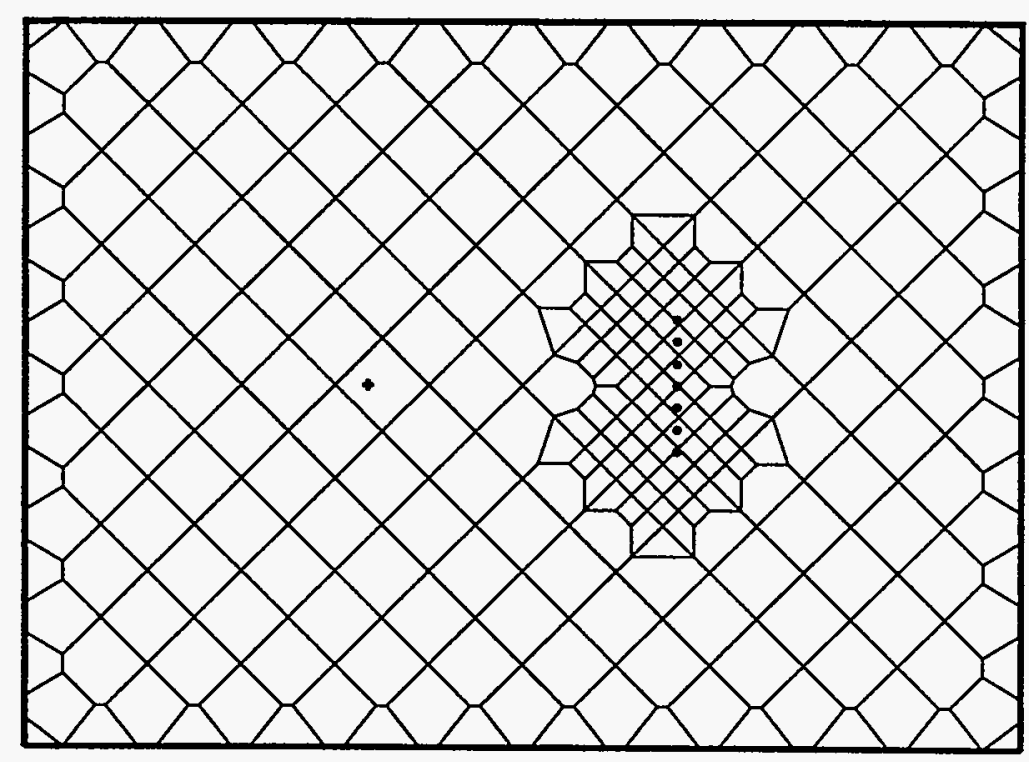

\section{Grid B}

Figure 3.11: Parallel and Diagonal Voronoi Grids A and B [29] 


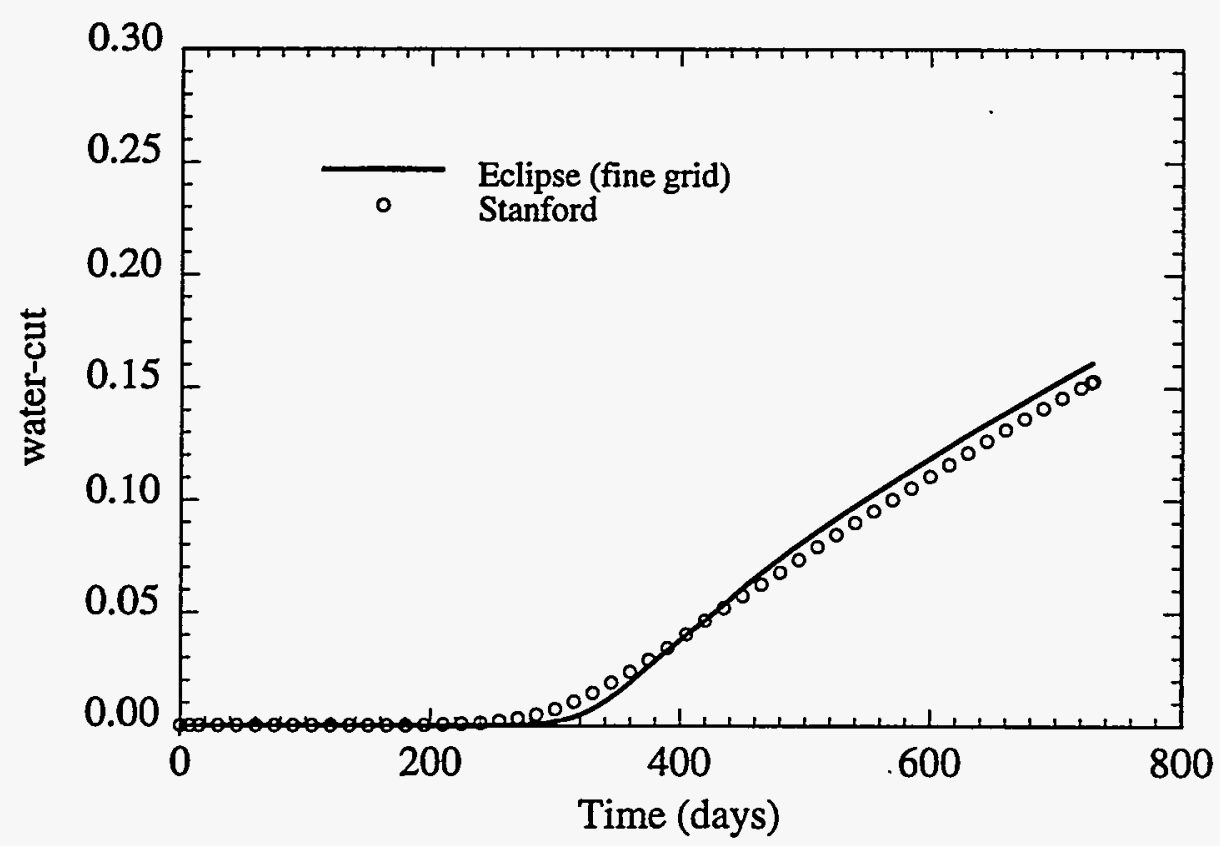

Grid A

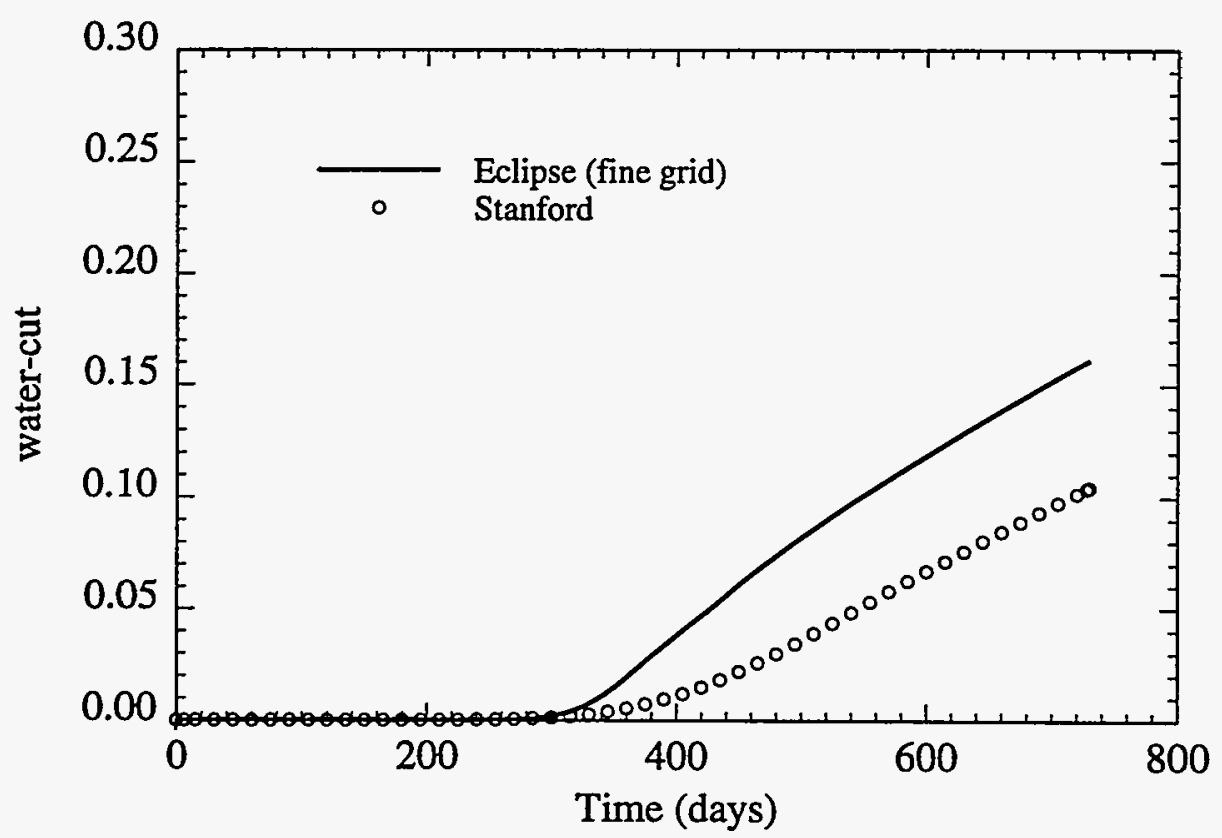

Grid B

Figure 3.12: Water Cut History for Voronoi Grids A and B [29] 


\section{End Effects On Productivity Index Of Horizontal Wells (Task 1)}

This study has been conducted by research assistant Obaid Al-Harthy, together with Profs. Roland N. Horne and F. John Fayers, as part of an M.S. research project.

\subsection{Introduction}

Most horizontal well Productivity Index (PI) expressions currently in use do not account for the effects of the ends of the well where flow is spherical. Usually, flow is assumed radial throughout the well and a single value of well PI is assigned for the entire well. In this study, we develop a horizontal well PI expression that accounts for the end effects. The expression was developed for a truncated line source using the method of Green's functions. In our derivation, we have assumed a uniform flux boundary condition along the well. We have shown that the horizontal well PI is a function of both position and time.

We have also explored the possibility of incorporating our results in reservoir simulations. We compared our analytical results, with those of the Eclipse simulator, which uses a single value of well PI for all positions and times. The analytical model gives a PI which varies with position along the well and also varies with time, At the center of the well and at large times, the analytical PI values are in agreement with the Eclipse value. However, at the end of the well or at early time, the analytical PI values are much higher than the Eclipse value.

\subsection{Mathematical Expression for PI}

For convenience, we have used the truncated line source solution for this study. We also used the infinite line source solution for checking purposes. The well Productivity Index (PI) is defined as the ratio of the flow rate to the pressure drawdown. Using the results to be published in a separate report, we obtain a PI expression:

$$
\begin{aligned}
P I & =\frac{q}{\Delta p} \\
& =\frac{2 \pi k L}{\mu p_{D}} \\
& =\frac{8 \pi k L}{\mu S} \\
\text { where } S= & \int_{0}^{t} \frac{1}{t} \exp \left[-\frac{\left(x^{2}+z^{2}\right)}{4 \eta t}\right] \\
& {\left[\operatorname{erf}\left\{\frac{y+L / 2}{\sqrt{4 \eta t}}\right\}-\operatorname{erf}\left\{\frac{y-L / 2}{\sqrt{4 \eta t}}\right\}\right] d t }
\end{aligned}
$$




\subsubsection{Variation of PI Along the Well}

The dimensionless pressure drop, shown in the previous section, is inversely proportional to the well PI. We define a dimensionless productivity index $P I_{D}$ :

$$
P I_{D}=\frac{\mu}{2 \pi k L} P I \Rightarrow P I_{D}=\frac{1}{p_{D}}
$$

A computer program was written to evaluate $P I_{D}$ as a function of position along the well. $p_{D}$ in Eq. (4.2) was solved numerically using Simpson's rule. Figs. 4.1 and 4.2 show the variation of $P I_{D}$ as a function of both position $L_{D}$ and dimensionless time $t_{D}=0.000264 k t / \phi \mu c_{t} r_{w}^{2}$.

\subsubsection{Well PI for Use in Reservoir Simulators}

Since the pressure drop is not uniform along the well, we evaluate a different PI for each block in which a well is completed. The PI for block $(i, j, k)$ is given by:

$$
P I_{i, j, k}=\frac{q_{i, j, k}}{p_{i, j, k}-p_{w f}}
$$

As discussed by Peaceman [30], the average block pressure is not equal to the block pressure for a block containing the well. We therefore, follow the procedure outlined by Aziz [31] to obtain $p_{i, j, k}$. If we assume incompressible and steady state flow, the flow rate into a well block is then equivalent to the sum of the flow rates from the blocks adjacent to it. For a well in the $y$ direction, the flow rate into block $(i, j, k)$ is given by:

$$
q_{i, j, k}=q_{i-1, j, k}+q_{i+1, j, k}+q_{i, j, k-1}+q_{i, j, k+1}
$$

For uniform Cartesian square grid, the well block pressure is calculated from a finite difference equation

$$
q_{i, j, k}=\frac{k \Delta y \Delta z}{\mu \Delta x}\left(p_{i-1, j, k}-2 p_{i, j, k}+p_{i+1, j, k}\right)+\frac{k \Delta x \Delta y}{\mu \Delta z}\left(p_{i, j, k-1}-2 p_{i, j, k}+p_{i, j, k+1}\right)
$$

The block flow rate in Eq. (4.5) is known for a well operating under constant rate constraint and the block pressures are obtained from the pressure equation. For example, for block $(\mathrm{i}-1, \mathrm{j}, \mathrm{k})$ the pressure is:

$$
\begin{aligned}
p_{i-1, j, k}= & p_{i}-\frac{q \mu}{8 \pi k L} \int_{0}^{t} \frac{1}{t} \exp \left[-\frac{\left(x^{2}+z^{2}\right)}{4 \eta t}\right] \\
& {\left[\operatorname{erf}\left\{\frac{y+L / 2}{\sqrt{4 \eta t}}\right\}-\operatorname{erf}\left\{\frac{y-L / 2}{\sqrt{4 \eta t}}\right\}\right] d t }
\end{aligned}
$$

The $x, y$, and $z$ in Eq. (4.6) are the coordinates of the center of block (i-1,j, k), relative to the center of the well. Defining the grid aspect ratio as:

$$
\alpha=\frac{\Delta z}{\Delta x}
$$


Table 4.1: Eclipse Input Data for Productivity Calculations

\begin{tabular}{||l|c||}
\hline Permeability, $k$ & $20 \mathrm{md}$ \\
Viscosity, $\mu$ & $10 \mathrm{cp}$ \\
Wellbore Radius, $r_{w}$ & $0.5 \mathrm{ft}$ \\
Porosity, $\phi$ & 0.3 \\
Compressibility, $c$ & $1.687 \mathrm{e}-05 \mathrm{psi}^{-1}$ \\
Formation Volume Factor, $B$ & $1.01 \mathrm{rb} / \mathrm{stb}$ \\
Initial Reservoir Pressure, $p_{i}$ & $3000 \mathrm{psi}$ \\
Well Flow Rate, $q$ & $1000 \mathrm{stb} / \mathrm{d}$ \\
Well Length, $L$ & $1000 \mathrm{ft}$ \\
Number of Grid Blocks & $17 \times 32 \times 17$ \\
\hline
\end{tabular}

The well block pressure is then given by:

$$
p_{i, j, k}=\frac{1}{2}\left(\frac{\alpha}{\alpha^{2}+1}\right)\left[\alpha\left(p_{i-1, j, k}+p_{i+1, j, k}\right)+\frac{1}{\alpha}\left(p_{i, j, k-1}+p_{i, j, k+1}\right)-\frac{\mu q_{i, j, k}}{k \Delta y}\right]
$$

The same procedure used to calculate $p_{i-1, j, k}$ is used to calculate the well block flowing pressure $p_{w f}$. In this case the value of $\mathrm{x}$ is set equal to $r_{w}$ and the value of $\mathrm{z}$ is set equal to zero. The value of $y$ is the coordinate of the center of the well block

$$
\begin{aligned}
p_{w f}= & p_{i}-\frac{q \mu}{8 \pi k L} \int_{0}^{t} \frac{1}{t} \exp \left[-\frac{r_{w}^{2}}{4 \eta t}\right] \\
& {\left[\operatorname{erf}\left\{\frac{y+L / 2}{\sqrt{4 \eta t}}\right\}-\operatorname{erf}\left\{\frac{y-L / 2}{\sqrt{4 \eta t}}\right\}\right] d t }
\end{aligned}
$$

\subsection{Comparison with Simulator Results}

We compare the results obtained from the analytical solution with the results from Eclipse simulation. Table 4.1 shows a summary of data values used in the study. We split the 1000 feet long well into 20 'shorter wells' each of length 50 feet. Each 'well' is then completed in a single grid block. The grid size in the y direction is set to 50 feet so that the 'well' penetrates the whole grid block.

Eclipse was run for a simulated time of 1000 days. The size of the outer grid blocks was made large so that the boundary effects will not be felt during the simulation. For the data set, Eclipse yielded a PI value of $0.1906 \mathrm{stb} / \mathrm{d}$-psi. This value was constant for the entire timestep and for all 20 'wells'. From Eclipse, we also obtained well block pressures $p_{i, j, k}$, well flowing pressures $p_{w f-i, j, k}$, and pressures for blocks adjacent to the well blocks in the $i-1$ blocks $p_{i-1, j, k}$. These results were obtained at one day and at 1000 days. The 'wells' were reconverted back to a single well by assigning the results to the y coordinates corresponding to well block centers.

We calculated the pressures and PI using the analytical solution from Eqs. (4.8), (4.9), and (4.3) for equivalent configuration as computed using Eclipse. We also calculated 
a normalized PI defined as

$$
P I_{N}=\frac{P I_{\text {analytical }}}{P I_{\text {Eclipse }}}
$$

The results for the two methods are shown on Figs. 4.3, 4.4, 4.5, and 4.6. Fig. 4.3 compares the pressures as functions of position at a time of 1 day and Fig. 4.4 compares the pressures as functions of position at a time of 1000 days. Fig. 4.5 shows the variation of PI with position (analytical solution) as compared to a constant value determined by Eclipse. Fig. 4.6 shows the normalized PI for the two times.

The results obtained from this study generally agrees with the results obtained from Eclipse. The main difference observed is the variation of PI as a function of both position and time. While Eclipse gives a single value of PI for each well and for the entire timestep, this study has shown that the well PI is higher at the ends of the well than at the middle. with time. The higher PI at the ends of the well is due to spherical flow as opposed to radial flow at the middle. Since we assumed a constant rate inner boundary condition (uniform flux source), the effect of spherical flow is seen as a lower pressure drop at the ends of the well than at the middle. An alternative to this study would have been to consider the constant pressure inner boundary condition (infinite conductivity source). In addition, the study has shown that the well PI decreases with time, but converges towards a steady value (after 100 days for the example shown here).

The well flowing pressures determined analytically decline slower with time than those determined using Eclipse. For the period of 1000 days the well flowing pressure has dropped by approximately 170 psi compared to a drop of approximately 220 psi for Eclipse (Figs. 4.3, 4.4). The difference between the two methods is less than two percent.

The pressure of the blocks adjacent to the well blocks match closely for the two methods (Figs. 4.3, 4.4). The differences become more apparent with increase in time. However, the overall difference is less than one percent.

A somewhat larger variation in pressure is seen for the well block pressures (Figs. 4.3, 4.4). The difference between Eclipse results and the analytical results is about two percent. This error is probably due to the neglect of the accumulation term.

Eclipse calculates well PI using the Peaceman formula which is based on the infinite line source solution. The PI value of 0.1906 predicted by Eclipse is equivalent to the PI value we obtained using the infinite line source solution with the time equal to one day.

A comparison of normalized PI $\left(P I_{N}\right)$ shows that $P I_{N}$ converges to one at the middle of the well for large times. In other words, at large times, the analytical PI approaches the Peaceman value at the middle of the well but not at the ends. Moreover, the analytical PI is always higher than the Peaceman value for all times, and more so at early times. At the center of the well and at large times, the difference between analytical PI and Peaceman PI is about six percent. However at a distance of about 150 feet from the end of the well, the error in PI starts to rise from 6 percent to about 23 percent at the ends. 


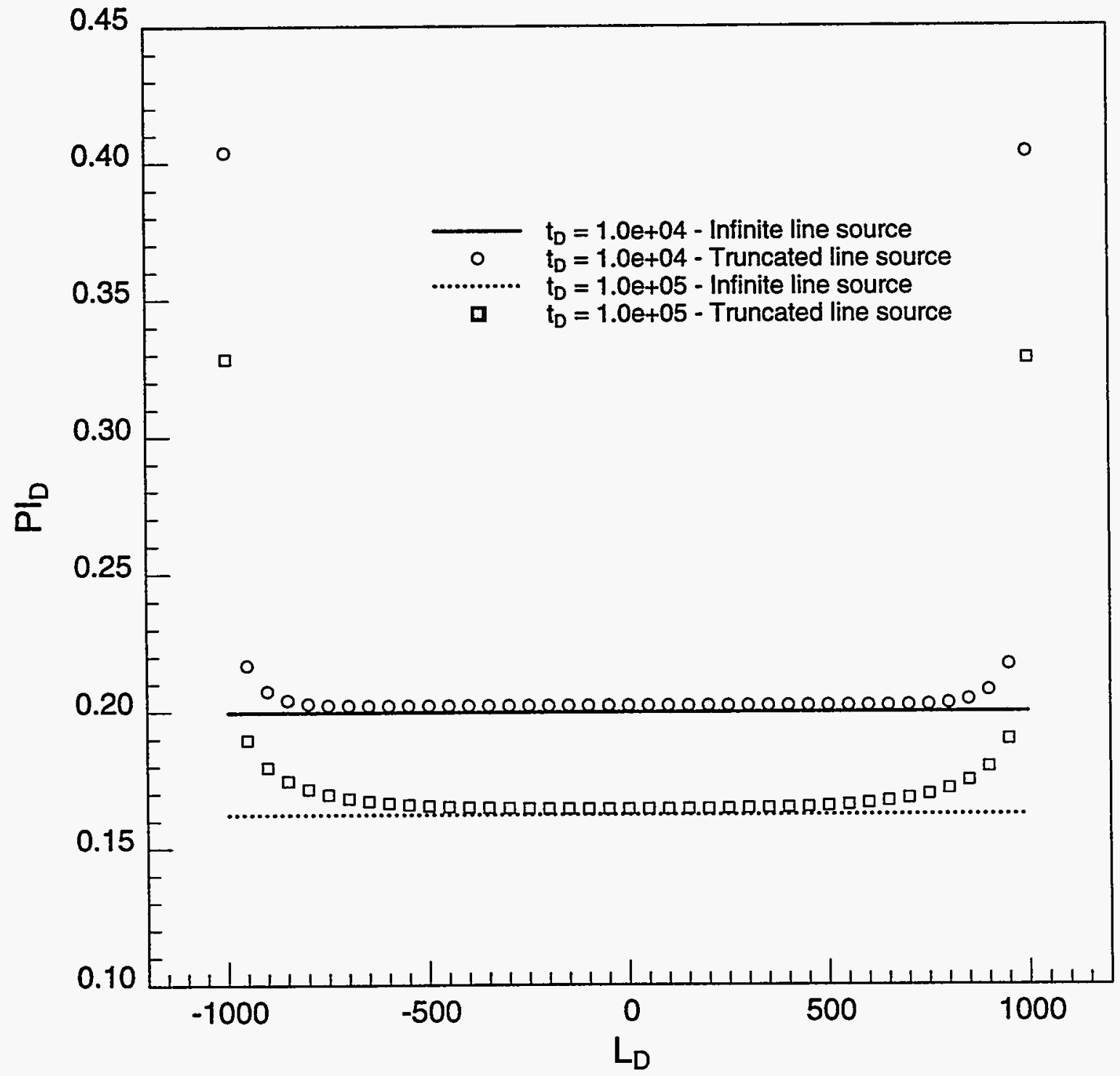

Figure 4.1: Variation of $P I_{D}$ with $L_{D}$ and $t_{D}$ 


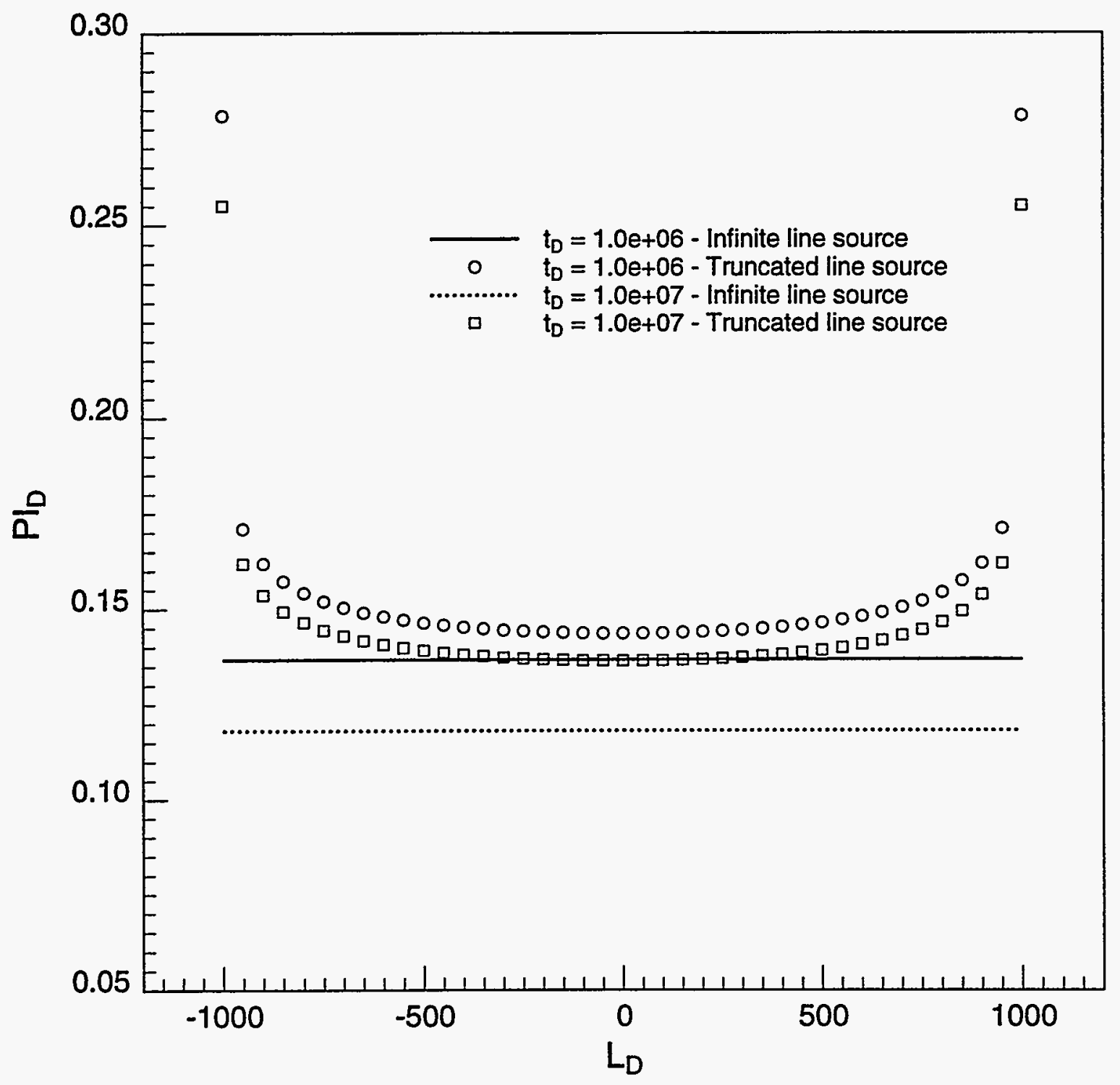

Figure 4.2: Variation of $P I_{D}$ with $L_{D}$ and $t_{D}$ 


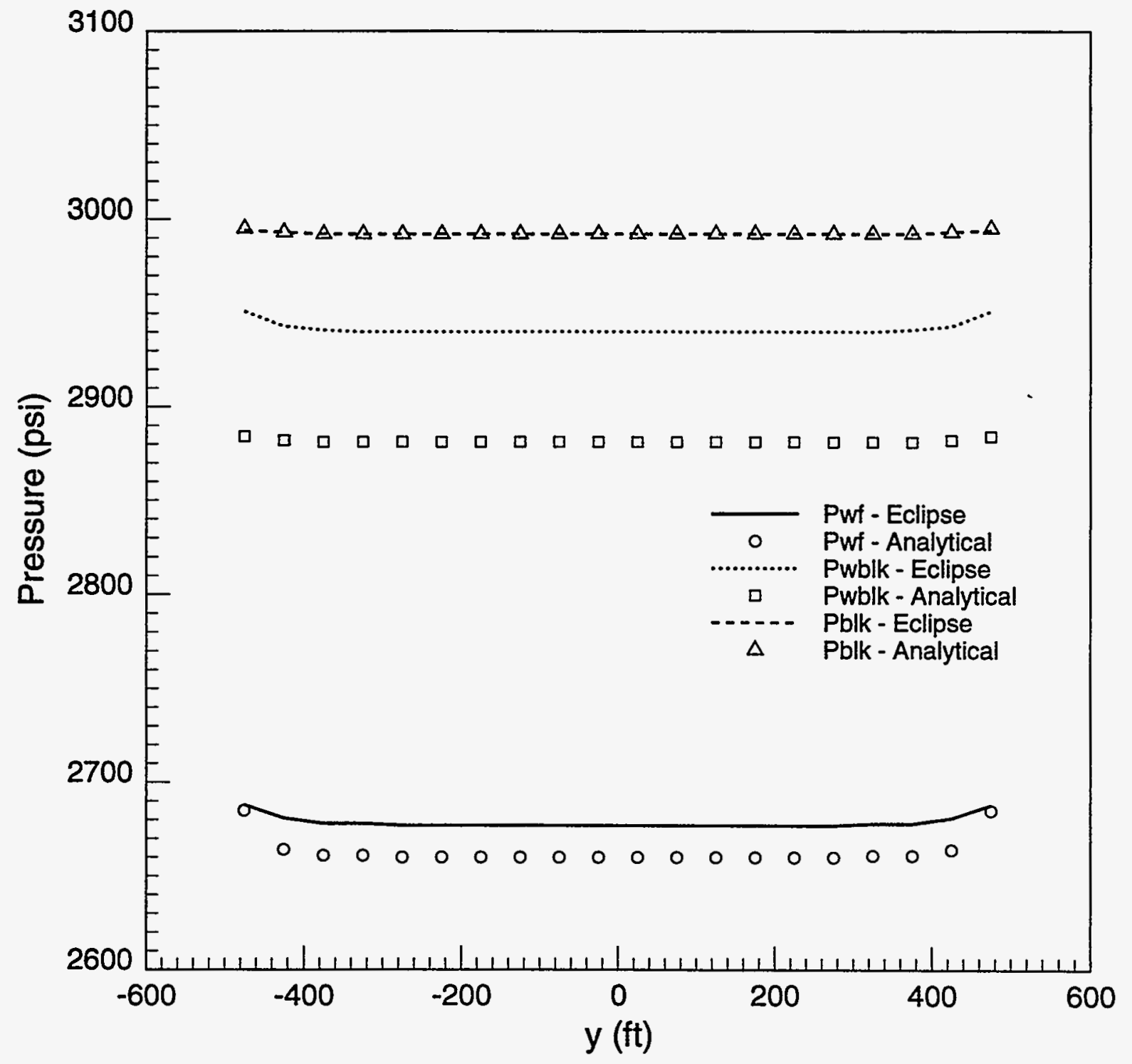

Figure 4.3: Comparison of Pressure at $t=1$ Day 


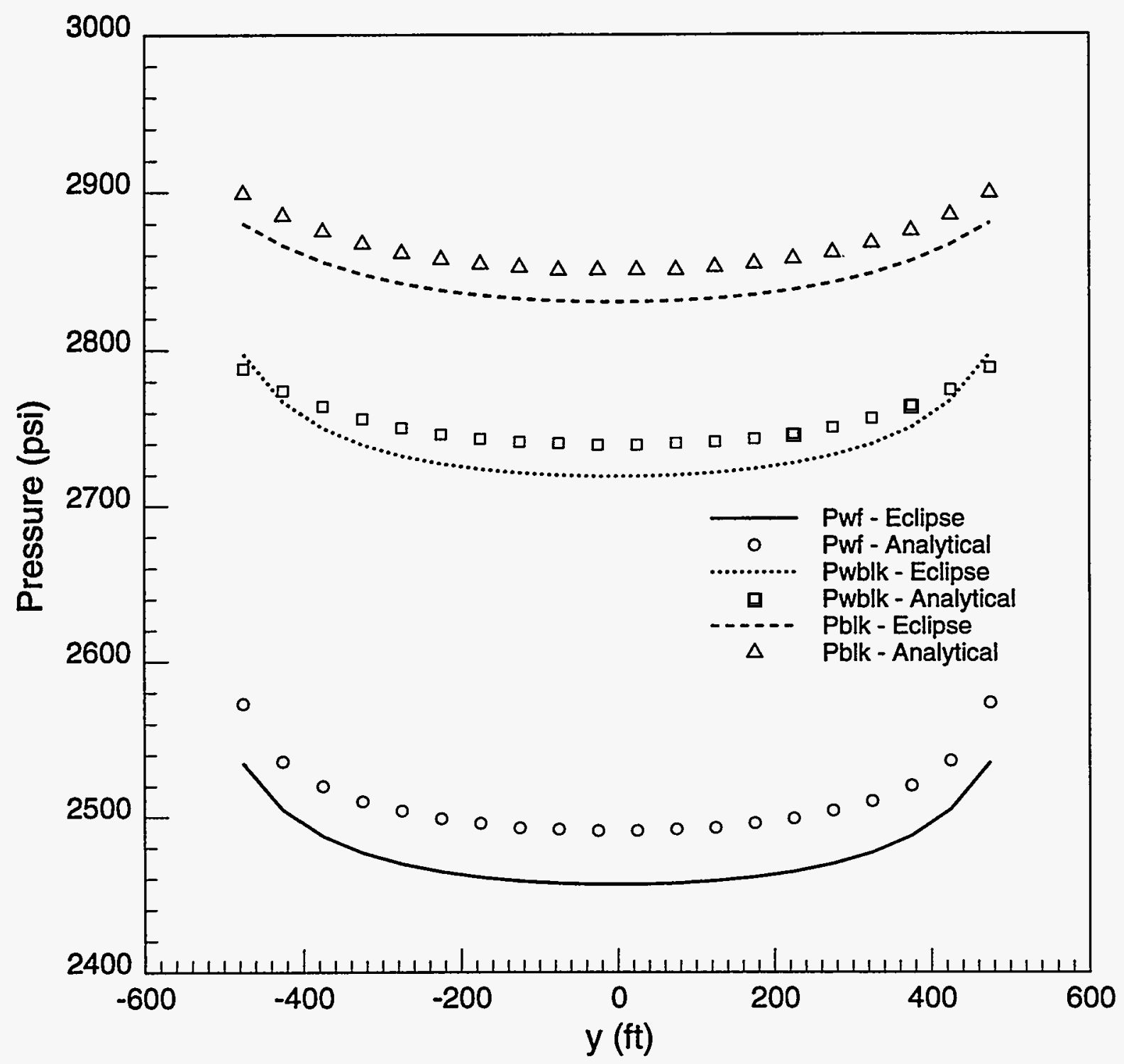

Figure 4.4: Comparison of Pressure at $t=1000$ Days 


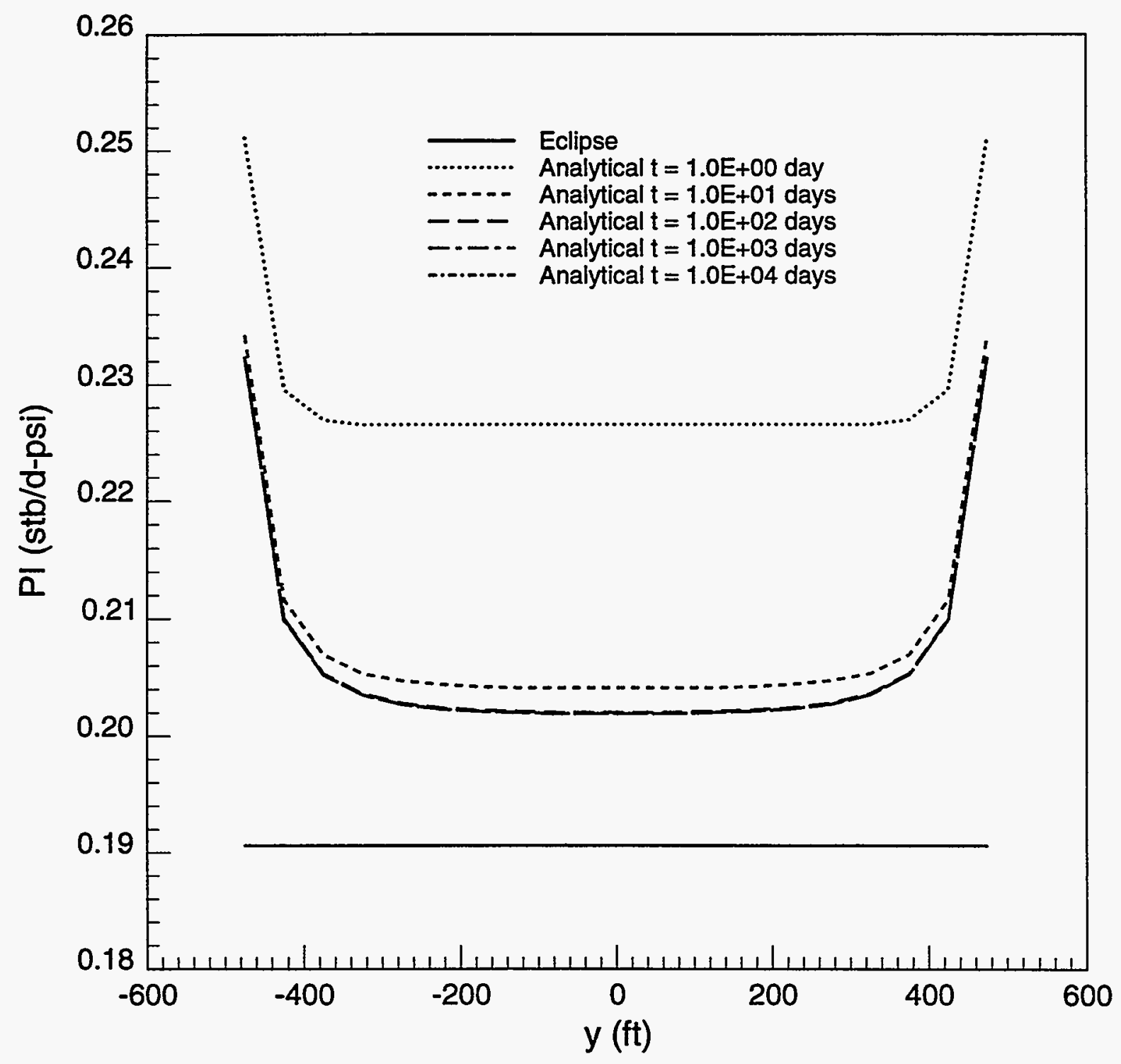

Figure 4.5: Comparison of Productivity Index 


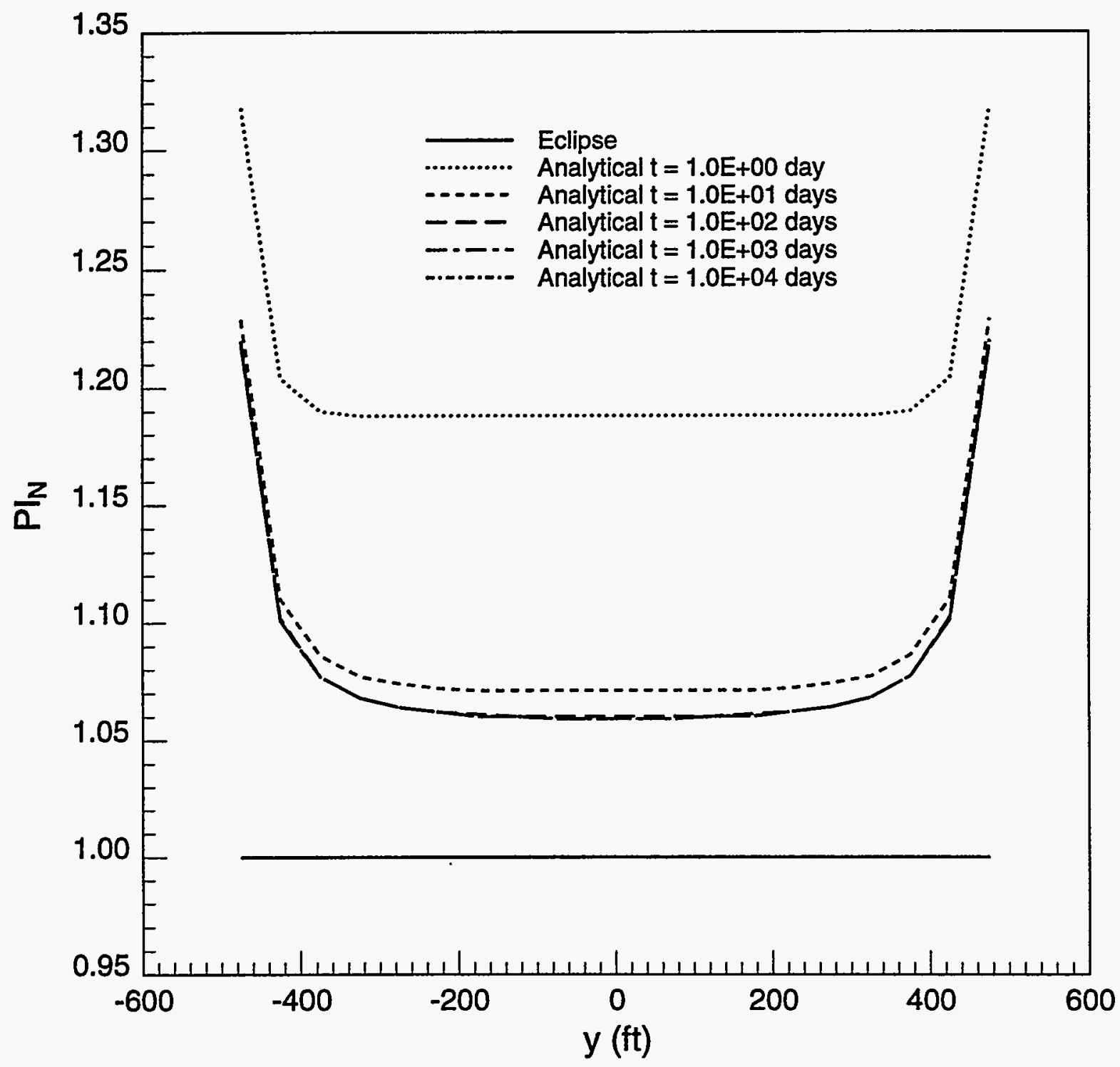

Figure 4.6: Comparison of Normalized Productivity Index 


\section{Flexible Voronoi Grids for Reservoir Simulation (Task 1)}

This chapter is written by Santosh Verma, a $\mathrm{Ph} . \mathrm{D}$. candidate under Prof. Khalid Aziz, who is developing the flexible Voronoi grid simulator.

\section{Objective}

The objective of this project is to develop a three-dimensional Voronoi grid reservoir simulator which honors horizontal/deviated wells, local flow geometry, faults, major heterogeneities, anisotropy, etc. The mathematical formulation for the flow simulation should take into account such a complex gridding scheme. Pressure and flow calculations in a wellbore (obtained from a wellbore simulator) will be coupled with similar reservoir variables.

\subsection{Introduction}

One of earliest methods used in reservoir simulation to obtain flexibility in gridding was local Cartesian grid refinement (LCGR). Figure 5.1 shows the use of fine grids inside a coarse grid. LCGR can thus be used in regions which require finer resolution. A sharp saturation front, a region of widely varying petrophysical properties, flow geometry near a wellbore, etc., are cases where LCGR can be used. Some disadvantages of this method are

- Line connecting grid points in the coarse region to those in the refined are not always orthogonal to the boundary between them.

- Potential gradients at the coarse-fine block boundaries become dependent on potentials of a few more blocks than is the case for regular grid, thus increasing the bandwidth of the Jacobian matrix.

- Static LGR is also not the best option for simulation problems which have saturation fronts moving between injectors and producers.

To obtain radial flow geometry near a well, hybrid local grid refinement [32] is quite popular. Figure 5.2 shows such a grid. These grids provide an accurate flow description very near the well. They also result in a very simple well model. Orthogonal curvilinear grids are used in the well region and Cartesian grids are used in the reservoir region. Some disadvantages of this method are

- Irregularly shaped blocks (pseudoblocks) are used at the boundary of the well and reservoir regions. These pseudoblocks give rise to the use of two different grid points to compute flow between the pseudoblock and curvilinear grid and that between pseudoblock and Cartesian grid. 


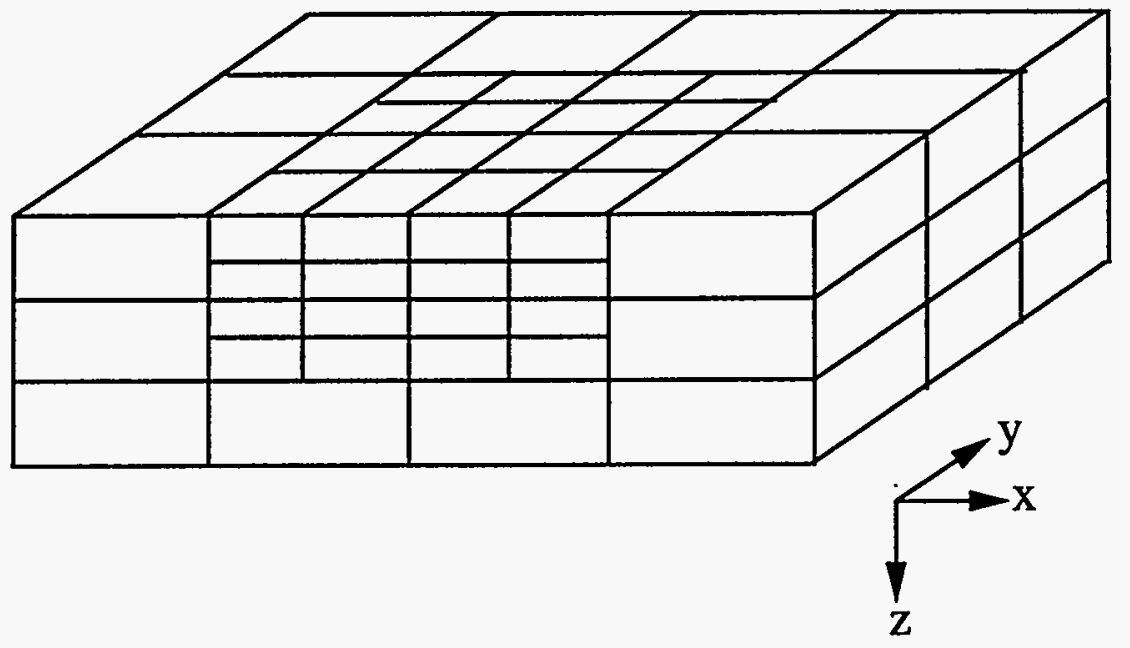

Figure 5.1: Cartesian Local Grid Refinement

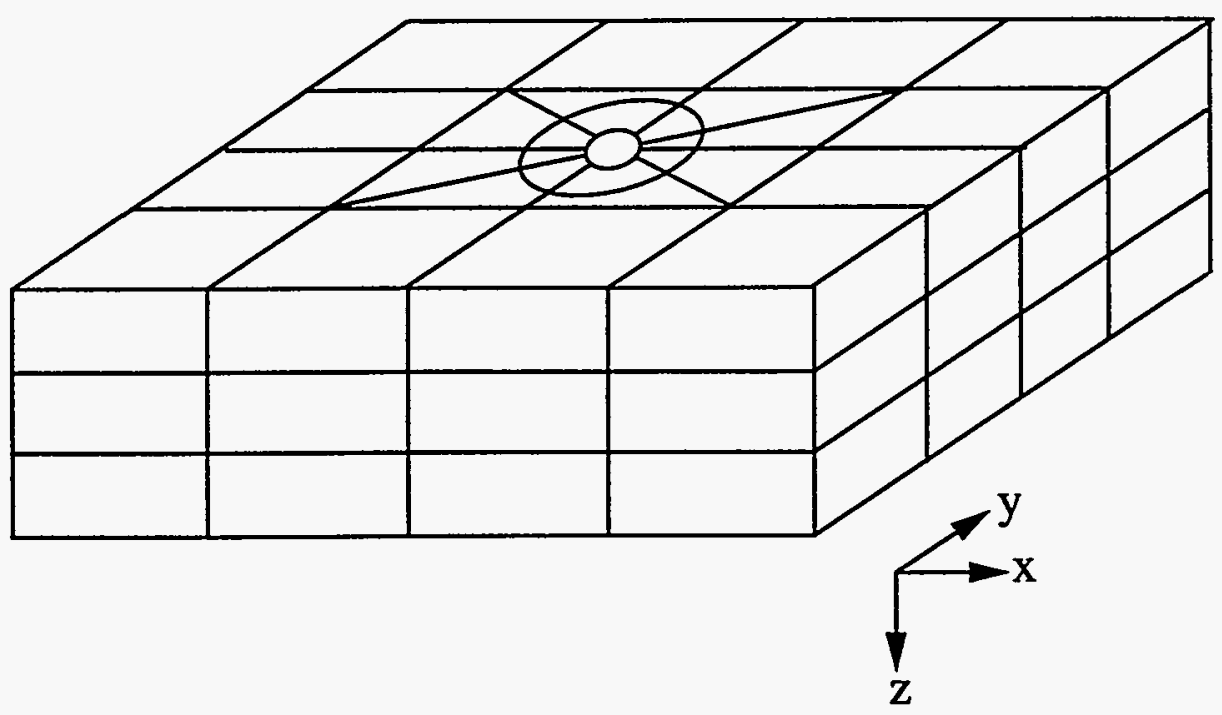

Figure 5.2: Hybrid Local Grid Refinement 


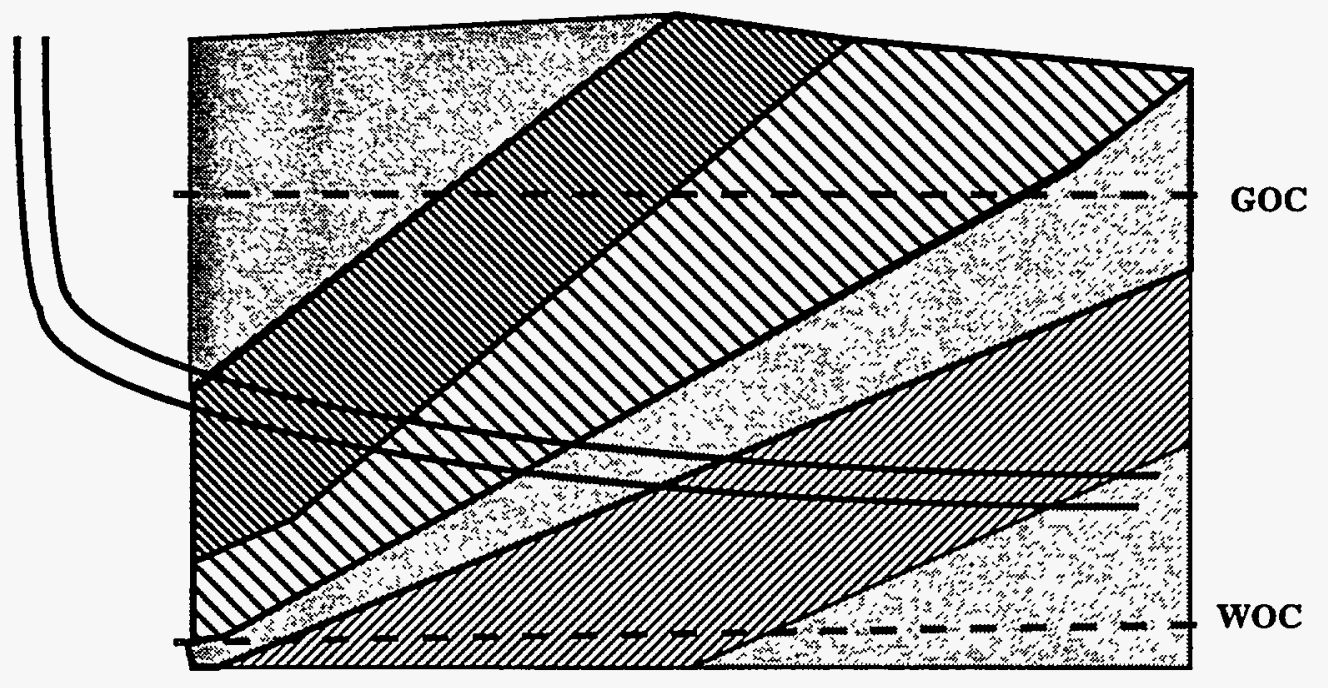

Figure 5.3: A Horizontal Well Through a Few Tilted Layers

- Some approximation is needed to handle transmissibility at the interface.

Both the Cartesian and hybrid LGR require a base grid which is Cartesian. Hence it becomes difficult to align the grid to conform to varying facies, horizontal wellbores, etc., even in the horizontal plane. Use of Voronoi grids has been proposed to tackle this problem by Palagi [18].

Use of Voronoi grids facilitates the specification of grid points anywhere inside the domain. Grids are locally orthogonal, hence enabling a reasonably accurate computation of interblock transmissibility for heterogeneous but isotropic permeabilities, providing there is no sub-grid variability within each Voronoi grid block. A practical and easy way was proposed to generate the grids in the horizontal (bedding) plane by Palagi. The location of grid points is determined using modules. Voronoi blocks which conform to this distribution of grid points are then constructed.

The Voronoi grid methods implemented previously have several limitations. These methods have been developed for heterogeneous but isotropic reservoirs and used only for the horizontal (bedding) plane. Variations in properties in the vertical (dip normal) direction are handled by dividing the reservoir into layers with the same horizontal grids used in all the layers. The simulation scheme using Voronoi grids, as it stands today, lacks 3-D flexibility in modeling horizontal/inclined wells which may pass through layers of widely varying properties. Heterogeneities, faults, local flow geometry, etc., which are not normally of two-dimensional nature can not be modeled with the existing scheme. The complex geology in Figure 5.3 (only a cross-section is shown) is an example where the existing capability is unsuccessful.

A new code is being developed in $\mathrm{C}++$ language for a 3D 3-phase black oil simulator with more general Voronoi capabilities. In the first version of this, 2D Vornoi grids will be allowed in the horizontal plane in some parts of the reservoir, with other regions having vertical 2D-Voronoi gridding, as shown in Figure 5.4. The fully generalized 3D-Voronoi gridding will be exploited later. 


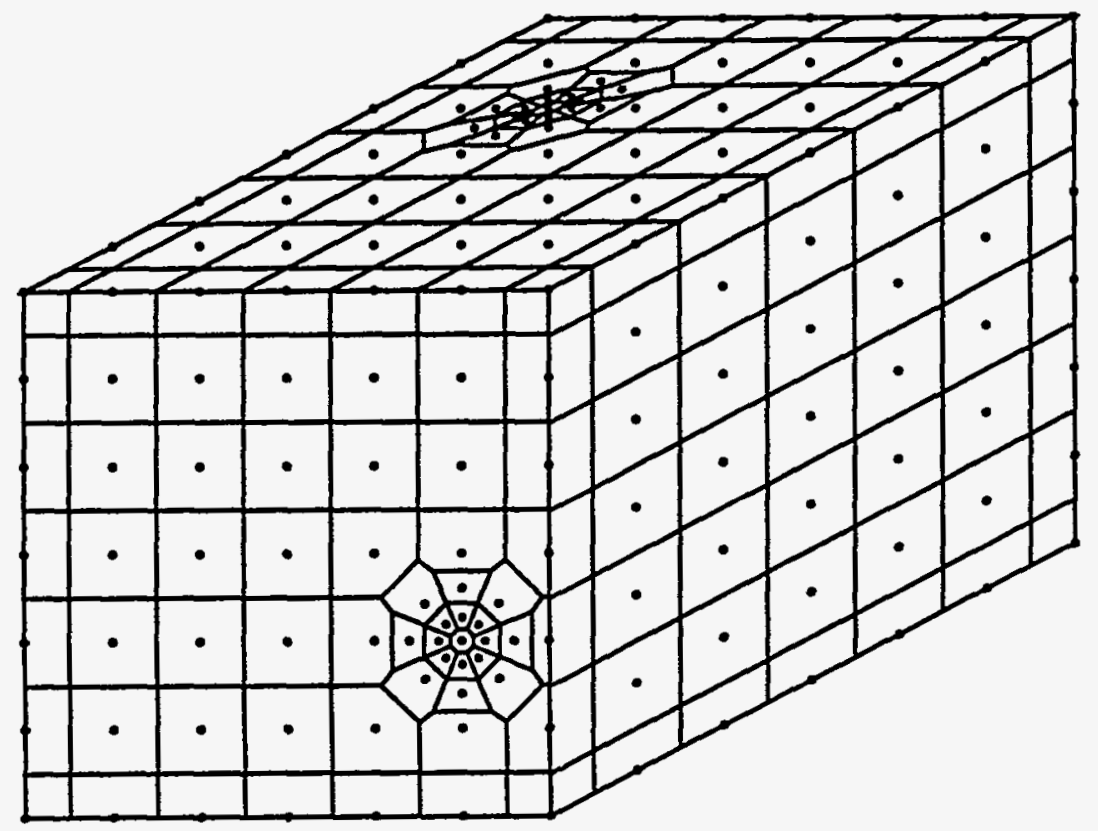

Figure 5.4: Example of Use of 2-D Voronoi Grids Representing Horizontal and Vertical Wells

\subsection{Mathematical Model}

The two most common mathematical models used with flexible grids are Control Volume Finite Difference (CVFD) or Control Volume Finite Element (CVFE). Both schemes are derived from the same integral formulation. CVFD is used with orthogonal grids. Since Voronoi grids are locally orthogonal CVFD formulation is used in this work. The CVFE gives more flexibility in grid generation, but its use in simulation of multiphase flow causes a problem because determination of upstream weighted fluxes becomes difficult. The following description of the CVFD scheme has been taken from Palagi.

Eq. 5.1 represents the application of the conservation law to an arbitrary component $\vec{c}$ in a control volume $V$ with an external area $A$ :

$$
-\oint_{A} \sum_{p=1}^{N_{p}} x_{\bar{c} p} \rho_{p} \vec{v}_{p} \cdot \vec{n} d A=\frac{\partial}{\partial t} \int_{V} \sum_{p=1}^{N_{p}} x_{\bar{c} p} \rho_{p} S_{p} \phi d V
$$

For a stationary control volume, i.e., a volume that is invariant in time, the integral on the right hand side can be expressed as:

$$
\frac{\partial}{\partial t} \int_{V} \sum_{p=1}^{N_{p}} x_{\bar{c} p} \rho_{p} S_{p} \phi d V=\int_{V} \sum_{p=1}^{N_{p}} \frac{\partial}{\partial t}\left(x_{\bar{c} p} \rho_{p} S_{p} \phi\right) d V
$$

The application of the divergence theorem (Bird et al.[33], 1960 pages 731-2) to the left hand side of Eq. 5.1 results in the following: 


$$
\oint_{A} \sum_{p=1}^{N_{p}} x_{\bar{c} p} \rho_{p} \vec{v}_{p} \cdot \vec{n} d A=\int_{V} \sum_{p=1}^{N_{p}} \nabla \cdot\left(x_{\bar{c} p} \rho_{p} \vec{v}_{p}\right) d V
$$

Substituting Eq. 5.2 and Eq. 5.3 in Eq. 5.1 yields:

$$
\int_{V}-\sum_{p=1}^{N_{p}} \nabla \cdot\left(x_{\bar{c} p} \rho_{p} \vec{v}_{p}\right) d V=\int_{V} \sum_{p=1}^{N_{p}} \frac{\partial}{\partial t}\left(x_{\bar{c} p} \rho_{p} S_{p} \phi\right) d V
$$

The control volume $V$ is arbitrary; therefore, the integrands on both sides of Eq. 5.4 have to be equal, and the final form of the differential equation is:

$$
-\sum_{p=1}^{N_{p}} \nabla \cdot\left(x_{\bar{c} p} \rho_{p} \vec{v}_{p}\right)=\sum_{p=1}^{N_{p}} \frac{\partial}{\partial t}\left(x_{\bar{c} p} \rho_{p} S_{p} \phi\right)
$$

The discretized form of the flow equation (Eq. 5.5) for each component $\bar{c}$ is

$$
\begin{gathered}
\sum_{j=1}^{N_{n}} \sum_{p=1}^{N_{p}} T_{i j}\left(\overline{x_{\bar{c} p} \rho_{p}}\right)_{i j}^{n+1}\left(\frac{k_{r p}}{\mu_{p}}\right)_{i j}^{n+1}\left(\Phi_{p, j}-\Phi_{p, i}\right)^{n+1}= \\
\frac{V_{b, i}}{\Delta t} \sum_{p=1}^{N_{p}}\left[\left(x_{\bar{c} p} \rho_{p} S_{p} \phi\right)^{n+1}-\left(x_{\bar{c} p} \rho_{p} S_{p} \phi\right)^{n}\right]_{i}+\sum_{k=1}^{N_{w}} \sum_{p=1}^{N_{p}}\left(x_{\bar{c} p} \rho_{p} q_{p}\right)_{i, k}^{n+1}
\end{gathered}
$$

Eq. 5.6 is the final form of the discretized equation for a multiphase multicomponent system using the CVFD method. Note that the only parameters that depend on the grid geometry are $T$ and $V_{b}$, which are independent of the fluid properties. The transmissibility factor $T$ is defined by

$$
T_{i j}=\left(\frac{A k}{D}\right)_{i j}
$$

where $A$ is the area open to flow, $D$ is the distance and $k$ is the permeability between the $i$ and $j$ blocks. Determining $k_{i j}$ for the connection is one of the major challenges for three-dimensional Voronoi grid scheme for an anisotropic system.

\subsection{Handling Anisotropy}

As mentioned earlier determining $k_{i j}$ to be used in computing the interblock transmissibility, for a three-dimensional Voronoi gridding method, is not straightforward. A method to handle this problem is shown now. The disadvantages of this method will also be mentioned. The derivation shown here is for two-dimensional problem, but it can be easily extended for a three-Dimensional case. It is assumed that $k_{x}$ and $k_{y}$ are known at the grid point locations, and $x$ and $y$ lie along the principal permeability directions for the homogeneous but anisotropic medium.

In an anisotropic system the direction of flux and gradient are not necessarily the same, see Figure 5.5. Let $\vec{q}$ denote the flux, $\Delta \vec{\Phi}$ the gradient and $\vec{n}$ the direction of the outward normal. Let $\Phi_{n}$ denote the potential gradient along the normal and $q_{n}$ the flux along the normal. Then 


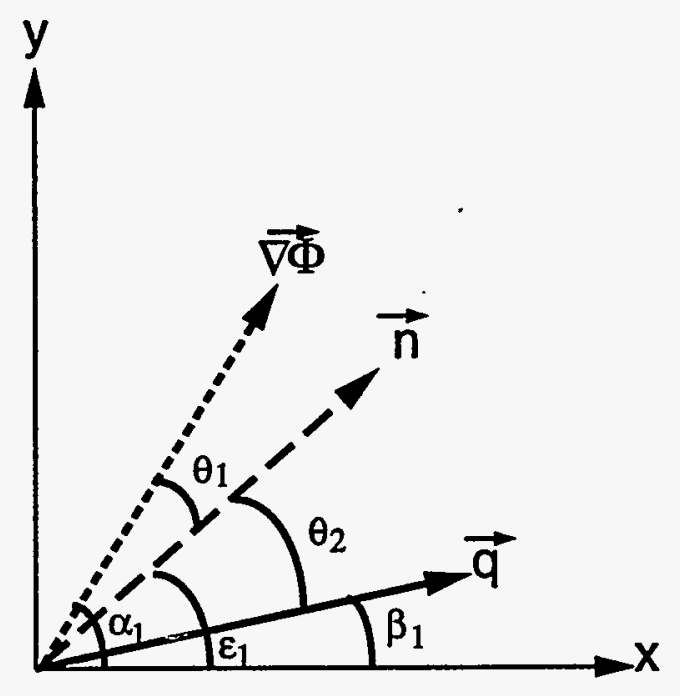

Figure 5.5: Gradient and Flux in an Anisotropic System

$$
q_{n}=\vec{q} \cdot \vec{n}=k_{n} \Phi_{n}=k_{n} \nabla \vec{\Phi} \cos \theta_{1}
$$

where $k_{n}$ is the equivalent permeability along the normal, and $\theta_{1}$ is the angle between the normal and the direction of the potential gradient. We can write $q_{n}$ also as

$$
q_{n}=k_{x} \Phi_{x} \cos \epsilon_{1}+k_{y} \Phi_{y} \cos \epsilon_{2}
$$

where $\cos \epsilon_{1}$ and $\cos \epsilon_{2}$ are the direction cosines of the normal. Using Eq. 5.8 and Eq. 5.9 we can arrive at an expression for the equivalent permeability in $\vec{n}$ direction as

$$
k_{n}=\frac{k_{x} \cos \alpha_{1} \cos \epsilon_{1}+k_{y} \cos \alpha_{2} \cos \epsilon_{2}}{\cos \left(\alpha_{1}-\epsilon_{1}\right)}
$$

In the above equation $\cos \alpha_{1}$ and $\cos \alpha_{2}$ are given by

$$
\begin{aligned}
& \cos \alpha_{1}=\frac{\Phi_{x}}{\sqrt{\Phi_{x}^{2}+\Phi_{y}^{2}}} \\
& \cos \alpha_{2}=\frac{\Phi_{y}}{\sqrt{\Phi_{x}^{2}+\Phi_{y}^{2}}}
\end{aligned}
$$

$q_{n}$ can then be easily computed from Eq. 5.8. To arrive at the value of potential derivatives, an expression for $\Phi$ has to be obtained on the surface of the Voronoi block. Once this is known the flow normal to the particular surface can be easily determined. One of the major disadvantages of this approach is in estimation of the potential derivatives at the surface. Since there may be too many grid nodes involved in this expression, the resulting Jacobian matrix will contain more entries than what is obtained with a standard grid. However, the efficiency of a scheme which uses explicit computation of the equivalent permeability tensor needs to be tested. 


\subsection{Generation of Voronoi Grids}

Palagi presented a Voronoi grid generation scheme on a plane. A scheme to generate the grids in three-dimension is presented in this section. This scheme has been adapted from those available in the literature. The source code written during the course of this work uses a public domain software Detri [34] to generate the three-dimensional Delaunay triangulation. Description of the source code developed by the authors of this study is given in the Appendix.

For a set $S=\left\{p_{1}, p_{2}, \ldots, p_{n}\right\}$ of $n$ points in Euclidean 3 -space, $R^{3}$, the associated Voronoi diagram is a sequence $V\left(p_{1}\right), V\left(p_{2}\right), \ldots, V\left(p_{n}\right)$ of convex polyhedra covering 3-space, where $V\left(p_{i}\right)$ consists of all points of 3-space having $p_{i}$ as a nearest point in the set $S$ (see Avis and Bhattarcharya [35]). Thus

$$
V\left(p_{i}\right)=\left\{x \in R^{d}: d\left(x, p_{i}\right) \leq d\left(x, p_{j}\right), \quad j=1,2, \ldots, n\right\}
$$

where $d\left(x, p_{i}\right)$ denotes the Euclidean distance between the points $x$ and $p_{i}$. The locus of all points at least as close to $p_{i}$ as to $p_{j}$ is the closed half-space $H\left(p_{i}, p_{j}\right)$, associated with $p_{i}$, bounded by the perpendicular bisector hyperplane $B\left(p_{i}, p_{j}\right)$ of the line segment $p_{i}$ and $p_{j}$. The locus of all points having $p_{i}$ as the nearest point is the intersection of all half-spaces associated with $p_{i} . V\left(p_{i}\right)$ is a convex polyhedron. The Voronoi diagram has a dual that has been called the Delaunay triangulation. This structure is defined directly on the set of data points $S$. Two points $p_{i}$ and $p_{j}$ are called Delaunay neighbors if their corresponding Voronoi polyhedra intersect in a face.

As a starting point, the Delaunay triangulation of a set of points is constructed using Detri. A set of tetrahedra is obtained. The points forming the tetrahedra can also be interpreted as the neighbor connections to be used in simulation. The perpendicular bisector hyperplane of each of the edges of a tetrahedron itersect at a point. This intersection point also forms a vertex of the Voronoi polyhedron associated with each of the points of the tetrahedron. As long as each of the tetrahedra which has a point $p_{i}$ as its vertex which lies inside the overall domain, the intersection point of the perpendicular bisector hyperplane of each tetrahedron completes the set of vertices associated with the Voronoi polyhedra of point $p_{i}$. Figure 5.6 shows a tetrahedron with four grid nodes at its vertices and the associated Voronoi block for the nodes.

If the intersection point lies outside the tetrahedron, and the tetrahedron contains faces which are on the boundary of the domain, the Voronoi vertex may lie out of the domain. The algorithm used in this work takes this into account and each such Voronoi polyhedron is suitably adjusted so that no part of it lies outside the domain. The $\mathrm{C}++$ objects used in the program to obtain the Voronoi polyhedra are described in the Appendix.

One of the major challenges in this work is to align the Voronoi polyhedra along layers, faults, horizontal wells, etc. It is indeed quite difficult to devise a scheme which is as computationally inexpensive as a traditional Cartesian grid simulator. One possible approach is proposed in this study. This method has however not yet been implemented in the simulator being developed at Stanford. It is important that a proper description of the facies distribution exist before gridding is attempted. It is expected that the bounding surface of each facies to be modeled be described by a set of points on the surface. The grid points will be put on these boundaries. The distance between the points on the boundary will 


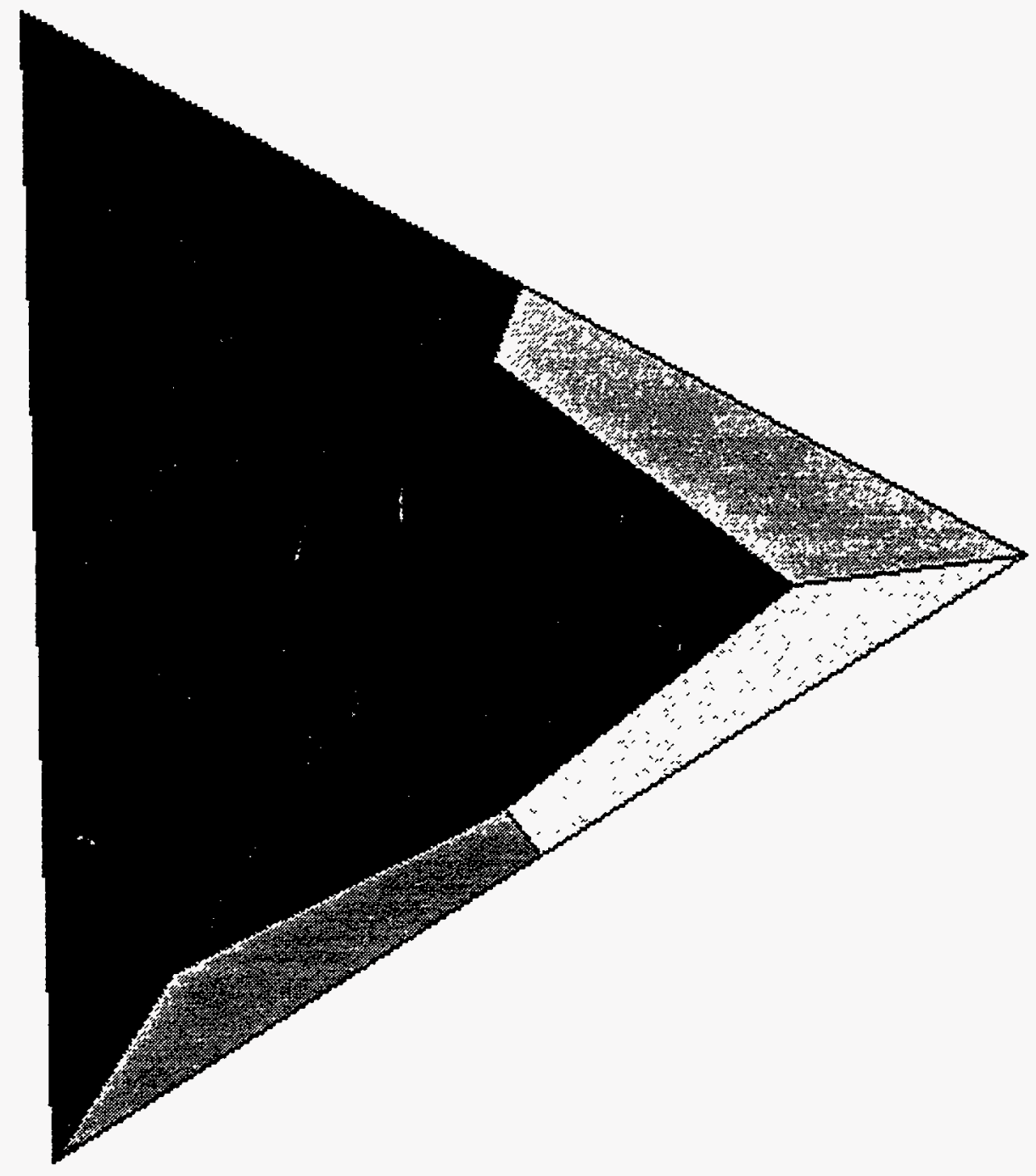

Figure 5.6: Intersecting Faces of Voronoi Blocks on Three Sides of a Basic Tetrahedron Joining Four Mesh Points in a 3D-Grid 
depend on the curvature of the surface, the error which the user can tolerate in representing the surface by a set of planes, and the minimum and maximum distance between the grid points in the horizontal and vertical directions. Points can also be located inside a bed, depending on the user's judgement. Voronoi polyhedra can then be constructed around the grid points located in such a manner. Since the potential gradients can be very steep just near the boundary of two facies of highly contrasting permeabilities, a single point at the boundary can not represent the volumetrically averaged properties like pressure, fluid saturation, of both facies associated with the point. Hence, it is proposed that one might use two property points at the same physical location. An appropriate function connecting the flow between the two points can then be developed. The disadvantage in using this method is the increase in the number of unknowns to be determined. However, this increase can easily be offset by the decrease in the number of grid points necessary to resolve complex boundaries compared to the numbered used by a Cartesian grid simulator.

The grids for a horizontal well can easily be created by using the well profile information, along with user specified parameters like the location of grid nodes around the well, option of spherical grid nodes at the well ends, etc. Faults can be handled in a way similar to the layer boundaries mentioned earlier. The grid nodes at the fault locations can be made to communicate across the fault. This can be achieved by using two grid nodes at the same physical location.

\subsection{Visualization}

A geometric viewer Geomview [36] developed at the Geometry Center is used to view the grids generated. Geomview can be called from other programs. Hence the only interactive graphics planned to be developed at this stage of the study is to have a window which would interact with Geomview to manipulate the number of Voronoi grids to be viewed at one time.

\subsection{Appendix}

The appendix describes the progress made in the development of a three-dimensional three-phase black-oil reservoir simulator which incorporates three-dimensional Voronoi grid generation. The source code is being written in $\mathrm{C}++$. A number of topics connected with flexible grid generation and computer modeling of fluid flow are to be modeled in this study. The topics include grid generation algorithm, coupling flow inside horizontal/inclined well to flow in the reservoir, grid visualization, petrophysical property allocation at grid nodes, well model, reservoir initialization and solution methods. Programs specific to reservoir initialization and solution methods have already been developed. Only those $\mathrm{C}++$ classes ( a class is the means of representing an object in $\mathrm{C}++$ ) which have been programmed and tested successfully are now described. The description here is very sketchy. A manual will be written to describe the classes and their use in the reservoir simulator.

- SMatrix class represents a sparse matrix object. The SMatrix object data members include the nonzero entries of the matrix, the number of nonzero entries and some pointer arrays which are used to find the location of the matrix entries in terms of 
row and column numbers. Some of the member functions include functions for direct solution, iterative solution, computing transpose, inverse, etc. Some overloaded operators make matrix addition, subtraction, matrix product calculation, and similar operations very easy to program. A full matrix class called FMatrix represents a full matrix object. Its member functions do similar operations on matrices as those already mentioned for SMatrix class.

- CartModule class is used to represent a Cartesian module object. The data members include the number of grid points in $\mathrm{x}$ and $\mathrm{y}$ directions, and the distance between the grid points. This class is used only for generating grid points in a plane.

- CyliModule class represents a cylindrical module object. Some of the data members are the number of blocks in theta (angular coordinate) direction, number of blocks in $r$ (radial) direction, the well radius, the distance between grid nodes in $r$ and theta directions, etc.

- HexaModule class represents a hexagonal module object. Its data members include number of blocks in $\mathrm{x}$ and $\mathrm{y}$ directions. and the distance between the grid nodes.

- GlobalPoint class represents a set of grid points, which are generated by the interaction of the CartModule, CyliModule and HexaModule objects. It has a list of Point objects as data member. Member functions to manipulate the Point objects of this object have been included. They perform operations such as addition of two GlobalPoint objects, removal of Points which lie outside the domain of interest, etc.

- VoronoiBlock class represents a Voronoi Block object. Its data members include the location of the associated node, number of vertices, number of neighbors, the list of neighbor ID's, if node is at the boundary, number of faces and the description of the faces. Apart from the constructors, some of the member functions include one to compute volume of the block, another to compute a block resulting by cutting the Voronoi Block with a plane, etc.

- Point object is a very fundamental object used in this code. The data members are the $\mathrm{x}, \mathrm{y}$ and $\mathrm{z}$ coordinates of the Point object. The overloaded operator + , adds a Vector to a Point to obtain a new Point object. The operator - subtracts two Point objects to give a Vector object. Another function removes all but one of Points having the same location.

- Line class is represented by an anchor Point and a direction Vector. A member function written finds the Point intersection of two Line objects, while another tells if a Point object is on a given Line.

- Vector class is represented by a double array of values and the dimension of of the Vector. Member functions find length, direction cosines,etc. A number of overloaded operators enable vector operations to be done with ease.

- Plane class has a Point and a normal Vector as its data members. Intersection member function returns a Point object when three Planes intersect and another such function returns a Line object when two Planes intersect. 
- Face class contains a Point set as its data members. One of its member functions finds the area of a Face object in 3-D.

- Edge class has two Point ID's as its data members.

- Facet class has a list of Edge object pointers as data members.

- Tetrahedron class has a list of Facets as its data members. The Point, Edge, Facet and Tetrahedron objects are used to create VoronoiBlock objects.

- String class has a character array as its data member. It enables manipulations of strings very easy and convenient. 


\section{Production-Based Effective Vertical Permeability for a Horizontal Well in the Presence of a Stochastic Distribution of Shales (Task 2)}

This study has been conducted as a Masters Thesis by the Ph.D. student Tomomi Yamada under supervision of Prof. Thomas A. Hewett and in conjunction with Prof. F. John Fayers.

\section{Summary}

The presence of a stochastic distribution of impermeable shale sheets strongly affects the production performance of a horizontal well. Oil recovery is drastically reduced and its uncertainty is dramatically increased for a reservoir with high shale density and long correlation range. The construction of a probability distribution function (PDF) of the oil recovery on the basis of many stochastic images requires a lot of work and computation time. However, that PDF can be easily approximated if a single ranking parameter is assigned to each realization. Conventional effective vertical permeability, which is based on vertical single phase flow (Kv_eff) was tested first since it governs horizontal well productivity. This attempt turned out to be unsuccessful because of the inappropriate representation of the inner and outer boundary conditions applied to the domain. Production-based effective vertical permeability (Kp_eff), which honors both outer and inner boundary conditions, was tried next. It successfully ranked each realization in terms of oil recovery after ten years with excellent accuracy. The correlation between Kp_eff and recovery is independent of the shale density or correlation range. This study shows that it is extremely important to honor the appropriate boundary conditions to obtain a representative permeability. The conventional permeability renormalization technique, in which either no flow or constant pressure boundaries are applied to coarse grids, fails for this reason.

\subsection{Introduction}

Stochastic simulation, in combination with flow simulation, is a powerful instrument to forecast the variability of possible production behavior for a heterogeneous reservoir. On the basis of a number of equally probable stochastic realizations, one can construct a probability distribution of oil recovery by running a flow simulator on many of these images. This requires enormous amounts of work and computation time. If there were a single parameter for each realization that indicated the ranking (quantile) of oil recovery, it would be an extremely useful alternative to running flow simulations on each realization. From the distribution of this parameter one could identify a few realizations that produce desired quantiles of oil recovery. The full distribution of oil recoveries could then be approximated by running flow simulations on a selected subset of realizations spanning the range of oil recoveries. In this report, production-based absolute vertical permeability is introduced as such a parameter. An application is demonstrated for a water flooding displacement 
problem where horizontal wells are used as injector and producer. The reservoir is highly heterogeneous with a stochastic distribution of impermeable shale sheets. The study is based on eighty stochastic images produced using sequential indicator simulation for the discontinuous shale distributions.

It is well known that the productivity of a horizontal well is strongly affected by the vertical permeability of the reservoir. In particular, the presence of discontinuous shales can cause severe problems. If a large portion of a horizontal completion interval happens to be confined between impermeable barriers, the well productivity will be significantly reduced. Thus, the recovery factor of the reservoir is strongly affected by the distribution of these shale sheets. Lien and Haldorsen [37] illustrated the impact of impermeable shale barriers on the behavior of a horizontal producer. They performed case studies on a variety of discontinuous shale densities using both explicit and implicit representations. In the explicit case, a shale is represented by assigning a transmissibility multiplier to the one of the sides of a grid block. The implicit representation is done by defining an anisotropic sand permeability for the entire reservoir. They concluded that explicit representation is preferable in two phase flow problems. Methods to compute effective vertical permeability for sand/shale sequences have been proposed by several authors. Begg and King [38] proposed a simple analytical method to estimate the distribution of vertical permeability using streamtube theory. In their work, a shale is represented as an impermeable barrier with finite thickness. Desbarats [39] proposed power averaging and percolation models to estimate effective vertical permeability. He represented shales by grid blocks with a finite thickness and permeability. Although a shale is represented in various ways in these discussions, it is common to solve only the vertical linear flow problem to obtain the effective vertical permeability. Generally, the flow to a horizontal well is by no means vertical and linear. It will be horizontal at a region far from the producer and may be radial in the vicinity of the well. An appropriate effective vertical permeability must be obtained in such a way that the influence of all of the tortuous flow paths are captured correctly. This is the basis for introducing a production-based effective vertical permeability. In this paper, the formulation and utility of this value is discussed.

\subsection{Model Description}

A single phase oil reservoir of $5,000 \mathrm{ft}$ by $5,000 \mathrm{ft}$ with a thickness of $110 \mathrm{ft}$ is discretized into $20 \times 20 \times 12$ grid blocks. The bedding is parallel to the horizontal plane. Two injectors are placed at the bottom of two opposite sides and one producer is placed at the top center. All of the wells are horizontal and parallel, with $2,000 \mathrm{ft}$ of horizontal completion interval. Water is injected from the horizontal injectors and oil is swept from the sides to the center top of the reservoir. This configuration takes some advantage of gravity, which may improve volumetric displacement. The wells are operated under fixed bottomhole pressure. The injection pressure is $5,000 \mathrm{psi}$ and the flowing pressure of the producer is $2,500 \mathrm{psi}$. No limits are put on the production or injection rate, since they may mask the correlation between effective vertical permeability and oil recovery. The model geometry is shown in Fig. 6.1 Relative permeability is based on a field core sample. Connate water and residual oil saturation are both 0.2 . The end point values of relative permeability for water and oil are 0.400 and 0.701 , respectively. Other fluid and rock properties are listed in Table 6.1. 
Table 6.1: Model Description for Shale Studies

\begin{tabular}{||ll||}
\hline Reservoir Area & $5000 \mathrm{ft}$ by $5000 \mathrm{ft}$ \\
Thickness & $110 \mathrm{ft}$ \\
Reservoir Depth & $8060 \mathrm{ft}$ (Center of Oil Column) \\
Gridding & $20(\mathrm{x}) \mathrm{by} \mathrm{20}(\mathrm{y}) \mathrm{by} 12(\mathrm{z})$ \\
Initial Reservoir Pressure & $3472 \mathrm{psi} @ 8000 \mathrm{ft}$ \\
Formation Porosity & 0.2 \\
Sand Permeability & $50 \mathrm{mD}$ \\
Capillary Pressure & $0 \mathrm{psia}$ \\
Formation Volume Factor of Oil & $1.2215 @ 3472 \mathrm{psia}$ \\
& $1.1945 @ 7000 \mathrm{psia}$ \\
Viscosity of Oil & $1.136 \mathrm{cP}$ \\
Depth of Producer & $8002.5 \mathrm{ft}$ \\
Depth of Injectors & $8107.5 \mathrm{ft}$ \\
Completion Length & $2000 \mathrm{ft}$ in x direction \\
Flowing Bottomhole Pressure & $2500 \mathrm{psia}$ \\
Injection Bottomhole Pressure & $5000 \mathrm{psia}$ \\
\hline
\end{tabular}

\subsection{Method to Generate Shale Distributions}

Stochastic shales are represented by assigning a zero transmissibility multiplier to the bottom side of grids containing shales. This is equivalent to giving a zero thickness to impermeable shale sheets. The shale distribution is generated with sequential indicator simulation, in which the distributions are characterized with volume fraction and variograms of grids containing impermeable shale sheets. The variogram defines a spatial correlation between such grids. Short $(250 \mathrm{ft})$ and long $(1,000 \mathrm{ft})$ horizontal ranges of correlation and five shale densities, ranging from 0.1 to 0.5 shales $/ 10 \mathrm{ft}$ are considered. The shale distribution is assumed to be independent from plane to plane. Therefore, there is no correlation in the vertical direction. Some of the realizations are shown in Fig. 6.2. The density of shale is expressed as a probability of appearance of shale per unit area. So 0.5 shales per $10 \mathrm{ft}$ means there is a 50 percent chance to hit a shale if a vertical well log is traced through a $10 \mathrm{ft}$. layer. The indicator variogram is assumed to be identical for both shale and sand. A spherical model with 0.3 of relative nugget is considered for the two typical correlation ranges mentioned above.

\subsection{Variability of Production Performance}

A total of ten cases, in terms of correlation ranges and shale densities, were prepared for the study. Eight realizations were produced for each of these cases. There are a total of eighty stochastic images of the shale distributions. Two phase flow (water displacing oil) simulations were run on all of these images. The variety of resulting production performance is illustrated in Fig. 6.3. In general, there is a plateau production period until the time of 


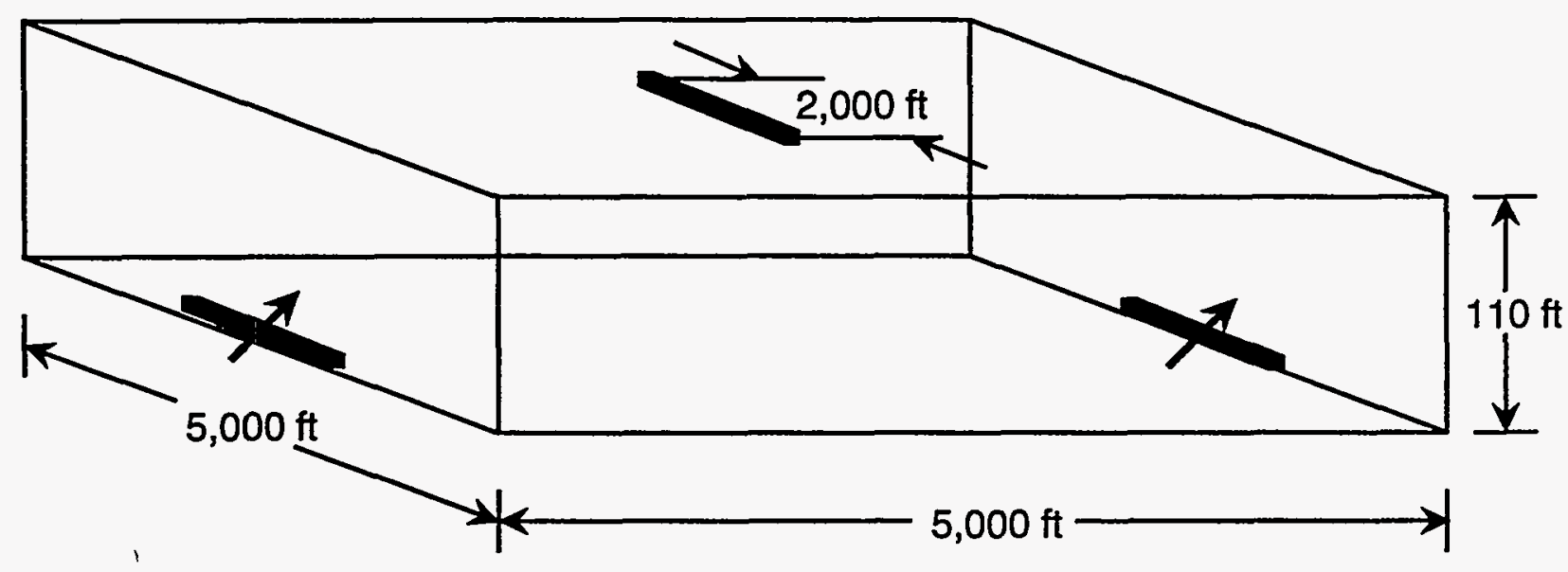

Figure 6.1: Reservoir Model

water breakthrough. During this period, the production is sustained by pressure support from the injectors. Once water breakthrough occurs, the oil rate rapidly declines due to changes in the relative permeability of oil in the vicinity of the producer. Since the producer and injectors are operated under fixed bottomhole production and injection pressures, the production rate is infinite at the very beginning of production due to pressure transients. Then it rapidly drops much below the plateau production rate until pressure support from the injectors reaches the producer. However, these periods are very short and out of the scope of this work.

Figure 6.3 shows a plot of oil rate and water cut versus time for two extreme cases, 0.1 shales $/ 10 \mathrm{ft}$ shale density with $250 \mathrm{ft}$ of correlation range, and 0.5 shales $/ 10 \mathrm{ft}$ with $1,000 \mathrm{ft}$ of correlation range. It will be easily observed that the distributed discontinuous shales significantly affect the well performance. The effect of shale density is illustrated in Fig. 6.4(a). Although low shale density does not show much effect, the well productivity is drastically reduced as shale density increases. The effect of correlation range is shown in Fig. 6.4(b). It shows that a longer correlation range amplifies the scatter of production performance. It should be understood that the larger the correlation range is, the more the system deviates from a homogeneous reservoir. It is also observed that a longer correlation range tends to reduce the well productivity at high shale density. In summary, the productivity is significantly reduced and its prediction is more difficult as shale density and correlation range become larger.

Effective Vertical Permeability Based on Linear Flow (Kv_eff) The objective of this work is to find a useful parameter to estimate the variability of the production performance. Because of its practical interest, the distribution of recovery after ten years is chosen as the ranking criterion. Effective vertical permeability was investigated first since the recovery at a certain time is related to well productivity, and well productivity is known to depend strongly on vertical permeability.

The conventional effective vertical permeability (Kv_eff) is obtained by steady state 


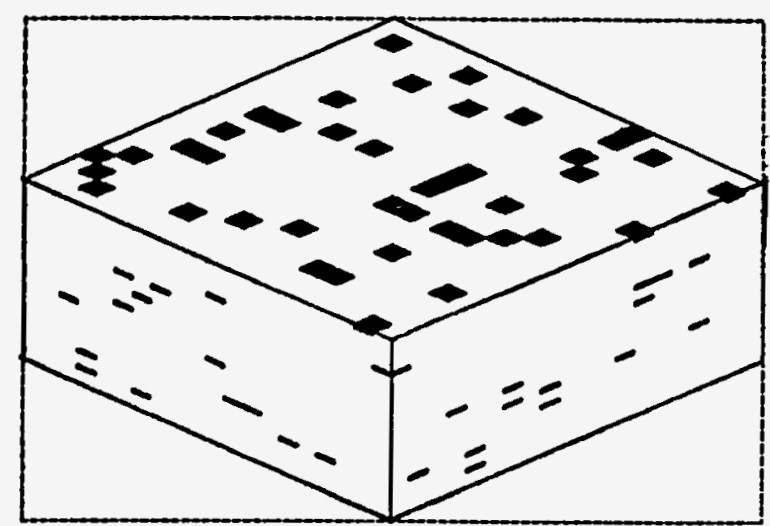

(a) 0.1 [shales/10t], Range $=250[\mathrm{t}]$

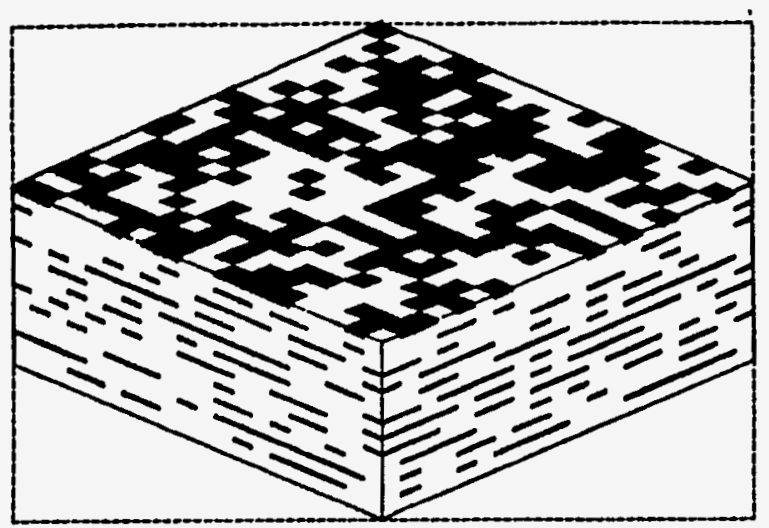

(c) 0.5 [shales/10ft], Range $=250[\mathrm{ft}]$

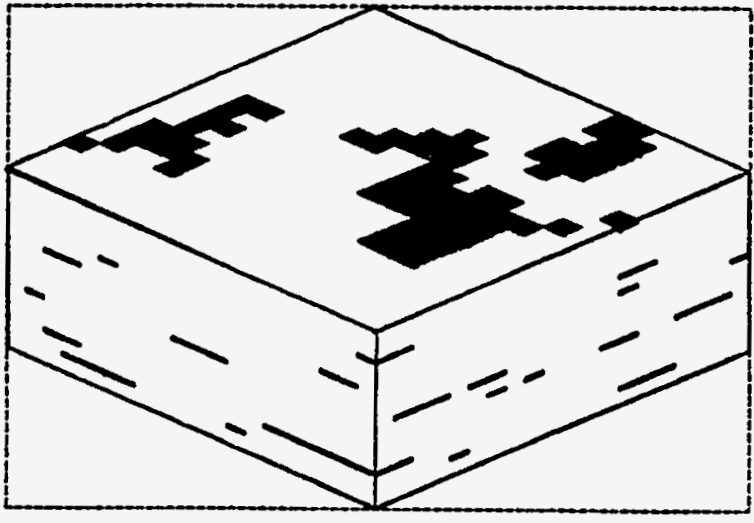

(b) 0.1 [shales/10t], Range=1000[ft]

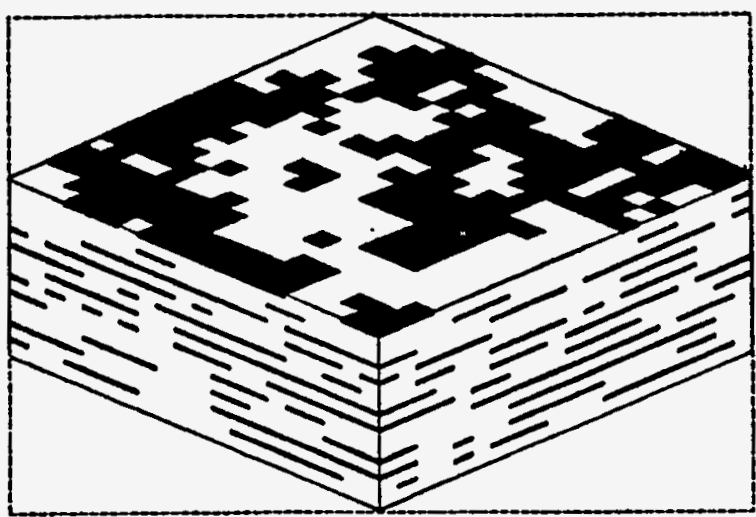

(d) 0.5 [shales/10ft], Range=1000[ft]

Figure 6.2: Stochastic Shale Distributions 


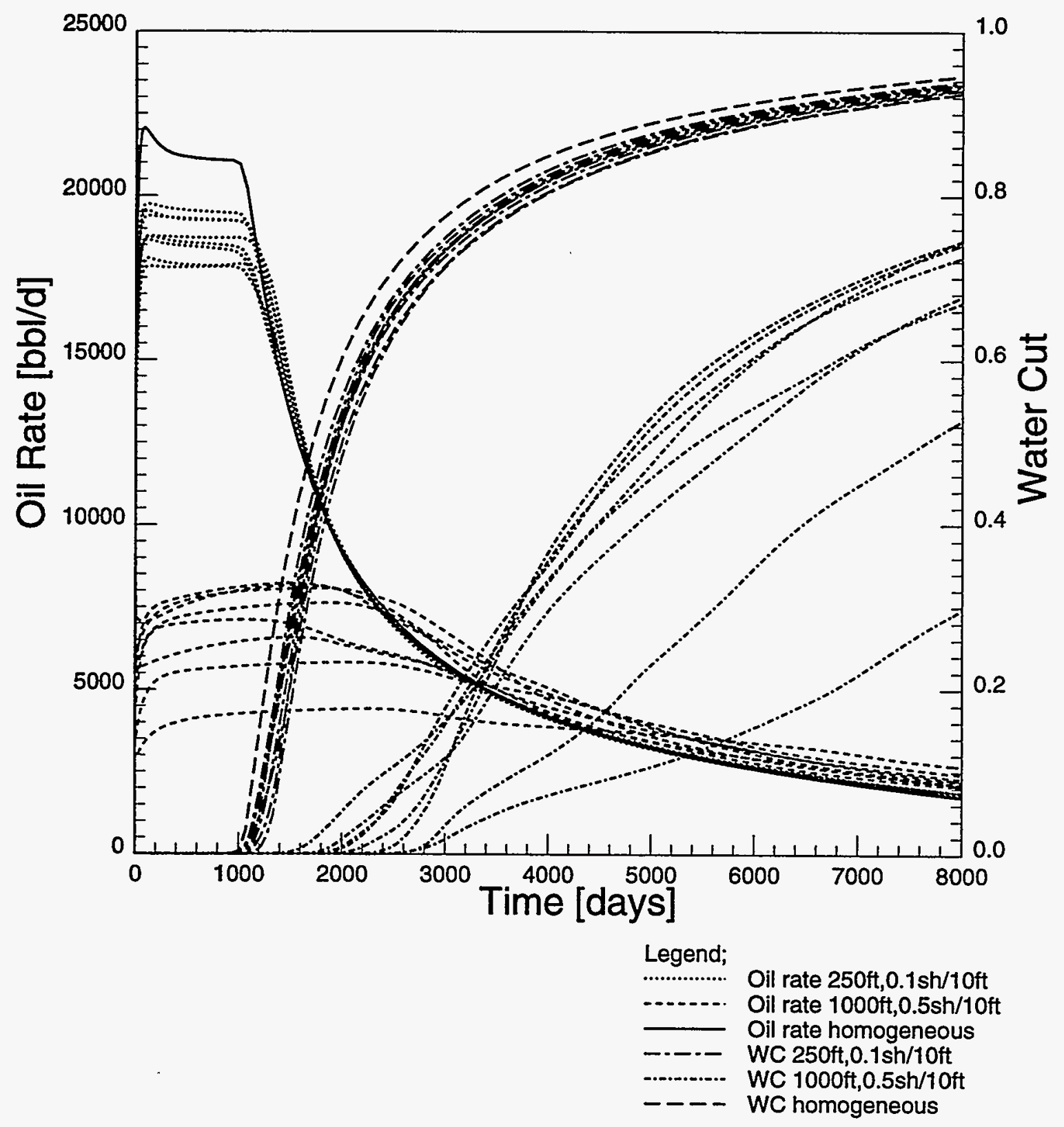

Figure 6.3: Oil Rate, Water Cut Versus Time 

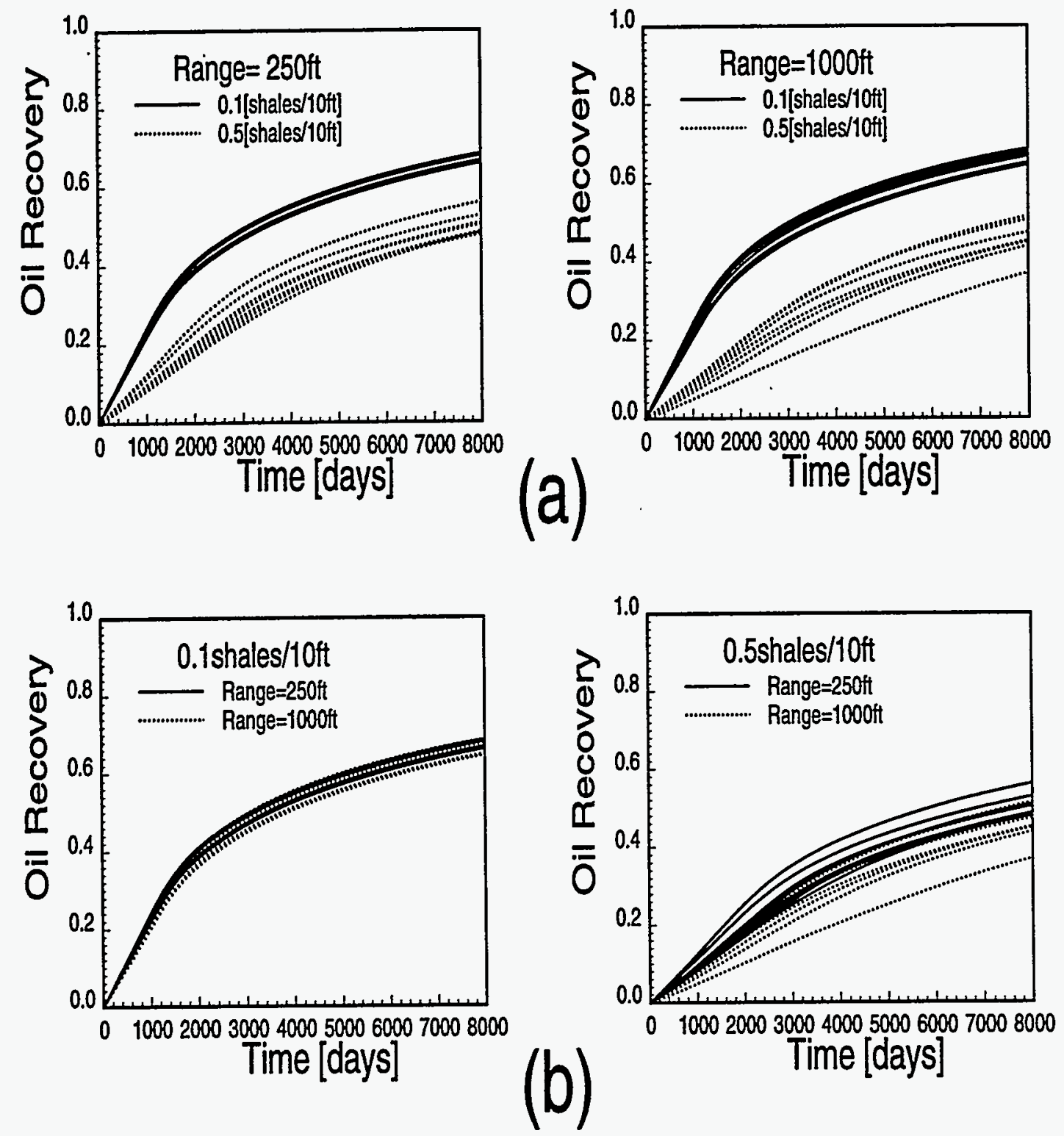

Figure 6.4: Effect of (a) Shale Density, (b) Correlation Range 
single phase flow simulation. Single phase oil is injected into the bottom plane of the reservoir and produced from the top. Kv_eff is computed from the flow potentials at the two boundaries and the total flow rate using Darcy's law. Only 100 days of flow simulation are required to obtain steady state linear flow. Figure 6.5 illustrates the relationship between shale density and the corresponding effective vertical permeability. The broken line represents the sand permeability $(50 \mathrm{md})$ times the product of the areal fraction open to flow for each plane:

$$
K_{v-\text { brokenline }}=K_{v-\text { sand }} \prod_{n=1}^{N}\left(1-f_{\text {shale }}\right)_{n}
$$

where $K_{v-b r o k e n l i n e}$ is the effective permeability represented by the broken line, $K_{v-\text { sand }}$ is the sand permeability, $f_{\text {shale }}$ is the areal fraction of impermeable shale and $N$ is the number of grid planes in the vertical direction.

If only straight flow paths are allowed to pass through the reservoir, the effective permeability will be reduced by the probability that a straight streamtube does not hit any barriers in passing through the domain. Since our model is a sequence of planes which have vertically uncorrelated shale distributions, such a situation is represented with the equation above. The existence of any non-straight flow paths will result in an increase of effective permeability. Therefore, the scatter above this line is the contribution of non-straight flow paths. It shows a wide scatter at higher density and longer correlation range which indicates that the flow is more tortuous in these realizations. Flow paths are determined by the density and the pattern of distributed shales. Figure 6.5 shows that the pattern of shales is very important. The correlation between Kv_eff and oil recovery after ten years is shown in Fig. 6.6. Although there is some correlation, the scatter of the recovery for the same Kv_eff exceeds 0.1, which for low values of recovery factor represents an unacceptably high percentage of scatter. Thus, $\mathrm{Kv}_{\text {_eff }}$ is not useful for ranking the recovery.

\subsection{Production-Based Effective Vertical Permeability (Kp_eff)}

Since the effect of shales on the productivity is not uniform over the reservoir, it is also necessary to model the non-uniformity of this impact. One solution is to use the same flooding pattern or boundary conditions, and the resulting productivity to obtain an effective permeability, Kp_eff. Since oil recovery at a certain time is strongly related to well productivity, Kp_eff is expected to have a good correlation with recovery. Here Kp_eff is defined as the equivalent homogeneous vertical permeability that results in the same productivity of a well as is produced in each stochastic shale realization. Well productivity is obtained using single phase steady state flow under fixed bottomhole pressures. A plot of oil rate against anisotropic $\mathrm{Kv}$ is used as a calibration to convert well productivity into Kp_eff (See Fig. 6.7). For example, if the stabilized single phase oil production rate is 15,000 $\mathrm{bbl} / \mathrm{d}$, it is equivalent to the case where the reservoir had $3.6 \mathrm{md}$ of anisotropic homogeneous vertical permeability, which is defined as Kp_eff. Since the existence of shales does not affect horizontal flow, the horizontal permeability is left as that of sand.

The plot of this production-based vertical permeability with recovery factor at ten years shows an excellent correlation in Fig. 6.8(a). The scatter of recovery for the same Kp_eff is within a few percent, where recovery itself is more than forty percent. This is 


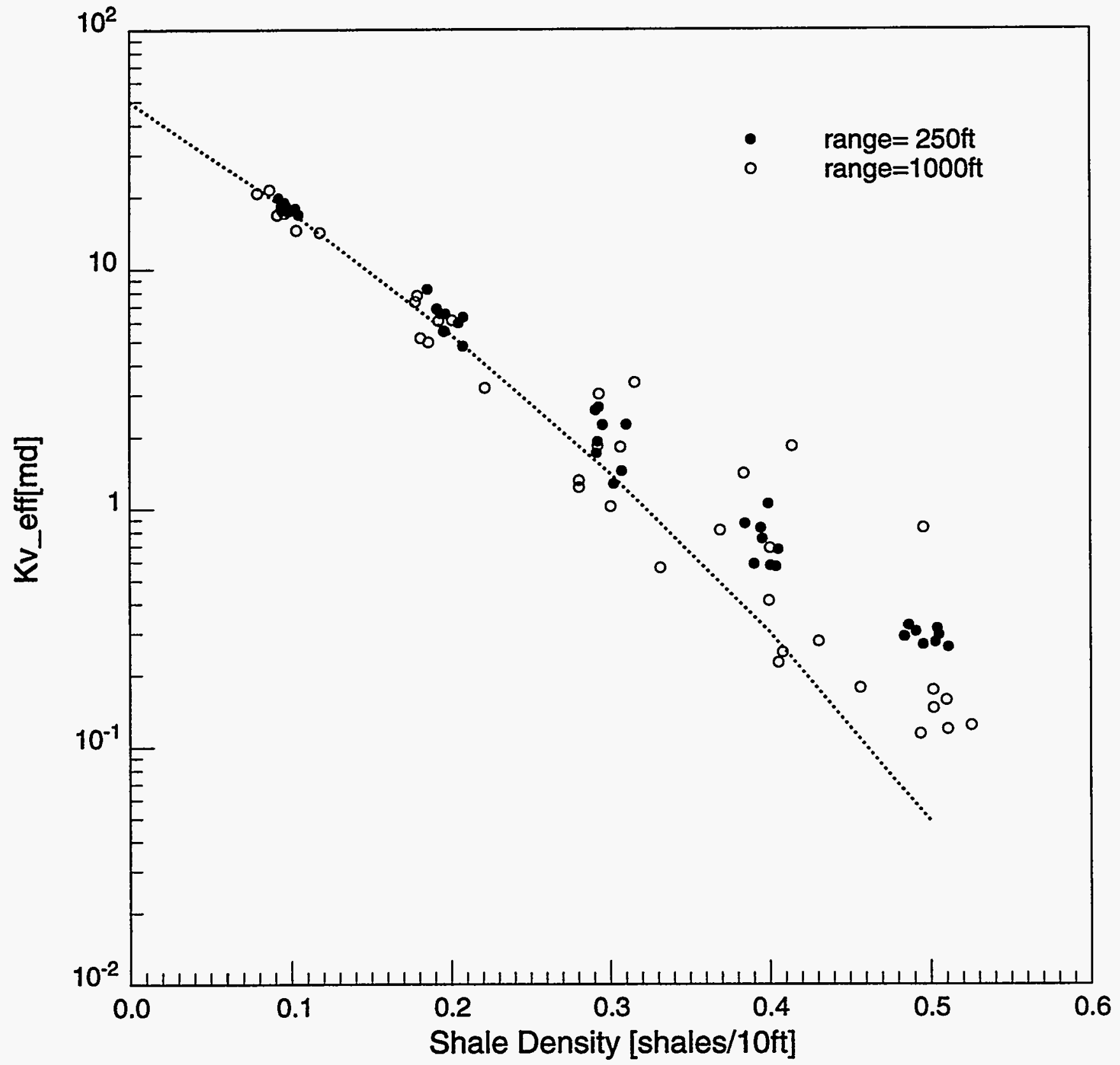

Figure 6.5: Variations of Kv_eff with Shale Density 


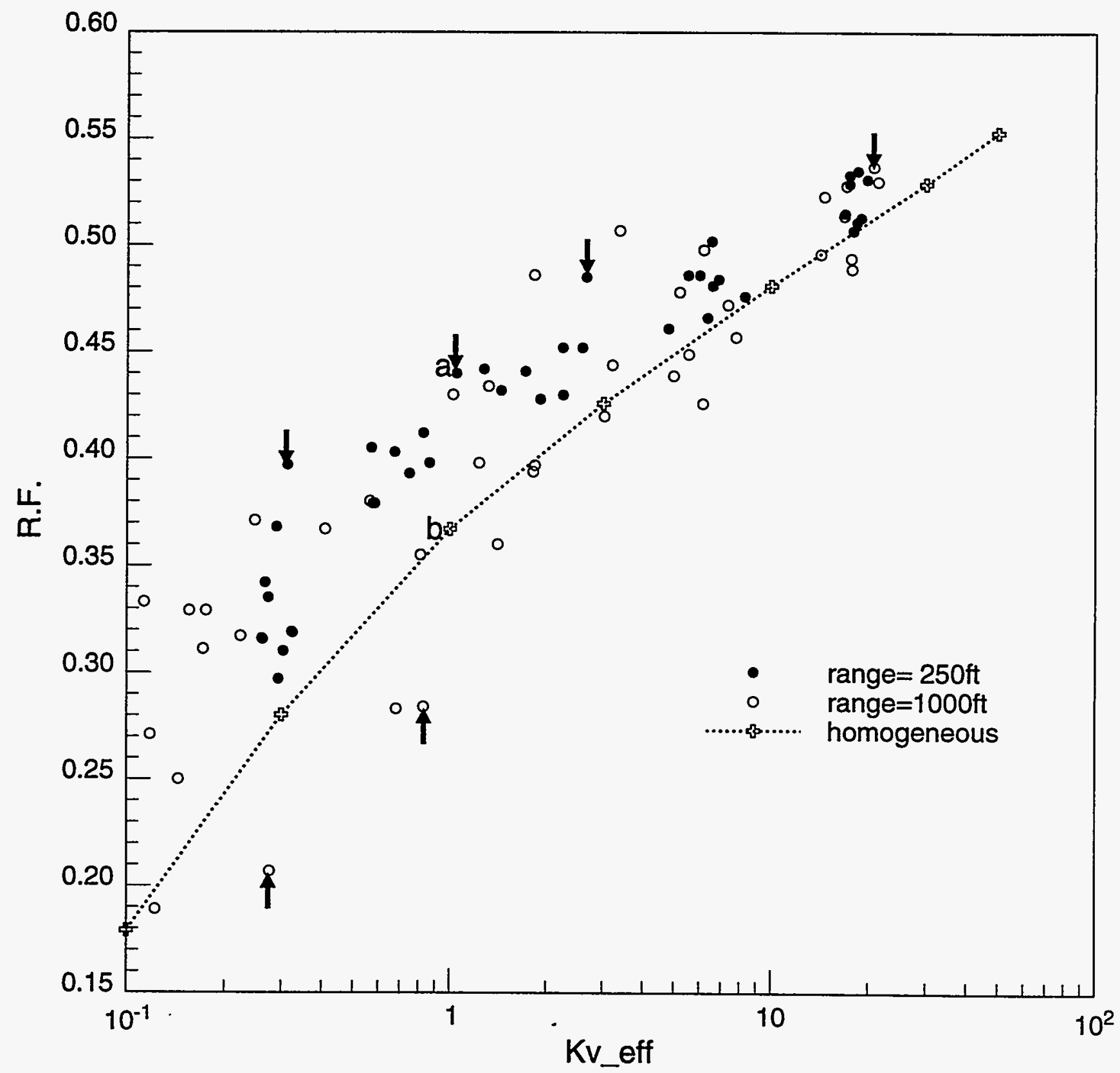

Figure 6.6: Oil Recovery Versus Kv_eff 


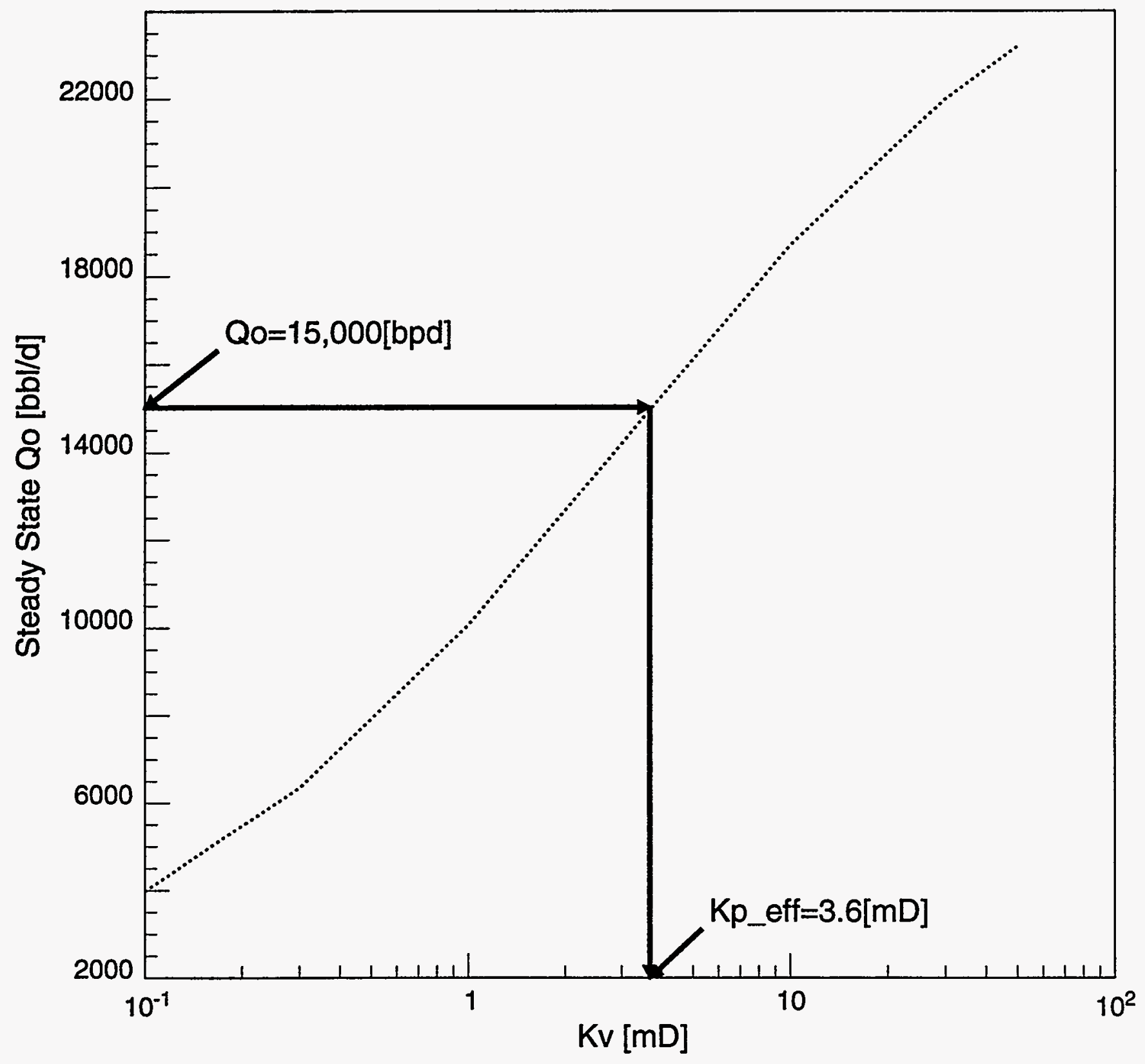

Figure 6.7: Calibration for Kp_eff 


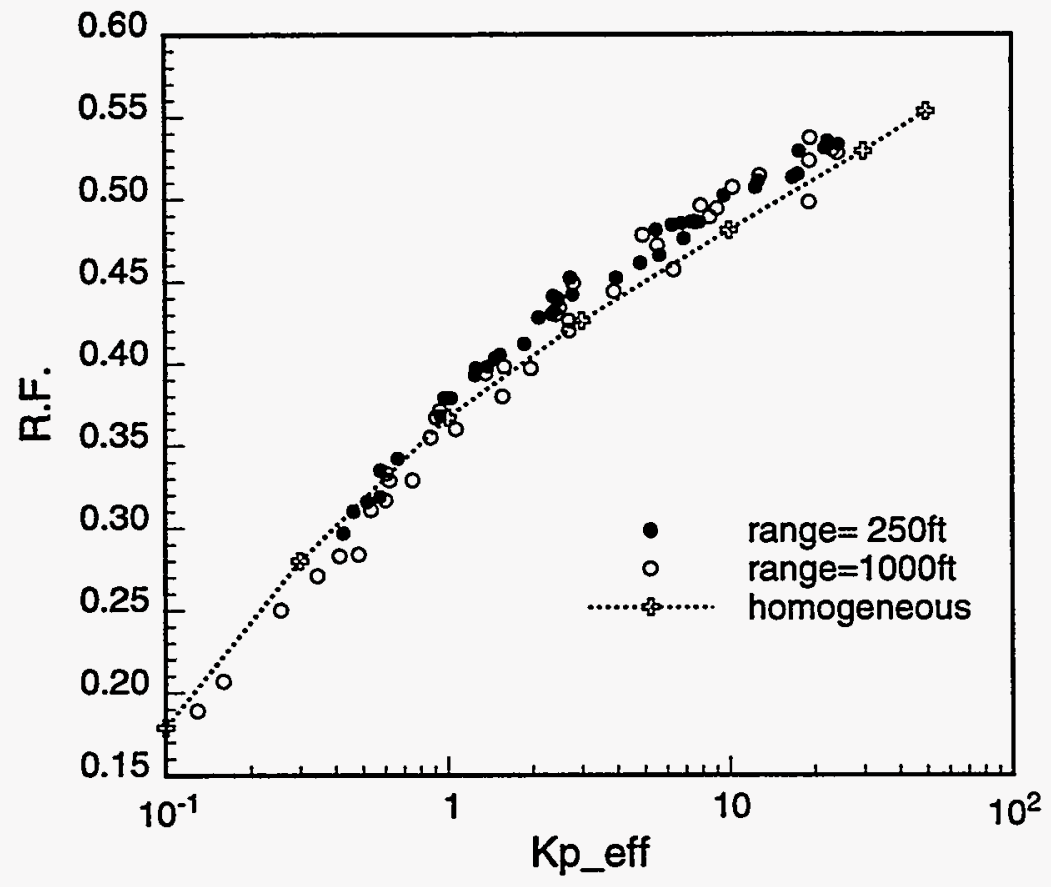

(a) R.F. vs Kp_eff (Oil Viscosity=1.136[cP])

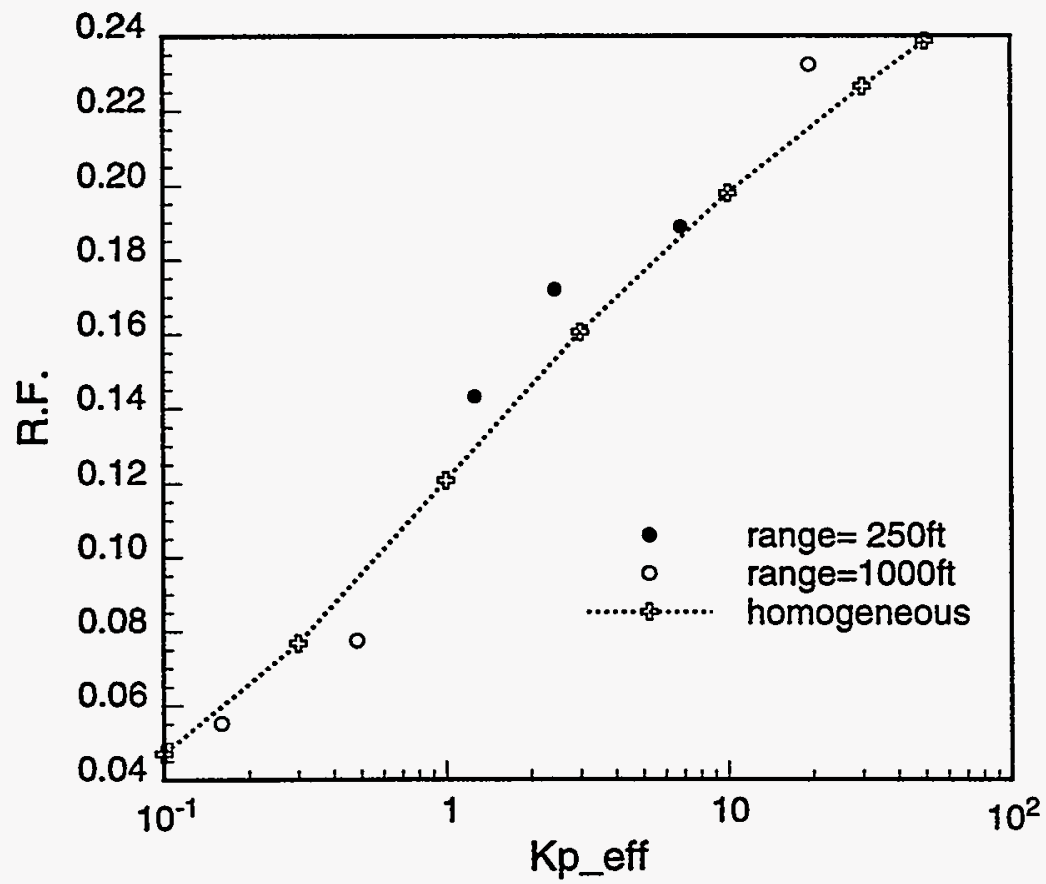

(b) R.F. vs Kp_eff (Oil Viscosity=11.36[cP])

Figure 6.8: Oil Recovery Versus Kp_eff 
sufficiently accurate for practical use. Figure 6.8(b) shows similar results for an adverse mobility ratio case where the oil is ten times as viscous as in the original case. The extreme points in Fig. 6.6 (marked with arrows) were picked for this plot. Although the oil recovery is much lower, an acceptable correlation is preserved. The most important feature of Fig. 6.8 is that oil recovery is a monotonic increasing function of Kp_eff. This characteristic makes it possible to give a ranking to each realization without running long term two phase flow simulations. Once we have determined Kp_eff, we know the quantile of oil recovery of a particular realization from its Kp_eff value. It is much easier to obtain the values of Kp_eff than to establish the recovery directly. Another important observation is that both short range $(250 \mathrm{ft})$ and long range $(1,000 \mathrm{ft})$ results are on the same curve. In other words, oil recovery is a function of Kp_eff, independent of the correlation range. Therefore, Kp_eff is a single indicator that provides an accurate ranking of oil recovery for each of the stochastic shale realizations. As observed in Fig. 6.8, the cases with stochastic shales have slightly higher recovery than the equivalent homogeneous anisotropic cases (broken line) at higher Kp_eff. It appears that shales have some positive effect on the recovery since they may work as seals to water encroachment. This effect, however, is small. The behavior of the water cut is more correlated to cumulative water injection than to oil recovery. (See Fig. 6.9) This fact is useful since it is possible to predict water cut as a function of cumulative water injection volume. However, it is very important to plot water cut against time as in Fig. 6.3 if one is interested in the variability of its behavior. Plots of production performance against volumetric data such as cumulative production or injection will not provide useful information to estimate the uncertainty in production controlled by pressure constraints.

\subsection{Renormalization of the System}

A theory for obtaining the effective average permeability for a heterogeneous reservoir has been formulated by King [40]. He treated fine grids as a network of electrical resistors and grouped subsets of two by two cells through Kirchoff's law to calculate a 'renormalized' permeability. After several 'generations' of renormalization, the average permeability tended to a stationary value. It is of interest to determine if such a method would work for our model, where shale is completely impermeable and has infinitesimal thickness. One of the shale realizations was descretized into 4 by 4 by 4 coarse grids and the vertical permeability of each coarse grid was computed in the same way as for Kv_eff. Figure 6.10 is the distribution of the resulting 64 values. In contrast to what was obtained by King, this method does not work well in this system. None of the $\mathrm{Kv}$ values is lower than either $\mathrm{Kv}$ _eff or Kp_eff. It is obvious that there is no way of averaging these coarse grids that could result in either Kv_eff or Kp_eff. The problem arises from the way the boundary conditions are specified. Although the effective vertical permeability of the coarse grids in this particular example are higher than what they should be, they could be either higher or lower. The mechanism for the most common case, in which no flow boundaries are applied in the horizontal direction and constant pressure boundaries are applied in the vertical direction, is illustrated in Fig. 6.11. When the no flow boundary condition is imposed on vertical edges, flow paths that goes across these edges to detour around a shale barrier are forbidden. This increases the flow resistance of the coarse grid and thus reduces effective permeability. On the other 

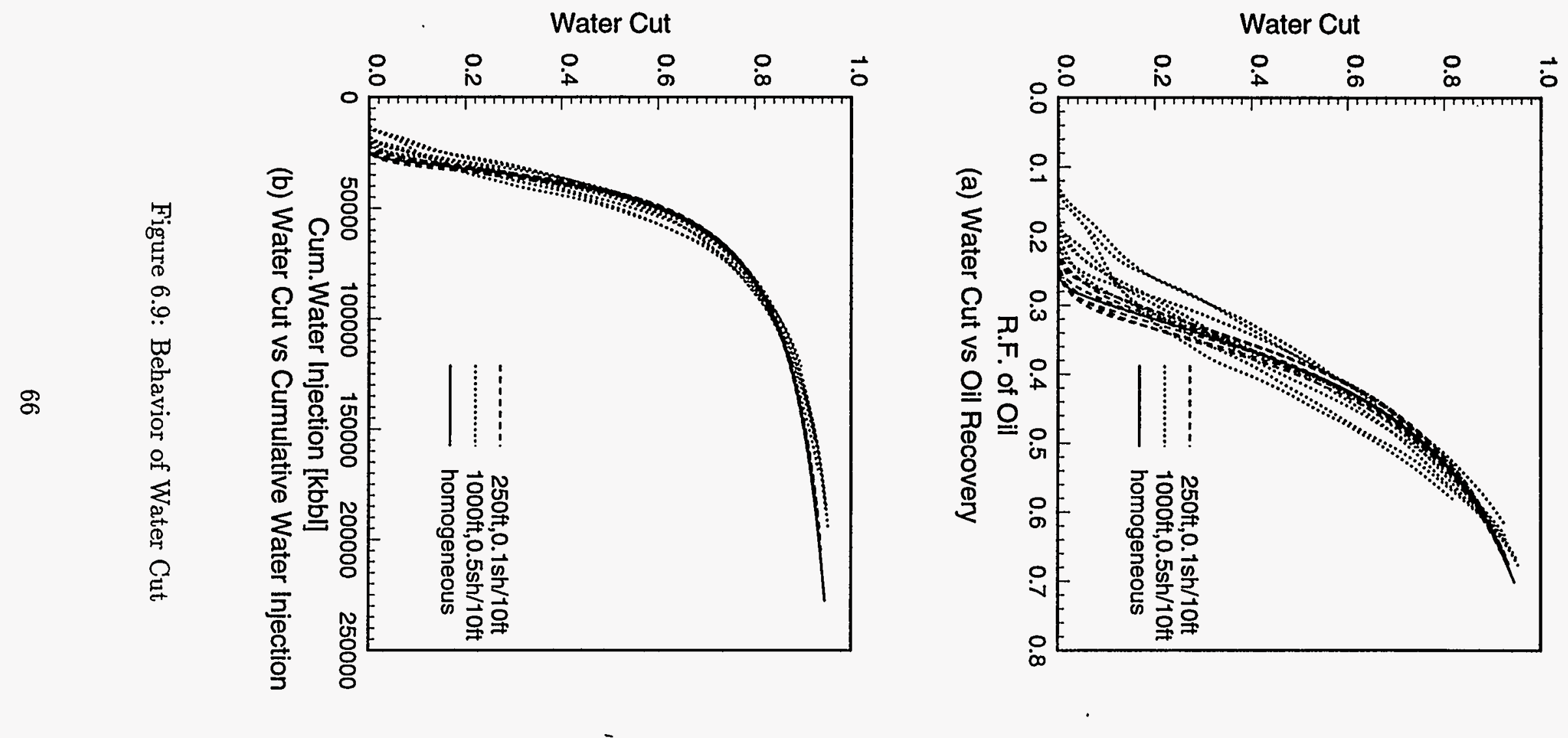


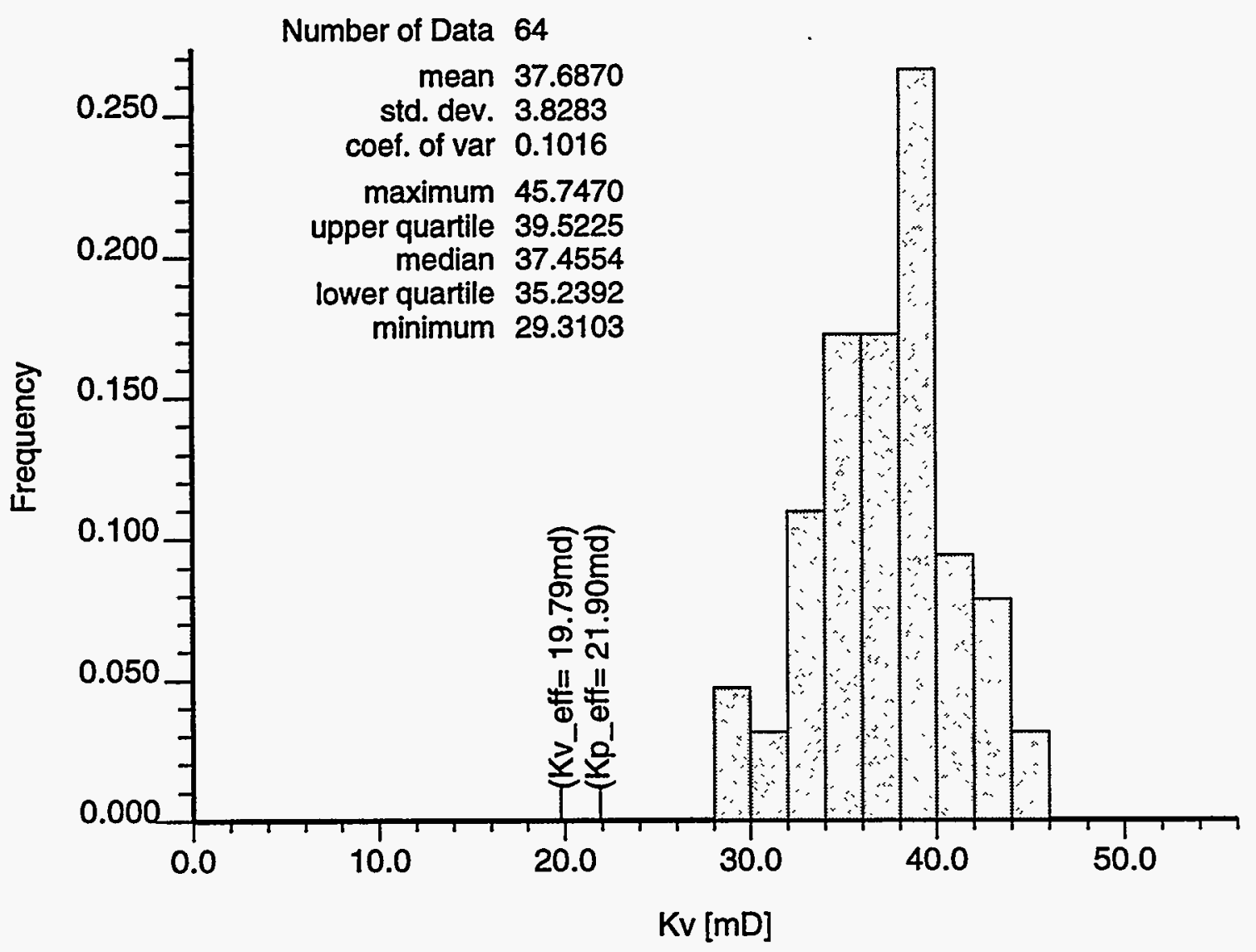

Figure 6.10: Distribution of Coarse Grid Kv 
hand, the constant pressure boundary condition applied to the top and bottom of the coarse grid allows streamtubes to concentrate to an entry point that minimizes the pressure drop across the grid. There is no resistance to the horizontal shift of these streamtubes since the pressure at a boundary is uniform. This results in an increased effective permeability. Since in this particular model, the coarse grids have a much larger boundary on the top and bottom than along the sides, the latter effect is dominant and the effective coarse grid permeability is increased. When the coarse grids are imbedded in a true flow field, the pressure distribution over the top or bottom plane of a coarse grid cell is very nonuniform and a no flow vertical edge will seldom be present. Therefore, if one wishes to obtain the appropriate effective permeability for highly heterogeneous reservoirs, all of the tortuous paths of these streamtubes must be captured. Simple renormalization does not do this. This is the same reason that Kp_eff works better than Kv_eff. The boundary conditions'for the flow problem significantly influence the resulting effective flow property.

\subsection{Conclusions}

1) The production behavior of horizontal wells is strongly affected by the presence of stochastic distributions of discontinuous shales. 2) The effective vertical permeability obtained from linear flow (Kv_eff) fails to correlate the effects of a shale distribution on oil recovery. In order to obtain a representative vertical permeability, it is necessary to honor the boundary conditions so that the model accounts for all of the tortuous flow paths. Simple renormalization techniques do not work because of the inappropriate representation of the boundary conditions. 3) The productivity-based effective vertical permeability (Kp_eff) provides a solution to this problem. It has an excellent correlation with oil recovery, and since the correlation is a monotonically increasing function, it works as a single ranking parameter for the oil recovery from each realization. This means that a probability distribution for oil recovery can be constructed by running flow simulations on only a selected subset of the realizations determined by their rank. 4) A good correlation between cumulative water injection volume and water cut masks its variability. In order to evaluate the uncertainty of production performance under well pressure constraints, it is important to plot the well behavior against time. 


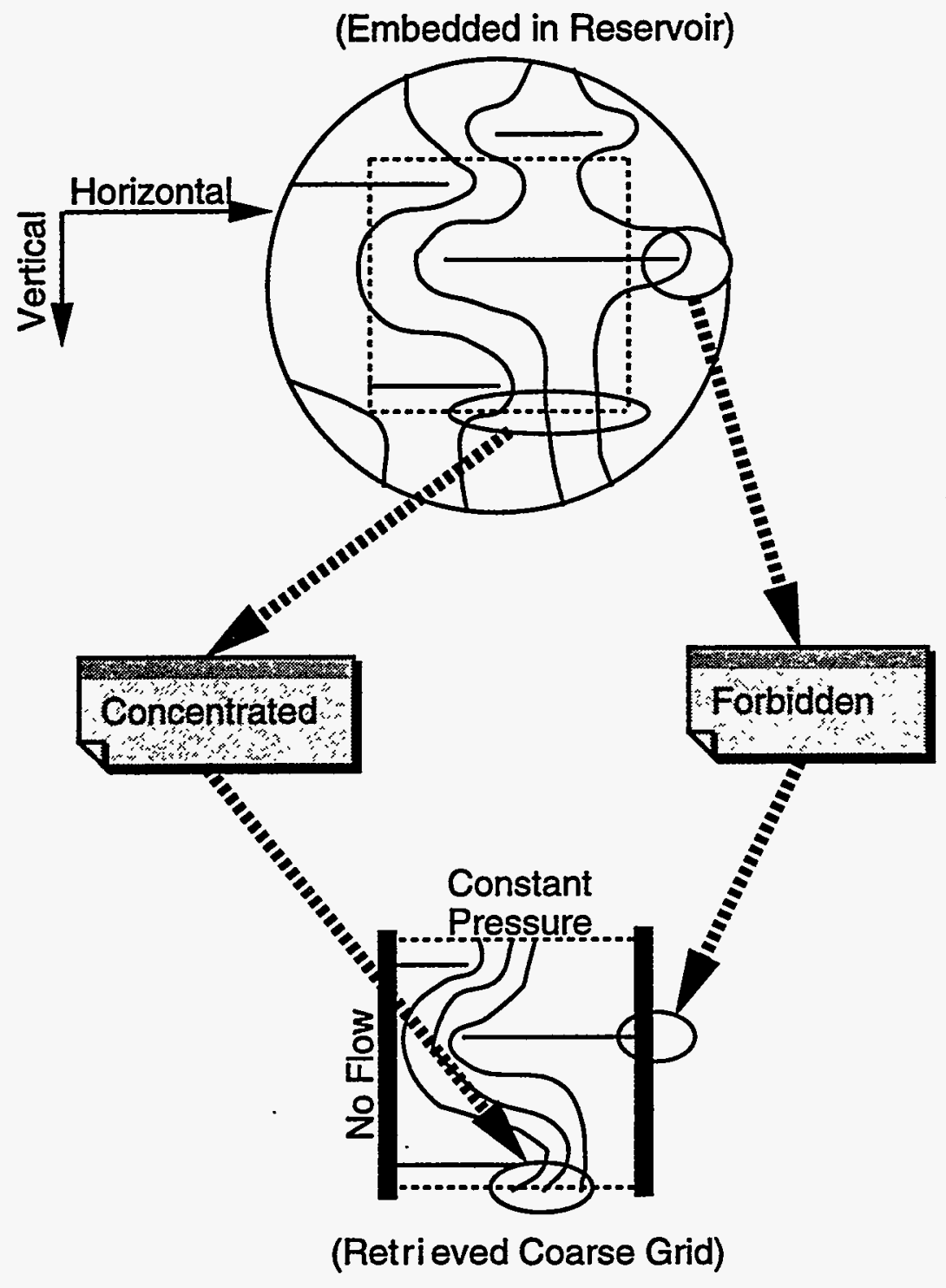

Figure 6.11: Effect of Boundary Conditions on Flow Resistivity 


\section{Analytic Solutions for Coning and Cresting Behavior (Review: Tasks 1 and 3)}

Reservoirs with bottom water or/and gas cap can exhibit coning and cresting behavior. For instance, oil production through a horizontal well underlain by a water zone causes the oil-water interface to deform into a crest shape. The height of the water crest increases as the production rate is increased. At a certain production rate the water crest becomes unstable and water is produced into the well. The production of coned water or gas can reduce oil production significantly. Therefore, it is important to minimize or at least delay coning and cresting. Oil production rates are proportional to the pressure drawdown, and by minimizing drawdown, one may avoid coning problems; but this would also result in reducing the oil production rate. The maximum rate at which oil is produced without production of gas or water is defined as the critical rate.

We expect the cresting behavior in horizontal wells to be much weaker than the coning tendencies in vertical wells. One reason for this difference is the fact that for a vertical well the majority of the pressure drawdown is disposed near the wellbore. In the case of horizontal wells, with their smaller production rates per unit length, the pressure drop is more uniform throughout the reservoir. In a small region near the wellbore, an additional pressure drop is observed but its magnitude is small compared to that around a vertical wellbore. Because of this low pressure drawdown, a substantially higher oil production rate should be possible without exceeding the critical coning rate for horizontal wells.

\subsection{Critical Rates}

There exist at least four analytical methods for quantifying critical rates for horizontal wells. In this section we briefly review these methods, apply them to a base example and compare their predictions.

Giger [41, 42] and Karcher et al. [43] have studied the coning behavior in drainholes and horizontal wells. They have defined two sets of boundary conditions affecting the steady state critical rate $Q_{o, c}$ of an infinite horizontal well in an isotropic formation: 1) edge drive with constant interface height at a specified position and no-flow in the cone and 2) bottom aquifer drive with variable up-flow along a lower boundary at constant pressure. For the first boundary condition, the critical rate for a horizontal well drilled at the top of the reservoir is nearly independent of drainage radius. Giger [41] gives the solution

$$
Q_{o, c}=\frac{k_{h} \Delta \rho g h^{2}}{\mu_{o} B_{o} x_{e}}\left[1-\frac{1}{6}\left(\frac{h}{x_{e}}\right)^{2}\right] L
$$

in which $x_{e}$ is the horizontal spacing longitudinal to the well, $h$ is the reservoir thickness (pay thickness), and $L$ is the length of the horizontal well. Other parameters are defined in the Nomenclature. The assumption of a free surface at a large distance makes the solution invalid for small values of the dimensionless drainage radius. A similar result was derived 
earlier (early Sixties) by Efros [44] in the form of

$$
Q_{o, c}=\frac{k_{h} \Delta \rho g h^{2} L}{\mu_{o} B_{o}\left[x_{e} / 2+\sqrt{x_{e}^{2} / 4+\left(h^{2} / 3\right)}\right]}
$$

These two expressions give very close predictions. Giger [42] and Karcher et al. [43] used the second type of boundary condition to derive the critical rate during a bottom water drive given by

$$
Q_{o, c}=\frac{3 k_{h} \Delta \rho g x_{e}}{2 \mu_{o} B_{o}}\left[\sqrt{1+\frac{16 h^{2}}{3 x_{e}^{2}}}-1\right] L
$$

In another analysis Joshi [22] used Giger's theory, and then assumed that the drainage radius of vertical and horizontal wells are equal, to predict isotropic critical rates. His formulation is given by

$$
Q_{o, c}=Q_{o, c v} \frac{\left[h^{2}-\left(h-l_{h}\right)^{2}\right] \ln \left(r_{e} / r_{w}\right)}{\left[h^{2}-\left(h-l_{v}\right)^{2}\right] \ln \left(r_{e} / \hat{r}_{w}\right)}
$$

where $l_{h}$ is the distance between the horizontal well and the gas/oil interface, $l_{v}$ is the height between the interface and the top end of the completion for a vertical well, $r_{w}$ and $r_{e}$ are the well and drainage area radii respectively, and $\hat{r}_{w}$ is the effective wellbore radius from

$$
\hat{r}_{w}=\frac{r_{e}(L / 2)}{a\left[1+\sqrt{1-[L /(2 a)]^{2}}\right]\left[h /\left(2 r_{w}\right)\right]^{h / L}}
$$

in which $a=(L / 2)\left[0.5+{\sqrt{0.25+\left(2 r_{e} / L\right)^{4}}}^{0.5}\right.$. Finally, $Q_{o, c u}$ is the vertical well critical rate given by

$$
Q_{o, c v}=\frac{\triangle \rho k_{h}\left[h^{2}-\left(h-l_{v}\right)^{2}\right]}{\mu_{o} B_{o} \ln \left(r_{e} / r_{w}\right)}
$$

Chaperon [45] studied the cresting behavior toward a horizontal well in anisotropic formations assuming constant interface elevation at a finite distance. Her approach is based on maintaining static and dynamic equilibrium. The static equilibrium is achieved when the viscous flow potential difference equals that of gravity potential difference. This gives a relationship between distance from the well to the apex of a crest in static equilibrium. But under a perturbation the crest can accelerate toward the well if the changes in buoyancy forces are smaller than viscous forces. The dynamic equilibrium then requires the additional stability condition that perturbations in buoyancy forces must be larger than those from viscous forces. The combination of static equilibrium and stability criteria yields an implicit equation for the critical cone locations. The critical rate based on this method is given by

$$
Q_{o, c}=F \frac{2 \triangle \rho k_{h} h^{2}}{x_{e} \mu_{o} B_{o}} L
$$

The dimensionless function $F$ depends on the parameter $\alpha=\left(x_{e} / 2 h\right) \sqrt{k_{v} / k_{h}}$ which has been given in tabular form in Ref. [45] and also correlated in the form [23] of

$$
F=3.9624955+0.0616438 \alpha-0.00054 \alpha^{2}
$$


The above solution is valid for $1 \leq \alpha<70$ and $x_{e}<4 L$.

When the height of a crest containing immobile water beneath a horizontal well increases, the area open to radial flow is reduced. The oil flow velocity is increased which in turn results in a higher pressure drawdown and an increase in coning tendency. The Chaperon method neglects the flow restrictions due to the immobile water in the crest and therefore should give an overestimate of the critical rate. The conclusions based on this approach can be summarized as: (1) the critical rate for horizontal wells decreases weakly when vertical permeability decreases while the reverse seems to occur in the case of vertical wells (2) horizontal wells generally allow higher critical rates than vertical wells, but this advantage is reduced with an increase in anisotropy.

Recently Guo and Lee [46] have presented another solution based on conformal mapping ideas for the critical oil rate of horizontal wells with water-oil interface cresting. For a fixed oil flow rate and fixed pressure at the outer boundary of the reservoir, the distribution of the pressure in the reservoir is a function of reservoir conductivity which is defined as the ratio of the rock permeability to the viscosity of the flowing fluid. The solution is obtained by mapping (isogonal mapping) the flow region on to a new plane (hodograph plane) in which the velocities act as coordinates. The resulting solution to the critical oil rate for an isotropic reservoir is given by the simple expression

$$
Q_{o, c}=F_{c} \frac{h k \triangle \rho}{\mu_{o} B_{o}} L
$$

where $F_{c}$ is a critical crest height factor given by Fig. 3 in Ref. [46]. $F_{c}$ can be approximated by the fitted equation $\left(0.3 \leq H_{d} \leq 0.85\right)$

$$
F_{c}=7.3497 \times 10^{-5}-1.4129 \times 10^{-4} H_{d}+7.3550 \times 10^{-5} H_{d}^{2}
$$

in which the critical crest height $H_{d}$ is given by the empirical formula [46]

$$
H_{d}=2.31(1.18-0.000246 h)(2.286 \Delta \rho g+0.77)\left(\log k_{x}+8.14\right) \log \left(x_{e} / 2\right)
$$

They have performed sensitivity analyses and found that the critical rate is insensitive to vertical permeability, rock porosity, and water viscosity.

The anisotropic case is dealt with by the change in variables described in Section 3.4. The 2-D anisotropic case with the well in the $y$-direction can be transformed into an equivalent isotropic form with the changes in the $x$ and $z$ coordinates based on using $\bar{k}=\sqrt{k_{x} k_{z}}$. Then Eq. (7.9) becomes

$$
Q_{o, c}=F_{c} \frac{h \bar{k} \Delta \rho}{\mu_{o} B_{o}} L
$$

where the factor $F_{c}$ is still given by Eq. (7.10).

\subsection{Calculation Example}

A numerical example is devised to determine the range of $Q_{o, c}$ predicted by the different methods reviewed above. We consider a reservoir with a gas cap and use the data 
given in the Example (8-7) of Joshi's book [23]. In this example a horizontal well with length $L=1640 \mathrm{ft}$ is drilled $72 \mathrm{ft}$ below the gas-oil contact. The isotropic case is considered with $k_{h}=k_{v}=70 \mathrm{mD}$. For the anisotropic case $k_{v}=0.1 k_{h}$. Other parameters in the example are $x_{e}=2640 \mathrm{ft}, \mu_{o}=0.42 \mathrm{cP}, B_{o}=1.1 \mathrm{RB} / \mathrm{STB}, \Delta \rho=0.48 \mathrm{gm} / \mathrm{cc}, r_{w}=0.328 \mathrm{ft}$, and $r_{e}=1489 \mathrm{ft}$. The predicted critical oil rate for each method is calculated using the corresponding equation in the form of field units (see Ref. [23]). A summary of the results is given in Table 7.1.

Table 7.1: Estimates of Critical Oil Rates for a Single Horizontal Well from Different Methods

\begin{tabular}{||c|c|c||}
\hline \multirow{2}{*}{ Method } & Isotropic & Anisotropic $\left(\frac{k_{u}}{k_{h}}=0.1\right)$ \\
\cline { 2 - 3 } & $Q_{0, c}, \mathrm{STB} /$ day & $Q_{0, c}, \mathrm{STB} /$ day \\
\hline Efros $^{[44]}$ and Giger ${ }^{[41]}$ & $114^{*}$ & - \\
Giger $^{[42]}$ and Karcher et al. ${ }^{[43]}$ & 457 & - \\
Joshi $^{[22,23]}$ & $492^{* *}$ & - \\
Chaperon $^{[45]}$ & 1122 & 985 \\
Guo and Lee & 2759 & 872 \\
\hline
\end{tabular}

Results in Table 7.1 show a large discrepancy among the above methods for prediction of critical oil rates for a single horizontal well. They differ as much as a factor of 24 in the isotropic case. It is surprising to see that the prediction by Guo and Lee's method in the isotropic case is much larger (a factor of more than 2) than the overestimate of the rate expected from the Chaperon method. This is contrary to the claim that flow restrictions due to presence of the cone will reduce critical flow rate in the conformal mapping solution. The anisotropic cases show a reverse trend.

A similar example for a vertical well in the case of a reservoir with bottom water is given in Example (8-1) of Ref. [23]. The critical oil rates are estimated by five different methods. The maximum variation between the extreme pair is of order of about 2 while the other three methods give estimates which differ only up to 25 percent. Therefore, it is apparent that the methods for predicting critical oil rates for vertical wells are more consistent with each other than those for horizontal wells.

*This value is listed as $57 \mathrm{STB} /$ day in Ref. [23]

**This value is calculated to be $470 \mathrm{STB} /$ day in the corrections to Ref. [23] 


\section{Critical Rates and Breakthrough Times in Vertical and Horizontal Wells - Simulation Results (Tasks 1 and 3)}

\subsection{Introduction}

In this chapter, we present simulation results for the coning and cresting critical rates for vertical and horizontal wells. The isotropic case of a gas coning example has been used (based on Example 8-7 of Joshi's book [23]) with the basic reservoir properties listed below. The reservoir has a gas cap with a gas/oil contact $72 \mathrm{ft}$ above a horizontal well of length $L=1640 \mathrm{ft}$. Other parameters are $r=2640 \mathrm{ft}, \mu_{o}=0.42 \mathrm{cP}, B_{o}=1.1 \mathrm{RB} / \mathrm{STB}$, $\Delta \rho=0.48 \mathrm{gm} / \mathrm{cc}, r_{w}=0.328 \mathrm{ft}, r_{e}=1489 \mathrm{ft}$, and $k_{h}=k_{v}=70 \mathrm{mD}$. In the case of vertical wells, simulation results of critical rates are compared with four analytical solutions. The analytical method due to Wheatley [47] agrees with the simulation results very well. The method of Meyer and Garder [48] underestimates the critical coning rates while the Chaperon method [45], which is only applicable to the case with the well at the bottom of the reservoir, overestimates the critical rate. The recently proposed method of Guo and Lee [49] gives rates that are much higher than the simulation results (up to a factor of 14). It also predicts a maximum in the critical rate for a completion penetration fraction of about $1 / 3$. This feature of their method is contrary to the simulation behavior as well as to the solution of the other three analytical methods.

Another important feature of horizontal wells is their ability to delay gas or water breakthrough into the well. Simulation runs are made to compare the breakthrough times of the crest into the horizontal well with the analytical results of Papatzacos et al. [50]. It is shown that horizontal wells can significantly postpone the breakthrough time of gas into the well in comparison with vertical wells.

In the next section we briefly review the Wheatley's method [47] for estimation of critical coning rates for vertical wells. This is followed by presentation of our results. Finally, we draw some conclusions in the last section of the report.

\subsection{Summary of Wheatley's Method}

Wheatley [3] presents an approximate analytical theory for the problem of water coning into a partially penetrating oil well. Figure 8.1 shows the schematic of the problem. The reservoir is taken to be homogeneous and bounded above by a horizontal impermeable barrier extending to the drainage radius $r_{D e}$ from the well. The influx of oil at the drainage radius is assumed to be steady and radially symmetric. The flow region is bounded below by the OWC which is deformed toward the well due to the reduction in pressure near the well. 


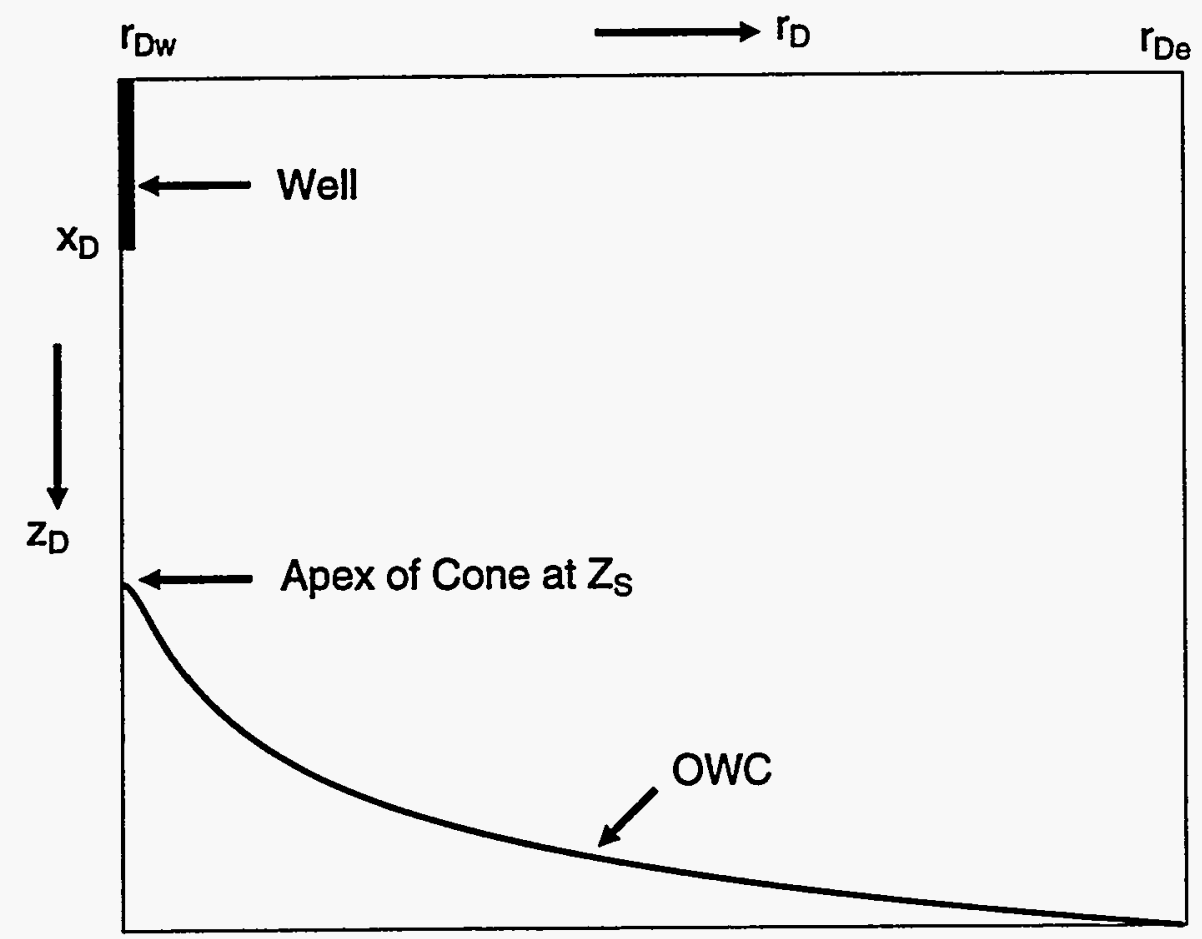

Figure 8.1: Schematic of the Water Coning Problem

Wheatley defined the fluid potential, $\Phi$, in the oil phase, parametrically, as follows:

$$
\Phi=\frac{q}{\left(a X_{D}-b\right)}\left(A_{1}+a A_{2}+b A_{3}-A_{4}\right)
$$

where

$$
\begin{aligned}
A_{1}\left(r_{D}, Z_{D}\right) & =2 \ln r_{D}-g\left(r_{D}, Z_{D}-Y_{D}\right)+g\left(r_{D}, Z_{D}+Y_{D}\right) \\
A_{2}\left(r_{D}, Z_{D}\right) & =g\left(r_{D}, Z_{D}-X_{D}\right)-g\left(r_{D}, Z_{D}+X_{D}\right) \\
A_{3}\left(r_{D}, Z_{D}\right) & =f^{-1}\left(r_{D}, Z_{D}-X_{D}\right)+f^{-1}\left(r_{D}, Z_{D}+X_{D}\right) \\
A_{4}\left(r_{D e}, 1\right) & =A_{1}\left(r_{D e}, 1\right)+a A_{2}\left(r_{D e}, 1\right)+b A_{3}\left(r_{D e}, 1\right)
\end{aligned}
$$

and

$$
\begin{aligned}
& f(r, z)=\left(r^{2}+z^{2}\right)^{1 / 2} \\
& g(r, z)=\ln [z+f(r, z)]
\end{aligned}
$$

The dimensionless parameters and variables are the fractional completion penetration; $X_{D}=x / h$, the dimensionless radius $r_{D}=r\left(k_{v} / k_{h}\right)^{1 / 2} / h$, and the dimensionless depth $Z_{D}=z / h . h$ is the thickness of oil column and $k_{v}$ and $k_{h}$ are the vertical and horizontal permeabilities. $Y_{D}$ is a dimensionless depth, to be determined for the start of a semi-infinite line source on the well axis.

Construction of the potential function in Eq. (8.1) is based on an ingenious combination of line and point source terms to satisfy the Laplace equation. In addition, the potential 
function in Eq. (8.1) satisfies exactly or nearly exactly all the boundary conditions of the problem:

(i) $\Phi=0$ at $r_{D e}$ and $Z_{D}=1$ and is approximately zero at $r_{D e}$ for $0<Z_{D}<1$.

(ii) $\Phi=-\Delta p$, the maximum pressure drop across the reservoir at $r_{D w}$ and $Z_{D}=0$. $\Phi$ is approximately constant at $r_{D w}$ for $0<Z_{D} \leq X_{D}$.

(iii) $\Phi=-\Delta \rho g h(1-z)$ on the OWC which is actually the streamline.

(iv) $\partial \Phi / \partial z=0$ on $Z_{D}=0$ and $\partial \Phi / \partial r=0$ on $r_{D}=0$ and $Z_{D}>X_{D}$

The line source strength $q$ can be computed from

$$
q=q_{D C} \Delta \rho g h\left(a X_{D}-b\right)
$$

once $q_{D C}, a, b$ and $Y_{D}$ have been determined. The unknown parameters are $q_{D C}$, the dimensionless critical rate; $a$ and $b$, relative strengths of line and point sources, respectively; $Y_{D}$, the dimensionless depth of the line source; and $Z_{S}$, the dimensionless depth to the apex of the cone.

Wheatley in Ref. [47] has proposed an iterative procedure to determine these unknowns along with the critical oil rate. His procedure has been employed in obtaining the relevant results reported in the next section. A step by step outline of his procedure is given below:

1. Choose an initial guess value of $Z_{S}$ slightly less than one and set $Y_{D}=1$.

2. Calculate $a, b$ and $Y_{D}$ by iteration from:

$$
\begin{aligned}
a X_{D}-b & =\left(r_{D e}-Y_{D}\right) /\left(r_{D e}-1\right) \\
b / r_{D w} & =a \ln \left(r_{D w} / X_{D}\right)-\ln \left(1-X_{D}^{2} / Y_{D}^{2}\right) \\
Y_{D}^{2} & =Z_{S}^{2}+\frac{Z_{S}\left(Z_{S}^{2}-X_{D}^{2}\right)^{2}}{\left(a X_{D}-b\right)\left(Z_{S}^{2}-X_{D}^{2}\right)-2 b X_{D}^{2}}
\end{aligned}
$$

3. Calculate the dimensionless rate from

$$
q_{D}=\left(Z_{S}-1\right) /\left(A_{1}+a A_{2}+b A_{3}-A_{4}\right)
$$

where $A_{1}, A_{2}$ and $A_{3}$ are evaluated from Eqs. (8.2)-(8.7) at $r_{D}=0$, and $Z_{D}=Z_{S}$.

4. Reduce the estimate for $Z_{S}$ and recompute $a, b, Y_{D}$ and $q_{D}$.

5. Repeat step 4 until a maximum in $q_{D}$ is obtained, above which self-consistent values of $a, b, Y_{D}$ and $Z_{S}$ cannot be obtained.

6. Reduce $Z_{S}$, keeping $a, b$ and $Y_{D}$ fixed, and recompute $q_{D}$ using Eq. (8.12). Note that $A_{1}, A_{2}, A_{3}$ are functions of $Z_{S}$.

7. Repeat step 6, until a new maximum $q_{D}=q_{D C}$ is obtained where $Z_{S}=Z_{S \min }$. 
8. Finally, compute the critical rate from

$$
q_{c}=6.145 \times 10^{-3} h^{2} k_{h} \Delta \rho q_{D C} / \mu_{o} B_{o}
$$

where $q_{c}$ is the critical oil production rate in STB/Day, $h$ is oil zone thickness $(\mathrm{ft}), k_{h}$ is horizontal permeability $(\mathrm{mD}), \Delta \rho$ is the density difference $(\mathrm{gm} / \mathrm{cc}), \mu_{o}$ is oil viscosity $(\mathrm{cP})$ and $B_{0}$ is the oil formation volume factor (RB/STB). The same procedure can be used for the gas coning problem (as used here) by taking the origin of $Z_{D}$ at the bottom of the oil zone. The resulting potential function has the same properties given above where $\triangle \rho$ in Eq. (8.8) becomes $\Delta \rho_{\text {og }}$.

This section is divided into two parts. In the first part we report and discuss our results for the critical coning and cresting rates. Results on breakthrough times and their discussion are presented in the second part.

\subsection{Critical Rates}

The analytical solutions for coning and cresting critical rates are all based on the assumption of steady-state condition among other assumptions. Most commercial black oil simulators, like Eclipse used here, do not provide the user with an easy option of specifying constant potential at the outer lateral boundaries of the reservoir. To set up such a boundary condition, we have used oil injectors in the oil zone and gas injectors in the gas cap with specified bottom hole pressure controls to eventually achieve the steady-state condition in the reservoir. Care has to be taken in selecting a fine grid for a 3D-model. Advantage can be taken of symmetry which allows one quarter of a square pattern area to be studied. It is also desirable to select relative permeabilities which will help development of a sharp interface. Determining a critical rate by simulation involves a number of iterative runs in which the oil production rate is varied until a maximum rate is found for which the gas/oil interface eventually reaches steady-state before breaking through into the well. Sometimes breakthrough occurs after a very long period.

The simulation results for vertical wells are presented first. The same reservoir properties and parameters as those given above are used for a gas coning case. Figure 8.2 shows the variation of the critical rate with the completion penetration fraction $X_{D}$. It also compares the simulation results for critical coning rates with the analytical results due to Wheatley [47], Meyer and Garder [48], Chaperon [45] and Guo and Lee [49]. The coning critical rates from simulations agree with the predictions of the Wheatley's method very well (within $5 \%$ difference). The Meyer and Garder method which is known in the literature to underestimate critical rates does the same for this example problem. Chaperon's equation which is only applicable to the case where well is at the bottom of the reservoir, overestimates the simulation result for that well location by $28 \%$. The recently proposed model of Guo and Lee [49] gives results much higher (up to a factor of 14) than the simulation results. In addition, it gives an optimum completion penetration fraction of about $1 / 3$ where all the other methods and the simulations predict a value of zero for the optimum completion penetration fraction (assuming that constant total well rate is possible).

A similar simulation procedure as described above is employed in determining the 


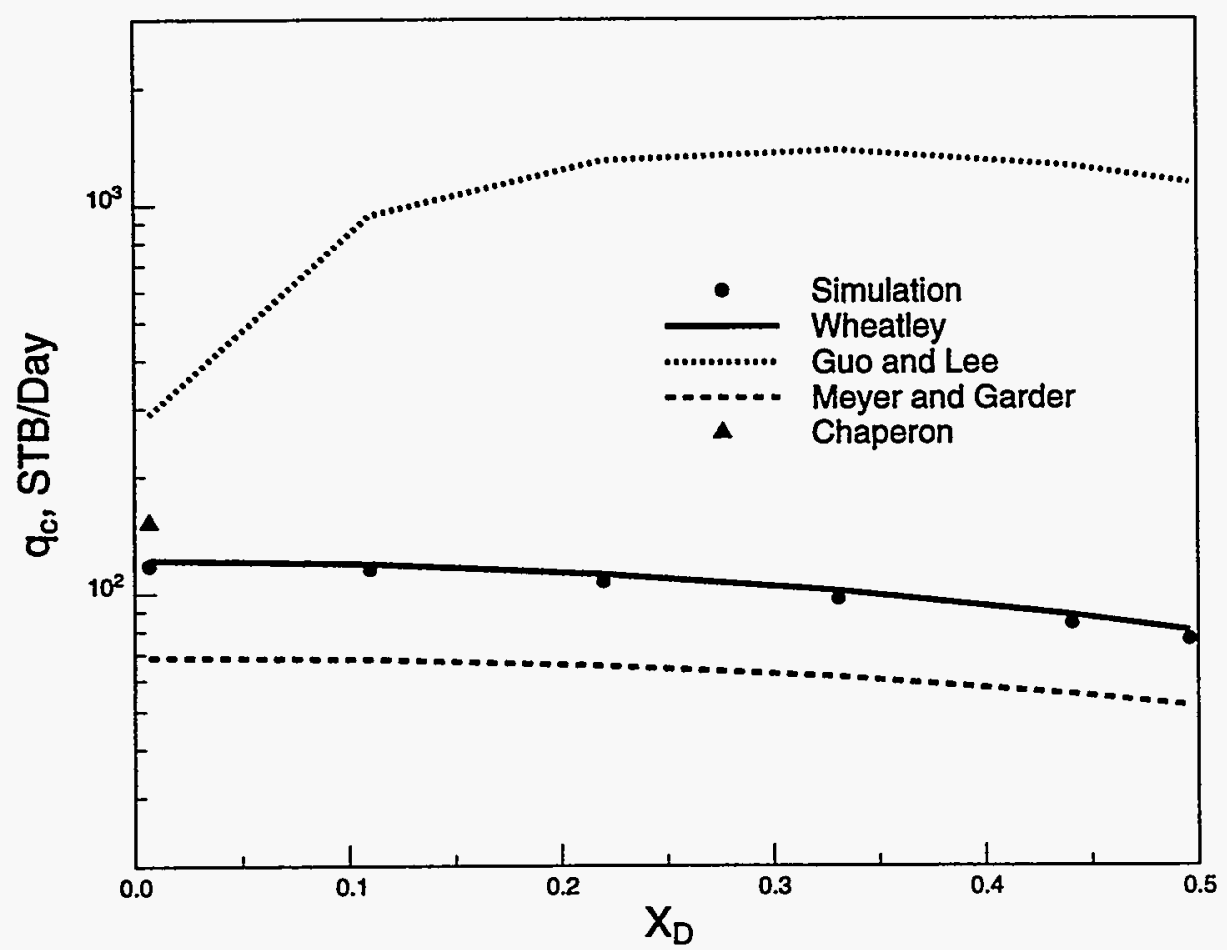

Figure 8.2: Comparison of Critical Rates between Simulation and Analytical Solutions for the Vertical Well at Various Completion Penetration Fractions

critical rate for an infinite horizontal well, using a $2 \mathrm{D}$ x-z grid. Figure 8.3 displays a comparison between critical rates for vertical and horizontal wells. It is assumed that the completion interval of the vertical well is to the same height as the position of the horizontal well (with fixed length of $1640 \mathrm{ft}$ ). The results indicate a marginally higher critical rate for the horizontal well (about $25 \%$ higher). This seems to be a rather modest improvement over the vertical well which might not justify the use of horizontal wells with their higher drilling costs in a field application. Figure 8.4 shows the effect of the reservoir horizontal dimension $x_{e}$ on the critical rate of the horizontal well. These carefully executed simulations show a sharp decrease in the critical cresting rate from high critical rates at close spacing followed by a long slowly declining tail as the spacing increases. Note that the popular Joshi's method [22] overestimates the simulation results by a factor of about 2.5. In fact, for an infinite reservoir there is no critical rate and the true steady state condition is only possible for a zero rate (see Papatzacos et al. [50] and the references therein). This is consistent with the slow decrease of the critical rate toward zero as shown in Fig. 8.4. It is known that the interface behaves like a stretched elastic band so that when the anchor points at the reservoir boundary are far enough apart, the band readily deforms into the horizontal well. We will show in the next part that the major impact of horizontal wells comes from their ability to postpone the breakthrough of gas into the well. 


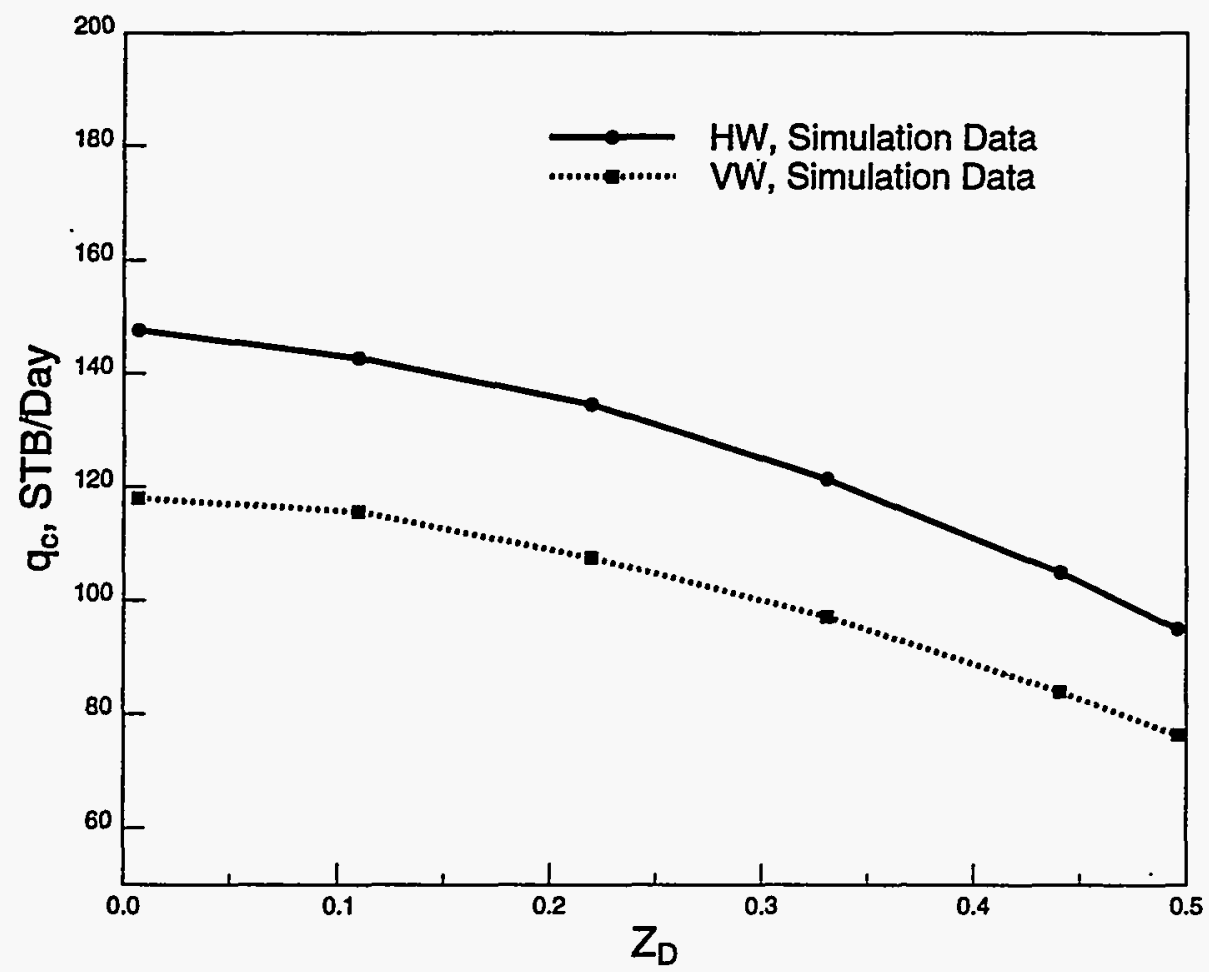

Figure 8.3: Comparison of Critical Rates of Horizontal and Vertical Wells at Various Dimensionless Depths from Simulations

\subsection{Breakthrough Times}

Papatzacos et al. [50] report semianalytical solutions for cone breakthrough times for horizontal wells. Their solutions are derived by a moving boundary method with assumption of either constant pressure or gravity equilibrium in the cone. Furthermore their model gives an asymptotic time for wells with infinite spacing. We only give the final results of that work here. Details can be found in Ref. [50] and the references therein. Two types of conditions are used at the moving crest interface. One is the assumption of constant pressure at the moving boundary which results in the breakthrough time given by

$$
t_{B t D}=1 / 6 q_{D}
$$

where the dimensionless oil rate and time are defined in consistent units by

$$
\begin{aligned}
q_{D} & =\frac{\mu_{o} B_{o}}{2 \pi \sqrt{k_{v} k_{h}} h \Delta \rho g} q_{o} \\
t_{B t D} & =\frac{\Delta \rho g k_{v}}{h \phi \mu_{o}} t
\end{aligned}
$$

The second condition is the assumption of vertical equilibrium in the crest. The solution has been determined by the method of fast Fourier transform (FFT). For $q_{D}>0.4$, the authors have been able to close the iteration procedure and explicitly find

$$
t_{B t D}=1-\left(3 q_{D}-1\right) \ln \left[3 q_{D} /\left(3 q_{D}-1\right)\right]
$$




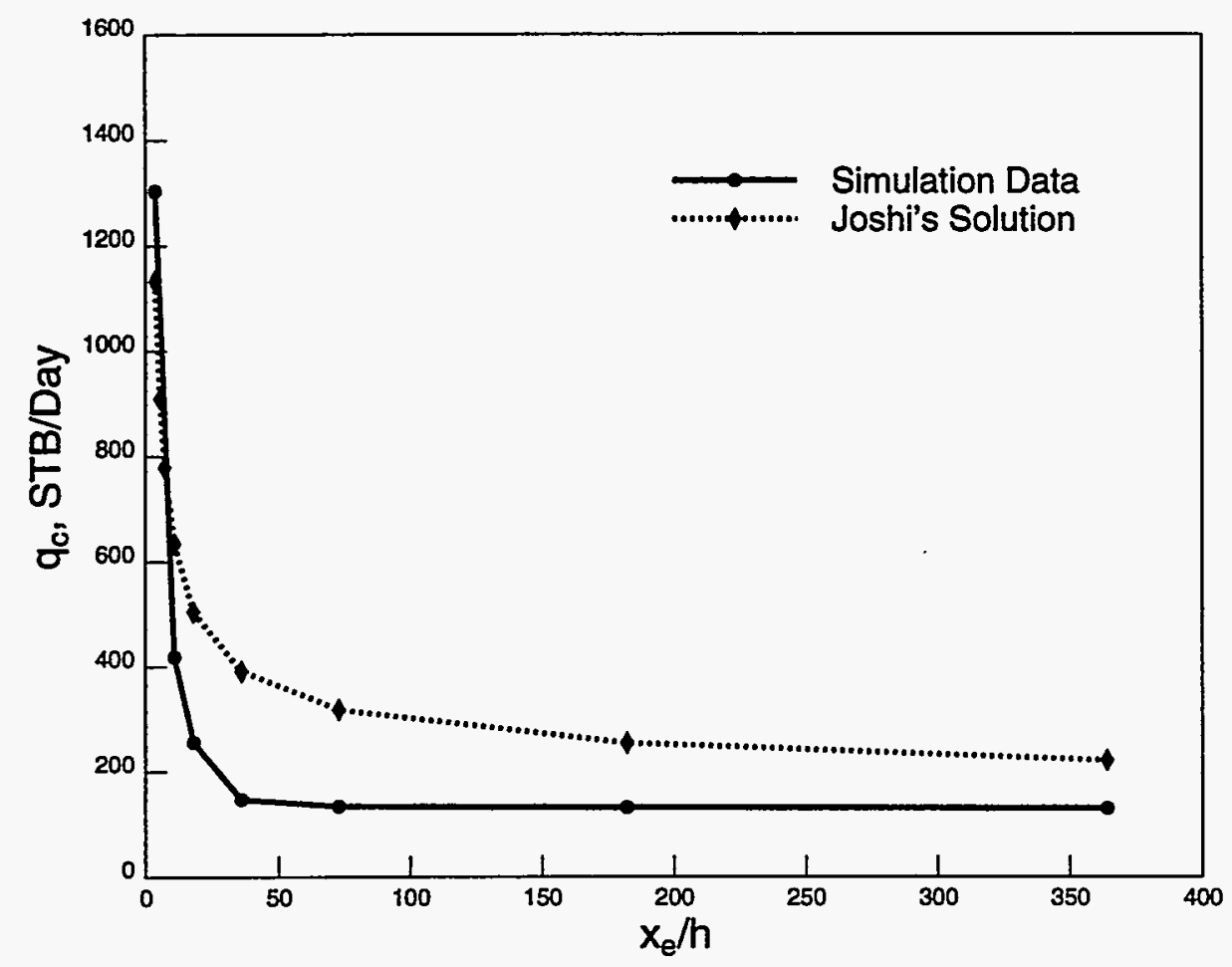

Figure 8.4: Variation of the Critical Rate with the Horizontal Dimension of the Reservoir for the Horizontal Well

For large values of $q_{D}$, Eq. (8.14) becomes equal to Eq. (8.16). For values of $q_{D} \leq 1 / 3$, the FFT solution should be used. A simpler approach is to instead use a quadratic fit to the FFT solution given by [50]

$$
\ln \left(t_{B t D}\right)=-1.7179-1.1633 U+0.16308 U^{2}-0.046508 U^{3}
$$

where $U=\ln \left(q_{D}\right)$.

Figure 8.5 displays our simulation results for breakthrough times for a horizontal well located at the bottom of the reservoir along with the results for a horizontal well $8 \mathrm{ft}$ above the bottom of the reservoir. In both cases the distance between well and the gas/oil interface is fixed at $72 \mathrm{ft}$. At $t_{B t D}=2.0$ in this problem, the corresponding real time is 252 days, and $q_{D}=1.0$ is equivalent to a rate of $13715 \mathrm{STB} /$ Day. The predictions of the semianalytical results discussed above are also shown. Simulation results are closer to the predictions based on the gravity equilibrium condition in Eq. (8.16) for values of $q_{D}<0.3$. Sensitivity of the location of the well is more pronounced for lower flow rates as shown in the Figure.

Earlier in this report we found a marginally higher critical rate for the horizontal well in comparison with the vertical well for our example problem. The important practical factor is not how much higher the critical rate of horizontal wells is relative to those of vertical wells, but rather how much longer a reservoir can be produced without breakthrough of a cone using horizontal wells in comparison with vertical wells. To address this issue we produce the reservoir at supercritical rates by horizontal and vertical wells and determine the breakthrough times. 


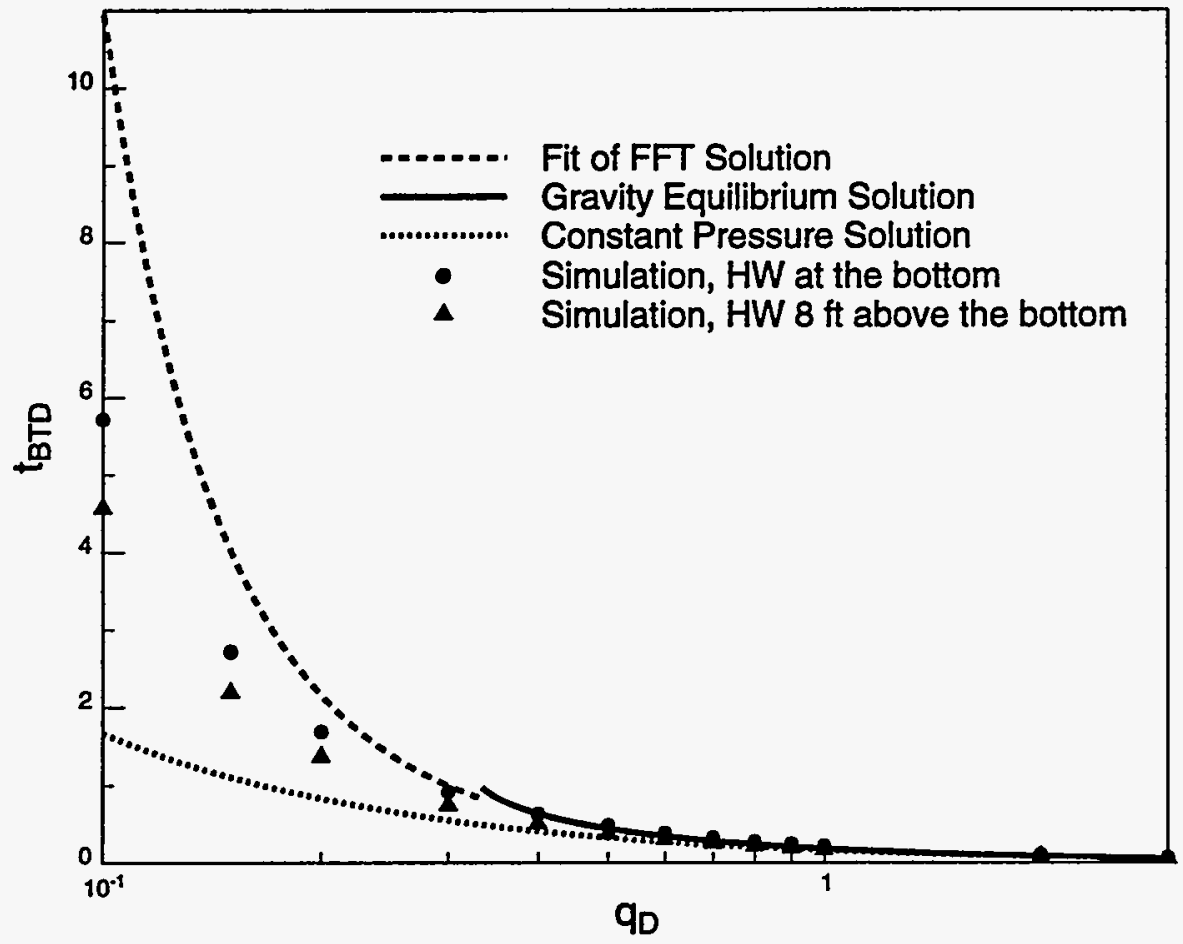

Figure 8.5: Comparison of Breakthrough Times between Simulation and Solutions of Ref. [50] for Horizontal Wells

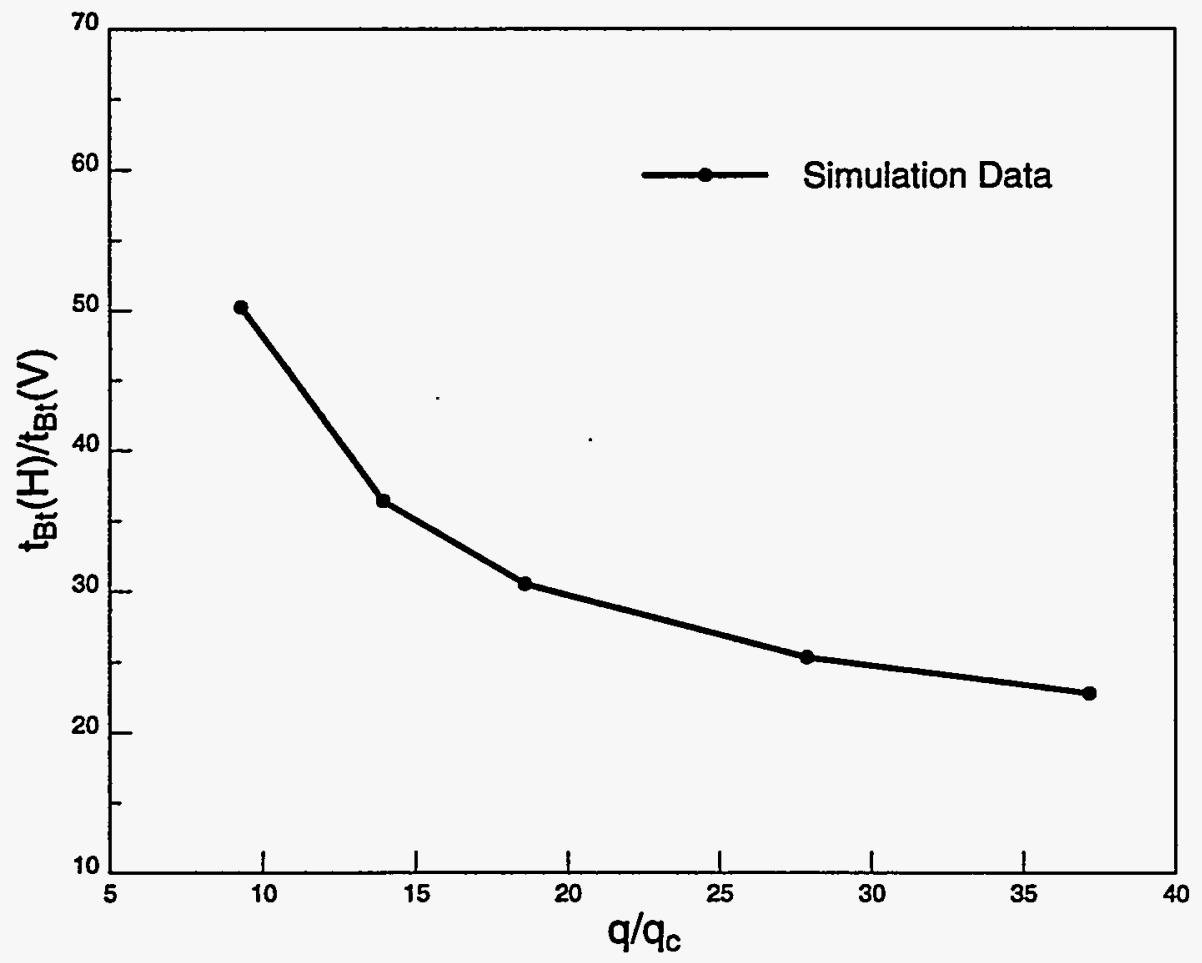

Figure 8.6: Comparison of Breakthrough Times for Horizontal and Vertical Wells at Various Supercritical Rates from Simulations 
We have already estimated the critical coning and cresting rates from the simulations. We may then produce the reservoir at a higher rate obtained by a fixed factor increase in the corresponding critical rates for horizontal and vertical wells respectively. Results for the wells at the bottom of the reservoir are shown in Fig. 8.6, where a significant advantage of horizontal wells in postponing the breakthrough of gas into the well is clearly observed. For example, a factor of about 50 is obtained in extending the breakthrough time with the horizontal well producing at a rate of 9.3 times of its critical rate. In this case, gas breakthrough occurs at 721 days for the horizontal well while it only takes 15 days for the breakthrough in the vertical well at the same relative flow rate condition. This implies a hypothetical condition of a vertical well of almost zero length flowing at 9.3 times its critical rate.

\subsection{Conclusions}

We may draw a number of conclusions from the results presented in this report:

1. The analytical solution of Wheatley [47] appears to give accurate values of the critical coning rates for vertical wells. For the gas coning example studied here, the Wheatley solutions for the critical rates are within $5 \%$ of the corresponding simulation results.

2. The analytical solutions for the critical cresting rates for horizontal wells give a wide range of predictions. The Joshi's method [22] is less restrictive on the location of the horizontal well and although it overestimated the simulation results by as much as a factor of 2.5 , it might be regarded as the preferred method over the other analytical solutions.

3. The solutions of Guo and Lee for both horizontal[46] and vertical wells [49] predict much higher critical rates than the simulation results and the other analytical methods. For instance, their method predicts a critical rate of $2759 \mathrm{STB} /$ Day for our base problem with the horizontal well at the bottom while the simulation gives a critical rate of 148 STB/Day.

4. The important cresting property for horizontal wells is not so much their critical rates, but rather their delayed breakthrough time. This useful trait of horizontal wells becomes more significant as the well spacing becomes longer. 


\section{Uncertainties Caused by Pressure Drop in the Well (Review: Task 3)}

The single-phase in-flow equation given in Eq. (3.1) applies to an infinite well with $P_{w}$ constant. Its application to a finite well requires the determination of self-consistent values of pressure $P_{w}$ inside the well circumference. $P_{w}$ is a function of position along the well which must be evaluated from wellbore pressure drop equations.

\subsection{Single-Phase Flow}

The total pressure drop in single phase flow may be expressed as the sum of three terms associated with friction, momentum changes, and head changes, in the form

$$
\Delta P=\frac{f L \rho \bar{V}^{2}}{r_{w}}+\frac{1}{2} \rho \Delta\left(\bar{V}^{2}\right)+\rho g \Delta h
$$

The most important term is that due to friction in which the Fanning friction factor for laminar flow $(R e<2000)$ is given by

$$
f=\frac{16}{R e}
$$

with the Reynolds number $R e=2 \bar{V} \rho r_{w} / \mu$, but for turbulent flow (Re $\left.>4000\right)$, Haaland's correlation applies:

$$
1 / \sqrt{f}=-3.6 \log \left[\frac{6.9}{R e}+\left(\frac{\epsilon}{7.4 r_{w}}\right)^{10 / 9}\right]
$$

Linear interpolation can be used in the range $2000 \leq R e \leq 4000$. The difficulty in Eq. (9.3) is to select the "effective" absolute roughness $\epsilon$ to use with a given completion design.

The momentum term is often ignored. It becomes marginally more important when it is recognized that the radial inflow of fluid through the perforations has to be accelerated to the core fluid velocity. If $q_{1}$ and $q_{2}$ are the volumetric flow rates at the inlet and outlet ends of a pipe (so that $q_{2}-q_{1}$ is injected radially), then the corresponding momentum term is $\rho\left(q_{2}^{2}-q_{1}^{2}\right) / 2 \pi^{2} r_{w}^{4}$. For a horizontal well of length $2000 \mathrm{ft}, \epsilon=10^{-4} \mathrm{ft}$ and diameter of 6.18 inches, flowing at $10,000 \mathrm{bbls} /$ day with an oil viscosity of $3 \mathrm{cP}$ and $\rho=50 \mathrm{lbm} / \mathrm{ft}^{3}$, the frictional pressure drop is $4.55 \mathrm{psi}$, and the momentum contribution is $0.03 \mathrm{psi}$.

Under single-phase flow conditions, the frictional pressure drop at moderate flow rates along the inside of the well will be quite small provided the completion design is relatively simple; e.g., for a cased hole with normal perforations. In these circumstances treatment of the well as having infinite hydraulic conductivity might be expected to be appropriate. However, in an interesting analysis, Ozkan et al. [51] have shown that even under singlephase flow, the well-bore pressure drop can exert significant influence on well productivity at high flow rates, depending on the assumed relative roughness, $\epsilon_{D}=\epsilon / 2 r_{w}$. Fig. 9.1 reproduced from their paper indicates a significant range of productivity variations for a field case. A value of $\epsilon_{D}=10^{-4}$ is often assumed to apply to smooth steel pipes; however, it is unclear what will be the magnitude of increase due to the perforations and the transverse 
in-flow which will disturb the boundary layer close to the pipe wall. If slotted liners with screens, or open-hole completions are used, considerably larger values of $\epsilon_{D}$ would apply. For an open-hole completion, the continuous radial in-flow might lift the boundary layer away from a rough sandface, and thereby ameliorate the large surface roughness in this case. Ozkan et al. also find that for long thin closed reservoirs (for which horizontal wells are particularly appropriate), the pseudo-steady state results cause significant reductions in productivity from the strict steady-state formulae; e.g., a Peaceman-type equation.

Production Rate $q$, (bbl/d)

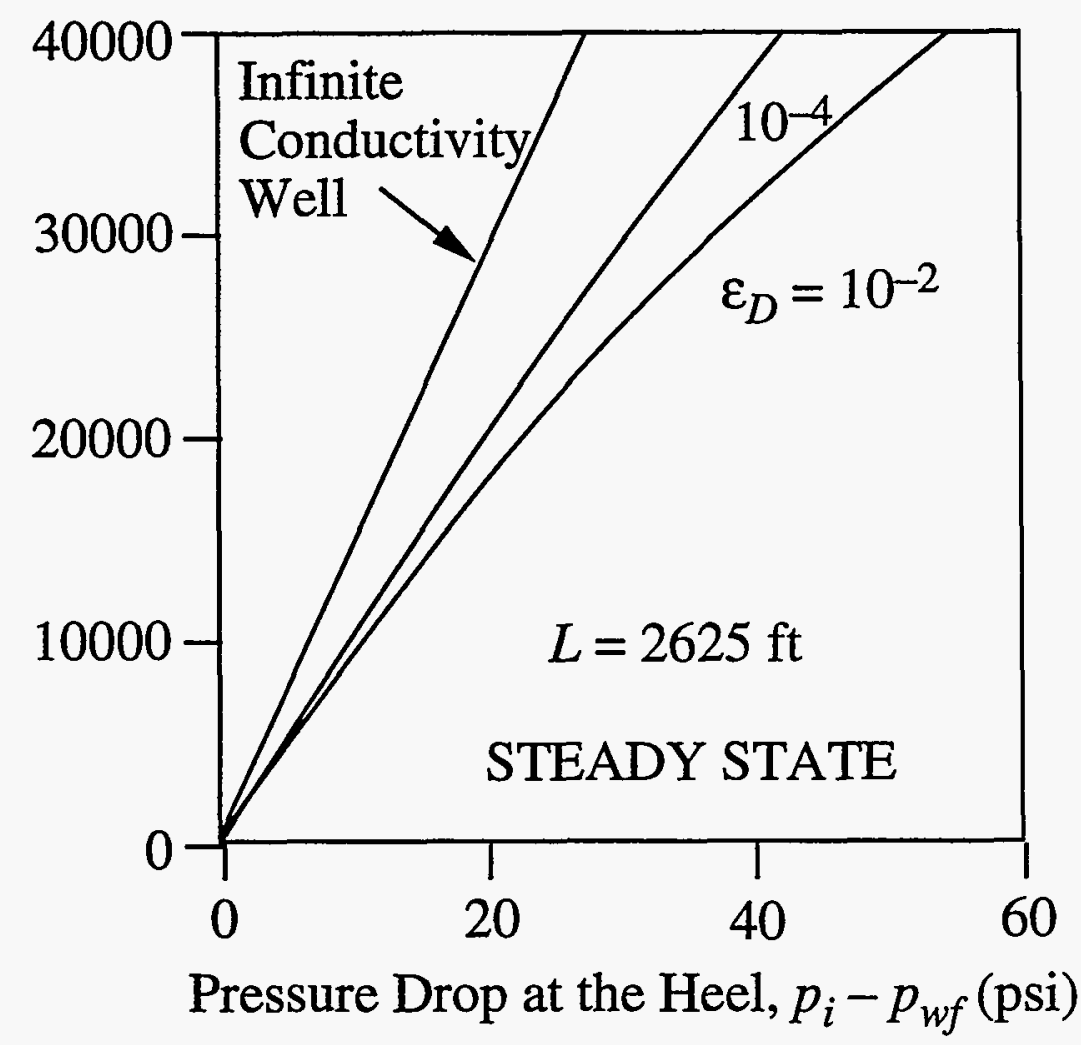

Figure 9.1: Effect of Relative Surface Roughness on the Productivity of the Well (Field Example)[51]

\subsection{Two-Phase Flow Behavior}

When gas or water breakthrough occurs into the well, the pressure drop increases rapidly as the volume fraction of the second phase increases. The effects of gas breakthrough are illustrated in Fig. 9.2. Prior to drawdown of the gas/oil contact into the well, as shown in Fig. 9.2a, the single-phase pressure drop along the length of the wellbore is modest, and causes the drawdown near the entry end to be slightly larger than that occurring near the 
closed end. The larger drawdown near the entry pulls the gas/oil contact into the well at that end, as shown in Fig. 9.2b. The generation of a two-phase state in the entry section of the well now increases the drawdown effect at this end, which then more strongly drives the two-phase fluid into the well. Thus having an accurate procedure for estimating two-phase pressure drops in a long horizontal well can become important.

It is possible to retain Eq. (9.1) provided "effective" two-phase densities and viscosities are used in the definition of Reynolds number, and the flow is assumed to be homogeneous (i.e., no slip between gas and oil phases). There are a number of possible recipes for choosing the effective homogeneous properties. Beattie and Whalley [52] have recommended the following equations:

$$
\begin{gathered}
\frac{1}{\hat{\rho}}=\frac{\alpha_{g}}{\rho_{g}}+\frac{1-\alpha_{g}}{\rho_{o}} \\
\hat{\mu}=\mu_{o}(1-\beta)(1+2.5 \beta)+\mu_{g} \beta
\end{gathered}
$$

where

$$
\beta=\frac{\rho_{o} \alpha_{g}}{\rho_{o} \alpha_{g}+\rho_{g}\left(1-\alpha_{g}\right)}
$$

and $\alpha_{g}$ is the gas volume fraction in the well. These authors claim this method gives good pressure drop predictions for data on horizontal pipes contained in their extensive HTFS library. However, the assumption of homogeneous flow is not usually valid, with there being significant hold-up of the liquid phase. The hold-up behavior varies significantly between flow in pipes sloping slightly upward, compared with those tilted slightly downwards. There is consequently a number of correlations which have been developed to express two-phase friction losses with slip in normal pipe flows, all without radial in-flow. These correlations attempt to recognize the effects of different flow patterns such as segregated, wavy, bubble, mist, slug, annular, churn flow, etc.

The vast amount of literature available on multiphase flow in pipes generally relates to stabilized conditions. This means that measurements have to be made in long sections of pipe, away from entrance and exit regions. Hence the applicability of standard two-phase flow correlations near to the entrance region or end of horizontal wells is subject to question. Furthermore, the perpendicular in-flow from the reservoir occurs at a variety of positions along the well (depending on type of completion), and this will significantly perturb the steady state flow patterns which would otherwise occur in normal pipe flow. The resulting increases in chaotic and turbulent conditions might be expected to cause larger pressure drops.

In addition to the uncertainty of the applicability of two-phase flow correlations to the horizontal well problems, there is also considerable uncertainty about the accuracy of these correlations even for standard pipe flow calculations. Furthermore, computational difficulties arise because many of the well known correlations have sharp discontinuities across flow pattern boundaries. Possible differences in flow pattern predictions by different methods are illustrated in Figs. 9.3 and 9.4. Figure 9.3 shows flow pattern transitions predicted by a general mechanistic model now under development at Stanford University. This model is an extension of the work of Barnea [53]. The flow pattern predictions by this method are sensitive to changes in the angle of inclination and fluid properties, with significant differences between up-flow and down-flow in tilted pipes. A brief review of the model 
and further results are given in chapter 12. Figure 9.4 shows the flow pattern transitions predicted ffor the same case by a modification of the Beggs and Brill method given in Brown [54]. Significant differences in the location of transition boundaries are seen fom the log scales of the axes. Furthermore, The Beggs and Brill method uses the same flow pattern prediction method for all angles of inclination. A consequence of this is that pressure drop predictions for horizontal pipes can be easily in error by $\pm 20 \%$ and the errors can be even larger for inclined pipes. Combining this uncertainty with the added effects of perpendicular in-flow from perforations, the pressure drop at high GOR or WOR for horizontal wells could be underestimated by more than $100 \%$.

Some example calculations have been run to illustrate some of the uncertainties involved. The example problem is for a horizontal well of length 2,000 feet flowing oil at 10,000 $\mathrm{bbls} /$ day with a GOR of $10,000 \mathrm{scf} / \mathrm{bbl}$. The average void fraction is 0.9 . (Only a modest fraction of this GOR is associated with gas in solution). The oil viscosity is $3.0 \mathrm{cP}$. We show results for both a smooth pipe and for a rough pipe with internal diameter 6.18 inches and assuming a roughness for a slotted liner of 0.01 feet. The calculations were undertaken using the following two-phase pressure drop correlations available in the ASA software [55]: Beggs and Brill [5], Dukler, Wicks, and Cleveland [56], and Mandhane, Gregory and Aziz [57], and the homogeneous flow correlation due to Beattie and Whalley [52]. For this purpose it was assumed that all the flow was introduced into the well at the upstream end, rather than distributed as radial in-flow along its length. The results are summarized in Table 9.1. Besides there being a large spread between the correlations, it is noticeable that there are large changes due to effective roughness in the two-phase regime. Even for the smooth pipe results, there are significant pressure drops involved which will affect the well performance. Neglecting flow pattern perturbations, for distributed in-flow we might expect rather less than 0.5 times the pressure drops indicated here for the case of all the injection at the entrance end.

Table 9.1: Estimates of Pressure Drops for the Example Calculation Using Different Correlations

\begin{tabular}{|l|c|c|}
\hline Correlation & DP-smooth, psi & DP-rough, psi \\
\hline Beggs and Brill ${ }^{[5]}$ & 125 & $596^{*}$ \\
Dukler et al. ${ }^{[56]}$ & 197 & $1029^{*}$ \\
Mandhane et al. ${ }^{[57]}$ & 57 & 372 \\
Beattie and Whalley ${ }^{[52]}$ & 32 & 155 \\
\hline
\end{tabular}

*These methods were originally based on use of smooth pipe friction factors. Results presented above are from a modification that uses rough pipe friction factors. 
(a) Pre-Breakthrough

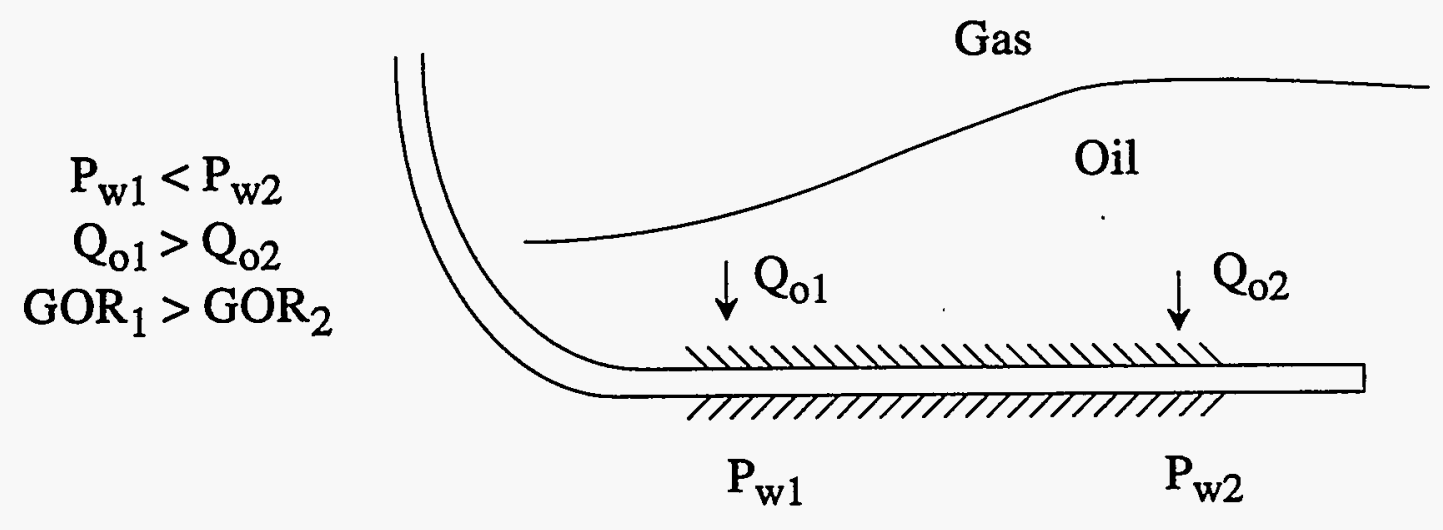

(b) Post-Breakthrough
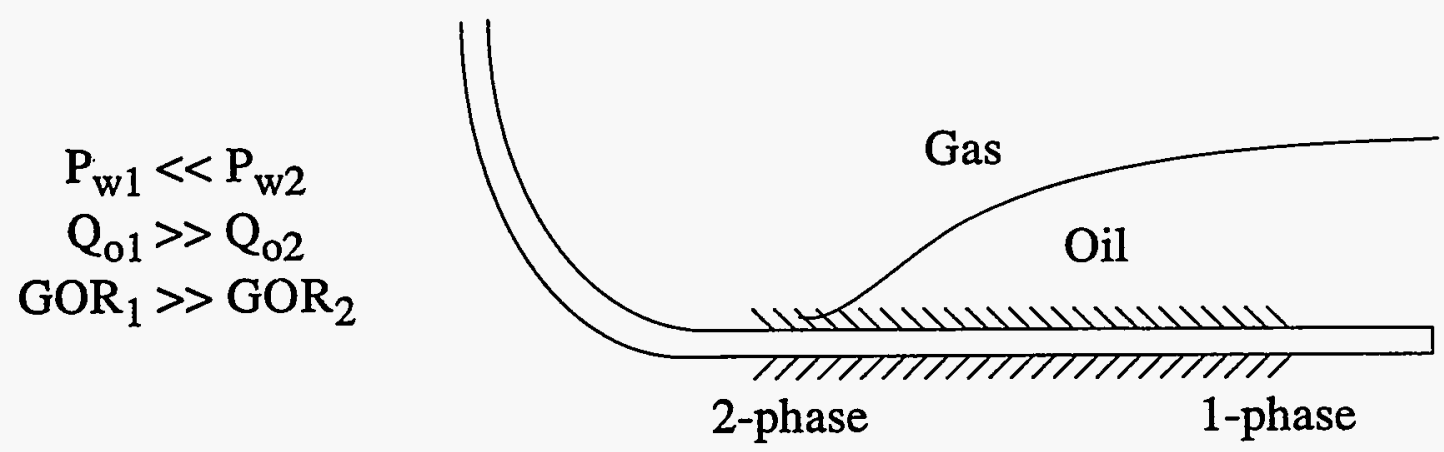

Figure 9.2: Effect of Wellbore Pressure Drop on Breakthrough of a Gas/Oil Contact 


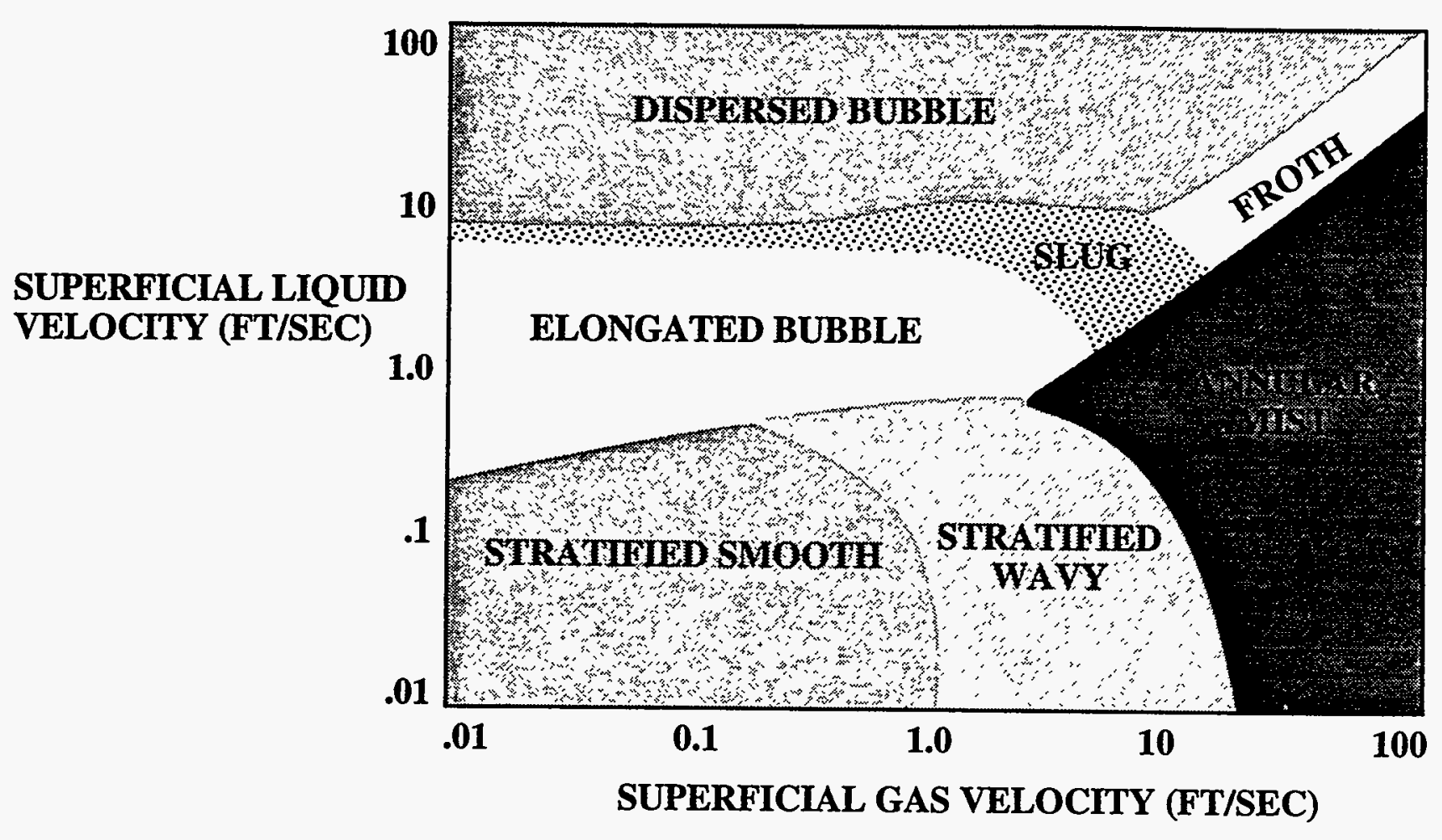

Figure 9.3: Predictions of Flow Patterns from a General Mechanistic Model for a Horizontal Pipe, Based on an Extension of the Work by Barnea [53]

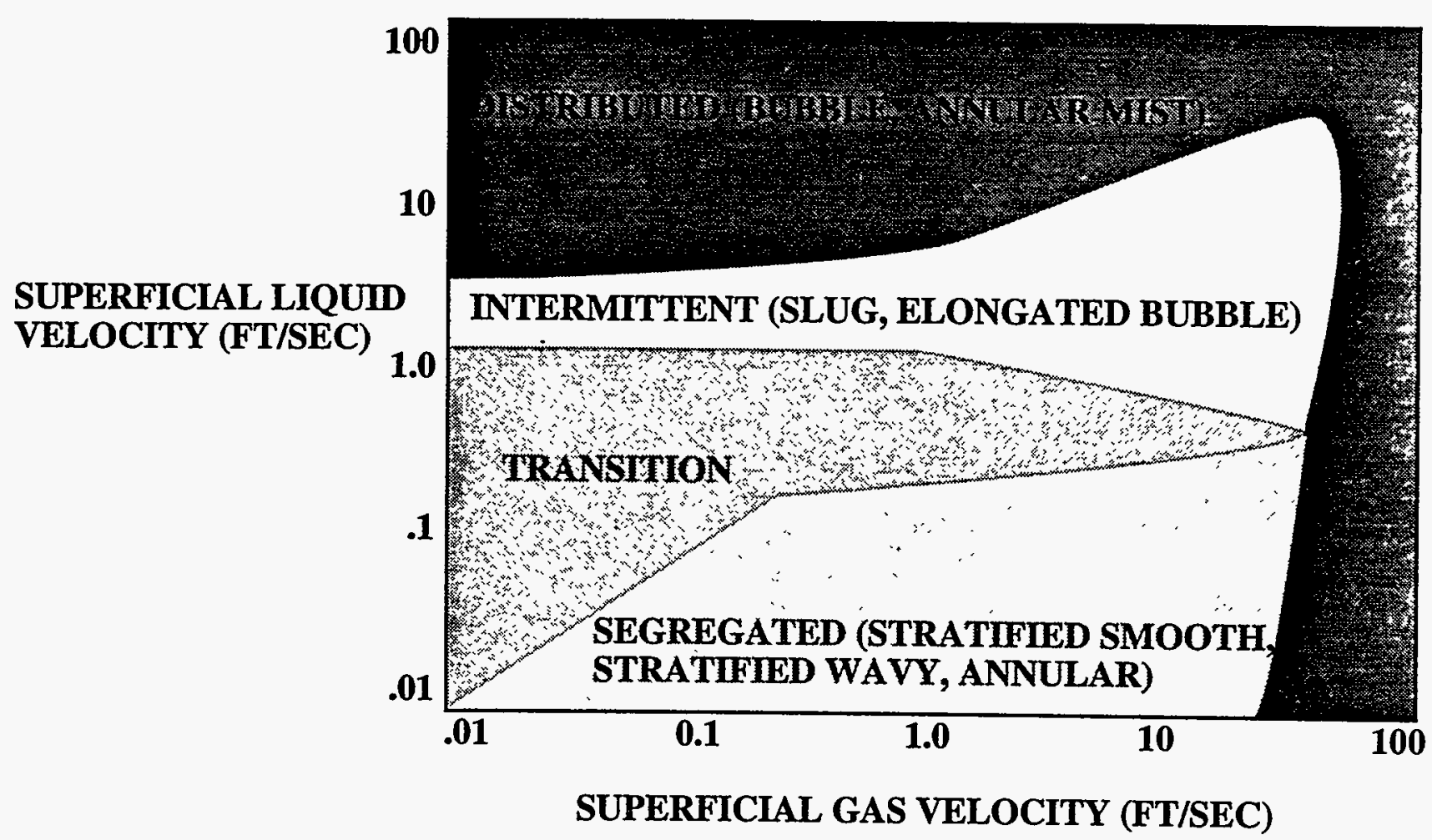

Figure 9.4: Predictions of Flow Patterns for a Horizontal Pipe from a Modification of the Beggs and Brill Method due to Brown [54] 


\section{Example Calculations for 3D-Gas Cresting Problem (Tasks 1 and 3)}

In order to test the importance of pressure drop behavior in the well and sensitivities to methods of calculation, we have set up a 3D-example reservoir study for a gas cresting problem. The chracteristics of the reservoir are summarized in Table 10.1 and shown

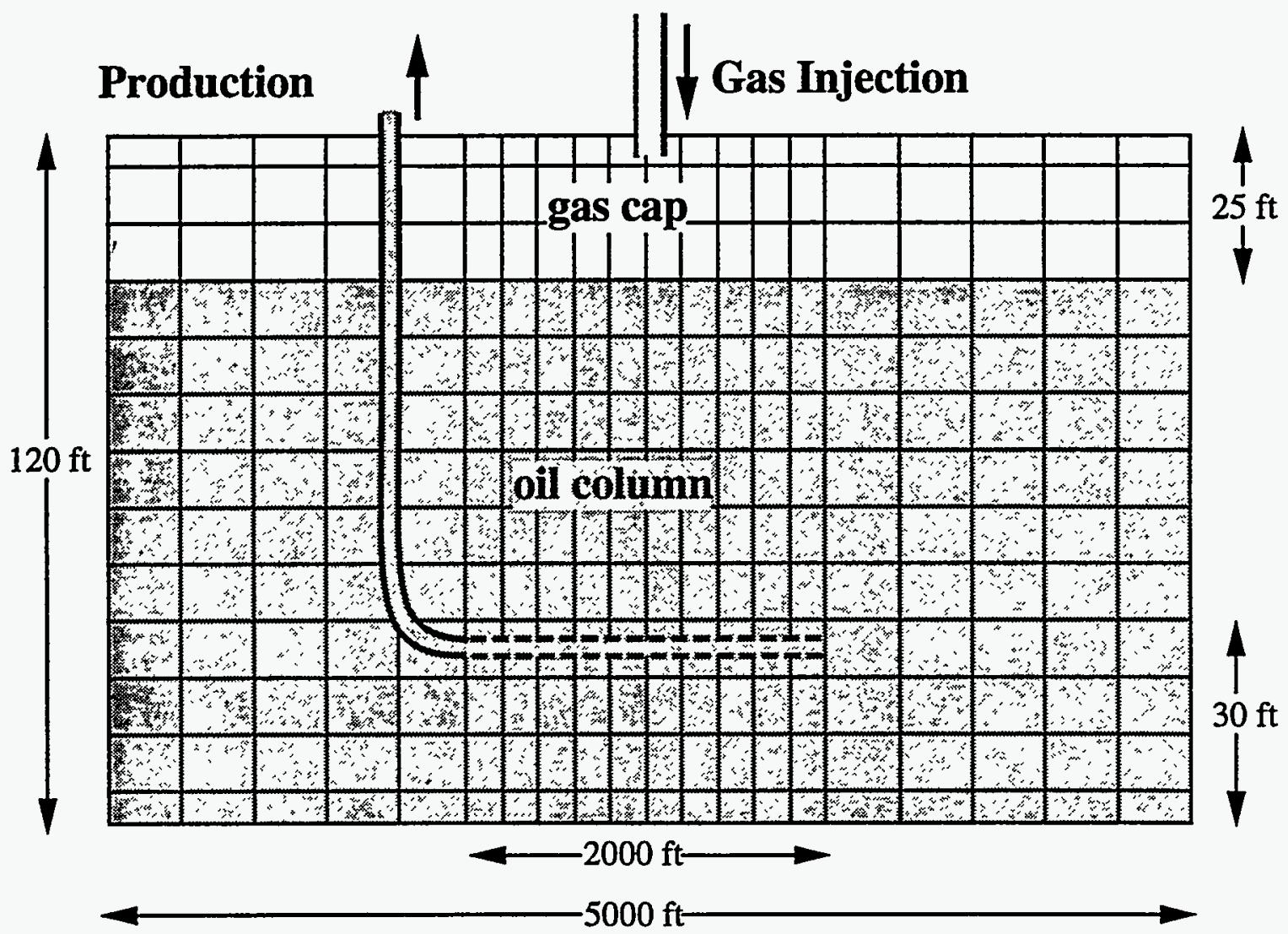

Figure 10.1: Reservoir Characteristics and Non-Uniform Gridding in the Y-Z Plane for the Homogeneous Case

schematically in Fig. 10.1. The reservoir contains an initial gas cap of thickness 25 feet, and an oil column of thickness 95 feet is produced by a horizontal well of length 2000 feet placed 65 feet below the gas/oil contact. Oil production from this well is $10,000 \mathrm{STB} /$ day and gas is injected at a fixed rate of $15 \mathrm{MMscf} /$ day into the gas cap. This keeps reservoir pressure nearly constant until gas breakthrough with declining pressure afterwards. The vertical injector is centrally placed in the gas cap, and pressure drops within the gas cap are always small. The overall length of the reservoir is $5000 \mathrm{feet}$, and its breadth is 5500 feet so that the 
Table 10.1: Summary of the Reservoir Characteristics and Parameters Used in the Cresting Simulation Example

\begin{tabular}{||ll||}
\hline Length of Reservoir & $5000 \mathrm{ft}$ \\
Width of Reservoir & $5500 \mathrm{ft}$ \\
Thickness of Reservoir & $120 \mathrm{ft}$ \\
Porosity & 0.2 \\
Horizontal Permeability & 100 and $500 \mathrm{mD}$ \\
Vertical Permeability & 10 and $50 \mathrm{mD}$ \\
\hline & \\
Initial Reservoir Pressure $\left(P_{i}\right)$ & $3472 \mathrm{psi}$ \\
Reservoir Temperature & $180^{\circ} \mathrm{F}$ \\
GOC & $95 \mathrm{ft}$ from the bottom \\
Oil Density & $58.69288 \mathrm{lbm} / \mathrm{scf}$ \\
Oil Viscosity & $2 \mathrm{cP}$ at $P_{i}$ and $180^{\circ} \mathrm{F}$ \\
Gas Density & $5.34534 \mathrm{E}-02 \mathrm{lbm} / \mathrm{scf}$ \\
Gas Viscosity & $0.0215 \mathrm{cP}$ at $P_{i}$ and $180^{\circ} \mathrm{F}$ \\
$B_{o}$ & $1.221 \mathrm{RB} / \mathrm{STB}$ at $P_{i}$ \\
$B_{g}$ & $0.813 \mathrm{RB} / \mathrm{Mscf}$ at $P_{i}$ \\
$R_{s}$ & $0.401 \mathrm{Mscf} / \mathrm{STB}$ at $P_{i}$ \\
$S_{\text {org }}$ & 0.2 \\
$S_{g c}$ & 0.02 \\
$k_{r g c w}$ & 0.8 \\
$n_{g}$ & 1.4 \\
$n_{o}$ & 2.8 \\
$k_{r g}(i)$ from equation & $k_{r g c w}\left(\frac{S_{g}(i)-S_{g c}}{1-S_{c}}\right)^{n_{g}}$ \\
$k_{\text {rog }}(i)$ from equation & $\left(\frac{1-S_{\text {org }}-S_{g}(i)}{1-S_{\text {org }}}\right)^{n_{o}}$ \\
\hline & \\
Producer Length & $2000 \mathrm{ft}$ \\
Producer Diameters & 4.28 and 6.18 in \\
Producer Areally Centered & $30 \mathrm{ft}$ above bottom \\
Production Rate of & $10,000 \mathrm{STB} /$ day \\
Relative Roughness $\epsilon_{D}$ of & 0.01942 \\
\hline & \\
Vertical Injector Diameter & $4.5 \mathrm{in}$ \\
Injector Location at & $x=2500 \mathrm{ft}, y=3000 \mathrm{ft}, z=115 \mathrm{ft}$ \\
\hline
\end{tabular}


initial oil in place is nearly 81 MMSTB. The residual oil saturation is 0.2 so that in principle about 64.8 MMSTB could be produced provided the production well was not limited by GOR constraints. A typical $k_{v} / k_{h}=0.1$ has been used and both a medium permeability $\left(k_{h}=100 \mathrm{mD}\right)$ and high permeability reservoir $\left(k_{h}=500 \mathrm{mD}\right)$ have been studied. Two internal horizontal well diameters of 6.18 and 4.28 inches have been investigated, each with a large relative roughness $\epsilon_{D}=0.0194$, which was a guess value that might be appropriate for a slotted liner completion

Two different commercial reservoir simulators have been used, namely the Intera ECLIPSE (ECL) code and the Computer Modeling Group IMEX code. The Eclipse model provides facilities for using the Peaceman Eq. (3.2) for computing $r_{o}$, and homogeneous flow properties based on the mixture volume weighted dénsity and viscosity for two-phase pressure drop in the well. IMEX uses an equivalent fracture model for the well completion factor, and the Dukler, Wicks and Cleveland [56] correlation for the two-phase flow in the well. This model therefore includes liquid hold-up. Some preliminary sensitivity calculations were made to investigate the adequacy of the grid size, and reasonable accuracies appeared to be obtained from a mesh of $20 \times 11 \times 13$ blocks. The non-uniform grid is illustrated in the $y-z$ plane in Fig. 10.1. Further refinement of the grid size near the horizontal well produced only marginal changes in results.

The adequacy of the vertical grid was also partially checked by solution for a $1 D$ problem. The grid spacing was 10 feet in the simulator results, and solution gas effects were excluded in the 1D-problem. This comparison is shown in Fig. 10.2, where the saturation profiles are very close. Gravity effects for the slow flow rate used (i.e., at the initial gas/oil contact) cause a large Buckley-Leverett cut-off saturation. This is significantly reduced in the reservoir application because of the acceleration of the gas flow in the cresting streamlines.

The histories of GOR and well pressure at the toe (BHP) for the first base case with $k_{h}=500 \mathrm{mD}$, and $D=6.18$ inches are shown in Fig. 10.3. These cases were run also assuming infinite conductivity in the well (i.e., constant internal well pressure). It is noticeable that the gas breakthrough time is earlier with ECL, but neither calculation shows much sensitivity to including pressure drop behavior in the well. There are also some variations in the ensuing GOR histories between the two codes. Examinations of the prebreakthrough saturation distributions suggests slightly different cresting behavior from the two simulators, although the $1 D$ - solutions above were very consistent. Conditions within the well are shown in Fig. 10.4 at a time when the producing GOR is $10,000 \mathrm{scf} / \mathrm{STB}$ for each calculation. Larger gas saturation values in the wellbore are predicted by the Eclipse code because it assumes no slip between the oil and gas phases. The two codes predict similar oil fluxes but differ in estimating the producing GOR along the horizontal well.

Fig. 10.5 displays the production data of a well with $D=4.28$ inches in a reservoir with $k_{h}=100 \mathrm{mD}$. A larger pressure drop at the toe is predicted by the two codes compared - to the base case. The producing GOR values however again do not show much sensitivity to the consideration of two-phase pressure drop in the well. Conditions along the well are shown in Fig. 10.6 where, relative to the base case, a much larger pressure drop is predicted by both codes. It should be pointed out that because the ECL and IMEX codes give close estimates for BHP at a GOR of 10,000 scf/STB (see Fig. 10.5), the pressures along the length of the well (>1000 ft) are also nearly the same. The remaining parameters along the well in Fig. 10.4 show similar behavior to the base case. 


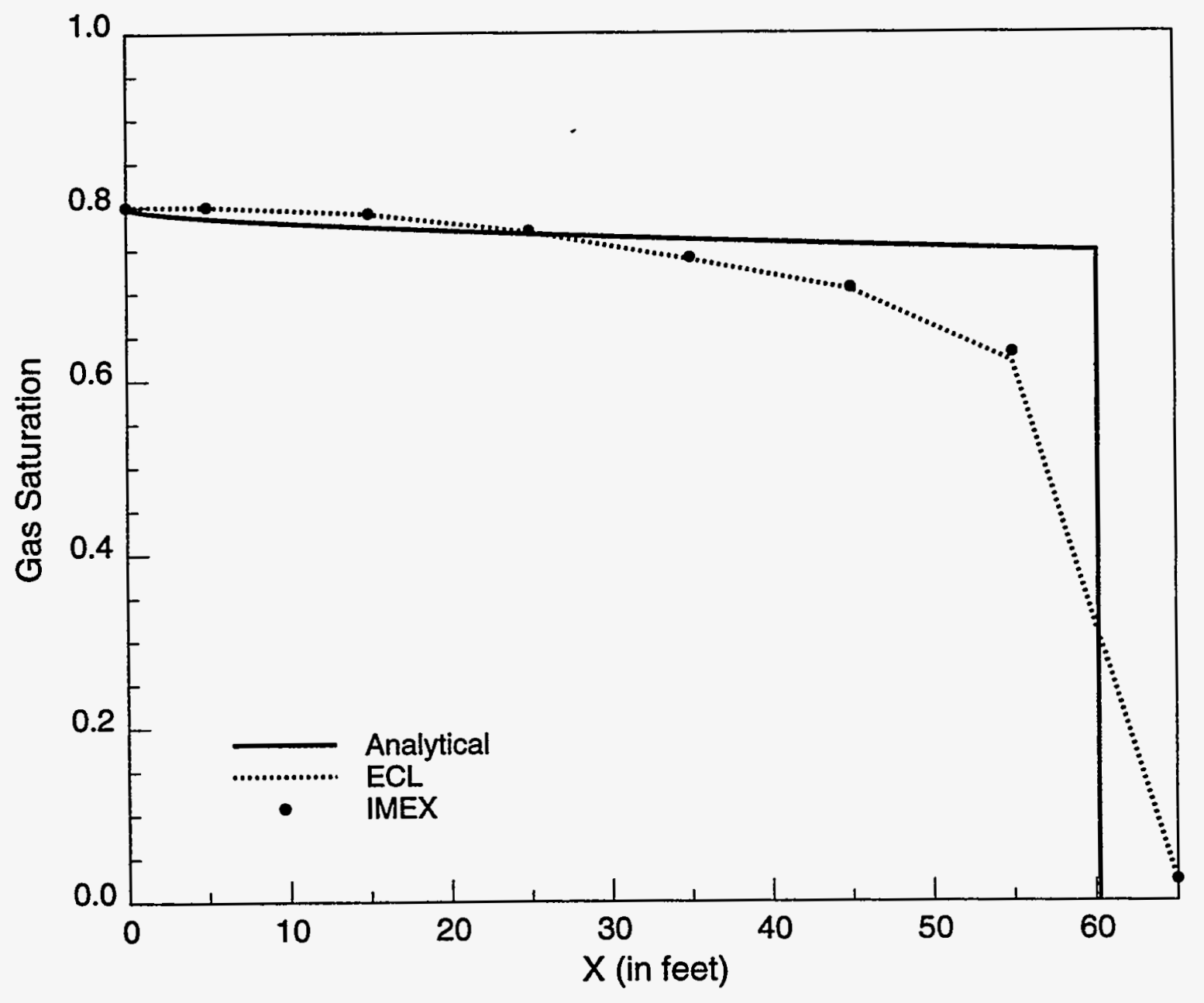

Figure 10.2: Gas Saturation Profiles for the 1D-Problem 


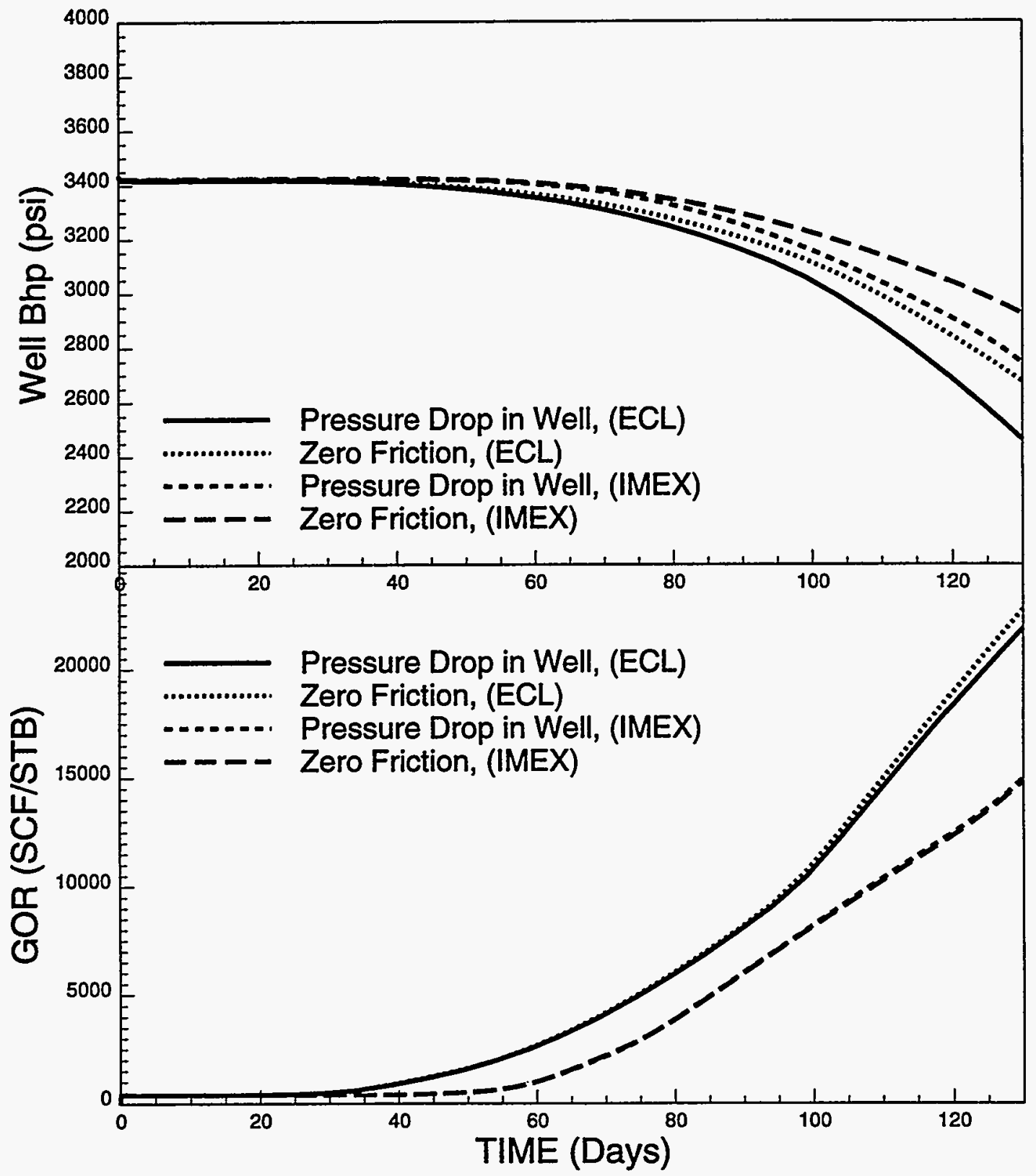

Figure 10.3: Production Data of Well (Dia=6.18in) with Reservoir Permeability of $500 \mathrm{mD}$ 


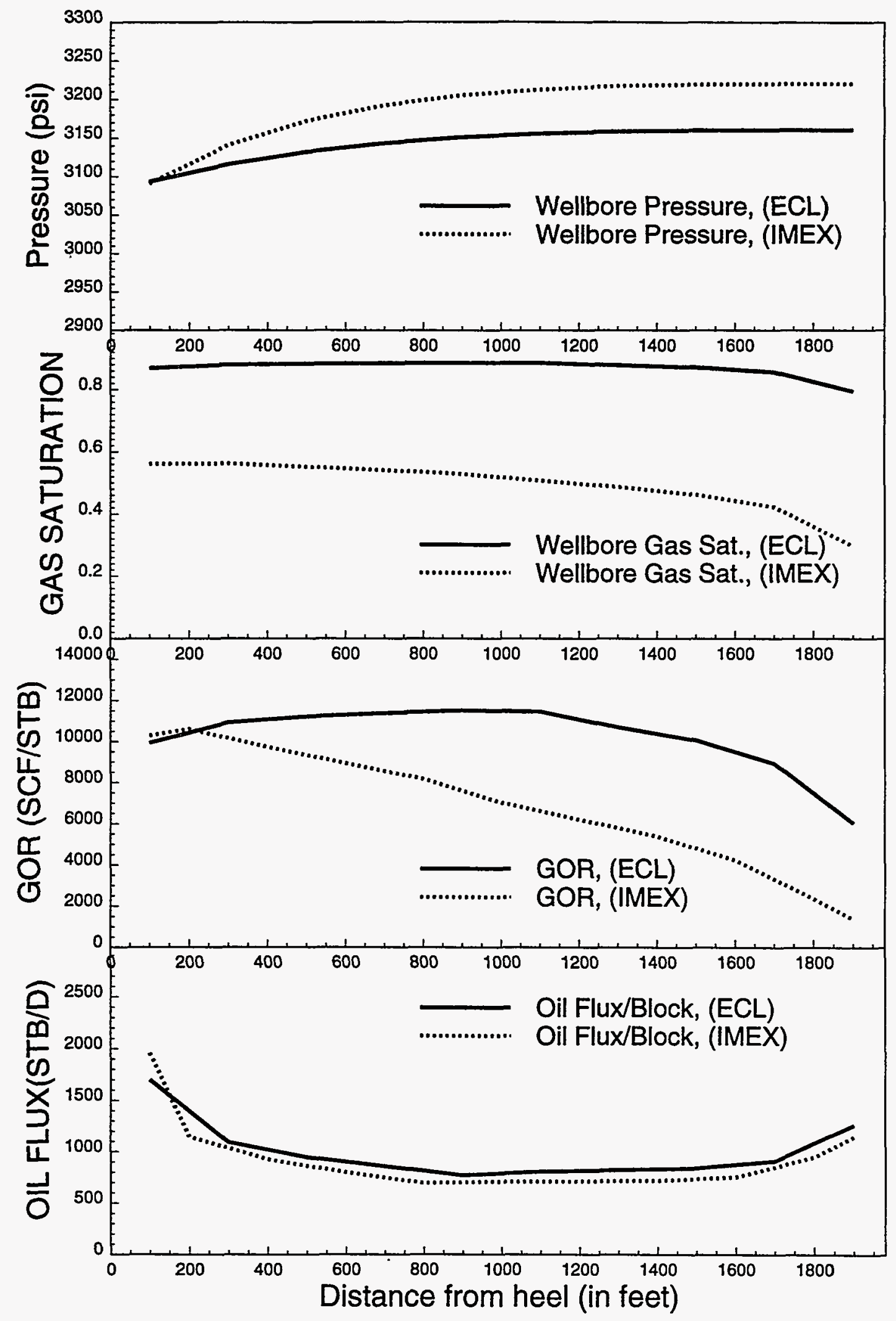

Figure 10.4: Parameters Along Horizontal Well (Dia=6.18 in) for Reservoir Permeability of $500 \mathrm{mD}$ 


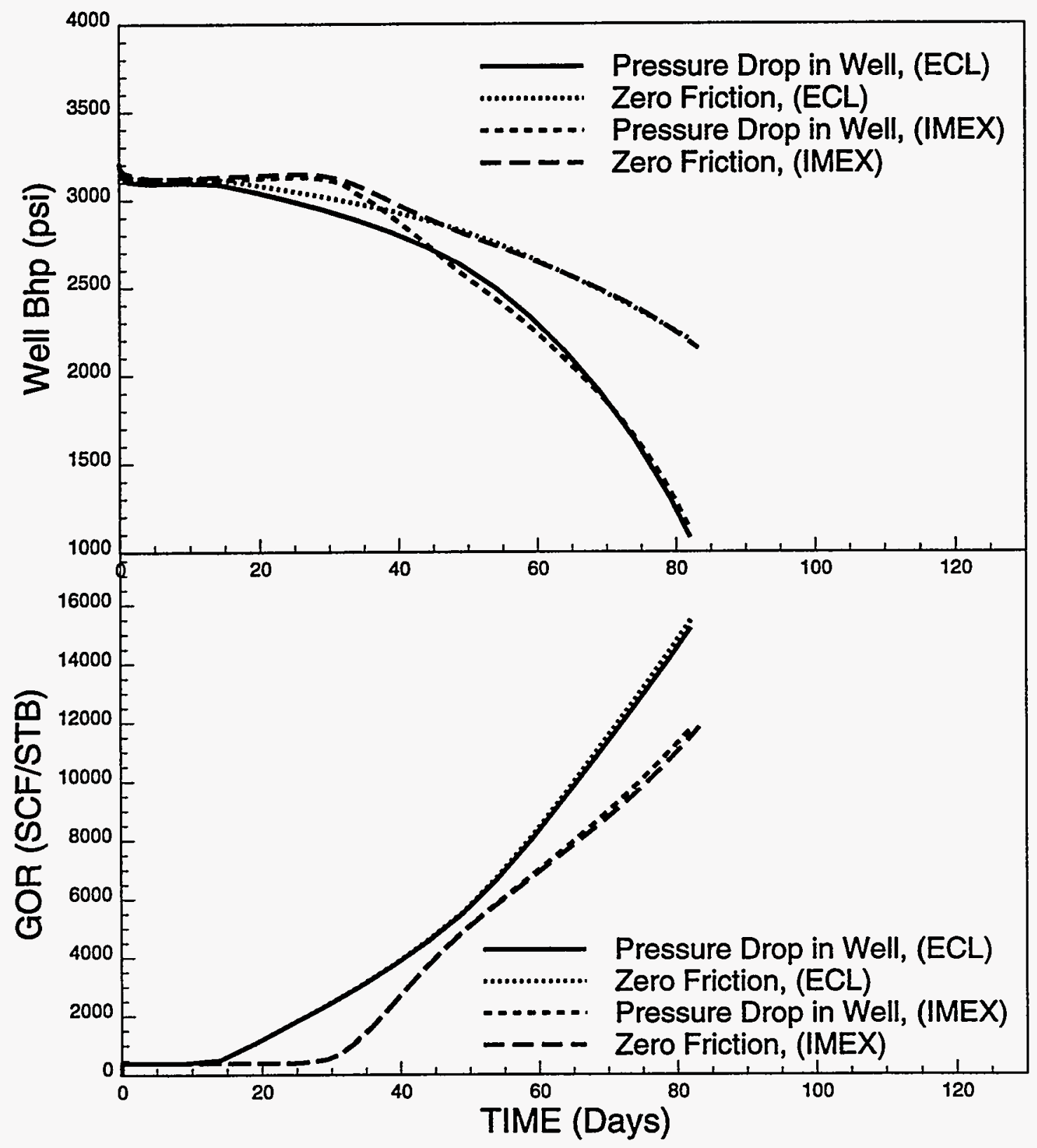

Figure 10.5: Production Data of Well (Dia=4.28in) with Reservoir Permeability of $100 \mathrm{mD}$ 


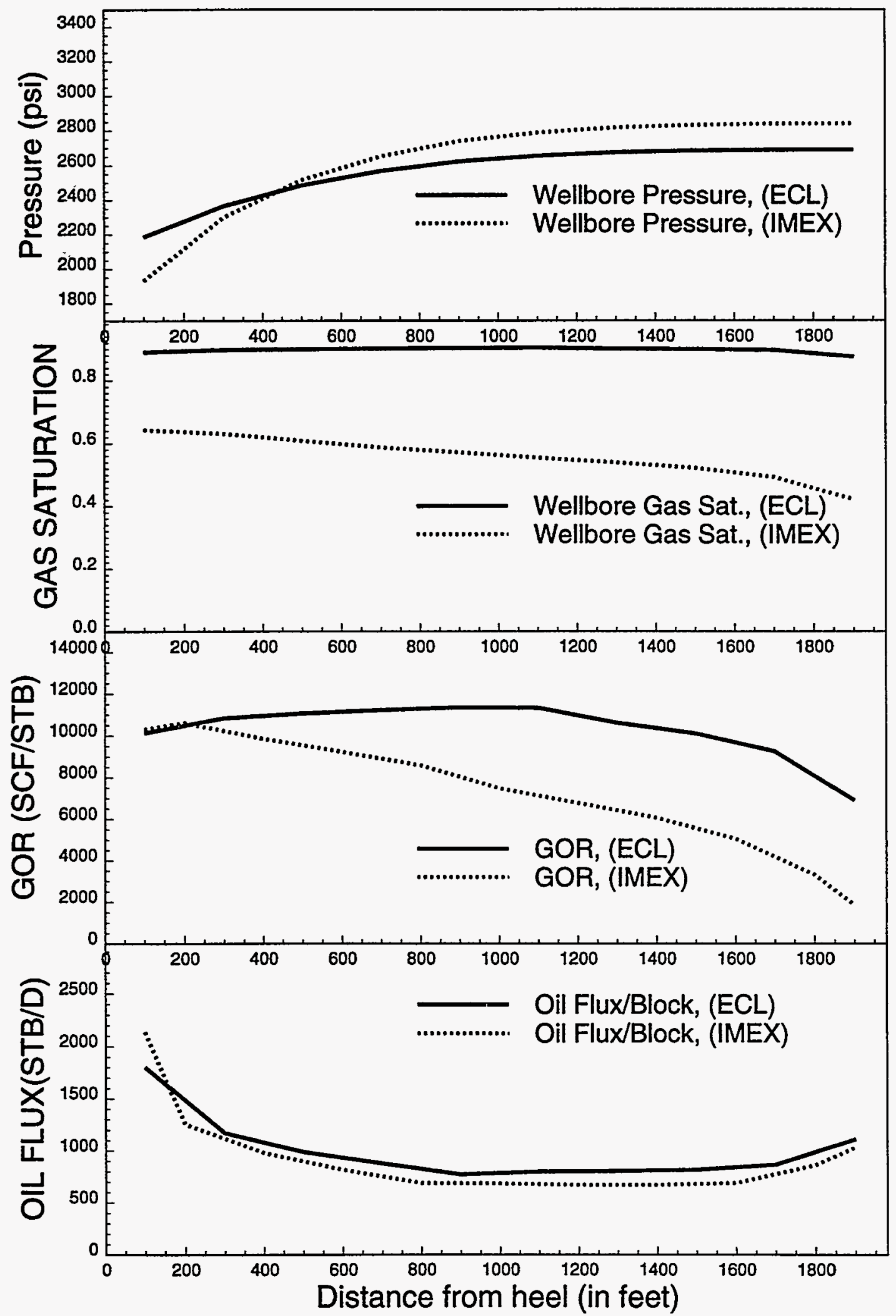

Figure 10.6: Parameters Along Horizontal Well $(\mathrm{Dia}=4.28$ in) for Reservoir Permeability of $100 \mathrm{mD}$ 
The results of the previous case $\left(k_{h}=100 \mathrm{mD}\right)$ with a larger well diameter, $D=6.18$ inches, are shown in Figs. 10.7 and 10.8. The drop in BHP is less because of the larger well diameter but the breakthrough time and the producing GOR are almost unchanged as shown in Figs. 10.5 and 10.7. The conditions along the well in Fig. 10.8 also exhibit similar trends to Fig. 10.6.

All the above examples unexpectedly showed that gas breakthrough time and the subsequent build-up of the GOR is not dependent on including the wellbore pressure drop calculation. Although the two codes give different GOR histories from each other, there is consistency between them on the apparent insensitivity to well pressure drop calculation. The independence of the GOR-history from wellbore pressure drop is akin to the constancy in single phase productivity associated with neglecting the well friction which was found to occur for larger diameter wells by Novy [58]. However, wellbore friction does become important at higher flow rates and with larger roughness or reduced pipe diameter [51]. We believe a number of factors contribute to the invariance in GOR behavior as enumerated below.

(i) The strongest factor influencing gas breakthrough time is the well production rate, which has been controlled to be equal in all the models, (i.e. 10,000 STB/day). The more usual field application would be to operate the well at its maximum deliverability, which will then vary between the cases.

(ii) The reservoir is uniform, and for both $k_{h}=500 \mathrm{mD}$ and $100 \mathrm{mD}$, it has a sufficiently large permeability to allow substantial horizontal flow adjacent to the wellbore. This causes the reservoir pressures near the well to be closely coupled to the almost linear variation within the well. Thus $P_{o}-P_{w}$ is almost constant and the oil in-flux along the well is nearly uniform (see Fig. 10.6, for example). Most of any residual variation in in-flow rate is offset by horizontal flow in the reservoir, which allows $\partial P / \partial z$ near the gas/oil contact to remain almost uniform. Thus the resulting development of gas saturation within the crest does not vary by much for a given height above the well, and the breakthrough time is insensitive to wellbore pressure drop.

Non-linear variation in pressure within the well and the associated in-flow rates can be driven by reservoir non-uniformities. To test this hypothesis a slightly heterogeneous reservoir description was examined with the left half of the problem having $k_{h}=100 \mathrm{mD}$ and the right half $k_{h}=500 \mathrm{mD} . k_{v} / k_{h}=0.1$ was retained everywhere. The resulting GOR histories, shown in Fig. 10.10 now shift significantly with inclusion of the wellbore pressure drop in the Eclipse calculation, although less so with IMEX. The effect of wellbore pressure drop at the heel of the well is to increase the advance of the retarded gas/oil contact in the $100 \mathrm{mD}$ permeability zone, thereby making this contact more uniform than in the case of neglecting wellbore pressure drop. This then gives a more favorable GOR history. However, note that the $\mathrm{BHP}$ also declines more rapidly with the pressure drop included. The resulting gas saturations and oil fluxes along the well also show stronger variations, as indicated in Fig. 10.11 , particularly at the interface between the permeability changes. When the sequence of the permeability variations was reversed, so that the $500 \mathrm{mD}$ region is close to the heel of the well, the unexpected result was found that the GOR history was again rather insensitive to wellbore pressure drop. This is explained by the fact that now most of the flow enters 


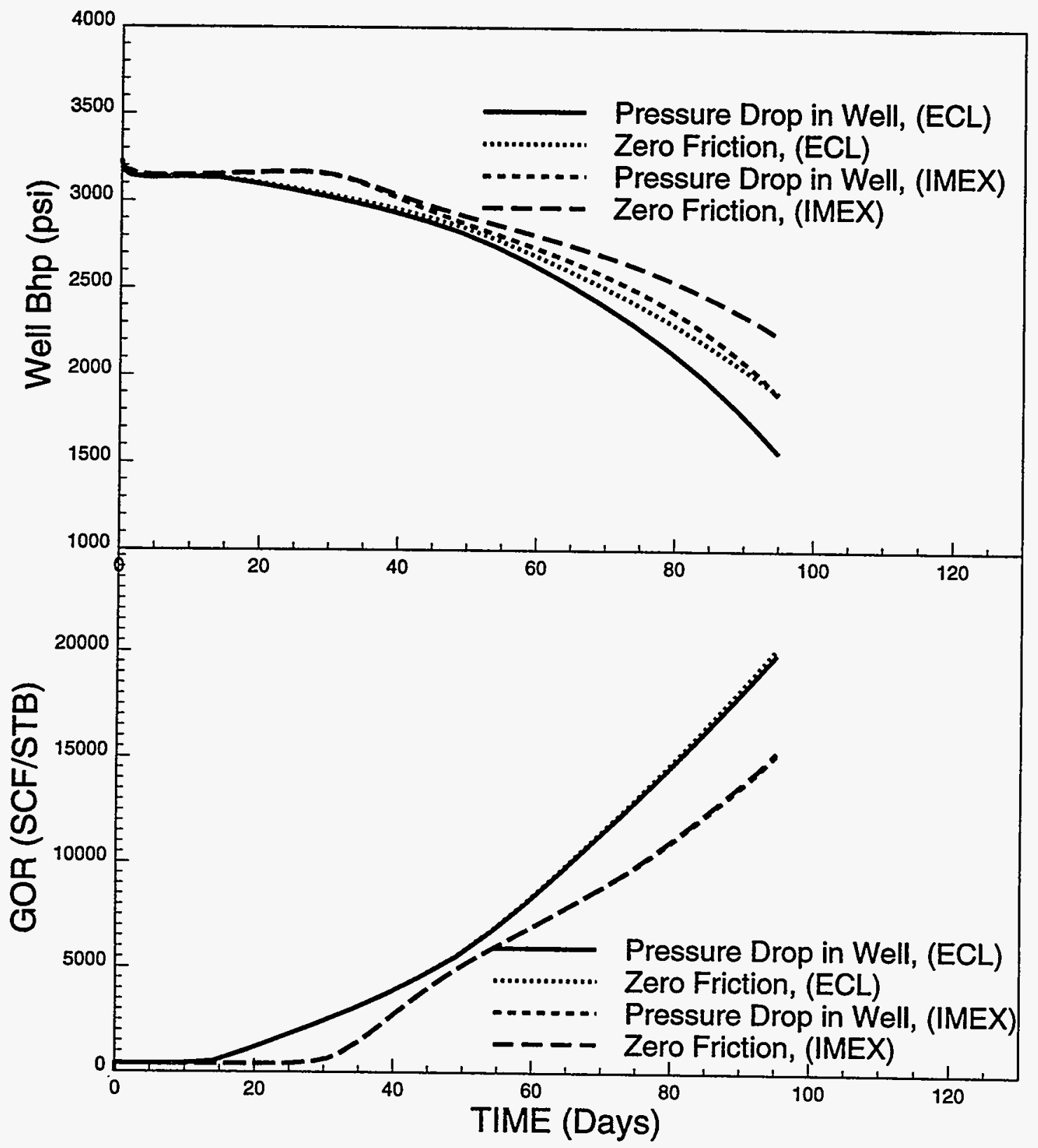

Figure 10.7: Production Data of Well (Dia $=6.18 \mathrm{in}$ ) with Reservoir Permeability of $100 \mathrm{mD}$ 


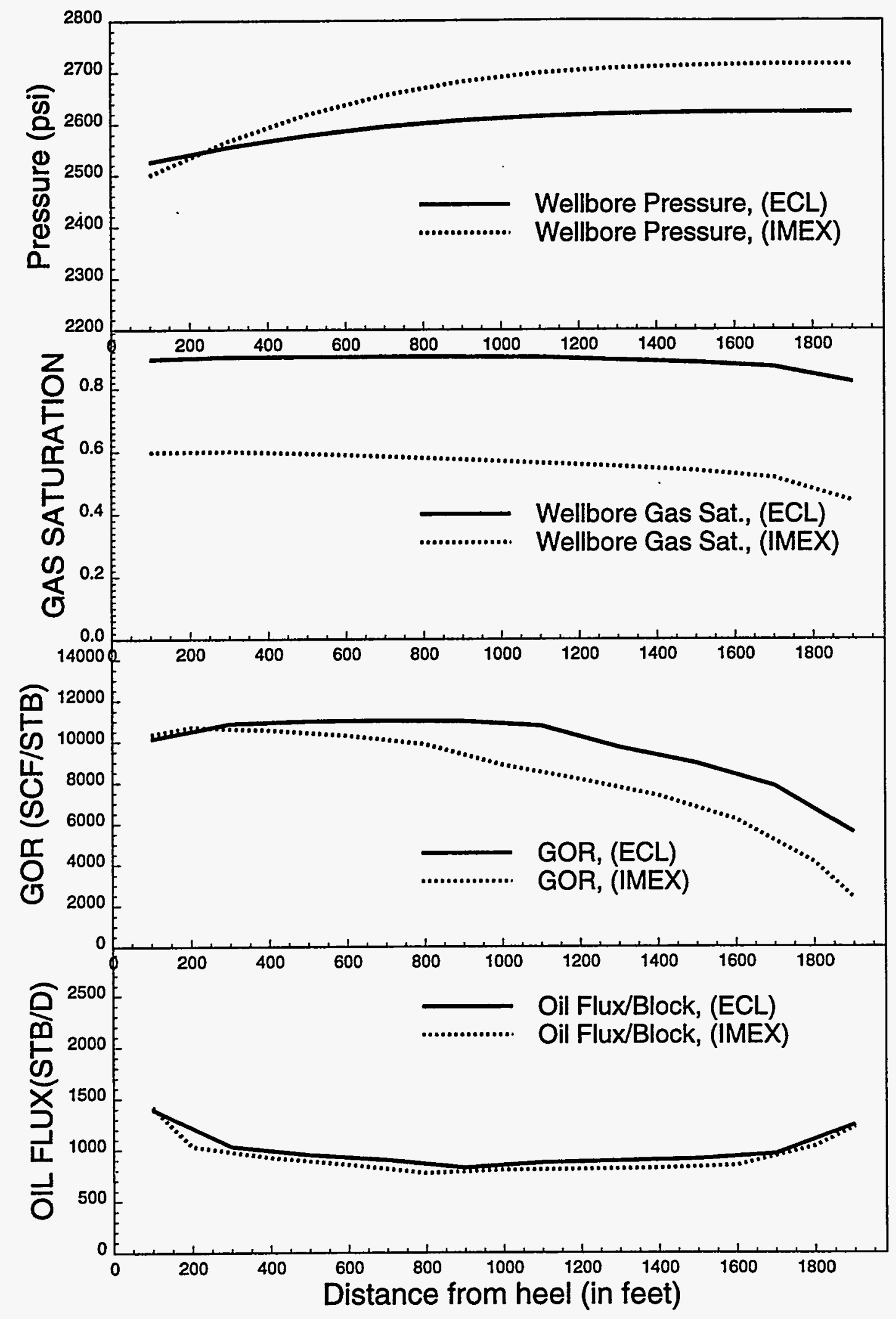

Figure 10.8: Parameters Along Horizontal Well (Dia=6.18 in) for Reservoir Permeability of $100 \mathrm{mD}$ 
through the downstream half of the well, rather than the upstream half, so that the well total pressure drop is reduced. Furthermore, because the increased drawdown now occurs adjacent to the reservoir section with high permeability, the reservoir can provide a large component of horizontal flow, so that the gas/oil interface descends in a relatively uniform manner.

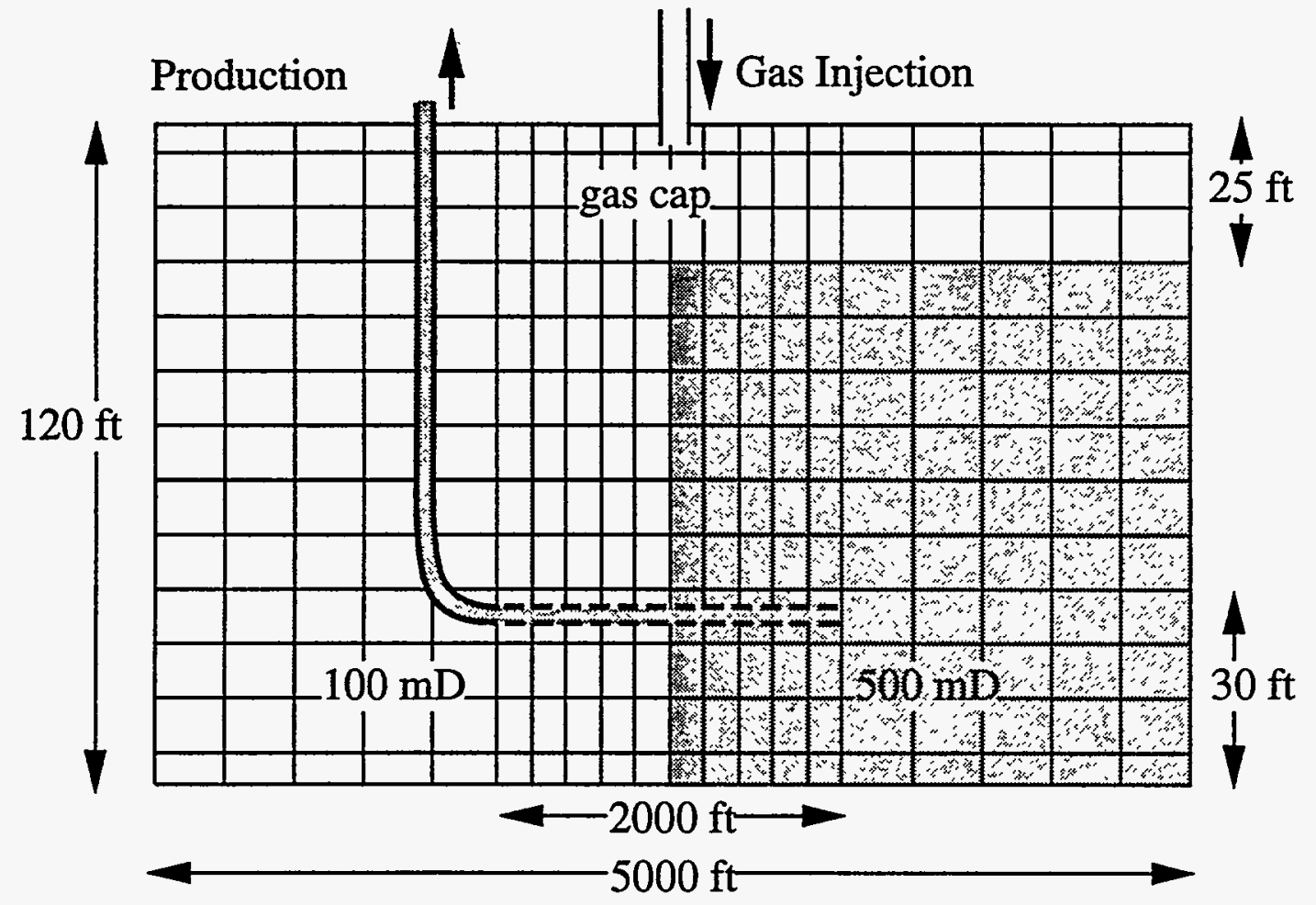

Figure 10.9: Reservoir Characteristics and Non-Uniform Gridding in the Y-Z Plane for the Non-Homogeneous Case

To test the influence of constant production rate identified in item (i) above, a case was set up, with well controls perhaps more appropriate to a field operating condition, in which a fixed injection rate of gas into the gas cap was imposed at $12.3 \mathrm{MMscf} /$ day, but now a fixed limit on bottom hole pressure (BHP $=2,920 \mathrm{psi}$ ) was assumed to control the production well. For the uniform reservoir with $k_{h}=100 \mathrm{mD}$, the production well commences producing at about $18,000 \mathrm{STB} /$ day, but subsequently declines in rate to about $5000 \mathrm{STB} /$ day, as illustrated in Fig. 10.12. The resulting GOR history now depends on inclusion of wellbore pressure drop, with the GOR being reduced when this is included. However, the associated oil flow rate is also slightly reduced. Comparison of conditions along the well for this case, shown in Fig. 10.13, with the corresponding constant flow rate case given in Fig. 10.4, does not suggest any significant increase in non-uniformity.

When the effects of the adverse permeability variation and more realistic well controls were applied simultaneously, the variations of the GOR history between infinitely conducting and resistive wells became more obviously divergent. In this case the production rate was limited to $20,000 \mathrm{STB} /$ day until the BHP limit of 2920 psi was attained; the $500 \mathrm{mD}$ section of the well otherwise gives very high initial flow rates. The production profiles are given 


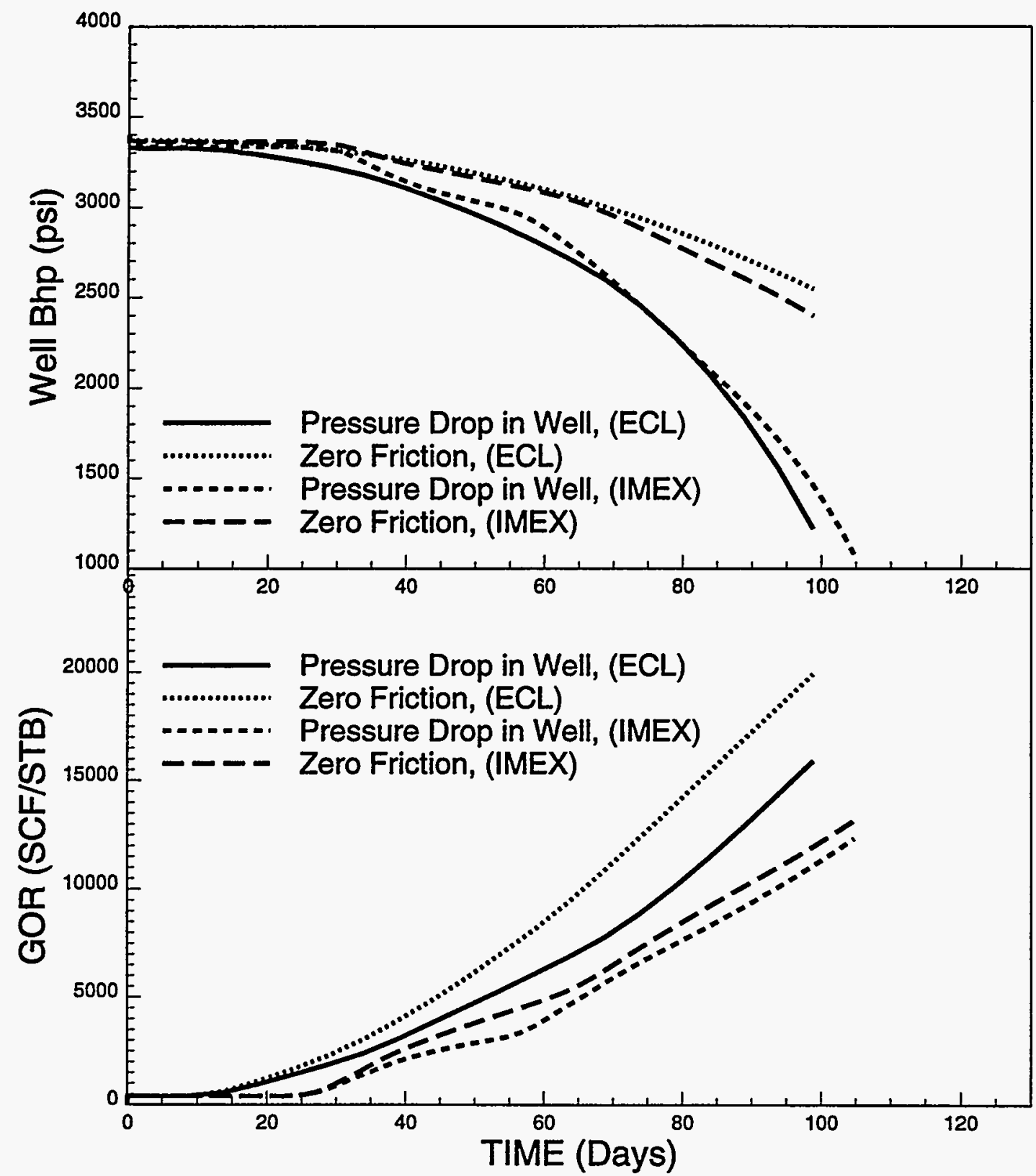

Figure 10.10: Production Data of Well (Dia=4.28in) with Reservoir Permeability of 100-500 $\mathrm{mD}$ 


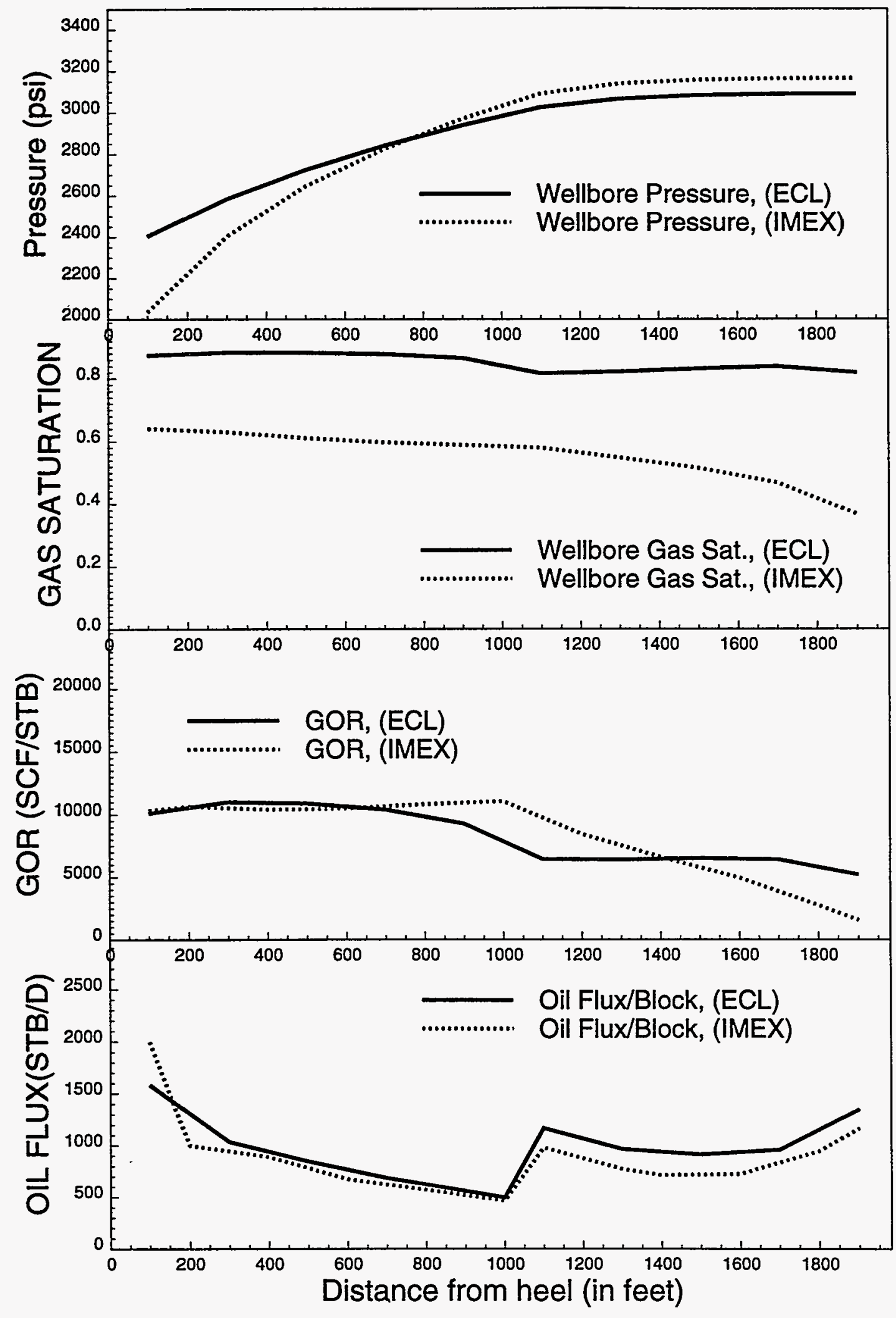

Figure 10.11: Parameters Along Horizontal Well $(\mathrm{Dia}=4.28$ in) for Reservoir Permeability of $100-500 \mathrm{mD}$ 


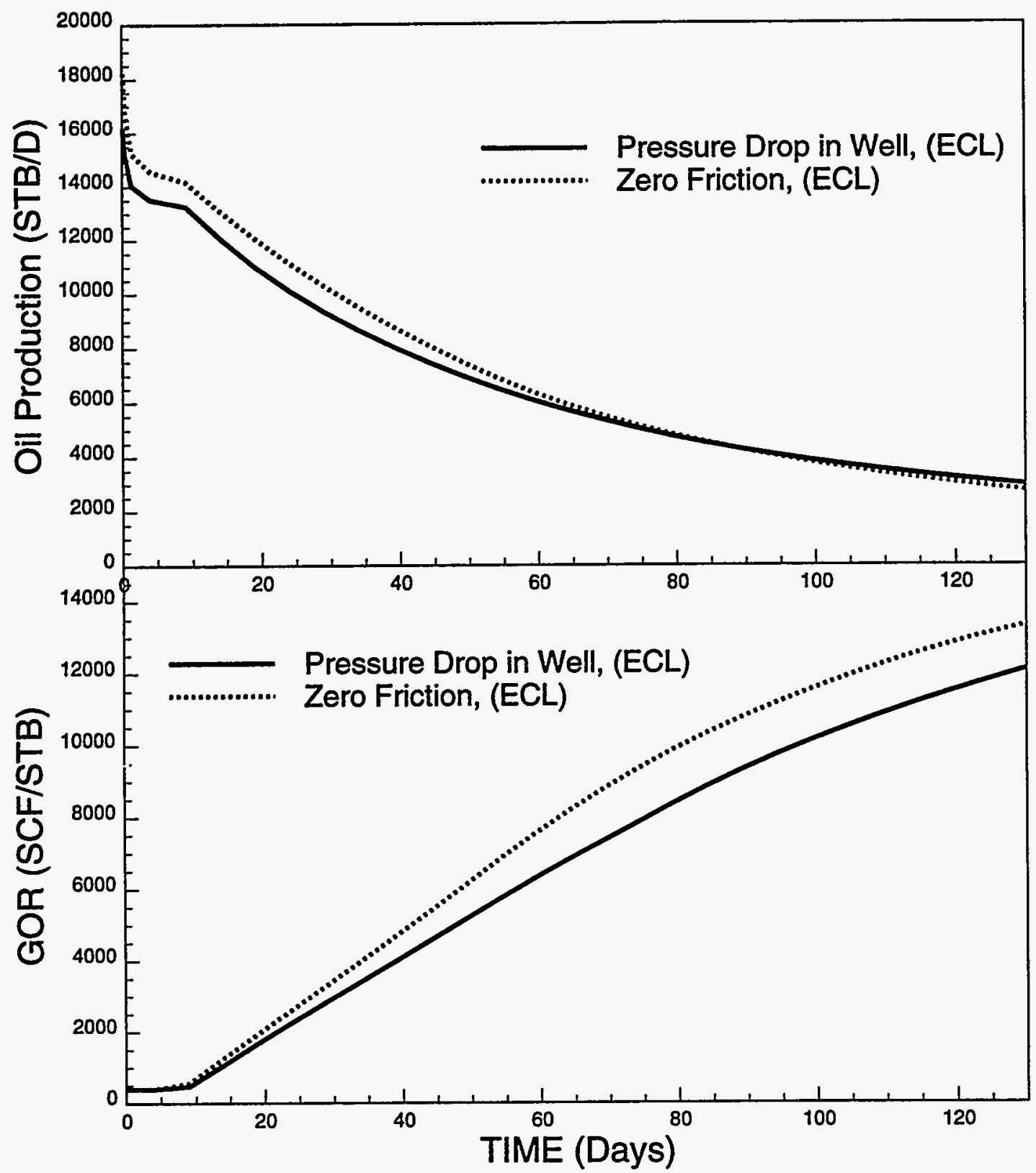

Figure 10.12: Production Data of Well (Dia=4.28in) for Fixed BHP with Reservoir Permeability of $100 \mathrm{mD}$ 


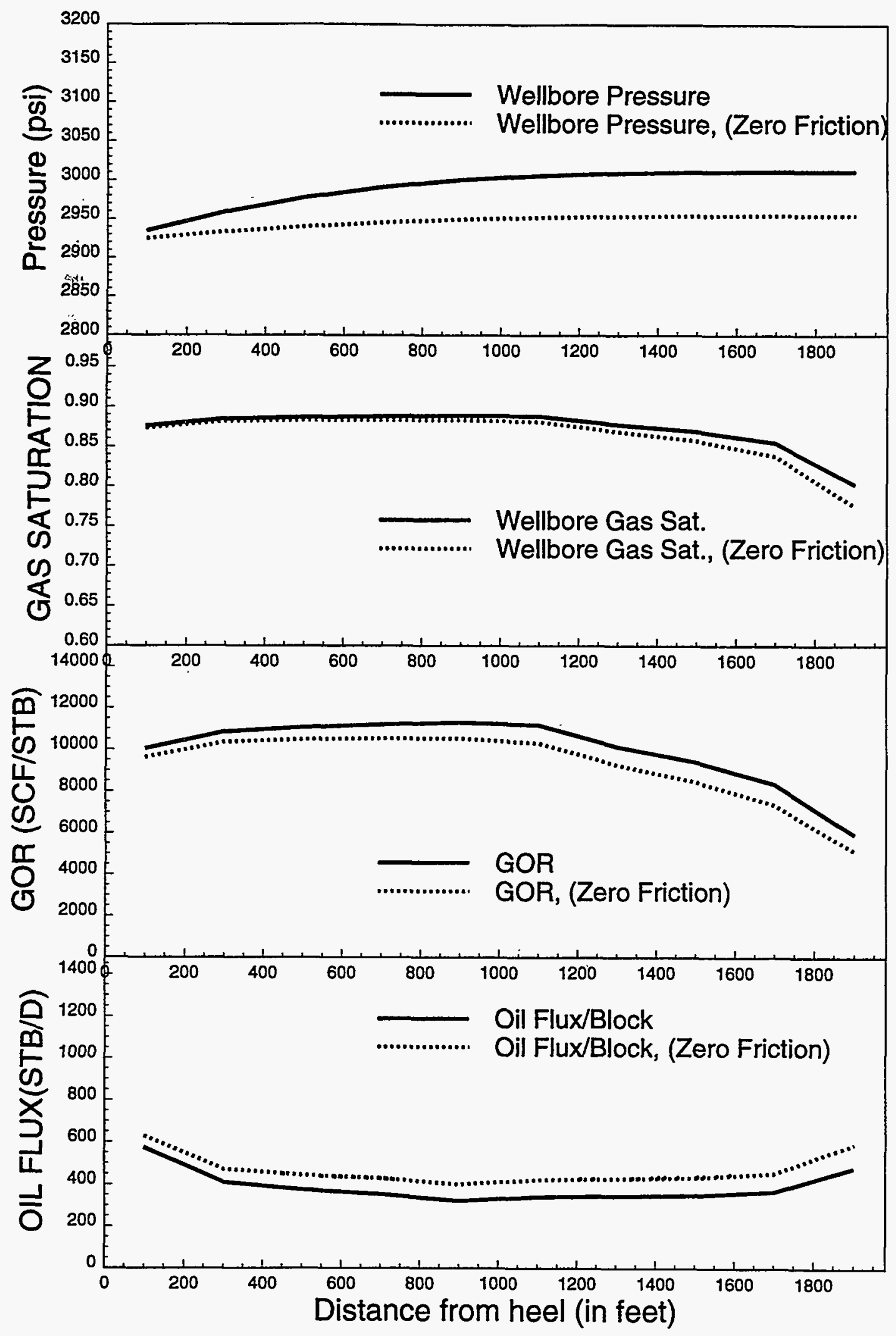

Figure 10.13: Parameters Along Horizontal Well (Dia=4.28 in) for Fixed BHP with Reservoir Permeability of $100 \mathrm{mD}$ 
in Fig. 36, where for example an operating limit on GOR of say 10,000 scf/STB would be reached in 36 days without pressure drop in the well, but this is extended to 112 days when the well resistance is included. The corresponding total oil productions up to this cut-off are $6.25 \times 10^{5} \mathrm{STB}$ and $9.60 \times 10^{5} \mathrm{STB}$ respectively. The GOR history for the infinitely conducting well is no longer monotonic. This occurs because the pressure fall-off near the well allows solution gas to be evolved which is preferentially produced before the gas cap has advanced enough to dominate in a final period of rising GOR (not shown in the figure).

Some further sensitivity to well pressure drop calculation is shown in Fig. 10.14 for a case when a skin factor of $S=10$ was also assigned to the lower permeability half of the well, with the second half having $S=0$, assuming damage only occurs to the low permeability rock near the heel of the well. A uniform skin assumption of $S=5$ along the entire length produced very little additional effect. 


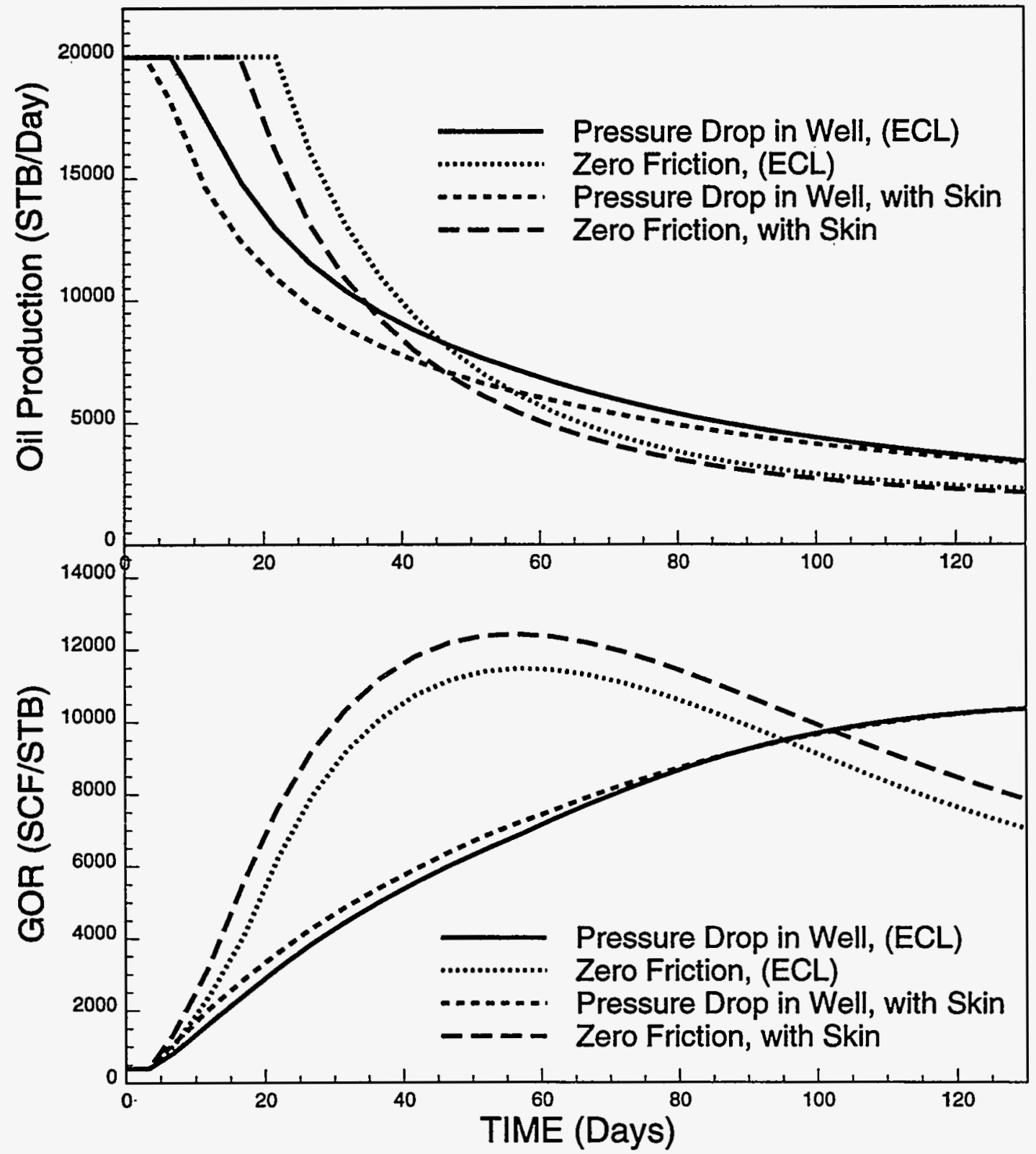

Figure 10.14: Production Data of Well ( $\mathrm{Dia}=4.28$ in) for Fixed BHP with Reservoir Permeability of $100-500 \mathrm{mD}$ 


\section{Development of a Mechanistic Model for Multiphase Flow in Horizontal Wells (Tasks 1 and 3)}

This chapter is an overview of the work of Prof. Khalid Aziz and Nick Petalas, a professional programmer in the department, on the development of a general mechanistic model.

\subsection{Introduction}

Typical horizontal wells consist of long sections that are nearly horizontal and vertical or deviated sections to bring the fluids to the surface. Accurate pressure drop calculations in such wells are required for optimum well design. A number of models are available in the literature to model multiphase flow in pipes. Some of these were developed using horizontal pipe inclinations while others are based on vertical upflow. Although models that can be used at all pipe inclinations do exist, their ability to properly account for variations in fluid properties and pipe diameter is generally limited. Furthermore, large discontinuities can be encountered across flow pattern transition boundaries which cause corresponding discontinuities in the pressure drop and holdup correlations.

Since typical horizontal wells involve variations in pipe diameter and can include inclinations at all angles (including downflow), a model is being developed that can be used under these conditions. Although some empirical correlations are, by necessity, used, the model is largely based on mechanistic considerations and thus provides the required dependence on fluid properties and pipe geometry. Where empirical correlations are dictated, many of those available in the literature are compared and the ones resulting in the smoothest transitions across flow pattern boundaries are used.

Graphical representation is paramount in ensuring that realistic results are obtained and many two-dimensional as well as three-dimensional plots are employed to observe the behavior of key parameters over a large range of flow rates and for different fluid properties. Some of these parameters include the frictional and hydrostatic pressure drop components, liquid holdup, liquid height during stratified flow and liquid film thickness during annular flow.

\subsection{Model Overview}

By assuming that a particular flow regime is present, and solving the momentum balance equations for certain quantities that determine its characteristics, the stability of the flow pattern is examined. If the flow pattern is shown to be stable, the procedure is terminated, the pressure drop and holdup being obtained directly from the momentum balance equations. If the flow pattern cannot exist under the specified conditions, a new flow pattern is assumed and the procedure is repeated until a stable flow pattern is determined.

The flow patterns which are considered are: 
- Stratified Flow (smooth and wavy)

- Annular-Mist Flow

- Intermittent Flow (Slug, Elongated Bubble)

- Dispersed Bubble

- Bubble Flow

- Froth Flow

The procedure begins by assuming stratified flow and calculating the liquid height. If the flow pattern is stable, it is then determined whether the flow is smooth or wavy. The pressure drop and holdup are obtained from the calculated liquid height. An empirical correlation is used to determine the gas-liquid interfacial friction factor.

If stratified flow is not possible, it is assumed that the flow is annular-mist and the thickness of the liquid film is calculated by writing the momentum balance over the gas core and the liquid film. This is then used to establish whether annular-mist flow is possible, and if so, to calculate the pressure drop and holdup. The calculation of the liquid film thickness requires that empirical correlations be used for the determination of the gas-liquid interfacial friction factor and for the fraction of liquid entrained in the gas core.

Once stratified flow and annular-mist flow are eliminated, the flow pattern can be either intermittent, bubble, dispersed bubble, or froth which is treated as a transition between annular-mist and intermittent and between annular-mist and dispersed. The intermittent flow region stability is based on the holdup in the liquid slug which is evaluated from an empirical correlation. The elongated bubble region is defined as the region within intermittent flow where there is no gas entrained in the liquid slug. The remainder of the intermittent flow region is identified as slug flow. The pressure drop and holdup for intermittent flow are determined from momentum balance, although empirical correlations are required to calculate the translational velocity of the slug, the velocity of the dispersed bubbles and the liquid holdup in the slug as previously mentioned.

Bubble flow can exist if the pipe is large enough and the inclination steep enough to prevent bubbles from rising to the top of the pipe. This can be established by determining the bubble rise velocity which is also used to calculate holdup. The pressure drop is calculated by assuming a homogeneous mixture density and a friction factor based on liquid density and viscosity and the mixture velocity.

Dispersed bubble flow is assumed to exist when the liquid rate is high enough such that the gas entrained in the liquid slug reaches or exceeds the maximum volumetric packing density causing the slug to break up. The gas rate must also be such that the same packing density is not exceeded for the total flowing fraction. Holdup is calculated by assuming no slip between phases and pressure drop is based on the homogeneous mixture properties.

If none of the above flow patterns are shown to exist, froth flow is assumed and the holdup and pressure drop are calculated by linear interpolation across the appropriate boundaries. 


\subsection{Results}

Preliminary results show generally smooth transitions across flow pattern boundaries for most pipe inclinations. The more severe discontinuities are encountered during downflow when using air and water as the two phases. The fact that the air/water system produces the most severe discontinuities is to be expected given the large differences between the densities and viscosities of the two phases. Further refinement of the stratified wavy to annular-mist transition mechanism is expected to overcome these problems.

Figure 11.1 shows the flow map generated by the mechanistic model for the case of an oil/gas system at flowing conditions. The properties are given in Table 11.1 below:

Table 11.1: Fluid Properties Used in the Mechanistic Model

\begin{tabular}{||ll||}
\hline Pipe Diameter & $6.18 \mathrm{in}$ \\
Interfacial Tension & $20.0 \mathrm{dyne} / \mathrm{cm}$ \\
Pressure & $2,340.4 \mathrm{psi}$ \\
Absolute Roughness & $0.01 \mathrm{ft}$ \\
Gas Density & $8.139 \mathrm{lbm} / \mathrm{ft}^{3}$ \\
Liquid Density & $52.53 \mathrm{lbm} / \mathrm{ft}^{3}$ \\
Gas Viscosity & $0.018 \mathrm{cP}$ \\
Liquid Viscosity & $2.757 \mathrm{cP}$ \\
\hline
\end{tabular}

The frictional pressure drop (per unit length) surface plot corresponding to this flow pattern map is shown in Fig. 11.2 where the flow patterns are also indicated. It can be seen that small discontinuities exist at the stratified/intermittent and the stratified/annular transitions. The in-situ liquid fraction (liquid holdup) surface plot is shown in Fig. 11.3 where the same type of discontinuities can be seen.

When the pipe is inclined, even for small angles, the flow patterns can change significantly. This is shown in Fig. 11.4 for a downward angle of $5^{\circ}$ and in Fig. 11.5 for an upward angle of $5^{\circ}$. At even higher angles, bubble flow can exist as shown in Fig. 11.6 for an upward pipe inclination of $80^{\circ}$.

\subsection{Future Development}

The stratified to annular-mist transition requires some further refinement in order to reduce the size of discontinuities encountered in downflow.

A database is being created wherein extensive data will be collected for the purposes of verifying the results produced by the mechanistic model. This will allow for final tuning of the model and help substantiate the selection of empirical correlations that are used within the model. 


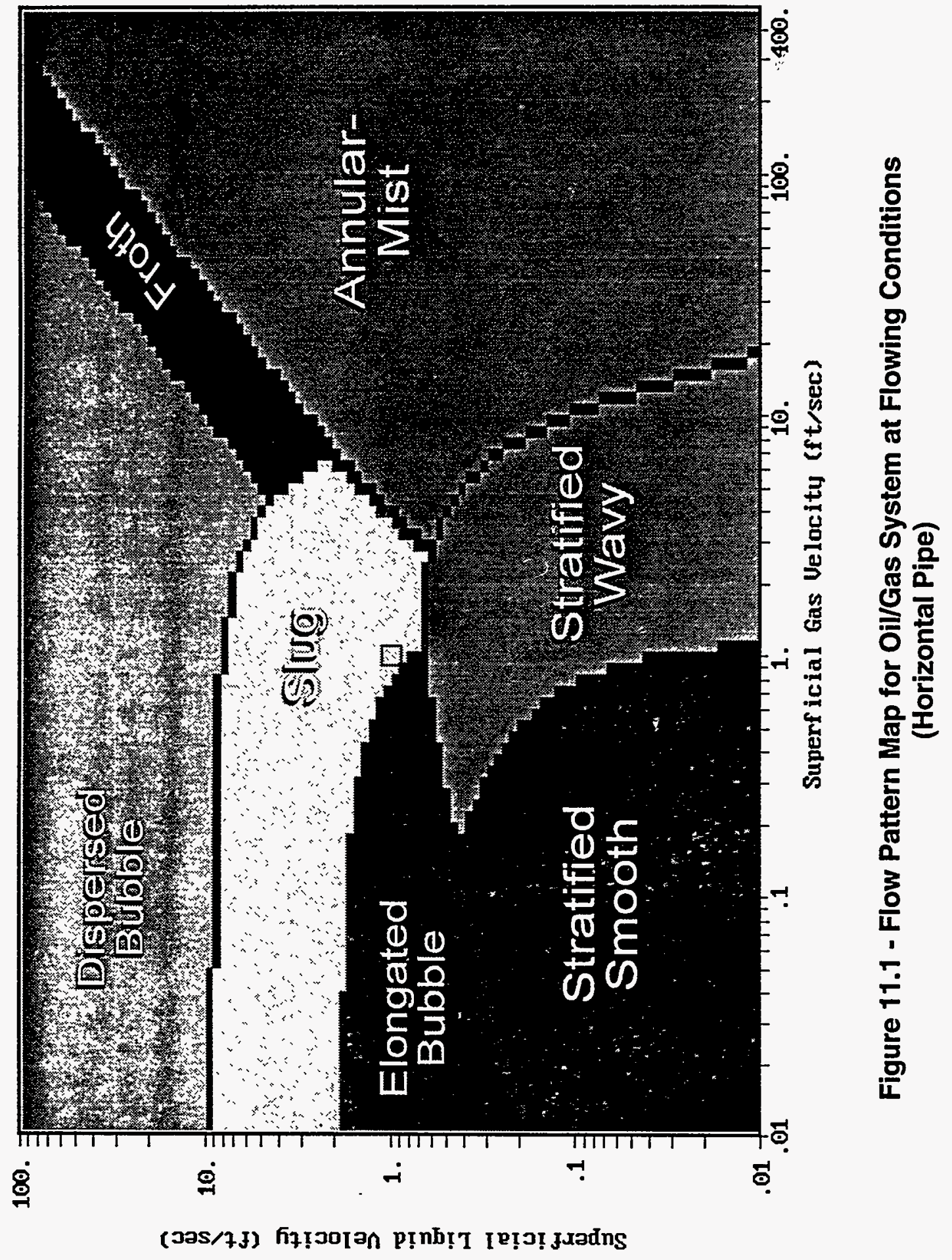




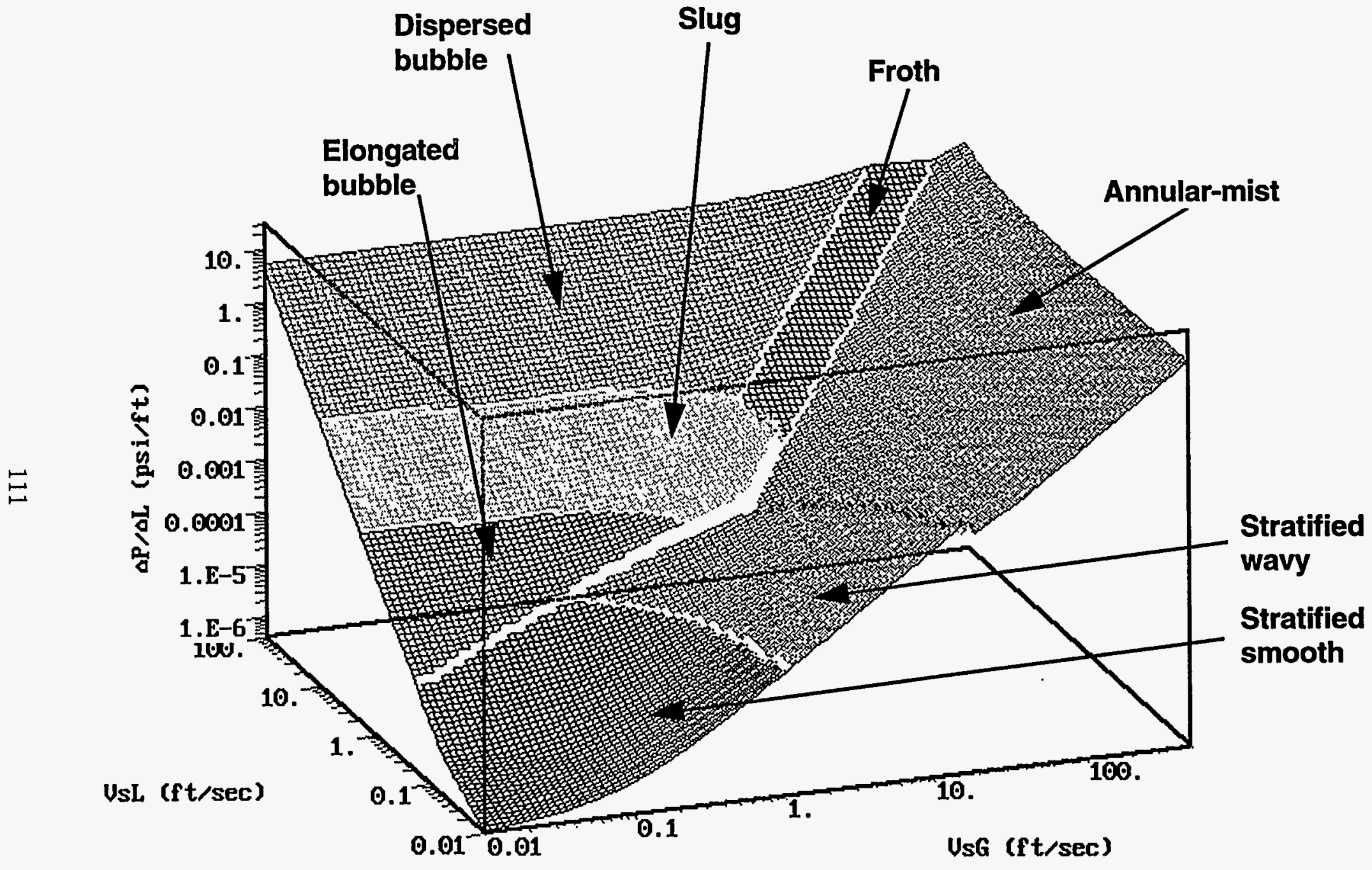

Figure 11.2 - Pressure Drop due to Friction for Oil/Gas System at Flowing Conditions (Horizontal Pipe) 


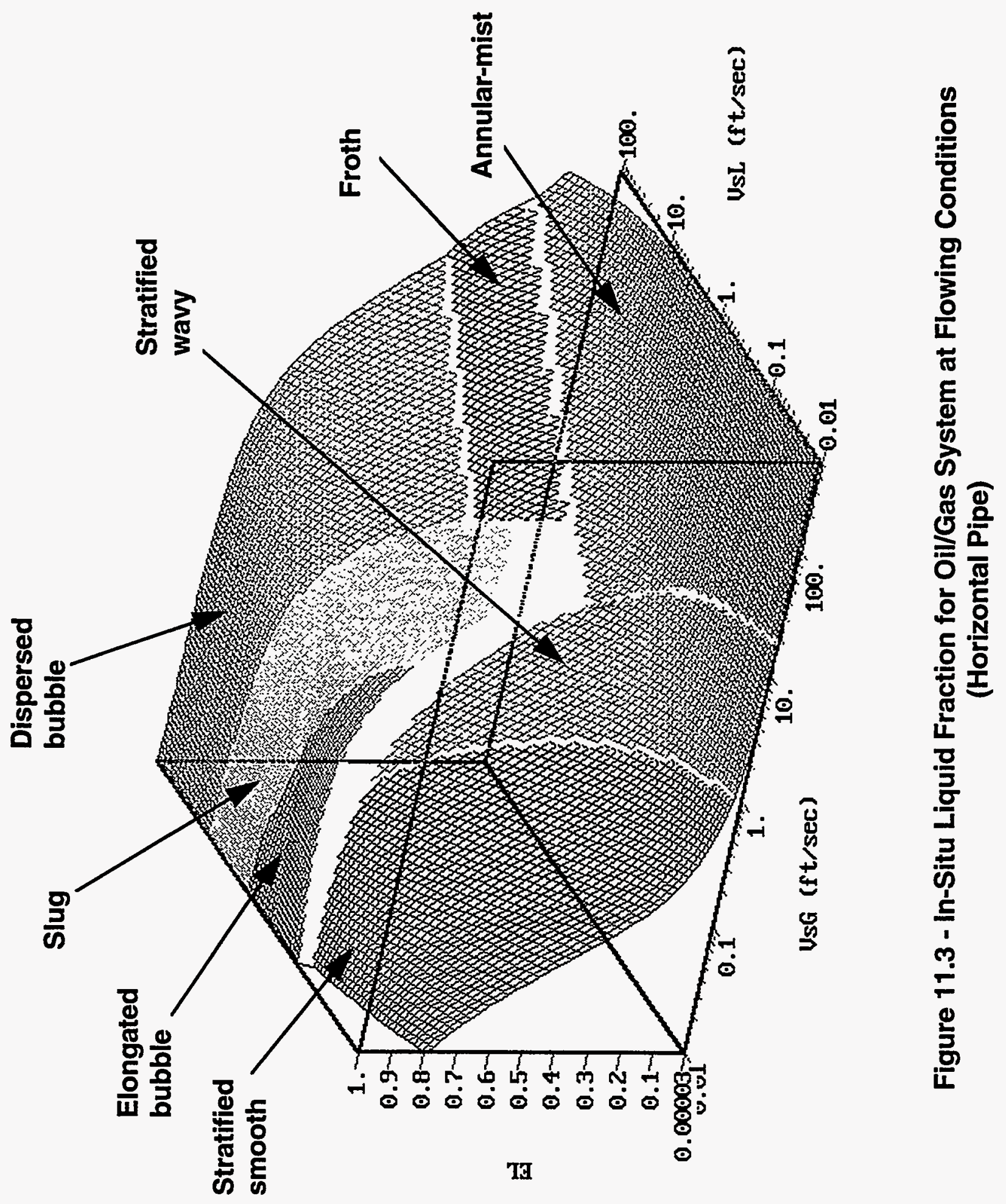




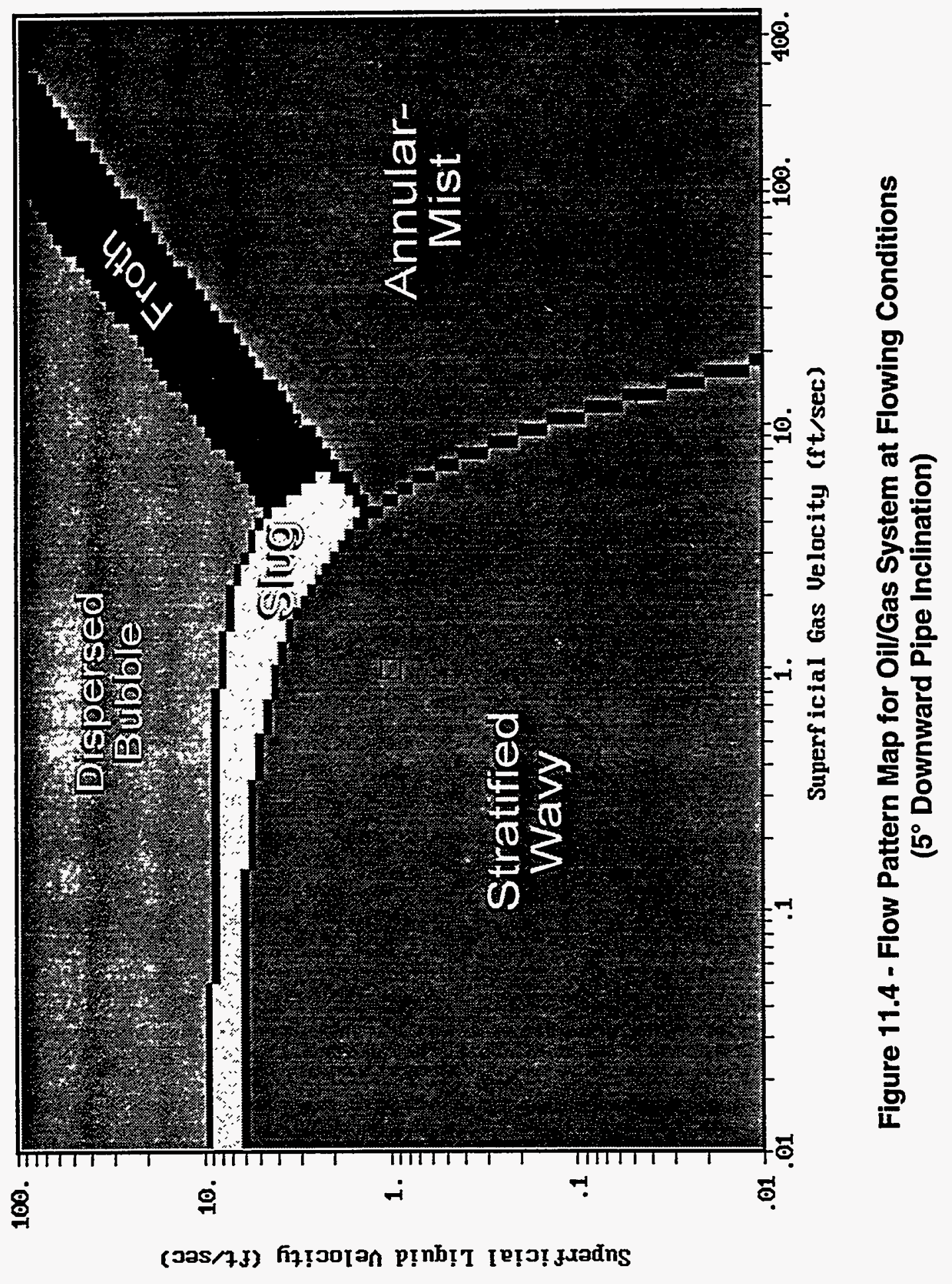




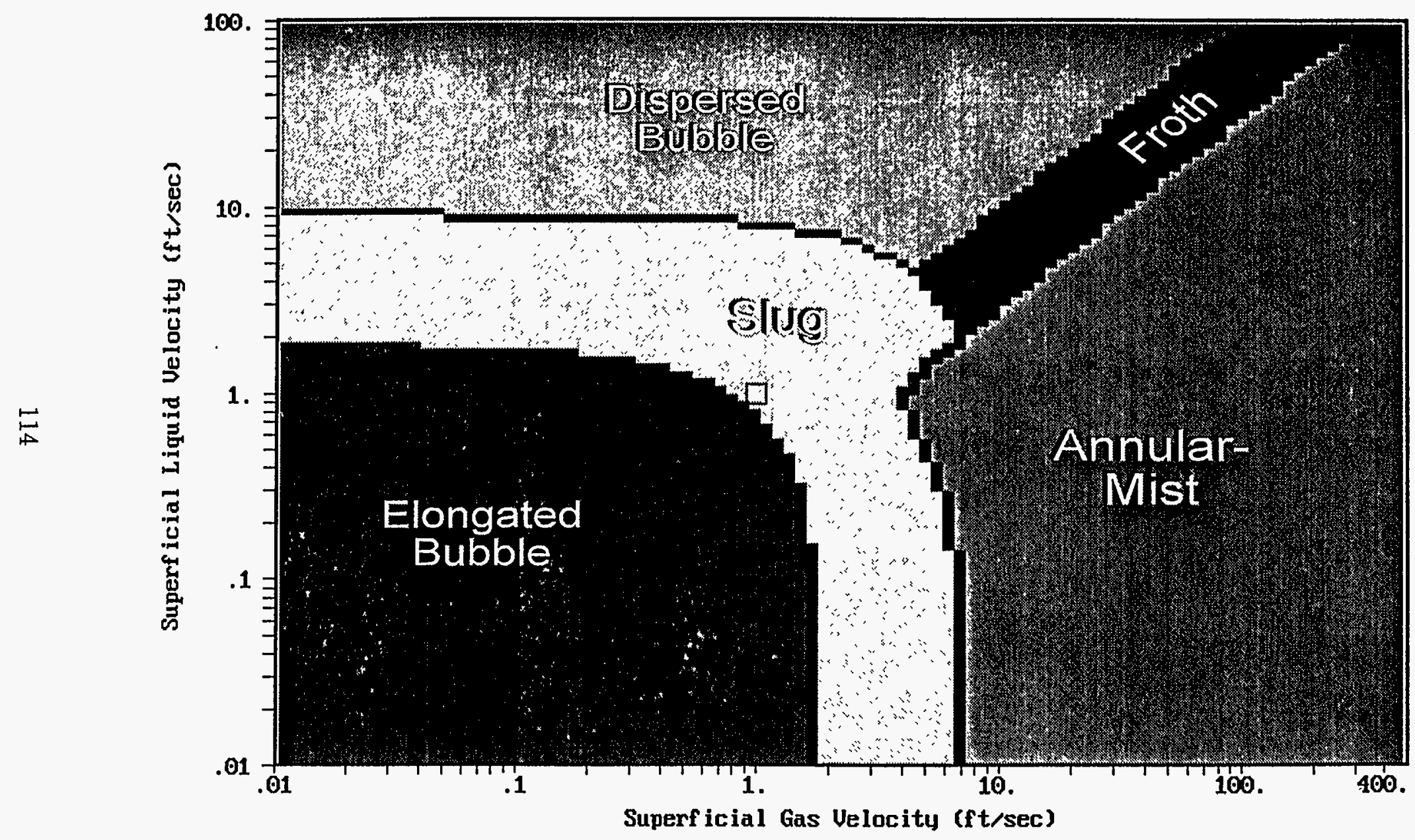

Figure 11.5 - Flow Pattern Map for Oil/Gas System at Flowing Conditions (5 Upward Pipe Inclination) 


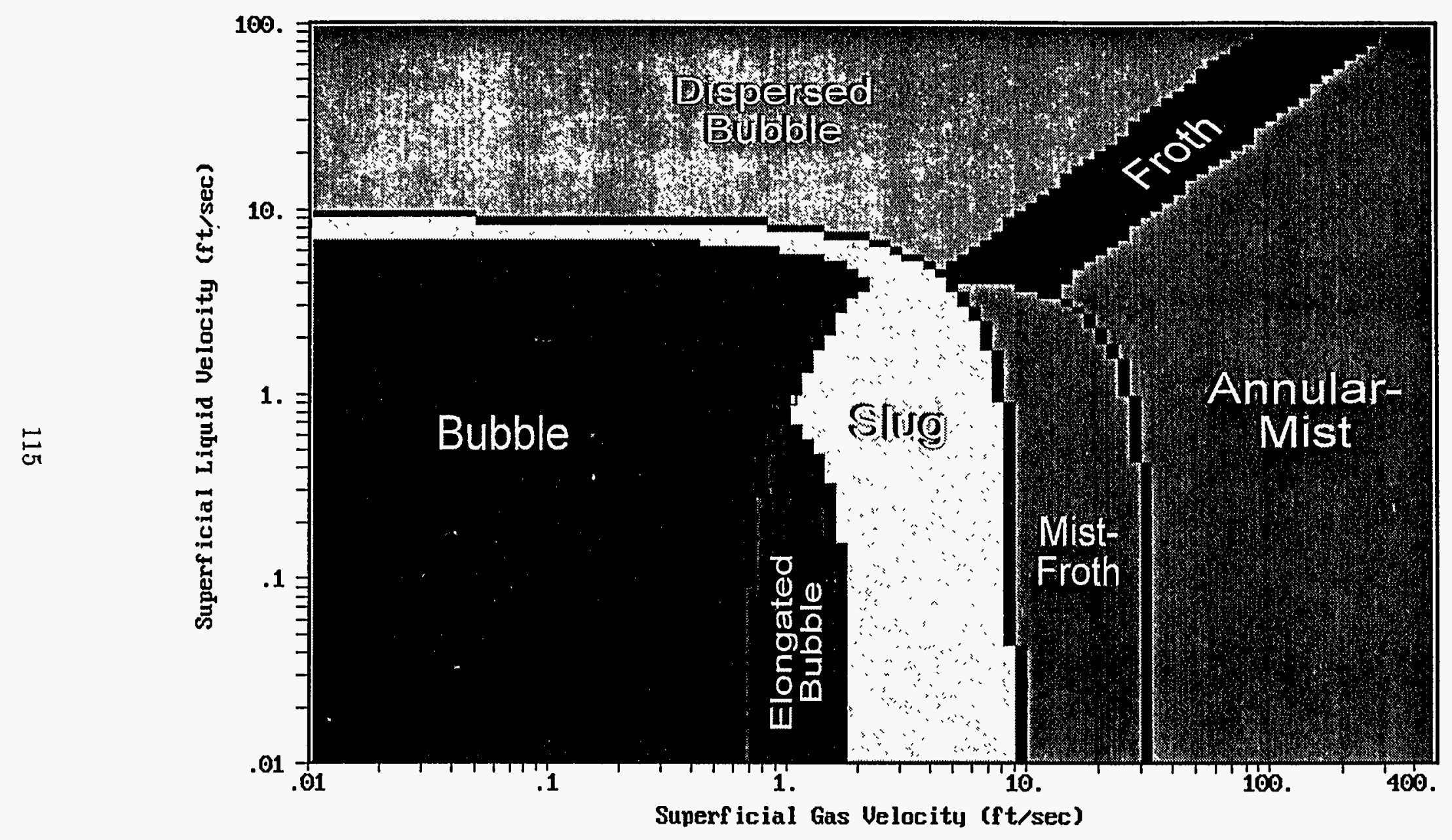

Figure 11.6 - Flow Pattern Map for Oil/Gas System at Flowing Conditions (80 ${ }^{\circ}$ Upward Pipe Inclination) 


\section{Experiments at Marathon Oil Company (Task 3)}

The major component of Task 3 involves planning and interpreting the measurements of two-phase flow pressure drops in experiments currently in progress at Marathon Petroleum Technology Center. The results will provide guidelines to (1) the accuracy of two-phase pressure drop correlations and (2) the effect of radial inflow on the flow patterns and their contribution to the total pressure loss in a $\mathrm{HW}$.

The Marathon Wellbore model is essentially a pipe with ID of 6.2 inches with feeder system for injecting flow radially through perforations. The acrylic pipe is transparent and flow patterns can be monitored with a moving TV-recording system. The layout consists of $20 \mathrm{ft}$ of blank acrylic, followed by $20 \mathrm{ft}$ of smooth perforations, followed by $15 \mathrm{ft}$ of sharp edged perforations. Liquid or gas inflow is supplied by the connected manifold to the next $40 \mathrm{ft}$ of smooth edged perforations. The remaining $5 \mathrm{ft}$ of the model is a blank section of acrylic casting. Fig. 12.1 displays a sketch of the layout. The rig can be tilted to various angles and therefore two-phase flow in inclined pipes can be studied.

Considerable modification and improvements to instrumentation have been necessary during 1993 to allow the wellbore model to be transformed from its previous role as a gravel packing experimental facility. A liquid holdup meter based on a capacitance measurement technique has been designed and calibrated for external mounting on the acrylic pipe. It is hoped to replicate this design during 1994. Pressure drop measurements for baseline experiments in single phase water or oil flows have necessitated the installation of and recording from liquid manometer gauges. For the larger pressure drops associated with two-phase flow, variable range Rosemount pressure transducers have been purchased. Funding of the changes to rig facilities has been provided by Marathon.

Fluid inflow through perforations may conceptually affect the flow resistance in the wellbore by (1) increasing the flow momentum via accelerating the inflow streams up to the average pipe flow velocity and (2) disturbing the boundary layer at the pipe wall and changing the wall friction. Both of these effects contribute to the total pressure drop along the wellbore. The second effect adds to the pressure loss due to the wall friction. In the following, we will report on the completed experiments and draw some conclusions.

\subsection{Completed Experiments and Data Analyses}

A number of experiments including single phase baseline runs and two-phase flow experiments have been completed. Water and oil are used as the liquid phase and the gas phase is either air or nitrogen. The purposes of performing single phase flow experiments are to (1) evaluate the working condition of the wellbore model and recognize the operating constraints of various components (e.g., pumps, pipes, gauges, etc.) of the flow loop and (2) characterize the wellbore model (i.e., obtain the actual roughness of the pipe) using the single-phase flow calculations. The Gould pump, specially installed in the model, can supply oil flow rates up to $500 \mathrm{gpm}$ and the current limit on the gas supply is about 0.5 MMscfd of vaporized liquid nitrogen, with similar capacity in the compressor available for air flows. Higher gas flow rates are possible but the handling capability of the current separator 


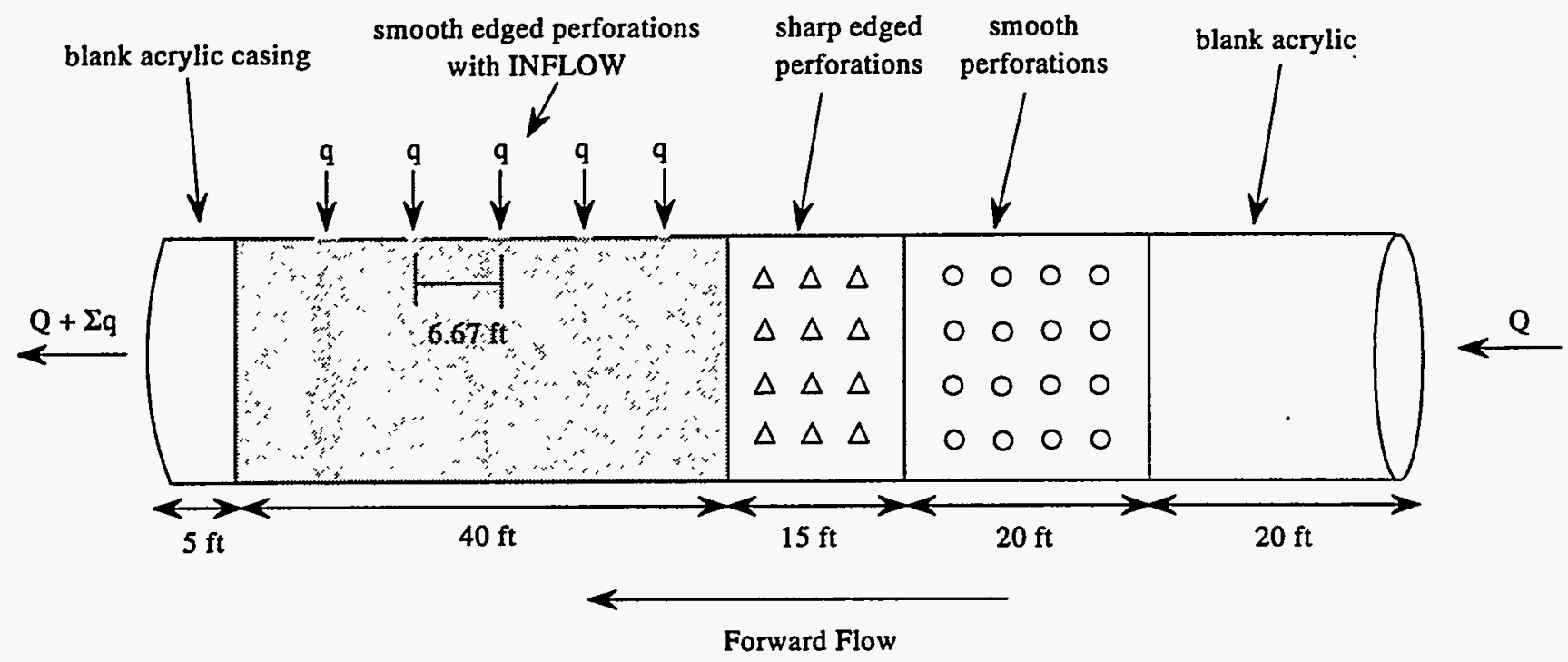

Figure 12.1: Layout of the Marathon Wellbore Model

prohibits such higher rates. The use of another separator with larger capacity is under study for the forthcoming high gas rate experiments.

The following experiments have been undertaken:

1. Single-phase water flow in the core with no inflow

2. Single-phase oil flow in the core with no inflow

3. Single-phase oil flow in the core with inflow of oil through perforations

4. Two-phase water/air flows with water in the core and air influx through perforations

5. Two-phase oil/nitrogen flows with oil in the core and influx of nitrogen through perforations

Each experiment is performed for a number of liquid and gas flow rates. The pressure drops for each $10 \mathrm{ft}$ section of the model (DP) is measured by liquid manometers. In the case of twophase experiments, DPs are also measured by sensitive pressure gauges for a more convenient non-steady measurement. The resulting flow patterns in the two-phase flow experiments are recorded by a scanning movie camera. Samples of measured data and analysis for each type of experiment are given in the next two sections starting with the single-phase experiments. All the computations of the DPs are done using the ASA software package [55].

\subsection{Single-Phase Flow Experiments}

The water core flow data are analyzed first. We have considered the data with water core flow rates of $Q=400,500$, and $580 \mathrm{gpm}$, in both forward and reverse directions (see Fig. 12.1). In the computation of the pressure drops, we have taken into account the measured 
variations of the wellbore diameter along the length of the model. For each $10 \mathrm{ft}$ section of the wellbore, an average diameter was calculated from the reported ultrasonic measurements of the diameter along the model. We first used the smooth pipe assumption and calculated the pressure drops per $10 \mathrm{ft}$ length of the pipe. Next we tried to match a single data point (the data for the 60-70 ft section with $Q=580 \mathrm{gpm}$ was used). A good match is obtained with a small absolute roughness of $\epsilon=8 \times 10^{-5} \mathrm{ft}$. The pressure drop computations are then performed with this value of roughness for all the cases. The data and results are shown in Figure 12.2. The DPs are placed at the middle of the sections to which they apply in the figure.

It can seen from Fig. 12.2 that the calculated "rough" case predicts the pressure drop data better than the calculated smooth case. In general the agreement between the data and the calculated values is good except for the last two sections in the forward flow direction (i.e., the 15-25 and 5-15 ft sections). The 15-25 ft section reads low in both directions for all flow rates while the 5-15 ft section reads very high especially for the reverse flow. The pressure drop in the 5-15 ft section is possibly influenced by the end or entrance effects in the forward or reverse flow directions respectively. An important feature is the observed consistency in the data. For instance, the same section reads low for all the flow rates in both directions. The sensitivity to flow direction which appears for the $5-15 \mathrm{ft}$ section is not seen at the opposite end (80-90 ft) even though the connection arrangements at each end are similar, i.e., 4 inch pipe joined to 6 inch test section. The reasons for this sensitivity at only one end are under investigation. Next, we examined the oil core flow data for flow rates of $Q=311,400$, and $493 \mathrm{gpm}$. These were performed in only one direction. Pressure drop calculations were made for the smooth pipe and the "rough" cases with the same roughness as used in the water case. Figure 12.3 displays the pressure drop data and the predictions. The higher oil viscosity results in lower Reynolds numbers than those of water $\left(R e \approx 4 \times 10^{4}\right.$ for oil versus $\approx 2 \times 10^{5}$ for water with $Q=400 \mathrm{gpm}$ ). Therefore, the pipe roughness and the variations of the diameter have a smaller effect on the pressure drops in oil runs in comparison with the water experiments as shown in Fig. 12.3.

The next analysis is also for the single-phase oil flow experiment but now some of the oil enters through perforations at the $45 \mathrm{ft}$ mark (measured from the left in Fig. 12.1). Figure 12.4 displays the data and the computations for the case with $Q=306 \mathrm{gpm}$ and $\sum q=85 \mathrm{gpm}$. The variations of the wellbore diameter are also taken into account. It can be seen from the Figure that an "effective" absolute roughness of $\epsilon=0.002 \mathrm{ft}$ predicts the DP data in the inflow section reasonably well. The data in the no-inflow section of the model is well represented by the smooth pipe calculations. There is no indication that the perforations, either smooth or sharp edge, effect the pressure drop when there is no flow through them. The smooth pipe predictions of DPs are nearly identical to the case with $\epsilon=8 \times 10^{-5} \mathrm{ft}$. This result indicates the importance of the effect of the inflow on changing the effective roughness of the pipe.

\subsection{Two-Phase Flow Experiments}

Figure 12.5 is for the case where water enters the wellbore from the right (at the 100 $\mathrm{ft}$ mark) and air is supplied ( $\left.\sum q=215 \mathrm{scfm}\right)$ in the radial direction starting from the $45 \mathrm{ft}$ 


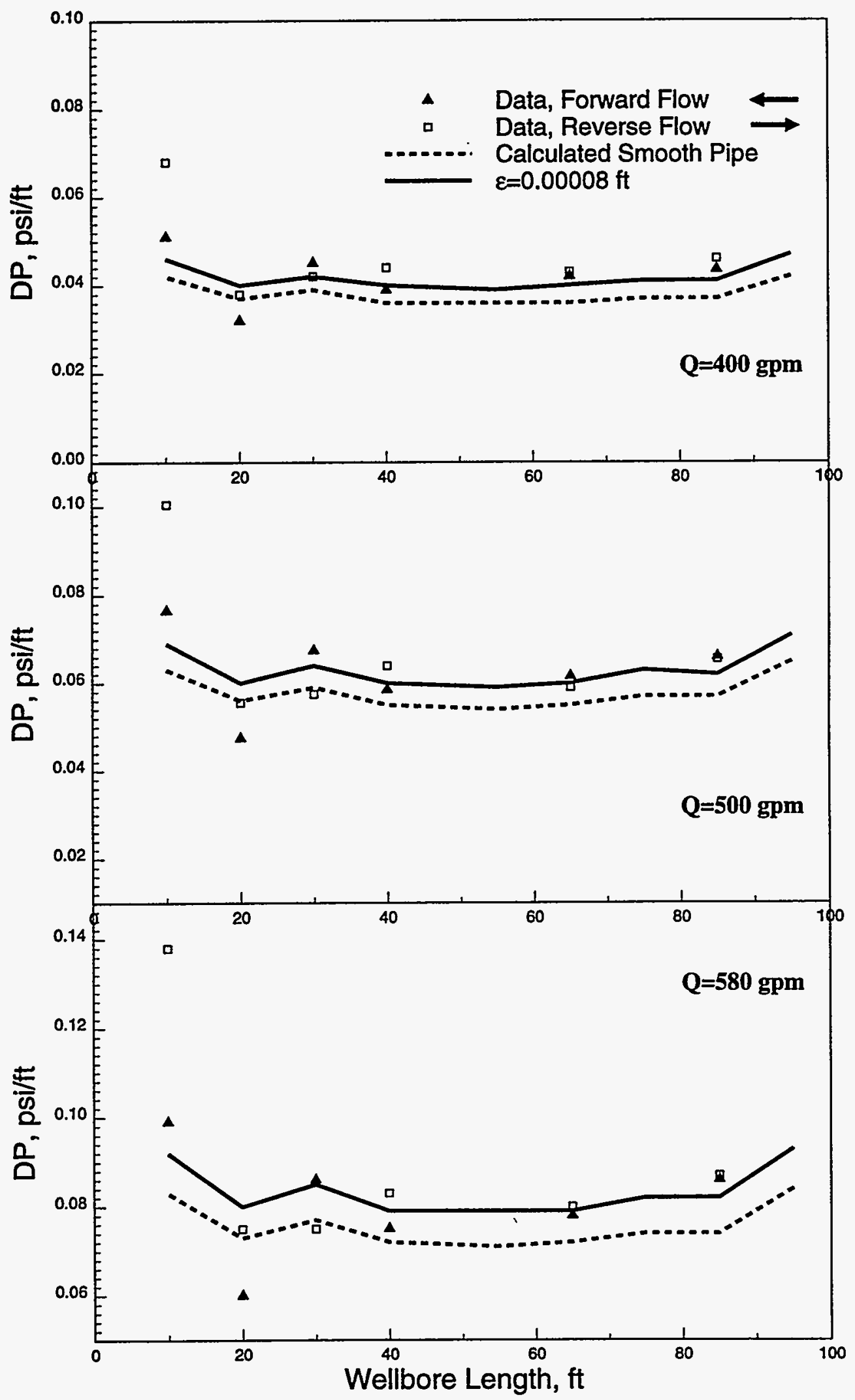

Figure 12.2: Data Analysis of Single-Phase Water Core Flow Experiments 


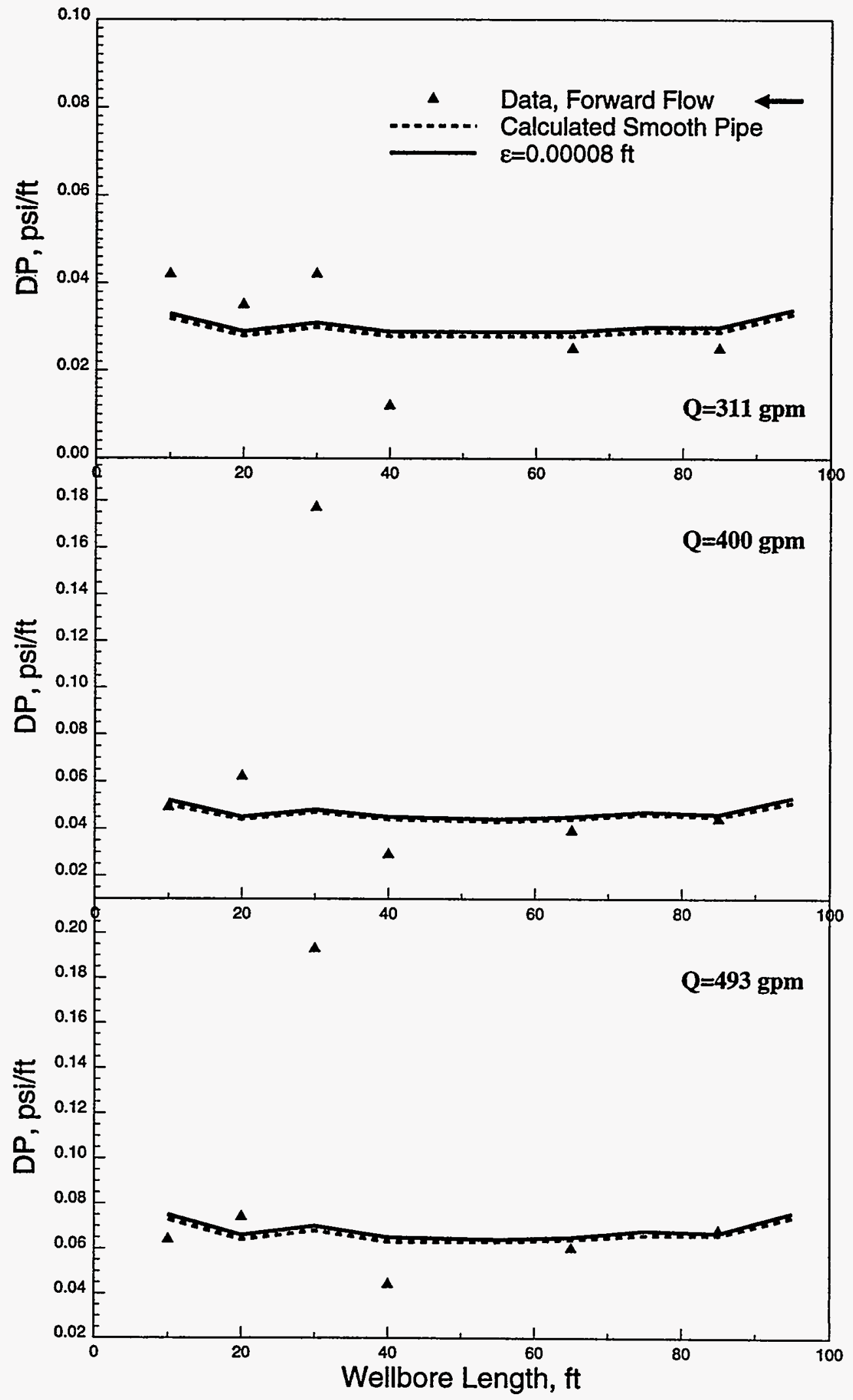

Figure 12.3: Data Analysis of Single-Phase Oil Core Flow Experiments 


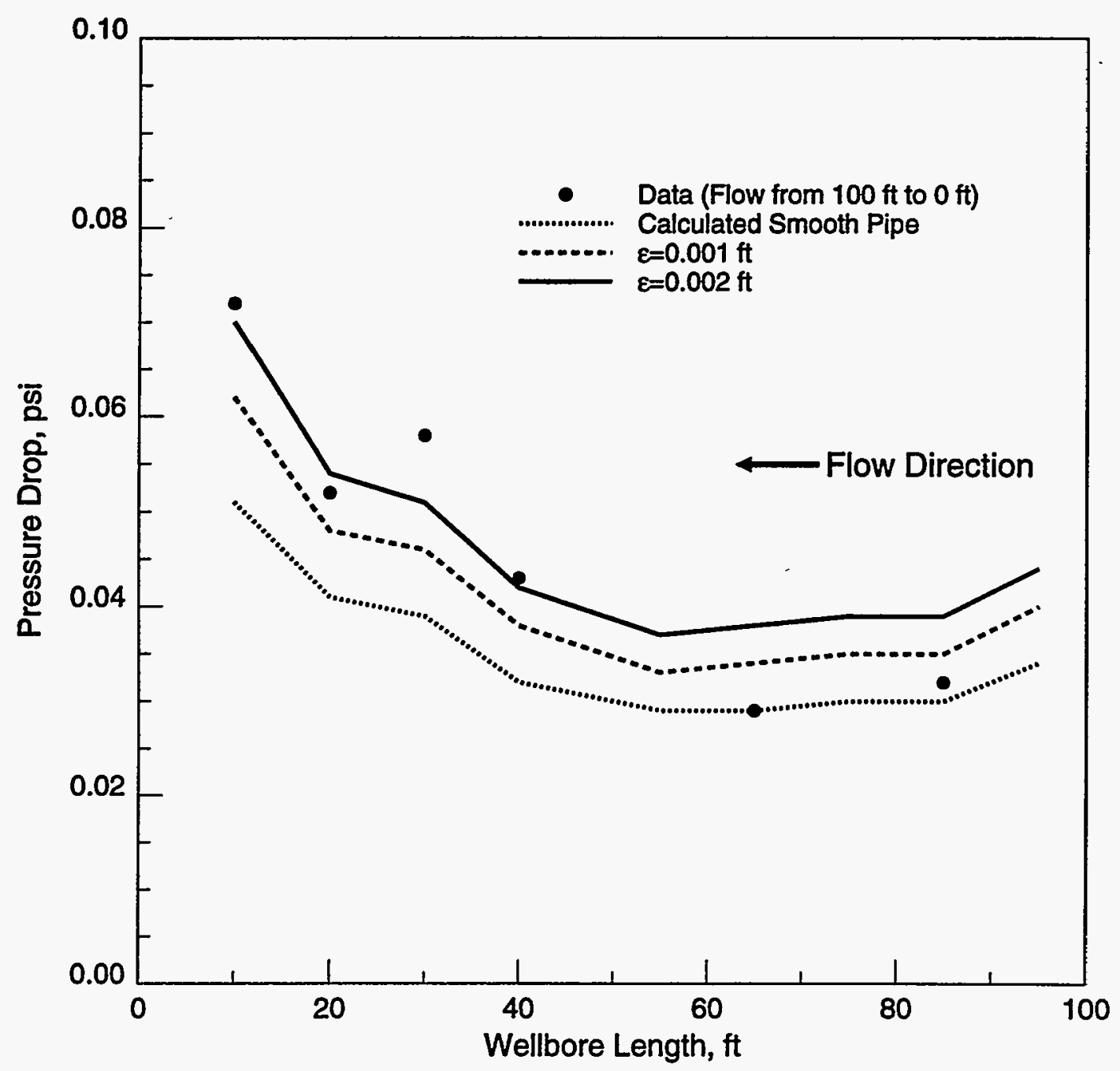

Figure 12.4: Data Analysis of Single-Phase Oil Flow Experiments with Oil in the Core $(Q=306 \mathrm{gpm})$ and through Perforations $\left(\sum q=85 \mathrm{gpm}\right)$

mark. The resulting two-phase flow causes a larger pressure drop as the data indicate. The lines in Fig. 12.5 show predictions of two-phase pressure drops computed from the empirical correlations of Beggs and Brill [5] (with roughness of $\epsilon=0.001 \mathrm{ft}$ ) and those of Dukler et al. [56] (with $\epsilon=0.001$ and $0.002 \mathrm{ft}$ ). The results in Fig. 12.5 indicate the need to use larger roughness (or friction factors) to predict the two-phase flow pressure drops. A value of roughness between 0.001 and $0.002 \mathrm{ft}$ seems plausible. Note that the variations in the wellbore diameter are not taken into account in the calculations of the results in Fig. 12.5. Finally, the last case is for the two-phase flow experiment of oil and nitrogen. The core oil flow rate is $Q=308 \mathrm{gpm}$ with the total nitrogen influx rate of $\sum q=0.415$ MMscfd (288 $\mathrm{scfm}$ ). Figure 12.6 shows the data and the predictions of the correlations. It can be observed that again a higher "effective" roughness $(\epsilon=0.002 \mathrm{ft})$ is needed to obtain a reasonable match with the data in the two-phase flow section. The DPs in the single phase region (from the 100 to the 45 mark in Fig. 12.1) in Fig. 12.6 are smaller than the corresponding data 


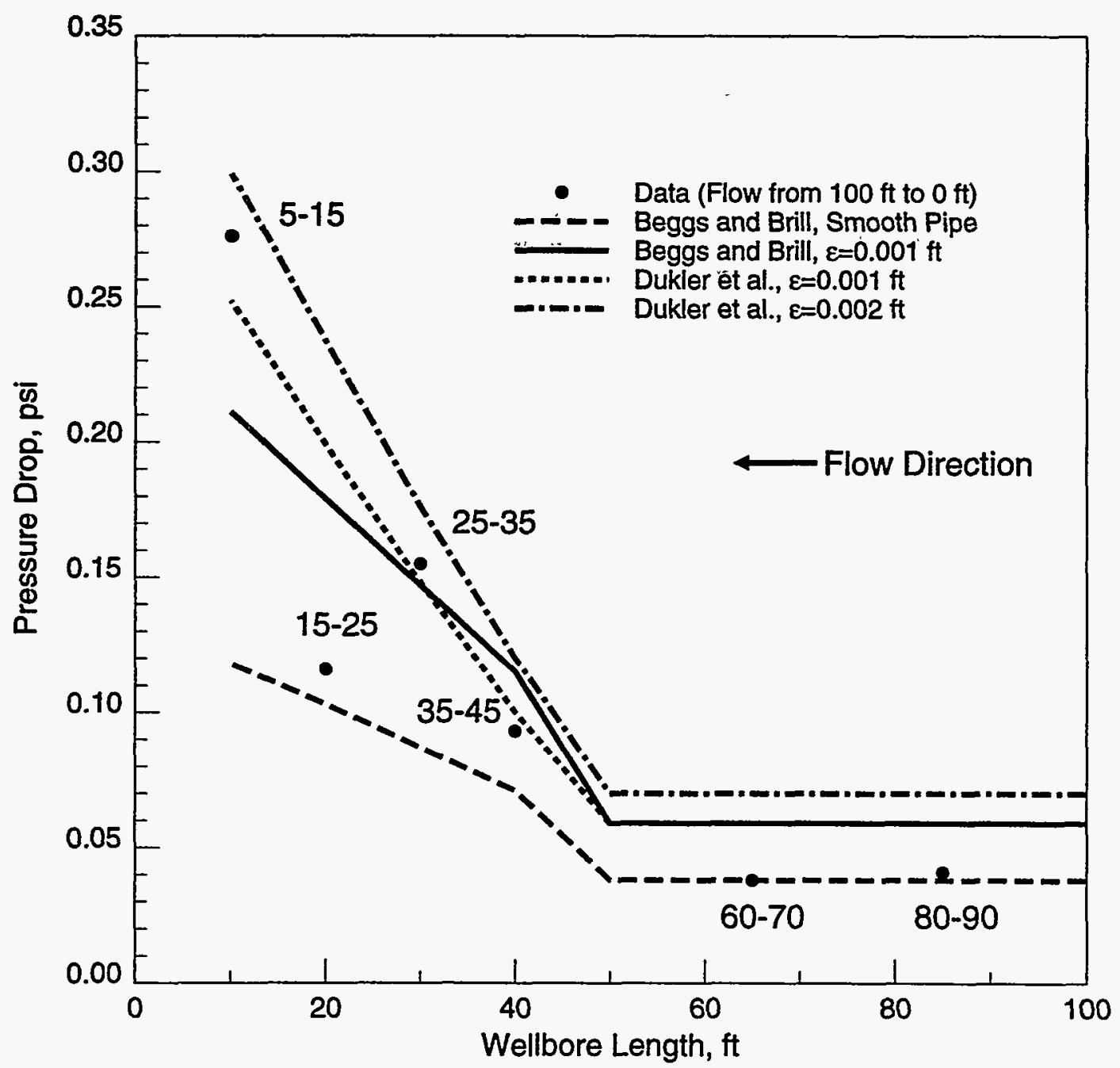

Figure 12.5: Pressure Drop Along the Wellbore for $Q=400 \mathrm{gpm}$ (Water, core flow), $\sum q=215 \operatorname{scfm}$ (Air, inflow)

in Fig. 12.5 due to the reduced liquid velocity. The larger two-phase pressure drop can be attributed to the higher gas flow rate of $288 \mathrm{scfm}$ in this experiment in comparison with the rate of $215 \mathrm{scfm}$ in the previous run.

The position of the new liquid holdup meter is shown in the photograph of the 5$15 \mathrm{ft}$ section of the acrylic pipe in Fig. 12.7. A liquid slug moving from right to left has just passed through the meter. The response of the capacitance meter to passage of liquid slugs in this section is shown in Fig. 12.8, and corresponding measurements of DP from the pressure transducers is shown in Fig. 12.9. Work on interpreting these data will form pat of the future research activity.

\subsection{Remarks and Future Plans}

We may compile a number of conclusions based on the performed experiments. 


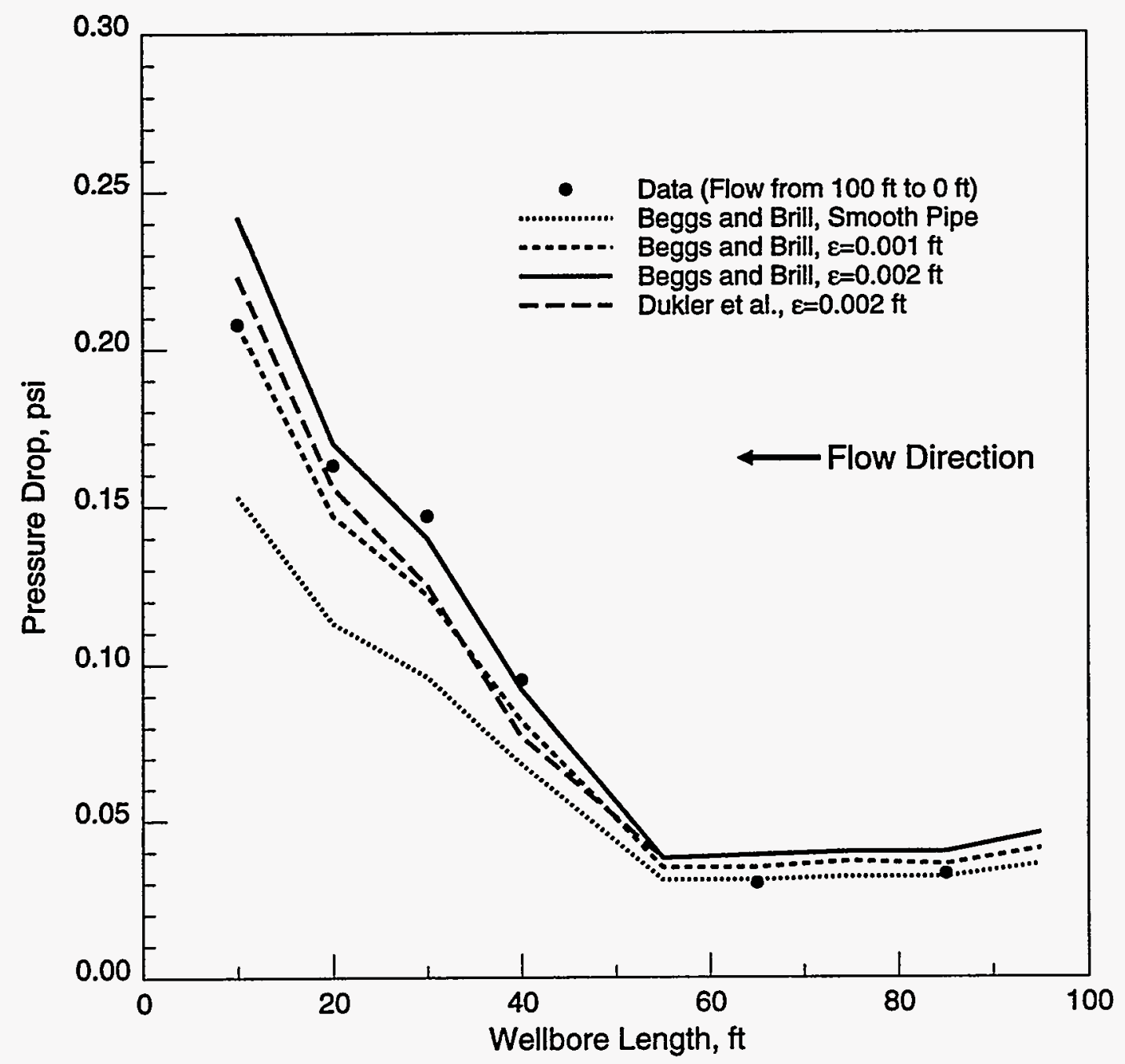

Figure 12.6: Pressure Drop Along the Wellbore for $Q=308 \mathrm{gpm}$ (Oil, core flow), $\sum q=288$ scfm (Nitrogen, inflow)

1. The single phase core flow data indicate that the wellbore can be characterized as essentially a smooth pipe. Pressure drops for these experiments are quite small. The effects of various perforation types in the model on the DPs are not apparent in the measurements, when there is no inflow through the perforations.

2. The single phase experiments involving core and radial influx flows show larger pressure drops in the inflow section. The difference is caused by both the momentum changes and the effects of radial flow disturbing the boundary layer.

3. The two phase flow data indicate even larger pressure drops than the single-phase with influx data. An "effective" roughness of 0.001 to $0.002 \mathrm{ft}$ is required to reasonably match the data with the standard correlation methods. The magnitude of the pressure drop is mainly controlled by the gas flow rate. 
In recognition of the last remark, we plan to run experiments with higher gas flow rates of up to $2 \mathrm{MMscfd}$. In addition to installing a new separator, this involves a number of other changes to the rig. Liquid holdups will be measured at minimum three locations. A prototype device for measuring liquid holdups has been successfully tested. The experimental data will be used in evaluating and improving the developed mechanistic model discussed in chapter 11 . 


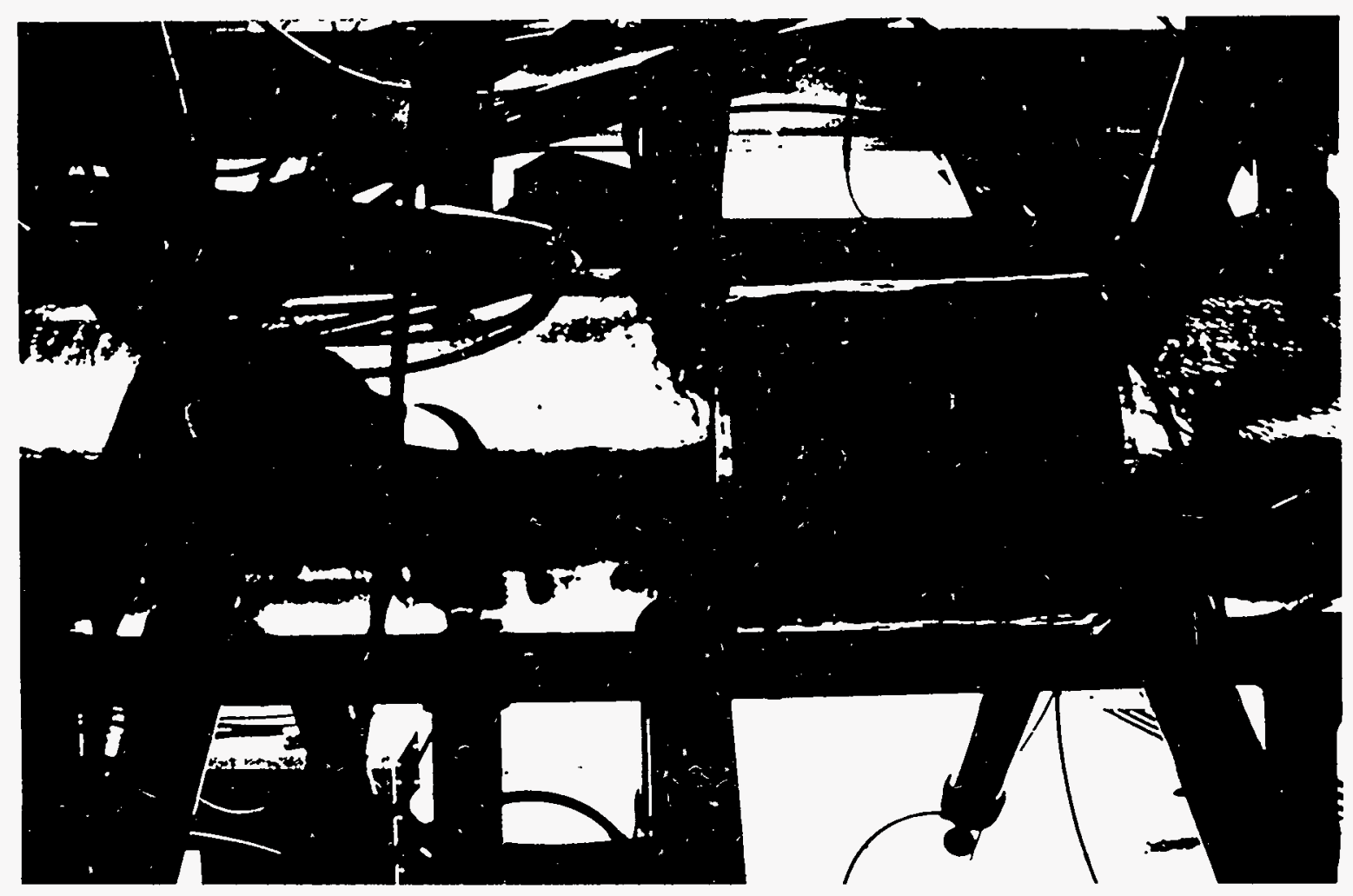

Figure 12.7: Photograph of Liquid Holdup Meter in Position in the 5-15 ft section of Acrylic Pipe 


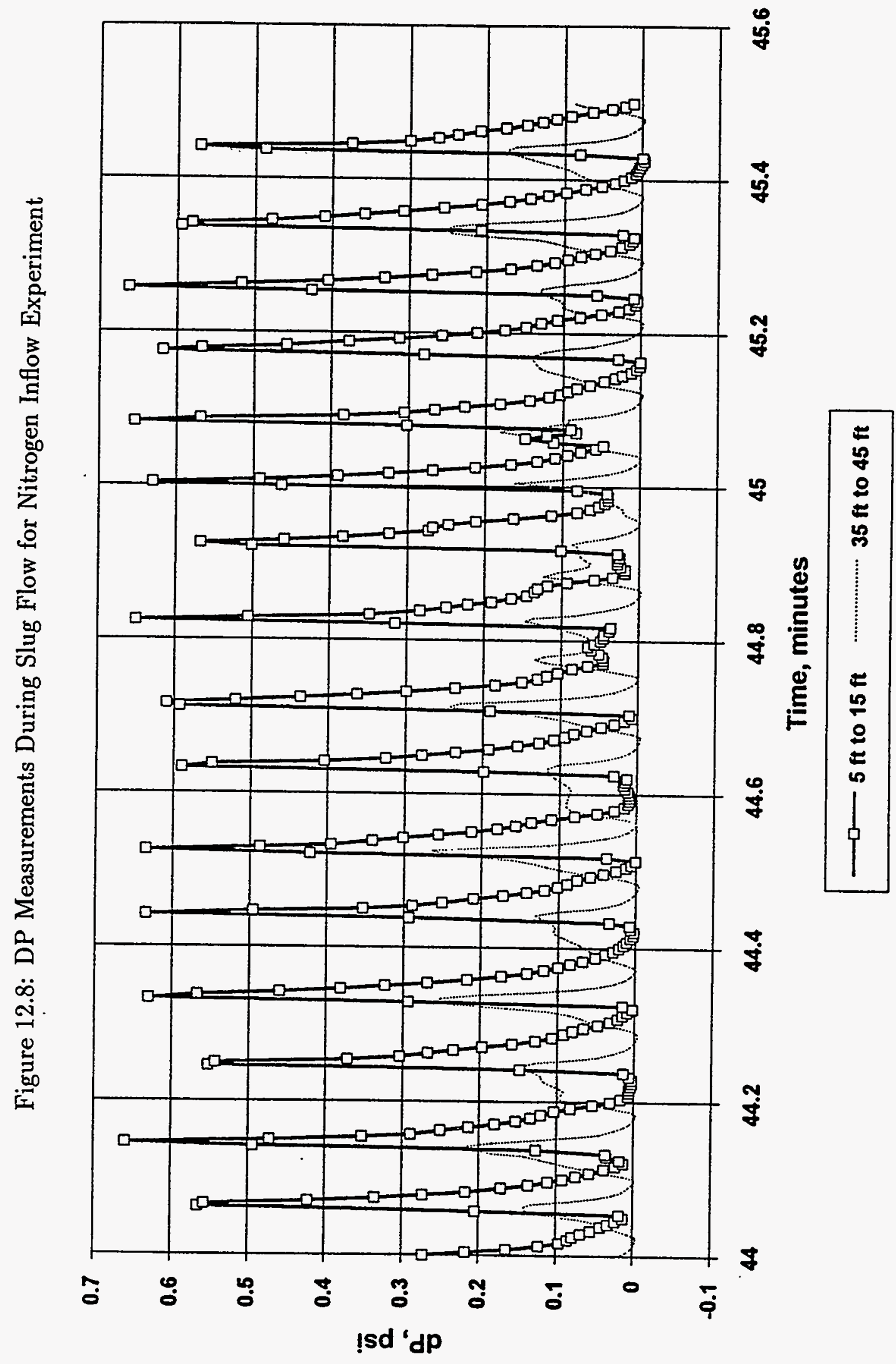




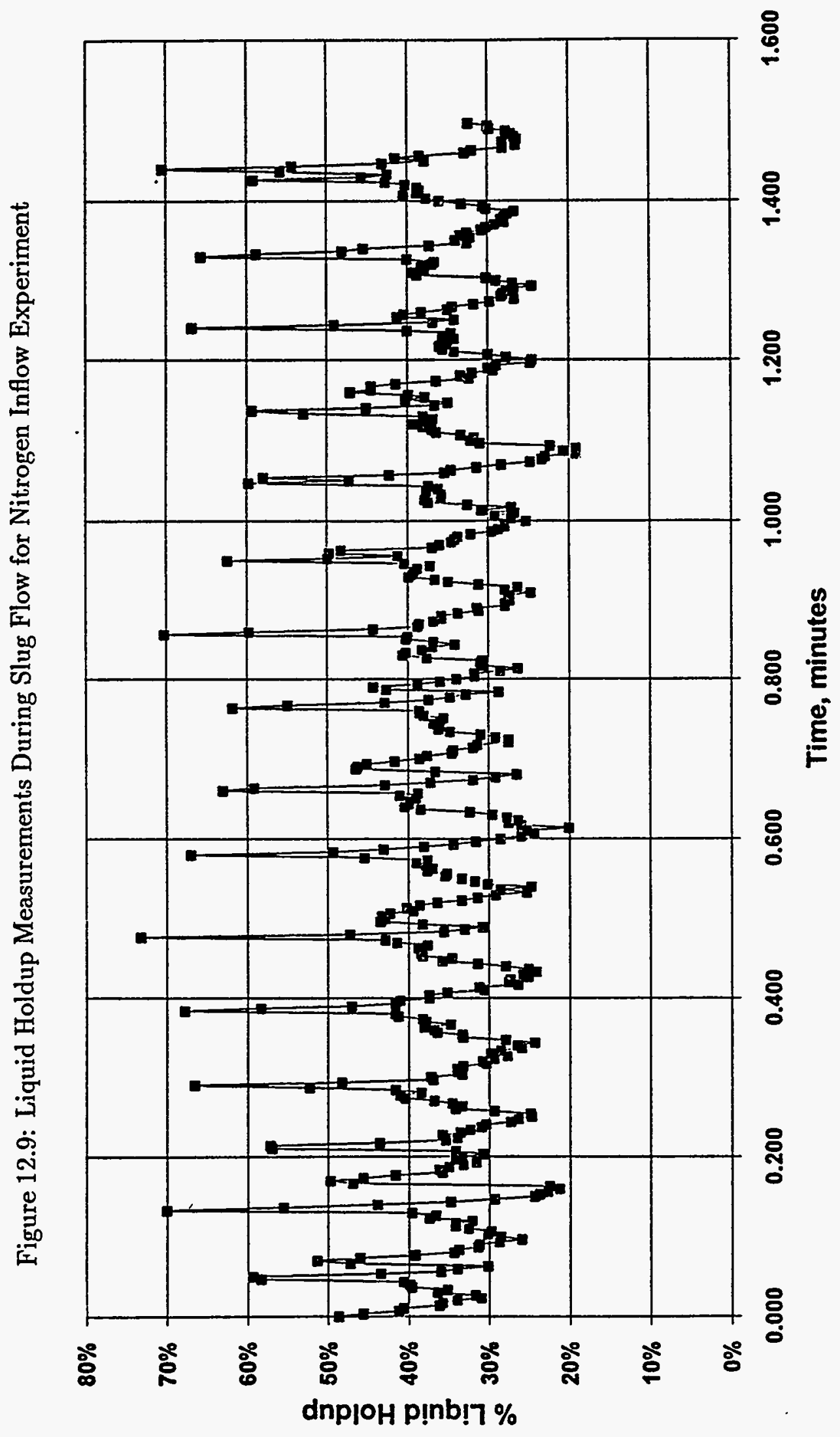




\section{Nomenclature}

$\begin{array}{ll}a & \text { Line source strength } \\ b & \text { Point source strength } \\ B_{o} & \text { Oil formation volume factor } \\ \triangle i & \text { Length of Grid block in the } i^{t h} \text { direction } \\ f & \text { Fanning friction factor } \\ f_{c} & \text { Transmissivity correction factor } \\ g & \text { Gravitational acceleration } \\ i_{w}, j_{w} & \text { Integer coordinates of well location } \\ h & \text { Pay zone thickness (height of the reservoir) } \\ H_{d} & \text { Critical crest height } \\ k_{i} & \text { Permeability in the } i^{t h} \text { direction } \\ n_{i} & \text { Number of grid blocks in the } i^{t h} \text { direction } \\ p & \text { Pressure } \\ P_{w} & \text { Well pressure } \\ P_{o} & \text { Well block/grid block pressure } \\ P_{D} & \text { Dimensionless pressure } \\ q_{c} & \text { Critical oil flow rate } \\ q_{D C} & \text { Dimensionless critical rate } \\ Q_{o, c} & \text { Total critical oil flow rate for a horizontal well } \\ Q_{o, v} & \text { Total critical oil flow rate for a vertical well } \\ q & \text { Flow rate } \\ r_{w} & \text { Well radius } \\ r_{o} & \text { Effective radius } \\ R e & \text { Reynolds number } \\ r_{e} & \text { Effective radius of a well/well block } \\ r_{c} & \text { Equivalent radius of a block } \\ r_{e h} & \text { Effective drainage radius } \\ S & \text { Skin factor } \\ t & \text { Time } \\ t_{B t} & \text { Breakthrough time } \\ t_{D} & \text { Dimensionless time } \\ & \\ & \end{array}$


$T_{i j} \quad$ Transmissivity between points $i$ and $j$

$x_{e} \quad$ Longitudinal well spacing

$x_{w} \quad x$ coordinate location of the well

$X_{D} \quad$ Well completion penetration fraction

$y_{e} \quad$ Transverse well spacing

$y_{w} \quad y$ coordinate location of the well

$Y_{D} \quad$ Dimensionless depth of the line source

$Z_{S} \quad$ Dimensionless depth to the apex of the cone

$z_{w} \quad$ Height of well above the bottom of pay zone

\section{Greek Letters}

$\alpha_{g, 0} \quad$ Volume fractions of gas and oil respectively

$\beta \quad$ Anisotropy scale factor

$\epsilon \quad$ Absolute roughness

$\mu_{0} \quad$ Oil viscosity

$\omega \quad$ Well direction angle relative to $z$-axis

$\phi \quad$ Porosity

$\Phi$ Oil potential function

$\rho \quad$ Density

$\theta \quad$ Well direction angle relative to $\mathrm{x}$-axis

\section{Subscript}

$D$ Dimensionless parameter

\section{Superscript}

- Spatially transformed property

- Effective property 


\section{References}

[1] Moritis, G. : "Horizontal Drilling Technology Keeps Advancing," Oil \& Gas Journal Special, (March 11, 1991) 49-54.

[2] Reisz, M. R. : discussion following "Reservoir Evaluation of Horizontal Bakken Well Performance on the Southwestern Flank of the Williston Basin," paper SPE 22389 presented at the 1992 SPE International Meeting on Petroleum Engineering, Beijing, March.

[3] Koonsman, T. L. and A. J. Purpich : "Ness Horizontal-Well Case Study," J. Pet. Tech., (October 1992) 1066-1072.

[4] Sherrard D. W., Brice B. W. and D. G. MacDonald : "Application of Horizontal Wells at Prudhoe Bay," J. Pet. Tech., (November 1987) 1417-1425.

[5] Beggs, H. D. and J. P. Brill : "A Study of Two Phase Flow in Inclined Pipes," J. Pet. Tech., (May 1973) 25, 607-617.

[6] Haug, B. T. : "The Second Long-Term Horizontal Well Test in Troll: Successful Production From a 13-m Oil Column with the Well Partly Completed in the Water Zone," paper SPE 24943 presented at the 1992 SPE Annual Technical Conference and Exhibition, Washington D.C., October.

[7] Huang, W-S., Kaetzer, T. R., and K. R. Bowlin : "Design and Performance of a Horizontal Well Waterflood Project in New Hope Shallow Unit, Franklin County, Texas," paper SPE 24940 presented at the 1992 SPE Annual Technical Conference and Exhibition, Washington D.C., October.

[8] Shiekholeslami, B. A., Schlottman, B. W., Seidel, F. A., and D. M. Button : "Drilling and Production Aspects of Horizontal Wells in the Austin Chalk," J. Pet. Tech., (July 1991) 43, 773-779.

[9] Liboriussen, J. R. : "Horizontal Wells with Multiple Hydraulic Fractures," Thirteenth World Petroleum Congress on Reservoir Management and Improved Recovery (IOR), Buenos Aires, 1991.

[10] Moritis, G. : "EOR Increases 24\% Worldwide; Claims $10 \%$ of U.S. Production," Oil and Gas Journal Special, (April 1992) 51-79.

[11] Butler, R. M. and D. J. Stephens : "The Gravity Drainage of Steam-Heated Heavy Oil to Parallel Horizontal Wells," J. Can. Pet. Tech., (June 1981) 20, 90-96. 
[12] Sahuquet, B. C., Spreux, A. M., Corre, B., and M. P. Guitturd : "Steam Injection in a Low Permeability Reservoir through a Horizontal Well in Lacq Superieur Field," paper SPE 20526 presented at the 1990 SPE Annual Technical Conference and Exhibition, New Orleans, September.

[13] Peaceman, D. W. : "Interpretation of Well-Block Pressures in Numerical Reservoir Simulation with Nonsquare Grid Blocks and Anisotropic Permeability," Soc. Pet. Eng. J. (June 1983) 531-543.

[14] Babu, D. K., Odeh, A. S., Al-Khalifa A-J., and R. C. McCann: "The Relation Between Wellblock and Wellbore Pressure in Numerical Simulation of Horizontal Wells," SPE Reservoir Engineering (August 1991) 324-328.

[15] Brigham, W. E.: "Discussion of Productivity of a Horizontal Well," SPE Reservoir Engineering, (May 1990) 254-255.

[16] Nacul, E. C. and K. Aziz: "Use of Irregular Grid in Reservoir Simulation," paper SPE 22886 presented at the 1991 SPE Annual Technical Conference and Exhibition, Dallas, Texas, October.

[17] Pedrosa, Jr., O. A. and K. Aziz: "Use of Hybrid Grid in Reservoir Simulation," SPE Reservoir Engineering, (November 1986) 611-621.

[18] Palagi, C. L., Generation and Application of Voronoi Grid to Model Flow in Heterogeneous Reservoirs, Ph.D. Dissertation, Stanford University, May 1992.

[19] Economides, M. J., Diembacher F. X., Brand C. W., and Z. E. Heinemann : "Comprehensive Simulation of Horizontal Well Performance," SPE Formation Evaluation, (December 1991) 418-426.

[20] Palagi, C. L. and K. Aziz: "Use of Voronoi Grid in Reservoir Simulation," paper SPE 22889 presented at the 1991 SPE Annual Technical Conference and Exhibition, Dallas, Texas, October.

[21] Giger, F.: "Reduction du nombre de puits par l'utilisation de forages horizontaux," Revue de l'Institut Francais du Petrole, Vol. 38, No. 3, May-June 1983.

[22] Joshi, S. D. : "Augmentation of Well Productivity with Slant and Horizontal Wells," J. Pet. Tech., (June 1988) 729-739.

[23] Joshi, S. D., Horizontal Well Technology, Pennwell books, Oklahoma (1991) 288-292.

[24] Babu, D. K. and A. S. Odeh: "Productivity of a Horizontal Well: Appendices A and B,"paper SPE 18334 available from SPE Book Order Dept., Richardson, TX.

[25] Babu, D. K. and A. S. Odeh: "Productivity of a Horizontal Well," SPE Reservoir Engineering, (November 1989) 417-421.

[26] Goode, P. A. and F. J. Kuchuk: "Inflow Performance of Horizontal Wells," SPE Reservoir Engineering, (August 1991) 319-323. 
[27] Goode, P. A. and D. J. Wilkinson: "Inflow Performance of Partially Open Horizontal Wells," J. Pet. Tech., (August 1991) 983-987.

[28] Mclennan, J. D., Roegiers, J-C., and M. J. Economides : "Extended Reach and Horizontal Wells," Reservoir Stimulation, M. J. Economides and K. G. Nolte (eds.), Second Edition, Prentice Hall, Inc., Englewood Cliffs, NJ (1989).

[29] Consonni, P., Thiele, M., Palagi, C., and K. Aziz : "Flexible Gridding Techniques for Coning Studies in Vertical and Horizontal Wells," paper SPE 25563 presented at the SPE Middle East Oil Show, April 3-6 1993.

[30] Aziz, K.: Notes for Petroleum Reservoir Simulation,, Stanford University, Stanford (1993).

[31] Peaceman, D. W.: "Interpretation of Well-Block Pressures in Numerical Reservoir Simulation," Soc. Pet. Eng. J. (June 1978) 183-194; Trans. AIME, 265.

[32] Pedrosa, O.A.: Use of Hybrid Grid in Reservoir Simulation, Ph.D. Dissertation, Stanford University, December 1984.

[33] Bird, R.B., Stewart, W.E. and E. N. Lightfoot: Transport Phenomena, John Wiley \& Sons, New York (1960)

[34] Mucke, Ernst: Detri, 3-D Delaunay Triangulation Program, University of Illinois at Urbana-Champaign.

[35] Avis D. and B. K. Bhattacharya: Algorithms for computing $d$-dimensional Voronoi diagrams and their duals, in: F.P. Preprate ed., Advances in Computing Research (1983), 1, JAI Press,159-180.

[36] Phillips, Mark: Geomview (1993), The Geometry Center, University of Minnesota.

[37] Lien, S. C., Haldorsen, H. H., and M. Manner: "Horizontal Wells: Still Appealing in Formations with Discontinuous Permeability Barriers?," J. Pet. Tech. (December 1992) 1364-1370.

[38] Begg, S. H. and P. R. King: "Modeling the Effects of Shales on Reservoir Performance: Calculation of Effective Vertical Permeability,"paper SPE 13529 presented at the 1985 SPE Reservoir Simulation Symp., Dallas, Texas, February.

[39] Desbarats, A. J.: "Numerical Estimation of Effective Permeability in Sand-Shale Formations," Water Resources Research, (February 1987) 23: 273-286.

[40] King, P. R.: "The Use of Renormalization for Calculating Effective Permeability," Trans. Porous Media, (1989) 4:37-58.

[41] Giger, F. M. : "Evaluation theorique de l'effet d'arete d'eau sur la production par horizontaux," Revue de l'Institut Francais du Petrole, (May-June 1983) 38 No. 3 (in French). 
[42] Giger, F. M. : "Analytic 2-D Models of Water Cresting before Breakthrough for Horizontal Wells," SPE Reservoir Engineering, (November 1989) 409-416.

[43] Karcher, B. J., Giger, F. M., and J. Combe : "Some Practical Formulas to Predict Horizontal Well Behavior," paper SPE 15430 presented at the SPE 61st Annual Technical Conference and Exhibition, New Orleans, Louisiana, October 5-8, 1986

[44] Efros, D. A. : "Study of Multiphase Flow in Porous Media," Gastoptexizdat, Leningrad, 1963 (in Russian).

[45] Chaperon, I. : "Theoretical Study of Coning Toward Horizontal and Vertical Wells in Anisotropic Formations: Subcritical and Critical Rates," paper SPE 15377 presented at the SPE 61st Annual Technical Conference and Exhibition, New Orleans, Louisiana, October 5-8, 1986.

[46] Guo, B. and R. L. Lee : "An Exact Solution to Critical Oil Rate of Horizontal Wells with Water-Oil-Interface Cresting," Proceedings of the Lerkendal Petroleum Engineering Workshop, Trondheim, February 5-6 1992, 55-66.

[47] Wheatley, M. J.: "An Approximate Theory of Oil/Water Coning," SPE 14210 presented at the SPE 60th Annual Meeting, (Sept., 1985), Las Vegas.

[48] Meyer, H. L. and A. O. Garder: "Mechanics of Two Immiscible Fluids in Porous Media," J. Applied Physics, (Nov., 1954) 25 11:1400-1406.

[49] Guo, B. and R. L-H. Lee: "A Simple Approach to Optimization of Completion Interval in Oil/Water Coning Systems," SPE Reservoir Engineering, (Nov., 1993) 249-255.

[50] Papatzacos, P., Herring T. R., Martinsen R. and S. M. Skjaeveland: "Cone Breakthrough Time for Horizontal Wells," SPE Reservoir Engineering, (Aug., 1991) 311-318.

[51] Ozkan, E., Sarica, C., Haciislamoglu M., and R. Raghavan : "The Influence of Presure Drop Along the Wellbore on Horizontal Well Productivity," paper SPE 25502 presented at the 1993 SPE Meeting on Production Operations, Oklahoma City, March.

[52] Beattie, D. R. H. and P. B. Whalley : "A Simple Two-Phase Frictional Pressure Drop Calculation Method," Int. J. Multiphase Flow (1982) 8, 83-87.

[53] Barnea, D.: "A Unified Model for Predicting Flow Pattern Transitions for the Whole Range of Pipe Inclinations," Int. J. Multiphase Flow 13, 1-12, (1987).

[54] Brown, K. E.: "The Technology of Artificial Lift Methods," Petrol. Publishing Company, Tulsa, 1, 134-138, (1977).

[55] ASA Multiphase Flow Software Systems User Guide: Aziz, Spencer \& Associates Inc. (1993).

[56] Dukler, A. E., Wicks M., and R. G. Cleveland: "Frictional Pressure Drop in Two-Phase Flow: An Approach Through Similarity Analysis," AIChE. J., (January 1964) 10, 44-51. 
[57] Mandhane, J. M., Gregory G. A., and K. Aziz: "Critical Evaluation of Friction Pressure Drop Prediction Methods for Gas-Liquid Flow in Horizontal Pipes," J. Pet. Tech. (October 1977) 29, 1348-1357.

[58] Novy, R. A. : "Pressure Drops in Horizontal Wells: When Can They Be Ignored?," paper SPE 24941 presented at the 1992 SPE Fall Meeting, Washington, D.C., October. 